\title{
Comparing complementary health centres in the UK and Europe that use a combination of therapies
}

\author{
Sarah Louise Croke
}

Submitted in accordance with the requirements for the degree of

Doctor of Philosophy

The University of Leeds

School of Healthcare

December 2015 
The candidate confirms that the work submitted is his/her own and that appropriate credit has been given where reference has been made to the work of others.

This copy has been supplied on the understanding that it is copyright material and that no quotation from the thesis may be published without proper acknowledgement.

The right of Sarah Louise Croke to be identified as Author of this work has been asserted by her in accordance with the Copyright, Designs and Patents Act 1988.

(C) 2015 The University of Leeds and Sarah Louise Croke 


\section{Acknowledgements}

I wish to thank my supervisors Dr. John Lees and Professor Dawn Freshwater for supporting me through what has been a very long and difficult journey; my study intermediaries Dr Erik Baars, Dr. Chris Maratheftis, Professor Andreas Nicolaou and Jesper Odde Madsen for recruitment support and more; and the participating clinics, practitioners and patients for inviting me into their world and into their stories so I may come to know them.

I also extend great thanks to my parents, for their willingness to accompany me on the fieldwork (enabling my children to travel with me); my husband for his honesty, patience and keeping 'the home fires burning' while I was either overseas or buried deep within my thesis; my children for all their love, smiles and inspiration; and my brother, for being the only person I could 'talk academic' with, proof reading whenever asked and for really understanding me.

Finally I wish to acknowledge the support of the LCSP-ERD Trust without whose funding the fieldwork, and this study, would not have been possible. 


\section{Abstract}

\section{Comparing complementary health centres in the UK and Europe that use a combination of therapies}

Research performances in Complementary and Alternative Medicine (CAM) can often lack the holistic nature of the therapies they study, and thereby fail to capture or adequately report holistic outcomes. This creates a problem when trying to assess and utilise research results, and may even contribute to conflicting evidence of CAM potential. Current CAM literature is dominated by a variety of studies that attempt different objectives, underpinned by different understandings, but often without holistic values.

To enact a 'new quality' in CAM research this study sought to engage CAM practice, holistically, and see what can be learned from this. A novel methodology of Theatricality was developed for the task and applied, as an adjunct to Ethnography, within five clinics across four European countries (UK, Holland, Cyprus and Denmark). Findings reveal a unique view of CAM practice, wherein the target of therapy was most often people not problems, and the quality of outcomes was determined by the contextual blend of these people (characters), interventions (plot) and places (set); this was different across the different settings. The research was similarly affected, with the ability to engage more holistically where participants acted more holistically. In all cases, there was an active dichotomy between holism and reductionism, which participants both manage and mediate, in their different acts and outcomes

While the double-blind RCT remains, for many, the 'gold standard' in healthcare (including CAM) research, this study challenges those current paradigms and popular aims to enact a different story. Applying a holistic paradigm opens up a different 'intellectual space' for this, creating a new potential for both complex and complete outcomes. It also reveals important lessons about the experience of viewing and engaging with diverse social worlds (including academia), which may stimulate other researchers to seek, appreciate, value and challenge the 'quality' in their own research. 


\section{Table of Contents}

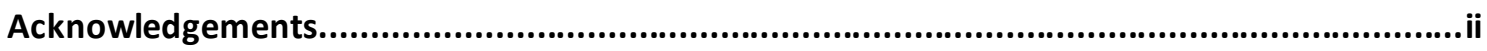

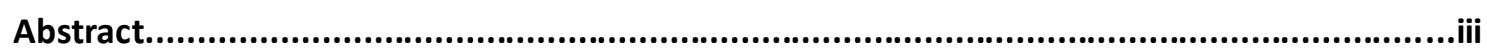

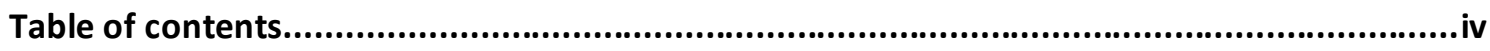

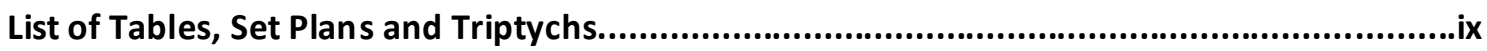

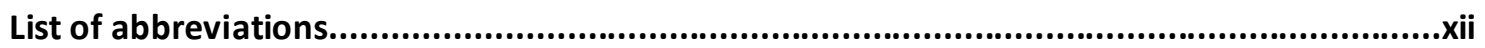

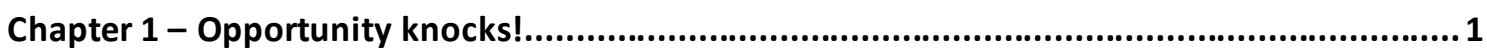

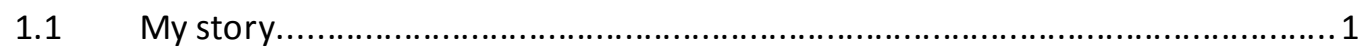

1.2 Acting as a CAM foundation.............................................................

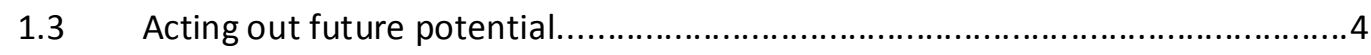

Acting with awareness.................................................................... 6

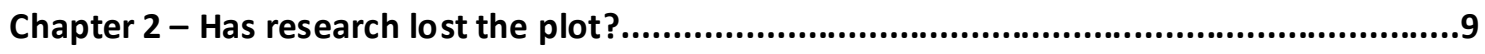

2.1 Berlin: a showcase in 'the best of' CAM? ...............................................

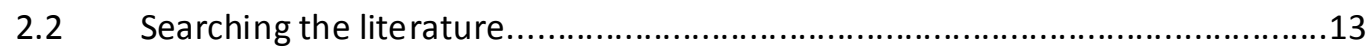

2.3 The CAM research landscape.......................................................

2.3.1 CAM clinical trials and observational studies...............................16

2.3.1.1 Trials and tribulations.............................................17

2.3.1.2 The placebo effect................................................22

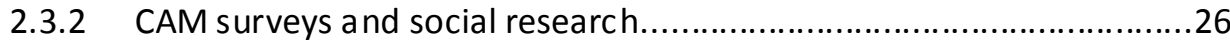

2.3.2.1 Healthcare environments............................................26

2.3.2.2 Relationships and Rapport.......................................32

2.3.3 CAM issues in research and methodological critiques......................35

2.3.3.1 The challenge of complex interventions.............................35 
2.4 Summary of the research.

2.5 Developing a question and aims for study. .40

Chapter 3 - Re-writing the script. .44

Conceiving the production..............................................................45

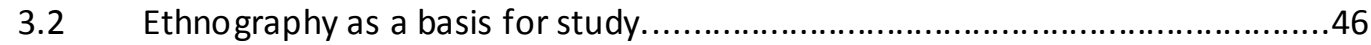

Theatre as an adjunct to ethno gra phy................................................. 47

3.4 Metaphor to methodology: the (re)birth of Theatricality?...........................48

Setting the scene for novel research............................................................50

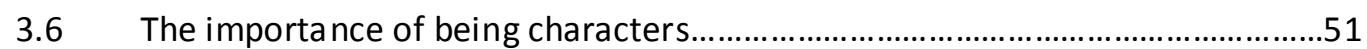

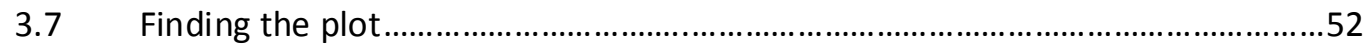

Creativity as a research process...................................................... 53

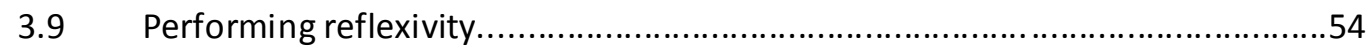

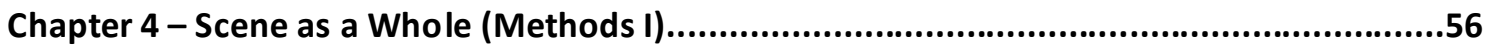

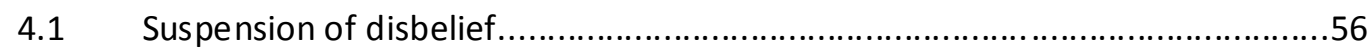

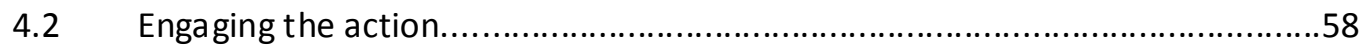

4.3 Theatricality Framework Method (TFM) .............................................60

Subjective 'spectator' observation.......................................................62

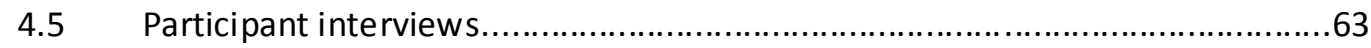

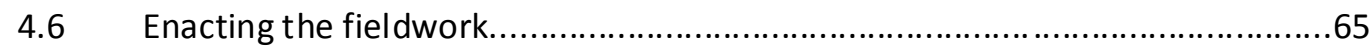

4.7 Performative criticism - a holistic metho d of data analysis.......................66

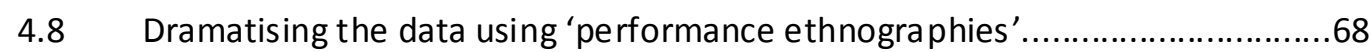

P.9 Photographic images of set designs................................................. 70 
Chapter 5 - Staging the research production (Methods II)..................................................71

5.1 Selecting and recruiting performance venues (participant clinics).................71

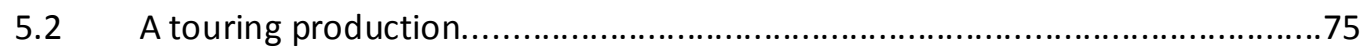

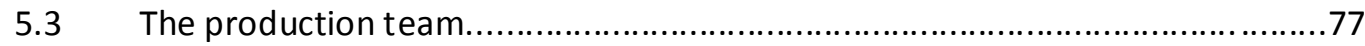

5.4 Seeking ethical and governance permissions...........................................78

$5.5 \quad$ Financial backing for the production...........................................................

5.6 Scheduling the fieldwork performances....................................................79

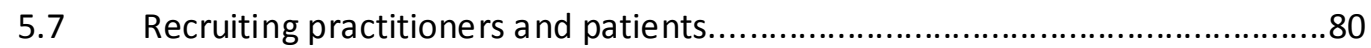

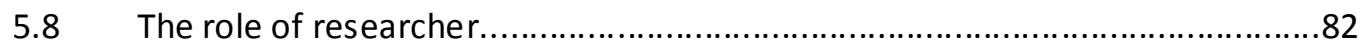

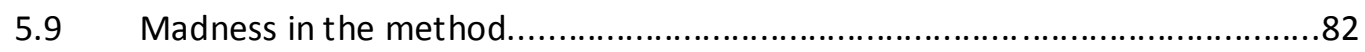

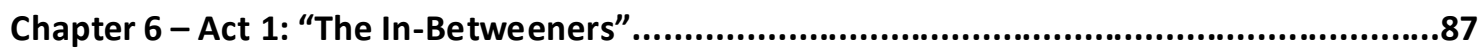

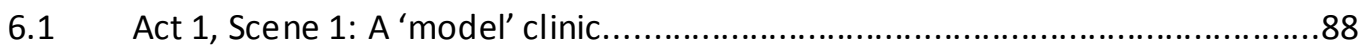

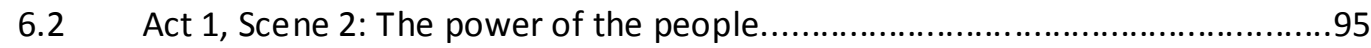

6.3 Act 1, Scene 3: The space is not eno ugh....................................................99

6.4 Act 1, Scene 4: A new breed of 'professionalism' '........................................107

6.5 Act 1, Scene 5: Critiquing the whole (holistic) thera py production.................111

6.6 Act 1, Scene 6: Reflecting on my research performance..............................115

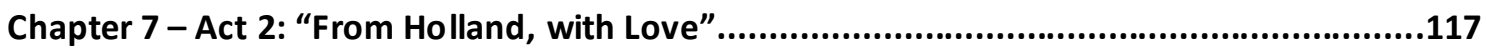

7.1 Act 2, Scene 1: Fresh flowers and sensitive souls......................................119

7.2 Act 2, Scene 2: Therapy without 'borders'..............................................126

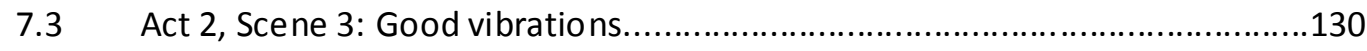

7.4 Act 2, Scene 4: Medicine in the right measure.........................................138

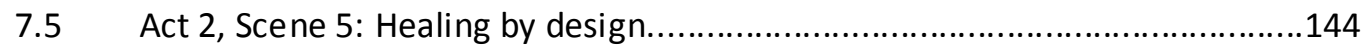


7.7 Act 2, Scene 7: Critiquing the whole (holistic) therapy production

8.1 Act 3, Scene 1: From here to eternity

8.2 Act 3, Scene 2: Me, myself and I

8.3 Act 3, Scene 3: The accidental therapist.

8.4 Act 3, Scene 4: The miracle of everything.

8.5 Act 3, Scene 5: Critiquing the whole (holistic) therapy production.

8.6 Act 3, Scene 6: Reflecting on my research performance 188

Chapter 9-Act 4: "A Tale of Two Clinics".

9.1 Act 4, Scene 1: A critical touch.

9.2 Act 4, Scene 2: The lone(ly) therapist .197

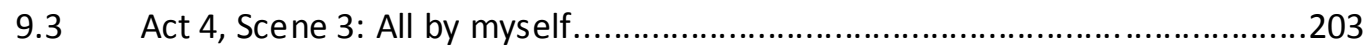

9.4 Act 4, Scene 4: Making better, making friends.....................................208

9.5 Act 4, Scene 5: Critiquing the whole (holistic) therapy production. 212

9.6 Act 4, Scene 6: Reflecting on my research performance...........................213

Chapter 10 - Finale Ultimo. 215

10.1 Reconceptualising CAM research. 215

10.2 Comparing complementary health centres in the UK and Europe. 219

10.3 Conclusion.

References. 
Appendix 2: Patient Interview Schedule.

Appendix 3: Interview data extraction sheets..................................................247

Appendix 4: Study Information Sheet (for Clin ics) ..................................................248

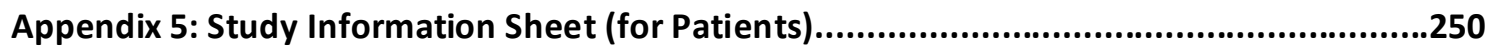

Appendix 6: Patient Participant Information Sheet..............................................252

Appendix 7: Practitioner Participant Information Sheet..........................................255

Appendix 8: Practitioner Consent Form ...............................................................258

Appendix 9: Patient Consent Form ....................................................................260 


\section{List of Tables, Set Plans and Triptychs}

Table 4.1: TFM Observation Schedule.

Set Plan 6.1: Basement

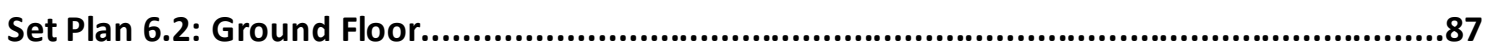

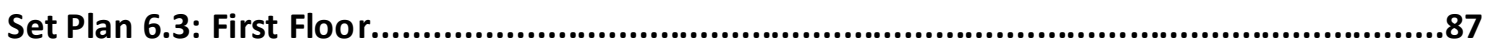

Set Plan 6.4: Attic Level..................................................................................87

Set Plan 7.1: Ground Floor Site 1...................................................................117

Set Plan 7.2: First Floor Site 1...................................................................117

Set Plan 7.3: Second Floor Site 1...................................................................118

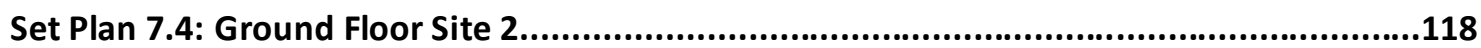

Set Plan 7.5: First Floor Site 2..........................................................................118

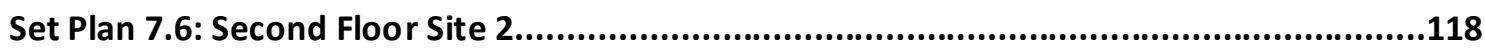

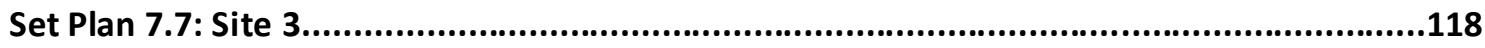

Set Plan 8.1: Ground Floor.............................................................................161

Set Plan 8.2: Basement...............................................................................161

Set Plan 8.3: Attic Level.................................................................................162

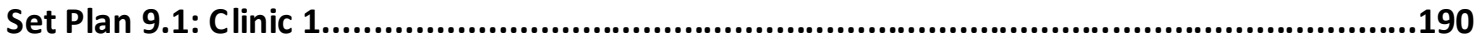

Set Plan 9.2: Clinic 2.........................................................................................190

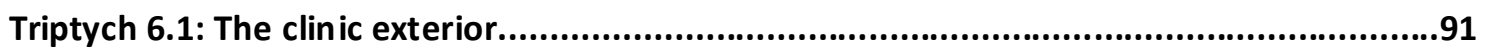

Triptych 6.2: The large reception and waiting area................................................94

Triptych 6.3: Massage and Manipulative Therapy Room 4.............................................98

Triptych 6.4: Main Office/Consultation Suite/Staff Room .........................................103

Triptych 6.5: Massage and Manipulative Therapy Room 3.......................................106

Triptych 6.6: Pilates studio (Attic) and Hypnotherapy Suite.......................................110 
Triptych 6.7: Reflexology Room.

Triptych 6.8: TCM Acupuncture Room 1.....................................................................114

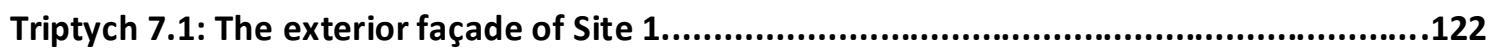

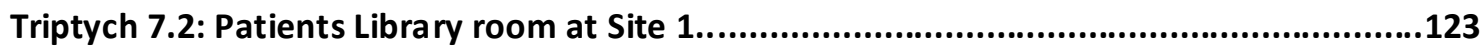

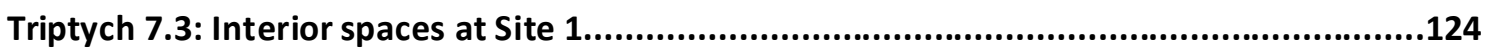

Triptych 7.4: First floor psychotherapy room, Site 1......................................................125

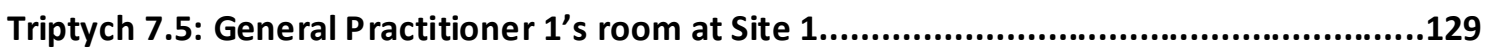

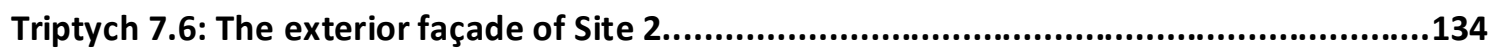

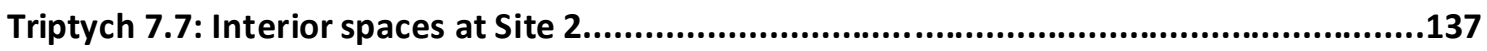

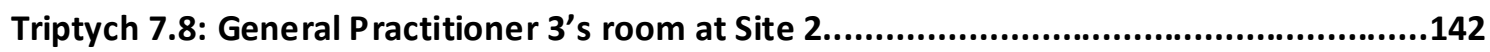

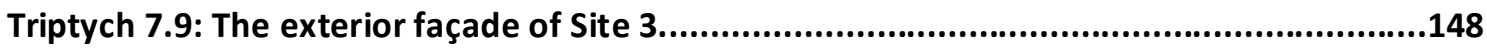

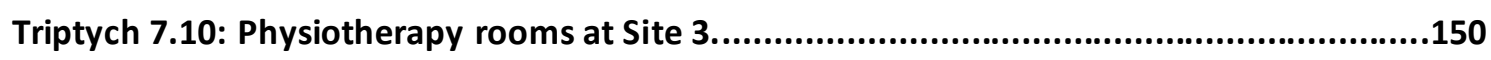

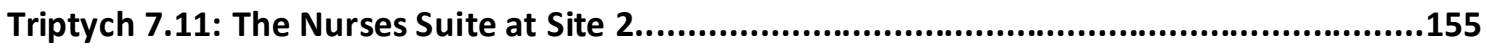

Triptych 7.12: Therapy rooms within the Nurses Suite at Site 2...................................157

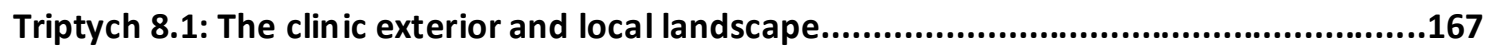

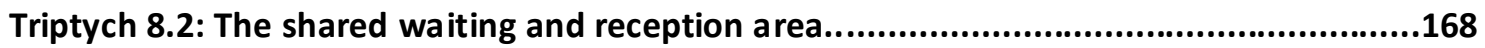

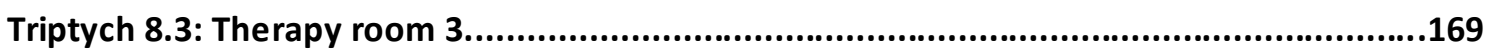

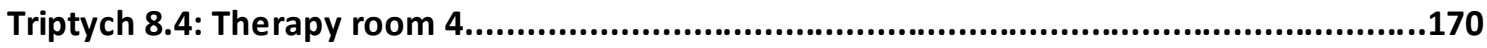

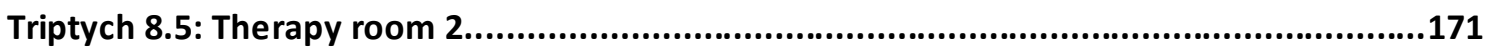

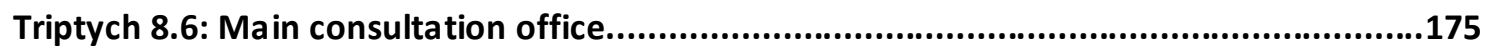

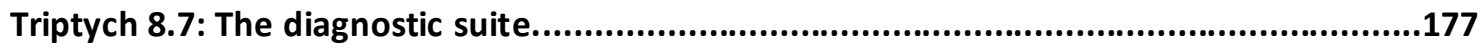

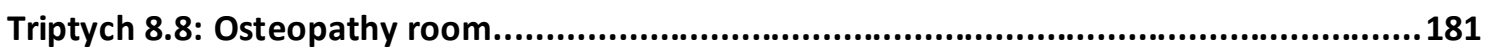

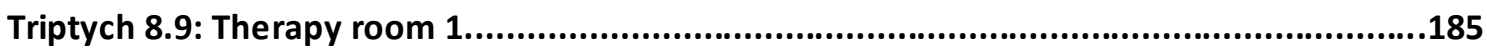


Triptych 8.10: Therapy room 6 (Bi-com). 186

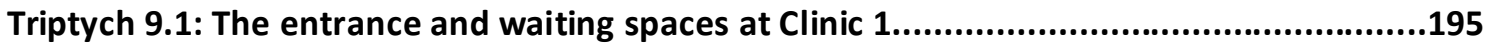

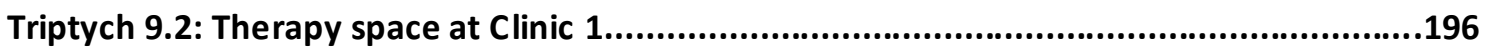

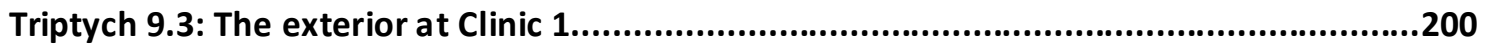

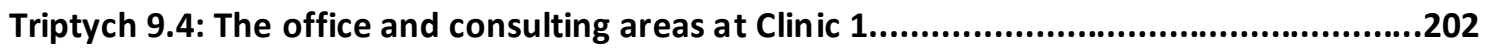

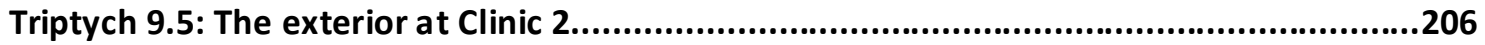

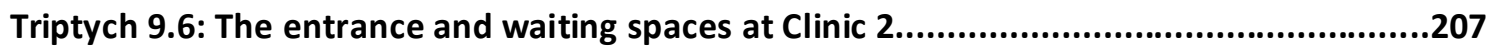

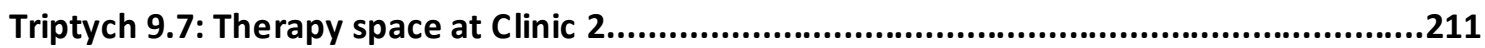




\section{List of abbreviations:}

AMED: $\quad$ Allied and Complementary Medicine Database

BI-COM: Bio-resonance Therapy

CAM: $\quad$ Complementary and Alternative Medicine

CINAHL: Cumulative Index to Nursing and Allied Health Literature

ECIM: $\quad$ European Congress for Integrative Medicine

EBM: $\quad$ Evidence Based Medicine

EMBASE: Excerpta Medica Database

GP: $\quad$ General Practitioner

IDEL: Identify and Eliminate Diagnostic Programme

MEDLINE: Medical Literature Analysis and Retrieval System Online

MRT: $\quad$ Matrix Regeneration Therapy

MWO: $\quad$ Multi Wave Oscillator

NCCAM: National Centre for Complementary and Alternative Medicine

NHS: $\quad$ National Health Service

PAPIMI: Pulsed Electromagnetic Field Generator

PSYCINFO: Psychological Information Database

RCT: Randomised Control Trial

RSS: $\quad$ Rich Site Summary

TCM: $\quad$ Traditional Chinese Medicine

TFM: $\quad$ Theatricality Framework Method

WHO: World Health Organisation

WSR: Whole Systems Research 


\section{Chapter 1 - Opportunity knocks!}

Every room is a stage, every public space is a theatre, and every façade is a backdrop. Each has places for entry and exit, scenery and props, and a design that sets up potential relationships between people (Read, 2005:53).

Like the therapeutic world with which it engages, this research production has much complexity. It is a story of my journey to becoming both broken and whole again as I set about redefining the ways in which research can engage with CAM therapies - and I (as a CAM practitioner) can engage with research.

The opening quote from Read (2005) sets out what I believe is a missing dimension within CAM therapy research: an understanding of how different contexts of therapy may create different dynamic and environmental perceptions, participant, affection and outcomes.

I want CAM practice to be judged fairly and, for this to happen, CAM research needs to better reflect the full nature of therapies including their heterogeneity and complexity. Through this written performance I seek to connect with the realities of these wondrous worlds and those who live there, bringing back a unique story of CAM to share with the reader. We may both be transformed as a result.

\section{$1.1 \quad$ My story}

I first encountered CAM over 20 years ago when I used, and then trained in, Remedial Massage Therapy. More complementary than alternative at the time, my early work was delivered primarily as a physical therapy for physical injuries, located outside NHS funding and guidelines yet supporting the medical model of healthcare. In my early days as a practitioner, I admit having limited awareness of any other CAM modalities and no awareness of vitalistic philosophy as I now understand it to be, taking the view that if it didn't make sense to 'science', it couldn't work. I had trained in bodywork instead of conventional physiotherapy, primarily due to unsatisfactory outcomes with the latter as a teenager, in a training that was designed and delivered to complement mainstream healthcare. The institute where I trained had been producing therapists for this purpose since the early 1900 's, when massage was an accepted part of mainstream healthcare, often delivered by doctors and nurses (Goldstone, 2000), and held an envious reputation for the quality of therapy and results its practitioners delivered. I see now that this doctrine was so powerful it effectively kept me away from exploring any further - until I entered University to further a 'complementary' clinical career. 
While undertaking a combined BSc (Hons) Complementary Medicine \& Health Sciences, I had my dominant discourse challenged and my life changed forever. Stimulated not as Ernst (2010a) argues, because of the media, friends or past unsatisfactory therapeutic relationships in orthodoxy making me become critical of biomedicine but rather the 'alternative' view providing answers where previously I had only questions, solutions where there had seemed no hope and a solid theoretical philosophy for explaining health and illness that made sense, I chose to make the leap from adjunctive to alternative! My world was now upside-down and everything I had ever been taught or believed was suddenly ina dequate.

I was facing the very real prospect of only ever providing clinical therapy with a palliative or limited intent, until I found a way to be a different practitioner. I re-positioned myself as an interim or gateway into holistic medicine, connecting the different person I was now (living, teaching and perceiving health and life with vitalistic principles) to both the world around me and the world I wanted to live in. Adopting a new comprehension, I became naturally more 'therapeutic' for my patients, able to help interpret their wider issues or direct their gaze (when appropriate) beyond biomedical conceptions of health and disease and towards therapies or interventions they may have had little awareness of to consider before. As the cornerstone of all my participation (health and social) I felt like I was now working 'with the light on'!

Seeing CAMs differently led me to also see research differently. Gaboury et al (2012) have noted that many practitioners can be limited in their own knowledge, experiences and perceptions of CAMs, which can in turn impact their ability to practice 'evidence-based' medicine; and I would agree this once described me. Able now to view an 'inadequacy' in studies that try to fit holistic practices into reductionist research models, I had a desire to reposition myself again as a practitioner-researcher; and this has become my opportunity.

\subsection{Acting as a CAM foundation}

It is a foundation of holistic philosophy that the whole is greater than the sum of parts (Paterson \& Britten, 2008) meaning that in health or healing it is the cumulative effects of all things (not just theory and practice) that creates the potential for either wellness or illness to occur. This is something that I have borne witness to, many times during both my personal history and my clinical career.

If I look back now, I can see how this theory explains every illness episode I have been subject to (either myself or someone close to me) and equally how, indeed if, any recovery has 
occurred. Continuing to the present day, I have been battling with a series of emergency health scares relating to my youngest child, throughout the entire time I was completing this thesis. My primary supervisor once said to me that only when I completed my thesis would she become well. This may have been true but I needed to right conditions to support me first, and this was difficult to create, for a long time. The change came from realising that, contrary to CAM philosophy, I had become increasingly isolated (a most unhealthy state) and this had created the potential for illness.

This is something I have experienced before when those I have placed trust in have let me down, making me rely only on myself. Here, I had competing forces of family, academia and discourse with no sign of balance between them and me 'caught in the crossfire'. Feeling 'under attack', the conflict I had entered sent me further into the margins where my confidence sank to such a low ebb that I almost gave up, convinced that no one would want to listen to what I had to say anyway. Finding how to reconnect with myself and fight my way back has been perhaps one of my greatest challenges throughout. Through making connections, however, I found the potential to foster support, begin to heal and thereby complete.

In terms of connecting this to the issue of CAM research, everything seems to just 'work better' when it is connected (I refer to myself and general CAM theory also), and yet CAM research does not appear to design trials or studies that consider this principal. Why this should be I am not sure, except perhaps the dominance of mainstream medical discourse, which favours separation over connection, has such a strangle-hold on researchers they feel unable to 'act' better? Alternative philoso phy had already broken my cycle, so I wondered if a similar approach may help here (much like when a patient who, after unsuccessful treatment at the hands of a conventional practitioner, turns to alternative approaches seeking a more holistic solution).

As others before me have sought (unsuccessfully) to 'blend' paradigms, I felt it may be a productive turn to look beyond healthcare to another possibility, where the concept of a 'totality' and cumulative effects also exists. One such possibility is theatre. Here, just like in therapy, "everything that takes place in this specially constructed alternative world is a vital part of the whole event" (Duggan \& Grainger, 1997) and it may even be argued that theatre's holistic nature provides a natural parallel to CAM practice. 
This is something I have always been aware of, although perhaps more implicitly, than explicitly. I was a theatre practitioner (trained but then amateur) before beginning my clinical CAM career and have therefore always (where parameters allowed) staged my workspace with intentional design much like I would stage a theatre set! For example, I always made small additions to enhance the space (lighting, candles, music) however limited or flexible my control or, when I had more freedom, my position and arrangement of the physical parameters were adapted to suit my use best. In either, being a private, independent therapist meant I could often pragmatically make the best of wherever I found myself. I intuitively know how my own experiences in different therapy 'staging' (patients/clients homes, a private gymnasium, Turkish baths, a primary care facility or my own commercial let) are relatable to the quality of care I deliver and outcomes observed there, finding each one to create the parameters for action and expectation. A phenomenon previously explored by Burns (1972), this seemed to me to be a natural foundation for approaching the capture and appraisal of CAM complexity.

Of course, when Read (2005) wrote "every room is a stage" he was not talking about CAM therapy; rather he was talking about architecture and how theatre, as a 'sister' art, may provide a novel way of understanding and engaging with human interactions and spatial relationships. As applicable to shopping malls as to open spaces, Read's argument was that viewing space not as an art of composition or engineering but as a 'performing art', creates the possibility of reflecting "real situations, characters, and places...in real time, at a real scale, and with real people" (Read, 2005:53). I liked this.

This perspective is based on an approach first trialled in 1919 by Edward Autant (an architect playwright) and is one that would traditionally fall outside of the gaze of many healthcare researchers, but I feel could play a significant role to raising engagement and understanding with 'alternative' social worlds, such as in CAM. This view of 'place' in shaping situations (Read, 2005) is one that I easily identify with, having also spent much of my life involved in amateur theatre. In the spirit of adventure, the only way to find out would be to try it.

\subsection{Acting out future potential}

I am a real CAM practitioner and I care passionately about CAM. I engage it as a primary health approach for myself and my children and feel others should also have the opport unity to benefit as we have. This is why I want 'good quality' information to be shared about the potential benefits and limitations of each approach, so people can make informed judgments on how they want to access their healthcare, and policy makers my seek to provide this. 
Unfortunately, this 'good quality' information seems slow to appear and this is a potential limit on the availability and accessibility of CAMs. In choosing to enter the arena myself I aim to see if I can 'do things any better', or at least 'add something' to the debate.

I remember the first day I walked into the School of Healthcare. I was full of bullish excitement and enthusiasm, happy to be making this thriving research department, with a culture seemingly supportive of CAM research (hosting five other CAM oriented post-graduates) and many high profile healthcare researchers resident, my 'home' for the next few years. As a scholarship student the title for my study had already been selected for me, however beyond this, I had total control of a very broad remit indeed and I quickly threw myself into everything that was on offer (almost scared to miss out on even small opportunities). I was a regular attendee at local CAM research groups (CAMFRY and ACHRN) and national/international conferences (ECIM, IN-CAM and CAMSTRAND) where I met many interesting characters, including practitioners from a variety of disciplines and respected researchers and professors (some of whom I had been 'reading' since I was an undergra duate), learning from them what I thought it 'took' to become a successful researcher. A positive act, this served nicely as a direct source to all the latest information and developments in CAM research, which became a critical guide to both my personal and professional development.

My appointed supervisors, neither of whom were 'strictly CAM', fast became respected friends and I loved to have philosophical discussions with them, in the early days (although I'm not sure either of us were aware of the journey we would eventually be making together). They had each arrived with an interest in CAM methods via their primary fields of nursing, counselling and psychotherapy, writing on these within the general healthcare literature.

Energised, I think, by my initial passion and commitment they gave me more freedom than I really knew what to do with; always afterwards challenging my choices so I would eventually become an independent thinking researcher (capable of initiating my own programme of study). I felt supported by my supervisors and the various participants, who seemed bemused almost that this hadn't happened before, which raised my confidence higher.

But like a flare that eventually 'crashes and burns', I found once I was on my own the reality of my task started to worry me and, as my early attempts at writing fell wide of the mark, I started to ask myself if I had taken on too much? But then there were so many people depending on me, I couldn't fail now! 
I think I actually became blind about how to achieve this, and as my inability to complete the task set stretched longer and longer I began to experience intense periods of illness and (I suppose) depression. My supervisors too became openly frustrated with me (one time, during supervision, my primary supervisor hit the desk so hard with a draft I had sent him I can still feel the shockwaves); they still saw my potential, but I just couldn't achieve it.

My first submission of this thesis proved clear evidence of this. I was referred for further work (having given at least a satisfactory oral performance). My supervisors still seemed committed to supporting me, but I remained blind to my eventual way forwards, chasing shadows and wrong turns instead. In the antithesis of CAM I was looking outside myself for answers - not inside (where true healing comes from). This was when my youngest child became ill, diverting my attention away from the 'impossible task'! I tried to continue, but the strain became too much and I eventually I took advice and permission to suspend my studies, to try and recover sufficiently to reapply myself again.

Little positive happened during this time, and I came back no clearer about the solution, which continued to show in my work. The timely reading of another thesis brought the turning point I needed. Hoover (2012) 'spoke' to me and opened my inner space, previously tightly closed, to the weakness I had long held within, that would eventually make me stronger (although I always feared that to expose this would mean ultimate disaster!). This was a different kind of stronger than I had been before; a more considered stronger. This was an unforeseen potential for me. I thought, until then, that my primary goal was to develop CAM (and reform) research - I never considered that I needed 'reform' too.

\subsection{Acting with awareness}

Wardle (2008) and Lewith (2008) have previously argued that CAM practitioners should be encouraged to develop research in CAM, as their intimate knowledge of modalities can be of great benefit. Perhaps unaware of the full nature of this challenge, I relished the opportunity to directly impact the practice and outcomes of research in this field and, in line with this advice, have sought to maintain a level of active clinical practice throughout, which has indeed provided benefits; such as helping me to gain access to clinical settings, appreciate the complexities involved in their practices and outcomes; and (by engaging my underpinning knowledge of broad and diverse guiding theories/philosophical traditions) also recognise when these are either contradicted or absent from trial design and reporting. 
De-mystifying CAM is a vitally important task. It provides the foundation for all succeeding work influencing how particular modes of therapy may be either dismissed or accepted. Too often, however, this appears based on limited knowledge or appreciation of the underpinning principles or contextual impact. I have found, many times, when patients arrive at my therapy couch claiming to have previously tried acupuncture, for example, within a physiotherapy department in an NHS hospital (applied within the 'medical model') they also have acquired a perception of Traditional Chinese Medicine (TCM) based on this single distorted exposure to 'acupuncture'. Bearing limited (or no) relation to the whole system of Traditional Chinese Medicine, absent or the original principles or context, the potential for success may be markedly reduced - but this is neither noted or acknowledged, with other alternative modalities also being similarly judged, just by association.

This is clearly unsatisfactory, as acting without awareness of the potential differences within these complex approaches means patients may discount a particular therapeutic modality as being of no benefit for their particular condition (best case scenario), or of no benefit generally (worst case scenario), when a different therapist or context could yield a very different response. I personally doubt anyone who has had the fortune to encounter and compare NHS delivery with private TCM Acupuncture (or any other variation) could not identify key differences between these applications, experiences and outcomes.

The idea that different performances of therapy create different experiences for patients is not new. It has been noted by Carter (2003) that provision within the private sector is very diverse and for many reasons (i.e. training routes, occupational standards, resources, authority and aims of the practitioners etc). Moreover, heterogeneity of practitioners and practices is a confounding variable that many CAM researchers can stumble over during clinical trials (Ritenbaugh et al, 2010). Better done in mainstream healthcare, the impact of hospital design on healthcare outcomes is well studied and new hospitals often adopt 'evidence-based design' in their construction (Shur Bilchik, 2002; Sloan Devlin \& Arneill, 2003). Similarly, physicians and practitioners across many disciplines know they can engender positive attitudes or compliance in patients from developing good therapeutic relationships in the ways they communicate with them (Busato \& Kunzi, 2010).

I am concerned the drive to bring these studies into line with other allied approaches (pursuing the Evidence Based Medicine agenda and/or efficacy beyond placebo), and the current focus on issues of safety and cost effectiveness, may be drawing CAM researchers away from the task of fully exposing and exploring the whole, complex and unique natures of these therapies 
before we have fully learned to understand them. This seems, at best, premature to me and may even be a maintaining factor in the inability of many CAM studies to meet the required methodological standards for quality and rigour. Until CAM research takes a broader, more holistic view of CAM interventions, the nature and potential of these therapies may remain poorly understood and the evidence for their use effectiveness elusive. I expect there are many current CAM research practitioners who feel their approaches are already holistic, but through my own 'agitation' (a homoeopathic term) I have found that even I am not always as holistic as I think! Re-search is, after all, about looking again.

Realising this vision (or at least attempting to) is the story of my thesis. With thought processes, actions and learning all contained within, the reader may connect with the harsh lessons this has taught me, as I have learned both about myself and the topic. Like therapy, my observations and performance is also a dramatisation of the context in which I have performed it.

The 'paradoxical theory of change' argues that change can only happen when we become what we truly are, not when we are trying to be something that we are not (Stevenson, 2010). In the spirit of reform, I therefore have tried to embody who and what I am in this enactment. I feel that CAM research has within it, through engaging holistic philosophy, the means to actively expose and explore complex issues of context within performance, appraisal and design in a way that is different; unhindered by reductionist discourse and with benefit to many working across CAM healthcare and beyond. Having developed such awareness, over many years, I feel no that the time has come to do something about it. 


\section{Chapter 2 - Has research lost the plot?}

Complementary and Alternative Medicine (CAM) is a social phenomenon. Surveys from several European countries suggest increasing use over the last decades with up to $70 \%$ of citizens now having used some form of CAM (Nissen et al, 2012) while the most recent UK data for CAM use suggests a prevalence of $26.3 \%$ (Harris et al 2012). Still, the evidence base for CAM thera pies remains lacking in all measures.

I have personally been engaged with CAM therapies for over 20 years, first as a patient, then a thera pist, later a CAM undergraduate, an FE/HE lecturer and finally a PhD researcher. As I look back, I am saddened at the limited progress over this time, compared to my expectations when I started out, with regard to patient accessibility and engagement with CAMs. Then, therapies seemed to offer the perfect solution to everything that was lacking within mainstream healthcare and a new frontier of 'integrated' medicine beckoned where people could choose between or combine conventional medicines with natural remedies and alternate treatments, if biomedical approaches alone didn't seem to work. More research was just needed first!

Despite the best efforts of many, the reality remains that quality CAM research has been slow to arrive. This is not to deny all the unquestionable good that has come from the efforts of researchers in this field over the last $20+$ years, particularly in terms of the global picture and trends of prevalence and modes of CAM use, attitudes of both professionals and the public in regard to CAM, and more recently an interest in economic and safety issues. This provides a foundation for all that has yet to come and, from appraising the success and struggles within this body of knowledge, I am sure new directions will emerge. However, divisions between conventional and alternative healthcare have persisted and, in many areas, CAMs largely remain on the 'fringe' of healthcare: so there is more work to do. I believe there is currently a new imperative for CAM research to step up and produce the right types and quality of evidence to show benefit, where it lies, as biomedicine struggles to keep pace with chronic illness, antibiotic resistant infections and the rising costs of technology and drugs to treat them. The question seems to me then not 'where do we go next' but 'how do we go next'?

\subsection{Berlin: a showcase in 'the best of' CAM?}

A solid platform was needed for this, so just 6 weeks into my journey, I travelled to Berlin to attend the $2^{\text {nd }}$ European Congress for Integrative Medicine. This 3 day conference was intended to be "an innovative platform for medical practitioners and healthcare professionals 
as well as scientists, health politicians and sponsors" to present and discuss "Integrative Medicine [which] combines conventional Western Medicine with complementary methods of treatment such as naturopathy, homoeopathy, and acupuncture" (ECIM, 2009:4). I felt this would be a great place to start from, given the breadth and freedom of my remit.

I had such enthusiasm when I arrived, eager to see and connect with the current and latest 'innovations' in my field. One of the first symposium sessions I attended was entitled "Complementary or Integrated? - Clarifying the concepts". Harald Walach and Claudia Witt, two of the leading CAM professors in Europe, were the Panellists discussing different opinions, interpretations and implications of CAM terminology, with the audience. A diverse range of clinicians and researchers were also present so I was hopeful of a good and varied discussion.

The session had one dominant theme: the use of CAM as an umbrella term for this mode of medicine and the clear dissatisfaction this holds for many. Soon participants became obsessed with trying to agree a 'new' umbrella term, finding this current one lacking or misleading for their specific use, with complementary and integrated medicine (CIM instead of CAM), complementary and integrated healthcare (CIH instead of CAM), plurality in medicine (PIM instead of CAM) and patient-centred care (PCM instead of CAM) all in the running. Harald Walach suggested that "CAM is best as it prevents Medicine colonising over CAM, as in Integrative" but this didn't appease many of those in the room (a lot of physicians) who seemed to find the ' $A$ ' for 'alternative' in CAM somehow threatening, and that distancing themselves from this may benefit their therapies in becoming more accepted by mainstream medicine? Harald followed up by stating, "If you integrate, where are the alternatives?", so no resolution came.

I was interested in how the competing concepts, rather than the language, of conventional and complementary medicine may be integrated and I asked the panel this directly.

\section{I began:}

S. Croke We may see how on a practical level we could integrate methods, but how do you each see the integration of ideologies?"

I wanted to highlight how they saw the integration of theories working in a clinical practice setting.

\section{Claudia Witt was first to respond:}

C. Witt

That is a whole-other question! 
Harald Walach swiftly followed with:

H. Walach

I agree with Claudia

And neither of them was willing to be drawn on it further. I found this whole episode quite frustrating although it did spark some suggestions for me about issues in CAM research.

I reflected on this later that evening and noted in my diary:

"This highlights to me that the only people to whom this debate probably even matters are the academics and policy makers who feel they need to have clarity in order to have dialogue - but the lack of any clarity does not seem to hold back patients IN THEIR EFFORTS TO PRACTICE INTEGRATIVE MEDICINE IN A WAY THAT WORKS FOR THEM" (capitals added).

When I discussed this episode with my primary supervisor, we agreed that it reminded us of the 'Mad Hatter's Tea Party' in Alice in Wonderland: going round and round but never forwards. We began to surmise whether such inconclusive discussions may still be endlessly continuing and what, if anything, may interrupt this. This discussion too had the potential also to go round and round but never forwards!

It was almost a theme of this whole event for different speakers to side-step the issue of theory and philosophy, particularly when 'relating' CAM findings to Western understandings of evidence. Perhaps this is what they meant in the programme, when they spoke of "crossing the ideological borders between these systems" (ECIM, 2009:4)? I found this was highlighted best in a plenary session showcasing the leading CAM institutes in Europe, where I grabbed the opportunity to question Dr Peter Fisher from the recently renamed 'Royal London Hospital for Integrated Medicine' (formerly the Royal London Homoeopathic Hospital) about his understanding of CAM, in relation to integrated medicine.

He began:

P. Fisher

The new name reflects what we do. Combining the best of complementary medicine with conventional medicine.

So I asked:

S. Croke

You have used this term twice in your presentation, and others at this event have used it too, but what do you consider to be the best of complementary medicine? 
P. Fisher

Oh...erm...well, I suppose I mean good standards, properly organised, regulated, trained, run etc.

Dr. Fisher was one of many here to use the term 'the best of CAM' without clear definition, however the manner of his response made it clear that he hadn't actually thought about this before using it! He approached me later that evening, at the gala dinner, and personally commended me for asking my question. It was nice to feel that I had been 'heard' by him, although he still could offer no better response than the 'off-the-cuff' remark he had made earlier. This made me wonder whether this term is currently limited to 'political rhetoric' and if CAM research should therefore be first seeking clarity about the different CAM practices and dissecting what CAM means before it rushes towards 'integration'? I began to sense that unless or until this is done, the direction being forged by current research leaders (whereby CAMs seek incorporation within 'future medicine' through conforming to the biomedical paradigm for both clinical trial evaluations of therapies and explaining mechanisms of action) may never result in any satisfactory outcomes. These 'stars' of the circuit were fast in danger of becoming 'false gods' to this novice, yet eager, CAM researcher (me)!

Other discussions I held with delegates and presenters seemed to confirm the situation that holistic principles typically suffer when conducting (credible) CAM research. I found it hard to accept that this 'norm' has to be conformed with, although among these 'demi-gods' and their followers I found few examples to challenge this. Ritenbaugh et al (2010) has previously noted that, for CAM researchers who have moved over to CAM after having first developed a career within more mainstream discourse, the challenge of familiarity and expertise with a reductionist paradigm can be difficult to move beyond. This led me to question whether those CAM researchers I had observed lacked either the freedom; the ability; or maybe even the desire to develop and improve their work? I seemed to witness elements of that here.

This was a key moment in my development. The Congress showed me where CAM research was currently engaged and the challenges I would face if I wanted to become part of this community. I left feeling, no screaming, that the gaps between current research practice (typically reductionist paradigm) and therapy practice (typically holistic paradigm) do not appear to be actually 'crossed' at all and this needs to be addressed, urgently. I knew then that I wanted to contribute to this! I also learned that I must not compromise on my philosophical beliefs if I am to serve this goal. 
As a whole experience, this event was major catalyst for me revealing clear gaps in current directions of CAM research, on a European level, and affording the opportunity to begin making the kind of connections that would later become integral to the performance of my whole study. Still, this event saw such impressive delivery of keynotes and proposals that many attendees found it easy to accept the ideas presented, even becoming compelled to contribute to them.

\subsection{Searching the literature}

I returned home, after this 'live' engagement to immerse myself in the broader CAM and healthcare literature. As I was only just beginning to see where I wanted my research to take me, I decided my engagement with the literature at this stage should provide a "scaffolding" (Thorne, 2008:53) to support any eventual direction, knowing that where I travelled would have come from within the data, rather than outside (much like true healing comes from within, rather than outside). This does not mean I claim the capacity to 'heal' CAM research, but with no clear brief already in place this was an authentic approach for me to take.

Engaging as a practitioner first and researcher second, I quickly learned that CAM has seen many areas of inquiry over the last $20+$ years, increasing both in volume and quality over this time: from attitude and prevalence studies to RCT and pragmatic trials. Reflecting the breadth of thought and presence of complexity within these different modalities, my initial searching was similarly broad as I began to engage the amount of heterogeneity, not in CAM practice per se (as I already had this) but in CAM language and meaning. I began first looking for anything holistic, to discover if the largely 'reductionist turn' I had observed in Berlin was merely a current trend or an institutionalised convention.

I carefully constructed a list of search terms that I felt may be a catch-all for the different dimensions of therapy practice (actor*; acupunctur*; alternative; ambien*; arena; aromatherap*; atmosph*; ayurveda; cam; Chinese; chiropract*; clinic; complementary; complementary medicine; drama*; effect; environment*; Europe*; experience*; folk; healing; health*; herb*; holistic; homeopath*; homoeopath*; impact; indigenous; integrat*; massage; medicine; meditat*; music; natural; osteopath*; outcome place; placebo; practice; practitioner*; psychotherap*; qi; quantum; reflexolog*; reiki; room; scene; set; setting; space; spiritual; stage; staging; tcam; tcm; theat*'; therap*; touch; traditional) which I entered, in various combinations, within prescribed search engines (AMED; EMBASE; PSYCHINFO; OVID Nursing; MEDLINE; and GLOBAL HEALTH). This produced a total hit of 150676 articles, of 
which 2-40 studies showed potential relevance. Further inspection of these revealed that nowhere had a holistic approach (at least within my understanding of the term) been applied.

Deviating this strategy to ensure I did not overlook anything, I also added a more organic and non-linear method to this process: hand searching of CAM specific journals (BMC Complementary and Alternative Medicine, Chinese Journal of Integrative Medicine, Complementary Health Practice Review, Complementary Therapies in Clinical Practice, Complementary Therapies in Medicine, European Journal of Integrative Medicine, Evidencebased Complementary and Alternative Medicine, Forschende Komplementärmedizin / Research in Complementary Medicine, Integrative Cancer Therapies, Integrative Medicine Insights, Journal of Complementary and Integrative Medicine, The Journal of Alternative and Complementary Medicine); setting RSS updates to alert to all new publications; and reference chaining into various texts and publications often beyond CAM and even broader healthcare.

Despite its lack of technological wizardry this proved a successful strategy for identifying relevant material, alongside expanding and refining my search. I was creating a climate where

"new discoveries were made, tangentially related" to my quest (Thorne, 2008:57), which also put my experiences at the 2009 Euro pean Congress of Integrative Medicine into context.

Running coincidentally alongside my study has been one of the largest scale CAM research studies ever funded. With wide reaching implications, CAMbrella (see www.CAMbrella.eu), has been a $€ 1.5$ million Euro-mapping exercise of the CAM landscape within Europe, including participants from 16 institutions across 12 countries, actively participating in a number of projects and focussed on the topics of CAM terminology, attitudes and needs of citizens in relation to CAM, prevalence of CAM therapies, legal status and regulation of practitioners and future research directions (Eardley et al, 2012; Fischer et al, 2012; Nissen et al, 2012; Von Ammon et al, 2012; Walach, 2012). Such has been the expectation of this new body of work that the authors claim they will become canonical texts for years to come (Walach, 2012) and although results were published in November 2012, too late to inform or influence my study design and execution (a full year after I completed data collection), I have been able to consider their findings alongside my own and, indeed, it is notable that some aspects of their findings display congruence with mine. However groundbreaking the potential for this or any other study, it remains that even this literature continues to overlook the fundamental essence of many CAM approaches. 


\subsection{The CAM research landscape}

Something I was well aware of, before I started, is that underpinning the landscape of research in CAM is a unique and overwhelming heterogeneity. Delivered under the umbrella term of CAM (complementary and alternative medicine) common definitions include "a broad set of health care practices that are not part of the country's own tradition and are not integrated into the dominant health care system" (WHO, 2000:1) or "non-mainstream practice" which can be used either "together with" or "in place of conventional medicine" (NCCIH, 2015:1); also subject to local definitions, and who is doing the defining (Kelner and Wellman, 2003), inclusions and exclusions within these can vary across cultures and countries (Dobos, 2009).

I had a very real understanding of both the range of potential modalities for patients to access and the different theories or practices that underpin them after my earlier undergraduate education in CAM: yet found this remained a challenging environment to engage with. Perhaps my first observation is how inadequate this definition is, particularly for research purposes. Defining something by what it is not, rather than what it is, is a further layer of confusion in an already impossible task, at least for anyone intent on serious immersion in all the potentially relevant literature. As my remit for this study included comparing centres that offered a combination of therapies, and at this stage I had yet to identify any centres or therapies to compare, this represented an inordinate task (despite the advantage of my prior situation).

I am not the only one to find this problematic. Hoping to harmonise research in this area, the Cochrane Collaboration have taken up a new operational definition of CAM put forward by Wieland et al (2011) that effectively consists of a closed list of therapies and products deemed relevant to a 'standardised classification' of CAM, but with the potential to be expanded over time. It is too early to say if there is the potential within this model to retain the richness and diverse nature of lived experiences of CAM while still 'fitting' standard classifications, or whether this will prove the desired aid to systematic reviews.

The CAMbrella group have also sought to improve on the single, universal definition accepted by many as currently the best option available (Gaboury et al, 2012). Their response after consultation, however, is that a universal definition reflecting the vast diversity of systems, disciplines, procedures, methods and therapies available is not fruitful, and suggest stakeholders instead specify their own definition in relation to their specific project; despite the negative implications for research and clinical practice (Falkenberg et al, 2012). Not the 
resolution initially sought, this rejection of a homogenous term for a heterogeneous practice as 'unsatisfactory' is so mething I concur with.

But this 'new' clarity was not in evidence when I began my study, yet I needed to determine where to apply myself, so I set about exploring the literature; first with the aim of first seeing whether the trend towards reductionist methods showcased in Berlin was a wider phenomenon, then to uncover the possible reasons for following this and finally the possible implications for studies and findings. As a broad sample of the CAM literature, these offer insight and illuminate omissions that together, may suggest a positive application for my study. For the sake of navigation, and to find some clear direction, I reviewed this eclectic and heterogeneous body of knowledge under 3 distinct but related headings, with various subheading s. Together, these create an interconnected whole - to serve me best in the aftermath. These are: clinical trials and observational studies; surveys and social research; and issues in research and methodological critiques.

\subsubsection{CAM clinical trials and observational studies}

The field of CAM research has become more established in recent years benefitting from investment in selected Universities and specialist CAM research centres. Researchers from across the globe now have increasing opportunity to share their work and develop collaborations in a number of respected CAM specific research conferences, meaning the quality and volume of this body of work is growing and the Cochrane Collaboration can now produce regular review papers on the topic. Yet there remains much to learn about CAMs.

Within the context of research, EBM (evidence based medicine) provides much of the underpinning exhibited in current practise. Proposed within CAM therapies, and other nonpharmacological modalities (physiotherapy, psychotherapy and nursing) as the best way to create evidence for what they do, removed from the burden of proving theory and philosophy (often incompatible with the scientific model), there has been much activity within these over recent years with randomised trials (the gold standard) being accompanied by pragmatic ones, as development in methods seeks to increase the validity and transferability of outcomes. However, as Greenhalgh (2012) notes, there can be unintended consequences in disconnecting theory from practise and distorting modalities beyond natural recognition, as this may equally lead to less (rather than more) valid studies and outcomes. While I applaud that efforts are being made to improve the evidence for CAM therapies, I agree with Greenhalgh (2012) and am concerned current approaches may be sacrificing so much to appease so few. 


\subsubsection{Trials and tribulations}

In their study of Chronic Pain, Ostermann et al (2008), provide a clear example that carries typical factors present (or absent) in many studies. They treated 100 patients with chronic pain three times over 24 days by applying a rhythmic embrocation with Solum Oil (a lavender/peat extract). Key findings were significant benefits in improving mood (mean scores of Zerssens a djective mood scale reduced from 25.8 before T1 to 13.3 after T3), perceptions of pain (mean sensory pain perception scores reduced from 18.8 at T1 to 15.2 at T3) and the ability to cope with pain (mean affective pain perception scores decreased from 29.8 at T1 to 21.3 at T3), from repeated applications of therapy $(P<0.001)$. Completed to build on existing qualitative findings, this study sought not only to identify effects but also assess the viability and potential of a larger scale, randomised control trial which the authors hoped may follow. The limitations of the design, identified by the authors as lack of representativeness (single centre and only 1 therapist) and no long-term follow up (only directly after the intervention) left many questions unanswered, which was apparently acceptable, however this meant that while it was clear benefits were derived, it is not clear how or why benefits were derived. In order to further explicate specific study effects, the authors explored previous trials that have included other components besides this particular approach, such as 'classic massage' (a different technique without solum oil embrocation), the pharmacological effects of lavender oil, and also placebo effects (which they posited may include expectation, self-selection and the setting of the trial).

There are a number of issues for me here. First, the working definitions of CAM that overarch the trial: the researchers here seem to define the therapy and responses by what it is not, rather than what it is. I find this confuses and devalues the whole of the therapy approach and prevents the reader from engaging deeper, and other therapists from transferring findings, into their own practice. Second, the design of the study and the detail of the reporting raises a number of unanswered questions: specifically minimal details of the actual treatment were included, beyond it being applied by a single "trained and experienced study nurse" who individually adapted intensity and duration within a framework of 30 minutes therapy followed by 20 minutes rest (Ostermann et al, 2008:238). As a practitioner, I know there is likely to be more to the interventions (and even the participants) that the authors describe, and find it odd that they would offer the potential variables and their influence at the end, to challenge the value of their findings, rather than include these within the method and seek to analyse their impact in the results. I see this as a further act of disconnection between the reader and the 
practice delivered, which here is accepted as an unavoidable limitation that only a larger, randomised clinical trial could potentially assess (or control for).

To combat this, the authors seem to spend a large amount of effort in justifying the responses through statistical tests or making loose assumptions about any potential confounders, nonspecific effects or placebo response. This suggests to me neither a holistic nor authentic approach to CAM research, which could have involved actually describing or engaging with the selected therapy from either the philosophy of anthroposophy (the system to which rhythmical massage belongs) or the actual 'lived' application/experience of this, thus serving to close the gap between research practice and therapy practice, such as I had witnessed it in Berlin. Perhaps, even more frustrating, was the clear message from the authors that their findings were unreliable unless/until they were supported by a quantitative study.

Continuing through my own data collection, a later prospective observational study of homoeopathy in cancer care by Rostock et al (2011), shows a continued effort to prioritise the 'scientific method' of research above the clinical or therapeutic methods involved. Their motivation for this was to 'do better' the scientific method than previous studies of homoeopathy in cancer care, which the Cochrane Collaboration of reviews have found littered with mixed results (Rostock et al, 2011:2). From the outset, the study authors separate the philosophy behind the mode of action from clinical effectiveness (seeming to assume there is no plausibility to this) which raises, for me at least, their rationale for selecting the method at all and trying to shoe-horn these into a design that could not substantively be achieved.

Their approach had been to observe existing practice in 4 clinics ( 2 offering homoeopathy and 2 conventional cancer care) and compare results between the sample in terms of quality of life, psychological wellbeing and fatigue outcomes, and thus excluded other potential effects. The treatment approaches were applied as they usually would be in the daily practice of these specific settings, while the methods of study were guided by a strict protocol, rather than philosophy, with the sample of participants and clinics included rigorously selected as "strong exemplars of the treatments in question" (Rostock et al, 2011:7). Again, there was clear benefit evidenced of homoeopathic care over conventional care, despite the fact that many of the homoeopathic patients arrived into the study later than conventional ones (having often been through the conventional route first), and so were often more ill than their counterparts. Similarly, the treatment regimes of the conventional patients carried more unpleasant sideeffects than the homoeopathic care, also skewing any results. 
While I am pleased to see the authors so ught to explore homoeopathic care as a whole, rather than isolated aspects within this (such as remedies or consultation styles), I am again troubled by the assumptions and culture of research here, which fails to engage with the underlying philosophy of classical homoeopathy (beyond briefly identifying aspects of this at the start) or at least consider the mechanism by which homoeopaths might explain the participants' experiences or outcomes. To me, this signifies the best and the worst of the scientific agenda and the rush towards EBM.

Given the issues raised by the above studies, it is unsurprising that conclusions of "higher order' research, such as systematic reviews or meta-analyses, deem findings inconclusive in terms of outcomes or advice. Despite best efforts, there is always something more that needs to be done first, which was also the conclusion made by Wilkinson et al (2008), in their systematic review of massage for 'symptom relief' in cancer patients. Initially locating 1325 papers, from these just 10 were identified as suitable for inclusion. The focus of the evidence was to "assess the evidence of massage (including aromatherapy massage) in improving physical and psychological well-being in patients with cancer" (Wilkinson et al, 2008:431).

Reporting of the actual interventions was extremely limited and suggested nothing that, as a massage practitioner, I could relate to. This made the review, however impressive it sounded, of very little use to my practice. Similarly, the review of the results was inconclusive. However, I would question the motivations for this review, prior to any other critique. Massage therapy, as it is commonly understood, has no philosophical basis in cancer treatment and seems to have been reviewed because it is a popular choice for cancer patients. First, I question the issue of choice - it may be that, due to cultural discourse, the most accessible CAM methods available to the general population is massage. Moreover, as a modality without a significantly different philosophy of health and illness to conventional medicine, this is more acceptable adjunct to conventional cancer care. Different populations may have different choices. Second, I question the outcome measures explored by the reviewers; all validated but not those that test the common intentions behind the selected therapies. Also, the authors considered the details given about the individual interventions to be "generally clear" (Wilkinson et al, 2008:437), but their judgement here is does not appear to be supported. I would also contest the assumption that any outcomes reported could only be attributed to either the identified modalities (rather than their style(s) of application) or the properties of individual oils. 
Due to the range of variability in massage techniques, therapists and patients and cancer sites, the authors concluded that a meta-analysis was not possible. Moreover, many studies were excluded from the review because of their methodological issues: no control group, no baseline assessment, no patient generated data from validated outcome measures, not randomised, and therapy not delivered by 'qualified' staff (which means those included did meet these criteria). This gives a very clear impression that data recovered from studies is only of value if it meets strict criteria, which may have little to do with either the actual therapy or its underpinning philoso phy.

The authors conclude that more studies of higher methodological quality are needed before any evidence-based decisions can be made. I see this 'stock response' as quite a narrow 'get out' for the researchers involved, and find it is also the theoretical quality of systematic reviews that needs attention, so that valid questions can be asked in both empirical research and reviews at the outset. I see here a very clear, and startling, issue of priorities and the valuing of one set over another, which says more about power and discourse than it does harmony and a uthenticity.

Even more recently, McCall et al (2015) have implemented a small, qualitative study exploring the patient experience of Yoga in improving cancer symptoms. Conducted with just 10 patients, data consisted of in-depth interviews which were analysed for themes and patterns. The primary findings were patient perceived benefits in psychological and emotional wellbeing, physical wellbeing and body awareness, social cohesion and group support from peers (McCall et al, 2015). In addition, Yoga users reported a greater sense of personal responsibility for their health and outcomes and a sense of connection and calmness in their mind and body. For many, it was neither possible nor desirable to attribute these responses to a single factor, rather the combination of all factors which reflects the holistic ethos of the practice.

Conducted after my own, but not too late to be included here, I found this study more meaningful to engage with, both because there is a philosophical basis for yoga in cancer care as the discipline forms part of the larger parent system of Ayurvedic medicine, and because it sought to uncover whatever patterns and outcomes were naturally present rather than seek evidence for preconceived ideas. Thus the data was able to yield more information and more quality than an alternate narrow checklist could account for. These included the value of expert in-person instruction; breathing techniques and meditation specific to a cancer group; the ambience created by instructors; and the importance of the yoga space as a factor in their 
enjoyment and adherence to the programme. Indeed, the authors suggest this last finding (the ideal yoga space or ambience) to be a valuable area for further study, positing that the size of yoga effects in clinical trials may be underestimated if yoga has been delivered in less than favourable conditions. Participants here clearly noted having a non-medical environment as critical to their healing process. Some differentials between types of yoga practice were also indicated and discussed, which I found provided potential resonance for the reader (if they have also experienced or have awareness of Yoga).

This approach to research appears contrary to the 'accepted wisdom' of conventional discourse that argues for larger (not smaller) samples and more (not less) statistical significant outcomes, yet seems to me to offer increased validity in terms of the model applied and relationship to underpinning philosophy. Indeed, it is notable that these authors did not conclude by suggesting such studies follow next, but rather advocated more qualitative work to compare, support or widen their findings. I found this style of approach, unlike those demonstrated within more quantitative designs to create the potential for also narrowing the commonly observed theory-practice gap in CAM research. However, one thing still bothers me. The study authors and participants never seemed to discuss the diagnosis of cancer from an alternative or holistic perspective and were reported as receiving both conventional and complementary care, for their cancer. This may suggest an underlying concordance with the dominant perspectives of cancer as something to be treated, although yoga would perceive the person as needing treatment. The influence of this potential bias is not explored in the study.

In summary, through this brief review I found my primary concern to be with the focus of EBM research (always on what can be measured rather than perhaps what should be measured). As long as this continues, this means there will inevitably be gaps within the evidence produced, which then adversely affects evaluations. Even more than this, I have become troubled by the ways many researchers seem to be locked within a discourse (or lock themselves within this) that, while they admit is not working, do not seem to want to challenge.

This was almost an echo of what I had noticed from my engagement and discussions in Berlin (and also later events I attended in Bristol, Manchester and Vancouver): that the majority of active CAM researchers appeared 'persuaded' that becoming more 'mainstream' in their practice was the way to progress CAMs. Among this 'research community' I was fast feeling and looking like a 'lone voice'. This caused me some concern and made me question the ability of any 'counter-argument' to be heard. 
The overwhelming impression from these studies is also that the underlying intention is to discover the effect of treatments on health conditions which is often not the original intention of therapies. It is neither my education nor experience that CAM practitioners typically apply their arts to treat health conditions although, influenced by the dominant discourse, it is likely that a number of practitioners may be drawn to applying this non-traditional approach in practice, to avoid being marginalised and increase their accessibility to patients (similarly influenced by dominant discourse and only amenable to therapies that match). So too, researchers may become misled into trying to evidence this new model and intention.

This suggests to me that there is so mething wrong within current research approaches. Why is no-one studying (or publishing studies) CAMs that aim to restore balance, given that within vitalistic medicine (which many CAMs fit) a fundamental principle is that symptoms are simply a manifestation of imbalance and a correction of balance will result in recovery of the person? Why do the therapists who participate in studies modify their natural approaches to treat conditions rather than people, which is where the strengths of many CAMs lie? Why are researchers in CAM not doing more to challenge the dominant discourse in this?

All questions that are not easy to answer, I felt that neither would the course of my research be - not if I wanted to draw real meaning and bridge the research-practice gap I identify (similar to the theory-practice gap noted by Mulhall (2001)). What I did find clear was that as long as other researchers continue to pursue the 'objective' agenda, subjectivity both of those delivering and receiving CAM therapy would continue to be overlooked in evaluations of therapies and their outcomes. I maintain that this is a vital ingredient in understanding how any non-pharmacological intervention may be of benefit, and that consideration of this may significantly improve the validity of studies and interpretation of such findings. It may also encourage/enable therapists to think more deeply (or holistically) about the potential limitations of the care they provide or the potential freedoms they create.

\subsubsection{The placebo effect}

I cannot move forwards from trials without also discussing the concept of placebo. I find that within the literature, much of the knowledge surrounding this is not knowledge at all but opinion and conjecture, which I want to explore further given the importance placed on this within trials and the inherently non-pharmacologic nature of many CAM therapies.

Both within the designs used, and to explain 'positive' outcomes in the absence of local theory, placebo has become a firm fixture within the CAM literature. A contentious concept, this was 
originally developed to assess the effectiveness of pharmaceutical agents, and has become either a necessary arm of any clinical trial; a confounding variable in need of control or elimination; or even a means of dismissing CAM therapy effects. Outside pharmacological trials, many people use the term placebo to mean a wide variety of healing effects, as distinct from drug-like effects, including factors such as expectation, interaction with the therapist and even the thickness of the carpets (White, 2000). Discussions about these context effects often begin and end with the consensus that any outcomes are the result of 'placebo' and thus ignore other potential explanations about the natures of interactions.

Examining the term 'placebo' establishes some important context. Beecher (1955) in "The Powerful Placebo" was the first to report the observation that $35 \%$ of patients will achieve positive outcomes from 'placebo treatment'. Originating from the Latin meaning to please, this was used by Beecher and his counterparts to describe any intervention that was nonpharmacological in nature yet provided positive or pleasing effects and outcomes. In its essence, this could be used to describe many CAMs today, however, perhaps due to some of the phrasing choices within this original paper, modern use has since modified use to become synonymous with anything that is dummy or fake (in terms of either interventions or effects). Beecher, it seems, found it too complex to keep referring to dummies and placebos as separate arms, and so decided it would be simpler "to use the one term" regardless whether the interpretation "falls a bit short of precision" (Beecher, 1955:1602).

I am unsure whether what I find most shocking is the fact that succeeding researchers and practitioners seem to have unquestioningly accepted the merging of the terms 'placebos' and 'dummies' as the same phenomenon without ever thinking about it, or that many of those who seek to unpick and understand the placebo effect, for better application and use, have often never read this original paper so don't connect their modern acceptance with the origin of the word (personal communication with various current CAM research leaders and academics). Either way, the impact of this requires noting.

Moerman (2002) suggests this may point towards a cultural dependency of meanings and values ascribed by patients, whichever discipline (orthodox or alternative) they originate from - simply that it may be disorientating for the newer meaning to be challenged or refuted. I would suggest this goes beyond the patients, with many practitioners and researchers similarly infected! Indeed Helman (2001:6), in his discussion of placebo, specifically draws attention to the physical setting where healing rituals take place, where he argues strongly for the multiple roles of physical, cultural, social and economic contexts as making clear but subtle 
contributions "to the totality of the healing process - and thus to the placebo effect as well". I certainly take the point that physical setting and healing rituals can contribute to the healing process but cannot so easily equate the healing to the placebo effect without first considering the theories of subtle energies and etheric or astral forces as mediators of these. Aside from the fact that Helman (2001) doesn't differentiate between CAM modalities, this perspective effectively denies any therapeutic effect from the intervention, without any data to judge this opinion.

This begs the question that for any therapy to outperform placebo, must it also outperform cultural opinion? I see little potential of this anytime soon, as aside from not appearing keen to question the appropriateness or validity of the concept within CAM practice or research, many authors fail to note that placebo is a theoretical construct originating from within biomedical and pharmaceutical discourse, with its use in relation to context or meaning effects a theoretical construct also. This suggests something about their originating paradigms and influence of discourse, as well as the ability or willingness of researchers to challenge popular beliefs where they do not fit with CAM theories and philosophies. Coming back to my premise of 'space', it may also say something about the remits and/or limits imposed on researchers if they do not have the space or support to explore this fully.

Yet placebo research is fast becoming a distinct discipline within healthcare research, with some authors even trying to harness its positive healing effects. Ernst (2001) for example, remains unequivocal that placebo effects do exist, also writing about the difficulty in defining placebos, explaining their modes of action and/or maximising their potential. In a theoretical rather than pragmatic mood, he further equates these to the various non-specific meaning or context effects around CAM treatment (including physician attention, the healing setting, and positive expectation) and even declassifies much of CAM practice as inert or placebo. I suppose, harking back to Beecher and the original pre-definition, this is true but the term may be too simplistic to be considered precise.

Others also, seeking to explain the general acceptance of healing effects achieved by the various complex components of holistic CAM interactions, as synonymous with the term 'placebo', include Kienle and Kiene (2001:47) who attempted to validate the 'placebo effect', basing their view that placebos may have active components not in consideration of actively observed 'context effects' but on a general lack of evidence to their inertness and suggest that dismissing any aspect of therapy simply as placebo represents failure on the part of research, to explore "effects that we do not understand". Previously, Joyce (1994) has questioned the 
practical utility of trying to separate the specific from non-specific effects of CAM therapies for the sake of evaluation, instead arguing for a more systematic and less chaotic approach to the whole issue, while De Craen et al (2001) and Campbell (2000) continue to argue for more information about the characteristics of non-specific effects, suggesting these define the effects achieved from CAM interactions.

Stressing the need for more studies, particularly given the growing public interest in CAM, Vallance (2006) puts forward a number of mechanisms that may influence the production of placebo responses, cautious to note how research has yet to adequately trace "the complex web of interacting psychological and physiological processes" that underlie this effect (Vallance, 2006:293). Adding to this, Kaptchuk et al (2009:386), has suggested that "activation" of a patient-healer encounter include the mechanisms of cognitive factors; emotional factors; practitioner behavioural factors; patient behavioural factors; sensory factors; aesthetic factors; and symbolic processes to generate effect. Unfortunately, there is no theoretical frame as 'how', beyond Clow (2001) who firmly lays placebo effects at the door of 'belief'.

Aside from these arguments, the reality is that efficacy within healthcare trials (whatever the modality tested) has become a matter of judging 'active interventions' against placebo ones and exemplifying the dominance of RCT discourse across all healthcare disciplines. Testing CAM interventions against placebo in these situations could, according to some of these opinions, be a matter of judging one placebo against another with no guide to ensure either fairness or cla rity in delivery or interpretation.

Wherever the potential catalysts for positive healing responses in therapy originate - the intervention, the practitioner-patient relationship or the healthcare setting - nowhere within the placebo literature is it considered that the synonymous linking of terms placebo and CAM may be placing constraints on potential inquiry into the 'dynamic mechanisms' of healing effects. This is despite the historical introduction of 'placebo controls' as a political concept, to constrain unconventional healing practises in $18^{\text {th }}$ century Paris, the reby controlling scientific power and access in society (Myers, 2010). Then, as now, reported outcomes were of lesser concern than the claims made by which they were achieved, with modalities deemed invalid if no causal link between intervention and results could be evidenced (Myers, 2010:1301). 


\subsubsection{CAM surveys and social research}

A more populated and popular avenue of CAM research, prevalence and attitudinal surveys sit alongside ethnographic and other qualitative methods in making up a large proportion of current CAM research. Acted to evidence the need for trials (based on number accessing therapies) or to illuminate trial findings (exploring aspects noted but not evaluated), these studies tend to focus on aspects of the therapy experience beyond intervention: so people, place, econo mics (and other contextual variables).

Assessed in the light of issues raised through the trial data discussed, I want to further explore my two main areas of interest and review the relevant literature to gain a general feeling or appraisal of the mood in relation to this: the active role of ambience or space and the therapeutic relationships that people form, within these.

\subsubsection{Healthcare environments}

Providing the fundamental backdrop for CAM performances and healthcare delivery, the physical and dynamic qualities of 'space and place' are both part and product of the underlying context, yet very little is known (or reported) about either the composition or impact of these in therapeutic care. Traditionally the domain of geographers, architects and anthropologists rather than healthcare researchers, appraisals of CAM therapeutic environments often focus on the impact on patients and outcomes, with few considering the impact on these on the quality of care practitioners deliver (as a mediating factor?).

Almost exemplifying (again) the discourse around EBM, much of the data currently on the healthcare environment in CAM has come as a by-product of other clinical, qualitative studies. Examples of this include Di Blasi's and Kleijnen's (2000) study of clinical consultations at the Glasgow Homoeopathic Hospital, where the focus of the study was actually the consultations, but patients reported the 'atmosphere' of the hospital setting as also being active within their 'healing encounter' anyway. This finding was neither identified nor explored further, presumably because it was beyond the brief of this work, although did feature again in a follow-up study at the same hospital by Mercer and Reilly (2004). Here, the researchers found the physical aspects of the setting, in particular the feeling of space and light, resulted in a high incidence of reported positive effects on patients and staff but again fail to explore (or even describe) anything beyond this, directing those interested instead to photographs of the hospital on the website (www.ghh.info). 
Also revealing unexpected value to the treatment environment, Long (2007) had only sought to explore the effects and experience of shiatsu treatment, so failed to include any detail, description or imagery. Rating this as being overwhelmingly positive, presumably the author had not considered the environment to be a part of the treatment prior to entering the field. Smith et al (2009:184) also reported 'therapist attention to the massage environment' as positively enhancing therapy and noted a "relaxing atmosphere and warm towels" but again did not think to record or share any further details.

Boon and Kachan (2008) in their comparative ethnography of integrative medicine clinics at two Canadian hospitals had perhaps greater opportunity than most to explore the material (and dynamic) presentation and influence of context and setting in therapy. Their primary remit was to highlight key issues in the development of two integrative medical clinics and these included, but were not limited to, the physical setting. In a practice that turned out to be a play of power and dominance, whereby CAM therapies needed an inside 'champion' to ensure their survival (and also send out a message of CAM as 'credible' - which actually is also a survival strategy), they noted specifically that the physical space allocated to the therapists for therapy delivery, and the limitations placed here on their normal scope of practice and service, were found to impede the therapists' ability to do their work but stopped short, again, of any descriptions which could have further informed on the nature and nuances of this experience, for practitioners and patients.

Illustrating that evaluation studies consistently find the setting to be an important factor in the experience of delivering and receiving care but that, for whatever reasons, the researchers never seem to develop their observations into an evaluation or analysis that may actually enhance engagement with the data present, is an important observation. This concerns me, as I do not currently see matters moving forwards unless researchers begin to actively look in the right places and start to see or value what may be there.

Freshwater (2005) has suggested this could be an important area of activity as place and space can be invested with all sorts of meaning, and we display our intent in the ways we compose and fill our spaces. Mull \& Mull (1983) in one of the few in-depth, descriptive pieces about therapeutic environments available, illustrate this most clearly. All data result from a single interview/visit to the home and practice of a Mexican American folk healer (Curandero). The authors depict in great detail the design, décor and artefacts present in the treatment room, alongside the physical layout (local area, external environment and internal office space) and the healers 'attire' which, presented in a narrative style, paints a 'picture' of the encounter so 
vivid that this whole 'scene' is re-created in the minds-eye of the reader. From this, it is easy to engage with the authors and see where qualitative inferences between philosophy, practice and outcomes are then made. Clear also is the impact of the setting and the practices therein on the researcher's perceptions of the practitioner and his work. These, like any treatment, create a holistic performance of the personal world views and application of the practitioner which can then be evaluated as a whole. This seems to me to be a good way to go about this.

A similar approach is taken by Kleinman (1980) in his study of Traditional Chinese Medicine healers in Taiwan and Boston. Here, detailed descriptions of both the setting and the practices are supported by a catalogue of photographic images that make it possible to both imagine yourself in this performance of therapy and also to connect with the role and value of the setting for it. This comprehensive text goes much further into the topic of place and performance of traditional medicines than any other attempts I have found, and offers both explanatory models and a historical background to the study of cultural healthcare. Kleinman (1980) even draws the parallel between the decline of holistic conceptions of such relationships (place and therapy) with an observed decline in the holistic conception and practise of medicine.

It is notable that both this text, and Mull and Mull's (1983) report, are studies from over 30 years ago while the others cited so far hail from within the last 15 years. This places the whole of my inquiry and the general current approaches to CAM research into a fresh context. This is not to say that there have not been studies seeking specific evidence on the influence of setting in CAM 'private practice'. Turner et al (2007) set out to reveal patients perceived importance of environmental factors using a questionnaire method (thus without either direct or intimate knowledge of the individual setting to which respondent reports relate). The authors revealed that 'presentation' of these healthcare settings is, like those discussed by Mull and Mull (1983) and Kleinman (1980), embedded with messages about unique and local attitudes to both the patient and the philosophy of healing, with patients expressing an increased confidence in encounters as a result.

Those who come closest to examining the nature of interaction between place and healing are not (perhaps unexpectedly) from within CAM, but from mainstream healthcare, health geography and environmental psychology. These overlapping schools seem to easily identify and interpret the synergy between natural, built, symbolic and social environments in promoting healing: each from their own philosophical perspective. They acknowledge the unique elements of place, history, culture symbolism and memory in the event with results to 
date influencing some modern healthcare provision and design, which suggests a far more progressive approach to understanding outcomes than many alternative or holistic efforts.

Exploring this issue with great awareness, Shur Bilchik (2002) builds on Ulrich's (1984) publication about the role of a hospital window view on the post-surgical outcomes of patients (where those recovering in a hospital room with a natural window view versus a brick wall had shorter hospital stays and fewer analgesics), to cite many different examples of the ways that hospital (spatial) design can impact the lives of those who inhabit them. Arguing most convincingly that health 'care' cannot be separated from the environment where it is delivered, Shur Bilchik (2002) reveals interest in the fabric and design of mainstream clinical environments to be a serious issue, particularly in the USA, where hospital lea ders a re realising that reduced costs and improved care can result from a pleasant, efficient environment

However, she also notes that qualities of the setting that underpin therapeutic interactions are rarely attributed in encounters that are perceived as biased on the characteristics of the patients, the practitioners or the interventions themselves. From a holistic perspective, viewing therapy in isolation from the context or setting that 'holds' it may provide a false distinction and actively distort findings by denying role and value to these production parts. Indeed, growth in intervention studies may be a factor in perceptions of CAM as independent of the context in which they are delivered.

Sloan Devlin and Arneill (2003), in their paper on healthcare environments, illustrate the various ways that individual design factors such as architecture, colour, light, sound, view, air quality and control can all be manipulated to directly impact health outcomes. For example, they suggest lighting and colour can enhance mood and reduce patient disorientation, while proximity to nature elements, or images of nature, can reduce blood pressure and anxiety, increase muscle relaxation and facilitate reactions to stress. Not appraised synergistically, they still conclude that research into 'Evidence-Based Design' has the potential to improve patient well-being. This assertion is similarly supported by Leather et al (2003) who, in a comparative study of hospital waiting areas (traditional and nouveau) that were different in both functional and aesthetic attributes, found the nouvea u environment rated as significantly more colourful, positive, stimulating, attractive, relaxed, comfortable, cheerful, motivating, pleasant and open than the traditional resulting in improved mood, altered psychological state and greater satisfaction. Coming from mainstream discourse, the authors suggest that value from an attractive environment within hospital design may lie principally within its ability to distract attention and moderate mood and behaviour. Ridenour (1998), reporting on personal 
accounts from patients, visitors and staff who have experienced the benefits of art enriched environments, similarly relates the value and therapeutic potential of creativity and arts within healthcare settings to the ability to 'distract' attention from the experience of illness while suggesting that intent and intertwining of spiritual and community beliefs may also have a role. While the degree of true impact is currently unknown due to the difficulty of science's traditional measures in quantifying this I believe a CAM perspective, based on theory and philosophy, could likely add several more layers.

Presenting one of the few studies to focus on providers rather than recipients of care, Armstrong et al (2004) showed positive hospital design to play a role in the performance of NHS nurses in England. This large scale mixed methods study found that interior design matters, particularly to nurses, impacting their whole workplace performance. Thoughtful use of lighting and colour were again highlighted as of particular importance and nurses reported feeling positive, when they had influenced design choices, and dissatisfied when their ideas were not taken on board. Making for a happier ge neral environment these results were felt to impact both nurses and patients in their 'shared experience' of the environmental setting, while the design and layout of space suggests both facilitating and constraining roles, in regard of management and teamwork. This echoes the earlier work of Biley and Freshwater (1998:99), who have argued similarly that hospital and other health care environments could and should be "nicer places to go", suggesting the difference that gardening and arts programmes can make in humanising the space for all.

Taking this argument also 'outside', Bell et al (1996) offer a whole programme of studies by Ulrich $(1979 ; 1984 ; 1986)$ and Ulrich et al $(1991)$ to evidence the fact that restoration most naturally occurs in natural environments, and that urban settings generate no comparable positive response (Bell et al, 1996). Whether the sense of 'restoration' in certain environments is a psychological response (Bell et al, 1996:40) or some other mechanism is unclear as the text originated from the interpretations [and bias] of the text's authors.

Saunders (2003) very clearly relates the impact to subtle energies, in particular electromagnetic radiation, for the relationship between humans and environment whether natural or urban. Identifying patterns of 'interference' or 'resonance' in the intersection between "terrestrial energy fields and cosmic radiation originating from the Sun, Moon, Planets and Milky Way", he explains that modern building construction (in particular modern hospitals) can be responsible for creating negative health impacts. Also described as geopathic stress, this is related to the earths' natural magnetic field, suggesting that forces of 
electromagnetic radiation have the capacity for great impact on humans and their health, often unseen and without boundaries. Freshwater (1997) having also written on this matter, claims these forces create impacts that are both negative (headaches, depression, allergies, cancers) and positive (vitamin D from sunlight) for our health, and posits that constructed setting or design of buildings does not act alone, but as a conductor of electromagnetic energies. This may explain part of the phenomena within 'sick building syndrome', whereby the building suffers, along with the people within it (Freshwater, 1997:161). I would add to this that they, like the content of this review, are also a product of their agents.

Following this theme further, Gesler (2003) suggests it may not always be apparent why places heal but this does not mean that a place cannot have healing capabilities. Having determined, through his own humanistic exploration, that 'healing places' originate from four distinct, yet interconnecting 'environments' (relating to the natural, built, symbolic and social dimensions of place (Gesler, 2003:2)) he goes on to illustrate that introducing elements from the various 'environments' may enhance the potential for healing to occur within modern day spaces (Gesler, 2003:92), using examples of places that each enjoy reputations for 'healing'. As Biley and Freshwater (1998:98) observe, early hospitals were often located in "special places known for their healing qualities", while moves now towards individualised patient-centred care has brought a disconnection from the aesthetic qualities of enviro nments, with economic concerns given greater value and "high tech, stainless steel sterility" the new ideal (Biley and Freshwater, 1998:99).

Taking this into CAM encounters brings an additional dimension to the analysis of context, in the experience of health and healing. Moving beyond both the descriptive and experimental approaches of how the design and components of the treatment setting or relationships therein enhance healing, belief and 'placebo' effects, health geography is an additional discipline that advocates focusing on the broader geographical perspectives of health inequalities, the built environment, and psychological attachments of people to space and place. Indeed, the construction of these different environments may impact outcomes by telling people how to behave and experience the events therein, a perspective similarly established within performance tourism (Edensor, 2001).

Adding an additional 'other voice' to the body of evidence, and thus 'framing' experiences of healthcare (Andrews \& Moon, 2005a) and healing (Gesler, 2003), offers the potential for a richer 'contextual perspective' for other more clinical, practice based data to locate in. However, there remain many different geographical strands that CAM research has so far 
failed to engage with, despite their implicit role within the design and delivery of CAM therapies. These significant gaps in the holistic understanding of CAM practice as a social and cultural phenomenon offer new avenues for both the design and interpretation of research in this field.

Perhaps due to greater freedoms afforded by not being the 'challengers', research within mainstream healthcare seems here to be more holistic than the CAM alternatives, who remain stuck on the treadmill of 'evidencing efficacy beyond placebo' instead. I find it curious that, given many CAM interventions will either claim or aim to impact health at the subtle energy level, CAM studies that have actively considered the issue of 'subtle space' or 'human place' are notably absent from the literature. Maybe, in order to truly engage with the discipline, CAM researchers should learn from the mainstream rather than push against it, taking both the right and responsibility to put the interests of our own patients and practitioners before those of policy makers and 'the establishment' to define for ourselves the evidence we seek for our practice.

This is a point raised by Gallagher (1993) that is as relevant now as when she made it. Summarising the (then) current knowledge on the various impacts of subtle geophysical energies on human health, she noted how the absence of evidence does not equate to evidence of absence, and cautions the role of the research model used as a dependent factor. This may be something to try to hold onto, a bit more.

\subsubsection{Relationships and Rapport}

Perhaps the other most commonly explored aspect of CAM practice is the therapeutic relationship between practitioner and patient. A most critical component within my own practice, where interventions are 'non-pharmacological' in nature, I often actually broaden this to the relationships between practitioner and therapy, and also between patient and therapy, as I feel these to be interlinked.

Offering what may be considered a core text on the matter, Mitchell and Cormack's (1998) review the current theories and evidence in CAM conclude that a good relationship is central to healing, with the partiality of the practitioner and their ability to both understand the needs of the patients and meet those needs often making the biggest difference (Mitchell and Cormack, 1998:156-7). This is echoed by Long and Mackay (2003), who point to the value of a good resonance between characters as a factor in effective relationships within their exploratory study of shiatsu in the UK and Germany, where clients felt having confidence in 
the practitioner was important to get good effects or positively enhance 'therapy outcomes'. A follow- up study, conducted with participants in Austria, Spain and the UK (Long, 2007), similarly found the client-practitioner relationship to hold therapeutic significance, revealing that clients were overwhelmingly positive about their relationships with their practitioners, feeling both 'listened to', and 'accepted' by these 'trustworthy', 'skilful' and 'warm' individuals (Long, 2007).

Positive expectations regarding therapy were also a factor in enhancing patient outcomes in a study of Natural Therapists (Dellmann and Lushington, 2012). Focussing on primarily interpersonal skills, the authors discovered that therapists can generate such expectation by "putting a positive spin" on the patient's own potential, the potential of the modality used (although without making false claims) and emphasising improvements achieved at follow-ups. They also hypothesised that the private nature of fee arrangements could play a role in the deliberate use of expectancy. Similarly Leach (2005) found the ability of these characters and their successful employment of skills and strategies a cornerstone to building good rapport, which in turn generates good therapeutic alliances and strong therapeutic relationships.

Ackerman and Hilsenroth (2003) saw, in review of therapist characteristics, that specific interpersonal qualities (trustworthiness, experience, confidence, lucid communication and accurate interpretation) could positively enhance therapeutic alliances manifest here through practitioner investment with the client (enthusiasm, interest, exploration, involvement and activity). Descriptions of therapists as subjective, engaged, helping and holistic in their approach to healing work has also been noted by Carter (2003), while good rapport was near the top of the list for many patients when Smith et al (2009) asked about factors they value within massage therapy encounters. Participants were clear to note that alongside factors including trust; therapist enthusiasm; knowledge and expertise/skills; professionalism; and warm towels that any importance (or crucially benefit) from practical aspects of the intervention would be lost if they didn't also feel comfortable with the therapist. This appears to support the view of Paterson and Dieppe (2005), who consider that elements such as patient expectation, manner and consultation style of the practitioner and the therapeutic setting, often categorised as incidental, may actually be integral to therapy practice and outcomes.

Attempting to quantify this, Harris et al (2010) sought data from a single complementary therapies clinic in South Wales using two separate questionnaires, and assessed the quality of the relationship between client and therapist in relation to clinical outcomes. Results showed 
a strong working alliance but correlation of scores, between clients and therapists, was of low significance due to many clients not seeing the same practitioner at each visit (this variable was not 'designed for'). Sharing and partnership building within the delivery encounter (cognitive-emotional care) have, however, been shown elsewhere (Di Blasi and Kleijnen, 2000).

While this makes a pleasing read to any therapist who feels these attributes describe their practice, my main critique is the lack of any coherent philosophy linking these ideals to actual therapy practice. Displaying different levels of interconnectedness, the relationship between characters within a shared plot may relate not only to therapy outcomes but also to the underpinning contextual synergy that creates the nature and experience of these performances. Where, for example, is the theory that supports the practitioner's chosen consultation style? What outcome do they intend from warm towels and do they get it? Is there a shared 'meaning-making' that supports a strong working alliance? How do therapists generate trust and how do they keep it? What is skilful? Without deeper consideration and analysis, these results (like those within the earlier described trials) can fail to communicate with practitioners or researchers in any meaningful way, removing the opportunity for development.

A rare reflexive piece shows Lees (1997) seeking to determine those external factors within the wider context of practice that can either positively or negatively enhance therapeutic relationships. Focussing on primarily social and interpersonal aspects, he assumes responsibility for his own individuality, concluding the central factor to be his own ability to enact "the principles of adaptation, individuation and homeostasis" (Lees, 1997:47). However, even he stops short of locating his 'performance' of these principles within the physical and dynamic qualities of the 'space' where he finds himself. Disconnecting one from the other, even though they were likely 'dramatically' if not dynamically interlinked, he limits his full potential to engage and thus communicate as multi-dimensionally as may otherwise have been possible.

Just considering that aspects of this performance (therapy or research) may have some dependence upon the context of the production, demands a closer relationship between theory and practice, for therapy and research. 


\subsubsection{CAM issues in research and methodological critiques}

Highlighted by many, but addressed by few, the problems in CAM research are often debated but never seem to be resolved. My reading of this comes down to the context of research and researchers, and the challenge of trying to achieve successful outcomes within this.

\subsubsection{The challenge of complex interventions}

CAM itself is characterised by an innate variability in practises and an emphasis on holistic appraisal. This complexity raises significant challenges, which are also becoming a shared phenomenon across healthcare disciplines, so it is with some surprise that CAM research does not currently reflect these qualities. In a clear lack of congruence, many individual studies do not either value or publish their contextual data, demonstrating an inability to engage holistically with phenomena and limiting the value of such work. So me variables within CAM practice have been explored, however a dearth of studies remains that explore the whole of practice, thereby limiting understanding of variability on the experience of therapy and patient outcomes. This absence suggests a research-practise gap previously unexplored anywhere within the literature, and provides a starting point to consider the adequacy of existing approaches to researching CAM and the knowledge this produces.

Similar to the 'theory-practice gap' identified by Mulhall (2001), the gap between research evidence and the actions of individuals in clinical practice may be a key factor in the current failure of quality research in CAM being realised. This is not to deny the value that can be found in study of the parts, but that there is potential damage in failing to see how the parts contribute to 'the whole'. Articulating a more holistic perception of both the influence and impact of paradigm in a research context, and how these distinctly relate to modes of therapy within CAM, Cassidy (1994) offers something of a seminal text in this debate.

She explains how enacting a holistic paradigm benefits research within CAM and argues that 'paradigm fit' brings both the aims of the therapy and the research into alignment with one another and "able to see options that may be unapparent to reductionist thinkers" (Cassidy, 1994:11). This seems obvious, and yet so often research appears to be conducted without paradigm fit. Asking inappropriate questions and yielding answers poorly equipped to either resonate with, or influence, policy and practice means that implications for evidence are almost inevitably negative (Golden, 2012). This may be more of a symptom than cause with the underlying mismatch between the paradigms of practice and the paradigms of research limiting how studies engage with the heterogeneity of practices, the heterogeneity of 
practitioners, different explanatory models, variable training standards and variable regulations, as illustrated by Carter (2003); Zollman \& MacPherson (2009); and Ritenbaugh et al (2010).

Performing studies without proper and due attention to paradigm fit makes little sense as this can serve to divert attention away from the really fundamental questions, which are the only way to open honest dialogues, so that critiques can focus on the quality of these unique modalities rather than the quality (or acceptability) of the evidence that is produced. Whatever approach is taken, and whether this is acknowledged or not, paradigm provides the starting point for all research performances and underpins the researcher in their practice perhaps even more critically than it does the topic of investigation or the philosophical positioning of the topic. This is the site where both conception and actions originate and, more than any other, provides critical influence over the epistemology and ontology enacted by those with roles within such cultural practice; guiding the selection of any subsequent research questions, selecting designs and ultimately potential outcomes.

Given this, one has to question the motives of those who seek to devalue the notion of paradigm or that paradigms have meaningful purpose when it comes to selecting research questions and designing appropriate methods within CAM, like Vickers (1996). Consistently confusing and interchanging the terms paradigm and explanatory model ( $\mathrm{I}$ have always considered the latter to sit within the former) he attempts to make the former redundant, leaving only 'the science' to make sense. Evidencing the very real influence of paradigm in his own performance of this argument, he further launches a scathing attack on those who would offer a more balanced discussion (Launs $\varnothing, 1994)$.

According to Kelner and Wellman (2003:10), a "holistic research strategy requires a broad view that can encompass elements of the healing process such as the role of patients, the impact of the therapeutic relationship and the non-technical aspects of treatment"; if these can be seen and engaged as parts with the whole rather than parts of the whole, then I agree.

MacPherson et al (2009) have previously argued that designs studying isolated components are inappropriate for potentially synergistic or multimodal interventions, like many of those within complementary and integrative healthcare, as this can distort these investigations, suggesting pragmatic or preference trial designs to better reflect the complex causality of therapies in the real-world, also combining this with evidence from different sources such as 
observational studies and basic science. However, there appears a real dearth of literature on how to do this better.

Guidelines from the Medical Research Council (MRC, 2008) have done little to improve the situation, with critiques instead dominating, full of questions but few answers (Hawe et al, 2004; Boon et al, 2007; Fønnebø et al, 2007). Indeed, this may help to explain why efforts to understand the values of contextual elements within therapeutic encounters have thus far been studied as isolated aspects, and not whole.

\subsubsection{Whole systems research}

An emerging movement called Whole Systems Research (Ritenbaugh et al, 2003) presents possibly the most advanced position on this. As with the case for paradigm fit, the authors here argue for a broader CAM research model to include relevant theory and diagnostics alongside technique, in a full real-world context and claim that "complex systems/network theories offer a more relevant epistemological framework for scientifically based research on whole systems of CAM than do mainstream reductionist medical models" (Bell \& Koithan, 2006:304) and urge researchers to find a way to enact this.

Embodying such holism, within research endeavours, may require more freedom and confidence than is perceived to be available to many. Even the latest recommendations coming from the CAMbrella project (Fischer et al, 2012), after comprehensive review of the many, complex issues and answers in developing clinical and epidemiological research in CAM, omit discussion of holism in the design of clinical research in traditional or complementary medicine, except as a challenge.

This challenge is one that the Whole Systems Research group (WSR-CAM) have spent a number of years trying to address (Ritenbaugh et al, 2003; Verhoef et al, 2004; Verhoef, et al, 2005; Aikin et al, 2006; Bell \& Koithan, 2006; Jonas et al, 2006; Verhoef et al, 2006; Ritenbaugh et al, 2010; Koithan et al, 2012; Verhoef et al, 2012; Walach \& Pincus, 2012), just as Gestalt (Humphrey, 1924) and General System theorists (Pedersen \& Shears, 1973) have before them in the field of psychological research. However, success here has been slow and beset by numerous additional issues such as: developing multiple levels of measurement and handling the vast amounts of data produced; the unforeseen need for more qualitative patient data and for provider data; incorporating diversity; researcher difficulty in moving beyond the biomedical model of research; realising the heterogeneity of systems, variables, conditions and 
patients; the outcome of expectation; great variability in practitioners; publishing constraints; and the role of principal investigators in determining research results (Ritenbaugh et al, 2010).

Perhaps a key to understanding these selected approaches is the 'likely audience' each is trying to please, whether this is patients, funders, employers or critics. It could be naivety on my part, but I cannot help feeling that it should not have to be this way! If the 'practice of CAM' is getting lost within the 'practice of research', this is of limited help to anyone, which risks the same outcomes here as nursing has previously found (Mulhall, 2001).

Jonas et al (2002) suggest that a balanced solution to the research-practice gap within CAM involves CAM research 'crossing' various 'bridges' to gain clinical acceptability and use within 'medical practice'. This again illustrates how the needs of stakeholders are currently not met, and suggests a number of areas for improvement, not least understanding the complex ways specific and context factors interact within practice. However, the principal recommendations are fragmented ones, with each providing a different 'piece of the puzzle' in the hope that, one day, a whole picture will emerge.

This is a frustrating situation as holism can be a tangible concept if researchers are sensitive to it and brave enough to pursue it. I am sure that many of those who conform to dominant scientific or reductionist discourse would prefer to describe themselves as holistic therapists or thinkers but the pressure of explaining or evidencing CAM therapeutic effects in a form deemed 'acceptable' by those responsible for publishing such studies, can serve to mediate or block such acts, presenting a real barrier to improving policy and provision in this field. This may be a matter most urgently in need of review if either/both the quality and validity of future studies in this field is to be raised.

\subsection{Summary of the research}

From the perspective of a practitioner first and a researcher second, I find it striking that the recommended lens for a CAM therapist and a researcher of CAM therapies can be so different; almost as if each were the antithesis of the other. As a therapist, the aim is always to take the various fragments a patient brings and restore wholeness to them, in whatever way able, guided by holistic principles and philosophy. In research, the advised aims seem to be always to take wholeness and fragment it into smaller pieces, guided by reductionist principles and philosophy, for the purpose of enhancing understanding and generating evidence of clinical benefit. This creates a novel research-practice gap. Not in the form that research evidence is failing to be implemented into clinical practice (although this may be happening too), but in 
the form that design, conduct and reporting of studies that serve to generate the growing evidence base often struggles to match the paradigms within which real-world practice operates. This is a dichotomy that I find defies logic, devalues practice, and damages the potential for meaningful clinical benefits.

Perhaps the best, most accurate summary then, I can make of the current state of CAM research, is that it seems to suffer the same research-practice gap that nursing has also seen. Mulhall (2001) says [of nursing] that this is because much of the essential evidence for good practice comes not from theory driven research, but from practitioner-centred research; yet research continues to be enacted and perceived as "an elite, difficult and academic activity" (Mulhall, 2001:391). Progress argues that research should instead be grounded in knowledge gained from experience and that engaging in experience may be a strategy for closing this 'gap'.

It remains important that the emerging evidence base within CAM is generated with quality and rigour, but a lack of congruence and resonance between the paradigms/processes of popular research methodologies and CAM phenomena is failing to make the most of potential research efforts in this area, thus impacting both current policy and future provision. For all the investment there has been (which is nothing like eno ugh) into this area, current trends are only superficially scratching the surface, when they ought to be looking beneath. Indeed, to apply the principles of CAM to the research in this area, would dictate that that any appraisal be multi-dimensional, and loaded with "equal if different value" (Cassidy, 1994:11). Those working in this field have not behaved as congruently with the modalities investigated as they should, and in this respect have replicated the same fragmentary and reductionist approach that in many cases has been implicit in the rising popularity of complementary and alternative healthcare practices for patients.

Both the scale and nature of this rise are currently clearer than ever, as a result of data generated by the recent CAMbrella project (see 2.2). Prevalence data, legislation information and the attitudes and needs of citizens across Europe, have never been more current or accessible (Eardley et al, 2012; Wiesener et al, 2012; Nissen et al, 2012), and provide important contextual dimensions to any understanding of CAM, although perhaps more in terms of quantity rather than quality (there is no in-depth knowledge about how these findings impact the 'local' experience of therapy). Previously, Andrews and Boon (2005) provided similar data from a Canadian perspective, but also abstracted from any specific sample population. 
Addressing this would, as Andrews et al (2004:183) have previously noted, be "a considerable research endeavo ur", which may explain why such work has been slow to develop, particularly at the micro-level where subtleties in the provision and experience of CAM can be best detected. Current funding directives, political agendas and research expertise to cross this divide may also hold some responsibility, as may the limited numbers of researchers interested in developing this field, often spreading their activities across multiple areas of healthcare (Andrews \& Moon, 2005b). Also inhibited by the perception of contextual influence in healing outcomes as largely distanced from the 'serious business' of CAM research, this has in recent years prompted instead studies to establish both prevalence and efficacy for the 'active components' of interventions as counter to the popular criticism of CAM therapists as 'quacks' (Maple, 1992; Wahlberg, 2007) and the ina bility of CAMs to generate efficacy 'beyond placebo' (Kaptchuk et al, 1996). Contributing to this, the House of Lords (2000) have clearly suggested that use of RCTs (Randomised Controlled Trials) should provide the 'gold standard' for building a CAM evidence base, just as within conventional medicine (Ernst et al, 2005).

However, many maintain that much of value (often either from a theoretical or experiential perspective) is controlled or eliminated within such research designs, with practitioners reporting that these methods "dissect their practice in a reductionist manner", thereby failing to take into account the role and value attached to their holistic nature, which leads to invalid evaluations (Mason et al, 2002:832). Calls instead, to return to the concept of 'weight of evidence', are being made whereby multiple forms of evidence are considered together and synthesised, to form a composite opinion of effectiveness (Kaplan et al, 2011; Walach et al, 2006). Not unlike the 'Mad Hatters Tea Party' I witnessed in Berlin, it is possible here too, to go round and round but never forwards, such is the volume, breath and lack of consensus in the material.

In terms of engaging and appraising literature for this study, I have found a variety of approaches which either presented as attempts to be progressive or holistic, those that could or should have done so but failed to try, and others that offered explanations or critiques of the reasons behind this with some suggestions going forwards. Within this, there are also different understandings of the limits and potential of CAM therapies.

\subsection{Developing a question and aims for study}

My brief, within this process, was always to enact a study with the title "Comparing complementary health centres in the UK and Europe that use a combination of therapies". I never thought to question or challenge this (even though, or perhaps because, it was selected 
prior to my appointment) as it already seemed to offer eno ugh freedom and flexibility to make it my own. I even felt, coming already from a broad undergraduate education in CAMs (not just my own practice discipline), that I could offer something 'especially unique' here that many others, with a different background, may not.

This engagement with the literature provides many questions I feel need to be asked, however, upon reflection I feel that many of these relate to a general lack of clarity regarding the use and understanding of many CAM therapies. In particular, there seems little agreement around understanding and application of the term 'CAM' (defined often by 'what it is not', therefore leaving 'what it is' open to interpretation), and no contrasts or comparisons of individual differences on a practice or experiential level. I fear that none of the (current or future) questions being asked by researchers may be answered properly until some clarity is first achieved regarding real-world CAM practice, which appears to lacking in most studies and methodologies. This means that my fundamental aim is to challenge the current paradigms of CAM research and enact a different story.

I had entered this study aligned with a holistic paradigm: a way of thinking that allows, even compels, me to see more than single aspects or what the surface shows. From applying this perspective to the literature, I see that much of CAM research simply 'misses the point' that CAMs originate primarily to treat peo ple, not problems. I have already spoken abo ut the value in understanding CAM spaces (practice and research) as complex places where complex interventions reign. This is my experience and a gap within the literature that may provide some much needed clarity to aid future studies. Rather than unpicking the complexities, however (which some have tried but with limited success), I feel that viewing them together, as part of a whole performance, may provide something 'different' and a challenge to the use of standard clinical trial models, as advocated by Ernst (2010b), for generating valid and reliable evidence in CAM.

There is an irony to this: that CAM evaluations typically centre around their effect on specific symptoms rather than the whole human condition, yet in practice, many CAMs prefer to treat people, not problems. This suggests that much CAM research perpetuates a reductionist perspective of health and illness that may be incongruent with their practice. This almost sets any study up to fail (if these are not the intentions behind treatment) and, while this may serve the 'research agenda', it does not serve the 'practice agenda' which can affect both transferability and trust in research outcomes. 
Freshwater and Rolfe (2001) put this well; first recognising the challenge of research [again primarily in nursing] to devise, test and apply methods that are appropriate to the needs of all stakeholders (including practitioners) before describing how this can be achieved, by leaving "the high, hard ground inhabited by academics and go down into the swamp where practitioners go about their daily work, to enter a milieu where it is inappropriate to follow the formally agreed method of writing up research as a logical, linear process; that is, by writing out the mess and confusion and presenting the research process as objective and uncontaminated by the real world of practice" (Freshwater and Rolfe, 2001:527). Perhaps a controversial assertion, this has congruence with the holistic premise of study, and may also serve to explain the disparity in the qualities of design, execution and any results of studies included in current systematic reviews of CAM therapy that often result in inconclusive findings (the Cochrane Library has many examples).

Actually advocating 'writing in' the mess is an interesting turn that challenges the trend, in recent years, for healthcare research reporting to become more and more sanitised, like its practices. While this may, in theory, focus the reader on the positive or eventual outcomes of the research, the risk of writing such experiences out of the final draft (and so 'end up on the cutting room floor') may also lose something of the overall message, about generating the findings that are presented. My experience as a CAM practitioner, and patient, suggests this is exactly what therapy in the real-world is like - messy - and likely explains why I (as other practitioners) have become disenchanted and disengaged from including research findings within my clinical practice: I simply do not recognise them.

Vallance (2006) describes this as "the murky non-specific noise that muddies our objective understanding of therapeutic processes" (Vallance, 2006:287), however, it is unknown if the backdrop for practices of therapy and practices of research may yet prove key to interpreting these performances. This could be an issue of great importance, as the CAMbrella group suggests current CAM provision in the EU is supplied by approximately 300,000 registered medical and non-medical practitioners and "with a huge variability" (von Ammon et al, 2012:42).

Bringing a holistic lens to a review of the CAM literature has proved both a challenging and illuminating process, and when I began this, I never anticipated it would push me to take on an effective 'reform' of the whole culture of research in CAM - and yet here I am! Created in response to the literature and borne through applying a scaffolding process to my own perspective, the question I now feel prompted (even compelled) to take forward and answer is 
as broad as my title, allowing both 'space' and opportunity for true inquiry, unrestricted by pre-determined aims or outcomes. This presents CAM research like the 'patient' who, after receiving unsuccessful treatment at the hands of a conventional practitioner, turns to alternative approaches seeking a more holistic solution either as a remedy or a catalyst for progress. Therefore, with the hypothesis only that there must be something that CAM research is currently missing - or research would already be better than it is, I ask:

"What can a CAM practitioner's perspective of studying CAM practice teach CAM researchers about researching CAM?"

Thus, my engagement with this study is an attempt to explore the process of enacting holistic research in CAM, and what this can teach me a bout how I view and engage with CAM research culture as a practitioner of therapy, a practitioner of research and a person. My aims then, are to develop a way to do this and learn from the process.

I also hope to pass a 'candle' of enlightenment between myself and the reader, to spark their own curiosity and stimulate their similar exploration into how they engage with their own processes and products - whether clinically or academically. I do not deny that knowledge of the parts may be necessary, but if this comes at the expense of the whole, then research may indeed have 'lost the plot'. 


\section{Chapter 3 - Re-writing the script...}

In order to address this task, I first needed to identify a suitable approach, sensitive enough to capture and value the complexity of therapy, thereby enhancing model validity, but was also robust.

I started by looking at existing qualitative methodologies and methods, hoping to select one to better use or adapt to my unique proposal. Then, during a workshop entitled 'turning ideas into questions' led by one of the professors in my school, I was clearly advised that my type of question could not be achieved within the parameters of current research - it was simply too big to be possible. For a while after this I did consider trying to narrow my scope but found this presented me such inner conflict (not only did I really want to perform holistic research I NEEDED to research holistically, just as I practice therapy holistically and try to live holistically) that in the end I rejected this a dvice and continued on my own path.

I did begin to see, however, that current research approaches did pose some limitations, regarding the extent to which holistic research may be realised. Certainly, survey methods were never going to reveal the full nature or role of complexities within practice, as has been discovered elsewhere (Turner et al, 2007, Harris 2010), neither have interview based studies, as the focus of results typically become centred around the outcomes achieved and impact of relationships in this (Di Blasi and Kleijnen, 2000, Long, 2007; Smith et al, 2009). It is notable too, that in the race from early prevalence and attitudinal surveys to more focussed clinical trials and audits, somehow the 'components' of interventions, rather than therapy as a whole (holistic) event, have become the common study target. I regard this as being due, in some large part, to the ways Evidence Based Medicine has been adopted and applied within the healthcare professions and associated research (Hoover, 2012).

Compelled to 'see' and 'act' differently from the status quo, I was encouraged by my supervisors to connect with, design and execute a study from within my own unique philosophical complexity. The primary purpose was always to see what I could learn or discover from this, with hopefully lessons for researchers of CAM going forwards. Certainly, if I look back at my own journey from my first musings about this to where I sit now, I have travelled a long way. I began by consciously challenging the usual ways of perceiving, engaging and interpreting research endeavour, much as Cassidy (1994) suggests, and this has brought for me new insights. Inspiration has also come from many areas: those around me and those I read, in particular the examples of many practising CAM therapists who every day challenge 
the status quo to apply novel or holistic solutions to the puzzles and problems their patients bring. From my own experience, I also find wondrous results from such endeavours and feel potential benefits to be always worth the risk of trying.

Presented with the opportunity to champion a 'new' research agenda for CAM, I came to also test a new approach for CAM study that fits with my own skills and brings the potential for application across many arenas. This has revealed a further dimension of CAM than I had anticipated, and through this have acted out further potential, than I originally intended.

\subsection{Conceiving the production}

While convinced that it should be possible to achieve a more holistic research production, congruent with the modalities under investigation than is currently presented, it took a little while to work out exactly how this could/would be realised. Matched with supervisors to channel my creative enthusiasm for this task, I began viewing both the research landscape and my opportunity to impact it, from many different avenues. Essentially, I learned that the rules of CAM differ from most people's 'reality'. This is because in society, the biomedical paradigm often dominates not only the ways we interpret illness and access healthcare, but also the ways we typically view and engage with the world around us. The parts of the whole are therefore often disconnected into physical and non-physical, with little real concept of how (even if) the two interact.

CAMs typically view the world as some level of interconnected whole, whereby cause and effect patterns are non-linear and many things beyond the realms of science, are possible. With regards to health and illness, symptoms are seen as a symbol of imbalance, and treatment aims to regain balance and support healing. Evidence of mechanism may, therefore, be less important than evidence of outcomes. Such a perspective is incongruent with academic exercise, yet the biggest challenge I needed to overcome if I was to achieve the aim of conducting research that views therapy in the same way that CAM practitioners and patients do, was to establish a way of interconnecting the acts with the experience of these acts.

I knew that comparing data from different settings could create a unique potential to illuminate practice as Paterson and Britten (2008) found, plus I already had the title of my study prior to gaining my scholarship position (these were tied), so the expectation was always there. From my previous experiences of research (as an undergraduate) and my engagement with the literature reviewed here, I knew I indentified more with interpretivist than positivist 
traditions, and so would naturally favour methodologies and methods that supported this. It was important for me to practice research as authentically as I practice CAM and I was excited to discover how my 'alternative perspective' may resonate with the real-world of therapy, and research.

Looking into my own practice was an 'artistic' exercise and not just a therapeutic one. I learned that, when I worked clinically, I created both the space and a model of practice to suit both the techniques I was trained to deliver and my own personal preferences (where possible). This changed, as I changed through undergraduate study, to reflect more of the holistic ethos and theoretical awareness of different CAM philosophies I now had. This was always reflected both in my manner and how I chose to 'stage' my practice, best supporting the therapeutic potential I wanted to deliver.

A most natural approach for me, I saw a real potential for developing/applying this concept within research, to capture and illuminate the different complexities within the setting and performances of CAM that other research may overlook (and a professor in my school even said was 'simply not possible'). Conceiving this as an idea for a 'research production', I began to wonder if 'staging' environments (for affect?) was something that other practitioners also do, and whether this could provide the way into comparative study of complexity in CAM therapy practice to determine any values associated by practitioners and patients to the ways CAM research is performed in different settings, to better inform current therapy evaluation or appraisal of already published studies.

\subsection{Ethnography as a basis for study}

I based my application of this on Ethnography. Ethnography is a methodology I had used previously (as an undergraduate) and I felt naturally fitting with the aims of my inquiry now. Concerned "with the meaning of actions and events to the people we seek to understand" Spradley (1979:5), this creates the potential to describe in detail the actual living situations of societies and environments, revealing the range of explanatory models used. In addition to observing behaviour, it also questions the meaning of that behaviour and the values assigned to customs, artefacts, knowledge and experiences, which are subject to both geographical and cultural influences (Moerman, 2002).

This seemed to give me the freedom to enter, and engage in the different practices of CAM, and bring back novel insights in a way that I felt other qualitative approaches, such as grounded theory, may not. For a while, I also pursued the idea of incorporating some 
quantitative measures (to triangulate my data), but in the end I felt this would divert my aims from discovering the 'hidden' potential within CAM practice, to benefit CAM research.

Many of the modern ethnographies include developments to incorporate a more discrete, sensitive and creative observation and open up the potential for noting unique or complex matters, by weaving elements of these (setting, props or costume detail) into the narratives Goodall (2000). This gave me confidence to revisit and then pursue the idea of 'staging' as a means of engaging the deeper levels and connections within practice, fitting with my own history of 'staging practice' and something that I felt I could really enjoy doing.

\subsection{Theatre as an adjunct to ethnography}

Pushed, almost, by the critiques of those around me, I began to wonder if there was anything I could do to enhance this approach and ensure a comparable application across diverse sites. Not quite a quantitative measurement, I hit on the idea of 'documenting the staging' of therapy, within a categorised structure, to 'set the scene' for both what I want to achieve and how I want to feel while doing this.

The theatrical metaphor, while not usually applied in clinical care, is well established. It's use is common within the fields of philosophy, psychology, anthropology, ethnology and sociology (Fischer-Lichte, 1995:85), and examples are also evident within Business (Anderson, 2005), Marketing (Fisk \& Grove, 1996; Goodwin, 1996) and Organisational Management (Gardner, 1992; Graham-Hill \& Grimes, 2001; Harvey, 2001; Oswick et al, 2001; Walker \& Monin, 2001; Wood, 2002). Following the examples of Goffman (1959) and Burns (1972), who each use the concepts and terminology in theatre to make the 'unfamiliar social world' accessible to others, I saw potential for using this metaphor here, to provide a consistent means of describing behaviour across the different samples and sites proposed. Indeed, Helman (2001:5) has previously compared spaces such as a doctor's office, hospital ward or holy shrine "to a theatre set, complete with scenery, props, costumes and script".

I began to discuss with my colleagues and supervisors the potential of employing this concept across the different sites and samples in my study, prospectively, to capture and explore the potential for 'meaning-making' within therapeutic encounters. As I saw it, the 'language of theatre' had the ability to perceive and map out the terrain of healthcare settings (through the categories of scenography, a theatre sub-discipline) as easily as any action and experience within the whole event. The key difference here would be of theatre no longer only a 
metaphor, but as a methodology and method for structuring and capturing the nuanced detail within complex social performances (i.e. CAM).

My supervisors were supportive of this idea and my primary supervisor even began to explore if he could use the same metaphor within his practice. We all felt this could be something new and exciting, and together we jumped headfirst into this 'novel' approach - and I into the unknown. Shortly after, I joked with some of my post-graduate colleagues that it seemed not only was I seeking new information with my study but I had somehow also landed myself having to seek a new way to do this. The truth is I did feel I had to do something radical - to make a difference. Whether this was due to my desire to 'be the perfect student' (something which I could only ever aspire to, it turns out) or the pressure of the dominant discourse on my research practice, almost forcing me to reject everything that convention had to offer, I am unclear. At the time, however, I believed that any influence was only pushing me in a positive direction.

\subsection{Metaphor to methodology: the (re)birth of Theatricality?}

Theatricality, a concept within performance theory, has been defined as "the result of a perceptual dynamics linking the onlooker with someone or something that is looked at" (Féral, 2002:105). Refining this slightly, Burns (1972) suggests two possible directions for this to be applied in practice: 1) as a mode of behaviour; or 2) as a mode of perception.

Like Burns (1972), I do not agree that behaviour can be theatrical, only that it can appear theatrical to an onlooker; a position she argues in her seminal text, where she demonstrates how social situations can be perceived and described using the language of theatre. Therefore, the definition I take forward here is of 'theatricality as a mode of perception' which I widen also to include perceiving and describing the composition and role of social settings, as an interconnected and underpinning factor in the acts and experiences.

This raises a real parallel between society as a heuristic and constructed cultural model and 'theatre' also as a heuristic and cultural model, with the "capacity of constructing reality" (Fischer-Lichte, 1995a:89). Viewing one as a reflection of the other, these constructed settings can each be regarded a result of complex components that create a space for performances within where a deeper, nuanced engagement may better facilitate critical reflection on the "process of construction and the conditions underlying it" (Fischer-Lichte, 1995b:104).

With echoes of performance ethnography (Denzin, 2003:12), I find this a good starting point for viewing "performative representations" as a viable means of studying cultural experiences, 
which also answers the call to researchers to create a 'space' where ethnography can be interpreted as performance, situated in a specific discourse and presented/represented in either textual or live formats. Other arts based research methods, for example ethnodrama or ethnotheatre as exemplified most notably by Saldana (2011), also offer the means of presenting and engaging research findings as theatre performances, either in lit erary of live form, but neither of these currently bring together their performances or representations with the spaces or contexts that create them - which is where I see a place for Theatricality as a research methodology.

Changing both the power and potential of this work, I believe, offers the potential to enrich the lens and quality of data capture in a more holistic way and allow actor and audience to cocreate a whole story that plays out differently each time. True, the human condition is central in all of these approaches, reflecting "the humanity of what was provided [and] the personal experience of the everyday lives" within (Robinson, 2010:108), but the key difference for me is develo ping the use of theatre as 'a way in' rather than 'a way out'.

Therefore I propose 'theatricality' as an adjunct to ethnography and a complementary means of underpinning the capture of data from the start, which may or may not be later presented or performed as ethnodrama or ethnotheatre (later meaning post submission of my formal report, not later within this report). Applying the theatrical metaphor as holistic research methodology in CAM brings a potentially fresh perspective on these whole, global events and even creates the opportunity for "doing CAM" just as Glass (2012) talks of "doing scene"; going beyond observing the practices and effects of CAM interventions to seek understanding of the factors that underpin how space, identity and power contribute to and manifest within these acts and the whole context of the performance.

Employing performance theory, in the study of context, already has some pedigree having previously showed a fruitful perspective for illustrating the "embodied sense of being in and experiencing the uniqueness of place" (Rogers 2012:65). Here, I was placing myself in a growing overlap between geography and theatre, to view social worlds and behaviours within as performances, and from this revealing a potential for implicit natures of place, power and relationships to be dramatised, as cause and effect and with influence for the roles of all. But as I have travelled beyond this, so does now my research and I do not confine my definition or perception of space to the physical or even 'dynamic' alone, between practitioner and place or patient and place, but also to the intellectual between researcher and concept or researcher 
and data. 'Space' then can relate to the physical spaces of therapy events; the emotional spaces of human events; and the intellectual spaces of research events.

Blending the traditional approach of ethnography with the novel approach proposed of theatricality, was a transformative act from first conception to final execution. On a personal level, I was finally validated to be who I wanted to be (always was on the inside), and actually, couldn't believe my luck in being able to devise a research study that combined my two greatest passions in life - theatre and CAM. This sent me on a high that lasted throughout the whole enactment of the study, and beyond, with the one major negative that it became almost impossible for anyone to 'bring me down'.

\subsection{Setting the scene for novel research}

Including all these aspects in a single study design has, by default, become the challenge I now take on; but then holism is nothing if it doesn't at least try to engage and encompass the totality of all things. I had learned from my engagement with the literature that there were currently no CAM researchers attempting what I regarded as 'holistic research' (i.e. where the totality of the event is honoured or valued 'as a whole') despite vitalistic theory which underpins many CAM modalities suggesting this is how they are performed in practice. I was disturbed by this incongruence as I know from my clinical work that a 'totality view' often yields a better outcome than a narrow one. Therefore I have no option but to try applying my 'holistic view' to see what impact this may create in respect to either study design or outcomes evaluation. In this respect, I am no longer only testing holism, I am also testing myself.

I did not consider, before embarking upon this research, that I would need to script a novel methodology and method to help me achieve my aims. I had felt that existing methods would be sufficient, and these just needed applying within a holistic frame. This frame felt that it was possible to engage with the deeper connections in CAM practice by exercising a more subjective, participatory performance of research that current CAM researchers seemed to enact and so this is where I intended to position myself.

However, as time passed I began to see the distinction between conventional approaches and creative ones, becoming more excited by the potential of the latter, particularly as I came to identify healing arts as first and foremost arts that can neither be adequately acted or examined without involvement and engagement of the spirit. There was no 'off-the-peg' script for this nature of work that seemed to offer what I needed, so I sought to identify a suitable genre (methodology and method) to tell my holistic story and develop this into a coherent, 
applied research approach. I also wanted any method, as much as possible, to be capable of consistent levels of sensitivity in capturing everything (both familiar and unfamiliar) within the various complex environments that may have use or value for the participants, and not only be something I could enact; yet also positively utilise my own experiences as a therapist.

According to Myers (2010:1301), setting the 'scene' for therapy is perhaps the most embodied and yet overlooked part of clinical productions, relating much within 'healing' to 'the performance'. Congruent with theatre semiotics, it is further considered that the active backdrop that supports and enables all presentations and performances can be considered worthy of capture and exposition, evidencing the underlying discourse of the place and/or those who work there. Containing both cues and clues to the intent and expectations of any characters, and influencing multiple aspects of the whole production including therapy experience "the design contribution may be limited to the functionalistic or merely decorative. Conversely, it may be integral to the realization of the text, counterpointing theme and ideology, image and symbol, in pictorial terms." (Aston and Savona, 1991:144).

Within my own clinical practice, I have typically introduced softer lighting than was institutionally provided, may have also used lightly fragranced candles and even played soothing music in the background for the primary functions and specific effects/affects of amenity for me and comfort/relaxation for my patients. Certainly working in different environments (patients/clients homes, a private gymnasium, Turkish baths, my own home, a primary care facility and finally a sole commercial let) has always impacted me and the performance/quality of care I delivered there, even if I didn't always fully understand why. I do, however, know that I never consulted with my patients prior to creating each setting, or particularly sought their response to the environment where we connected in therapy. This surely says something about me or my practice!

\subsection{The importance of being characters}

Outside the setting, theatre semiotics also suggests that characters are the central aspects to any story with simultaneous roles which they perform onstage, offstage and backstage. Each one of these is a whole, complex and unique component that breathes life into the plot and proves pivotal in mediating the dramas they create and experiences they share within the setting. Sometimes cast in their roles by personal appointment, or management decision, even social and political enablement characters equally influence and are influenced here. 
Aston and Savona (1991:46) state that "within the theatrical context, the actor serves as the agent whereby character is mediated to the spectator". This may carry beyond the theatre and into social worlds too, which means that in therapy, knowing who these unique and complex individuals are may be vital to understanding their whole experiences or their drama and the impact they create for others. I feel this may be particularly so for practitioners (if taking the argument about influence of discourse) and to understand the response that occurs when these individuals mediate or negotiate their performance to account for local or contextual influences. This has certainly been shown previously within trials (Kyle \& MarksMaran, 2008; Paterson \& Britten, 2008), limiting both engagement and interpretations of the whole scene/resulting data to a superficial level and leaving gaps in our knowledge.

Bringing both richness in texture and colour to each event, characters can display elements of their unique journeys, their personal biographies, their relationships, perspectives and aspirations in the presentation of the drama to anyone with a mind to look and to see this. From their motivations to their idiosyncrasies, whether playing major roles or minor ones, uncovering these stories (through observation, reflection and interview) can provide a unique opportunity to add depth and meaning to any scene, so value and role may be adequately understood. In addition, through these characters, it is possible to explore the extent of influence each has over their own contextual details (critical for a fully holistic appraisal to be possible). 'Knowing' these characters, therefore, on a personal and human level is a goal, with the reward being connections within the data, which may not otherwise become uncovered.

\subsection{Finding the plot}

Within each story, the elements of past, present and future means that data is never limited to only the immediate observations, but rather a series of single events or episodes within a much larger story. Providing then "the means by which narrative events are structured, organised and presented" (Aston and Savonna, 1991:21) the plot, whether constructed or improvised, will direct the action and helps each story to flow, to its natural conclusion. With always a 'shape' to these events, they can then be observed and compared between productions/sites. Typical aspects within therapeutic encounters may be: the purpose of the visit; a detailed account of the interactions or interventions that are applied; interpretation of both spoken and unspoken dialogue; and any responses that are achieved from these within each individual scene. That is the plot of therapy.

The plot of research is also an active one, within the story, complete with any number of challenges that must first be faced and then overcome. Uncommon to include, these days, this 
means recording the role and impact of research failures alongside any successes (they are all important to the final story) so the overall outcomes and results may be contextualised. This can, at times, be hard to connect or find a way into, particularly when pre-occupied with other aspects. However, where awareness is achieved and demonstrated, a further layer of data can be added, giving more depth and potential relationships within this. This also challenges any presentations as sanitised, bland and generalised and demonstrates the power and value in the plots outside the immediate characters and setting (including me), suggesting why these must also (where possible) be considered and woven into the final story.

\subsection{Creativity as a research process}

Arriving at a 'novel' coherence, I feel to have developed a credible potential for enacting holistic capture of CAM practice data. This has been a personal trial, as I have faced a barrage of advice from many different directions (training workshops, discussions with school professors and written texts by high profile academics to name but a few) that my holistic aims "could not be achieved within a PhD study" (personal communication) and I should instead select an aspect or component of practice to explore. I had come into this process believing that I was in a position of some support, that within the walls of academia there would be people around me to help me 'find a way' to achieve my aims. The wholesale attitude of proposing reduction be adopted instead (the antithesis of CAM, vitalism and holistic medicine) instead created a situation where I began to feel more and more isolated.

Evident beyond this, too, my Father asked me recently, "Why has this study taken so much out of you? Couldn't you just do things the way everyone else does?" I answered, "I wish I could, but then what would be the point of that, if the ways others are currently engaging in CAM research means they are struggling with so me of these issues too?"

What I meant by this is that I do not see complexity, heterogeneity and different philosophies or paradigms being adequately supported by RCTs or other popular methodologies - because these CAMs are not standard. Isn't it an example of insanity to repeat same behaviours and expect a different outcome?

I couldn't simply maintain the status quo if I felt this was inappropriate. Like drowning in an intellectual whirlpool of research paradigms, I was desperately gasping for a solution, permanently preoccupied with philosophical debates regarding "how reductionist and holistic philosophies can integrate, either within academic or thera peutic arguments?" and "whether a study of holistic therapy in a reductionist, fragmentary way betrays the very essence of the 
modalities it aims to evaluate?" - debates that no doubt many of my colleagues, both within the university and whom I have come to know through external conferences and research groups, have also faced.

'Engaging creativity' actually saved me, which is why I now view this as part of my whole methodological approach; this has not simply been an excuse to bring an artistic/theatrical approach to serious study (which has been fun, but also extremely challenging). Then, as now, I believed that holistic research should be possible (even essential), within a study of holistic therapies.

The concept of theatre as a global experience, where every aspect is a vital part of the whole event (Duggan \& Grainger, 1997), offers a unique parallel to the performance of CAM therapies, which is why I developed, trialled and evaluated this, within this study of real-world application and experience. This road has been a difficult one to travel, particularly as engaging within a 'war of paradigms, discourse and methodologies' has caused me to become increasingly distant, even isolated, from many around me (including, at times, my supervisors). It is ironic that someone conducting a study about holism and interconnectedness should feel disconnected while doing it! But then I have learned, through my engagement with the many different characters along the way that my originating (western) definition of holism as a single mind/body/spirit entity, in underpinning myself and my study, may have contributed to this inability to connect with those others as well as I had hoped. But then every new discovery begins with one person's idea being tested and for every success there are likely many, many failures first.

Through positioning the genre of theatre as a mode of healthcare study, to capture and tell the story of practice within an original script, I hope to aid both future study design and evaluation of CAM therapy. Well suited to a study of unique and complex real life situations, I find theatre outperforms other dramatic mediums, as scenes once performed here cannot be sanitised, edited or re-run. This displays a distinct difference from other forms of expression.

\subsection{Performing reflexivity}

Perceived also through the lens of theatricality my whole act and actions within this study, from the development of a novel methodology to the final written performance, can be viewed a dramatisation of Moerman's (2002:99) argument that the determinants and the freedoms of composers are, like our own unique stories, influenced by the unique cultural context of location, place and time. 
I truly had no idea what I expected to find when I entered, and ultimately returned from, the field, except that my reporting would not be limited to the practical application of treatment protocols, even if that was how therapists applied their care. What I did know, was there had to be something about real-world therapy practice that research was failing to capture or value, or how else could clinical research reports and patient anecdotal or case study reports so consistently suffer from disparity in findings? If it was not going to be this, it would have to be something else, but at least I would have taken a look!

An unexpected finding from this was that, while inside this 'process', I had no ability to access (external) abstract and theoretical concepts; the result of which was to leave me without the ability to critique or contextualise my subjective feelings and thoughts, within this large whole. This raised for me the question about the ability for those on the outside to really see or connect with parties on the inside of phenomena. I was on the inside here, and texts written by outsiders had no impact on me once I was no longer on the outside too. 'Feeling' replaced 'thinking' and I had to learn a new and different level of subjective reflexive engagement to 'get out'. Here, an unexpectedly critical take on the concept of performative criticism (I will explicate this more in the next chapter), demonstrated that coherence in design really can enhance the ability to connect the parts. This 'got me out'.

As a holistic thinker, this thesis model has provided a way for me to engage a holistic frame within research study; from conception of ideas, through appraising the literature, selecting methodologies, methods and design to ultimate execution, analysis and synthesis.

At times, and despite my conviction, this was so very hard to enact. However, I feel now, that what I have sought to produce represents a radical challenge to, and critique of, current research culture with aims that are pure, open and honest. I did not alrea dy know the answers or where this research would take me, when I started on this journey, but I was excited to go there and I am happy with where I have arrived. 


\section{Chapter 4 -Scene as a Whole (Methods I)}

As noted, what I came to term 'Theatricality' is different from other arts based or dramaturgical research approaches, which typically focus on recording and noting behaviours in 'space', with attribution of outcomes related to either the characters or plot (fitting the four key dramaturgical concepts of comedy, tragedy, melodrama and farce). What I was proposing was instead to take the scenographic categories of scenery, set-design, lighting, properties, costumes, make-up and sound, to record both the composition of these within holistic performances and how characters engage or take/make meaning from this composition, and then interview characters to gain further clarity on what I had witnessed.

This approach to combining therapeutic practice and experience 'as a whole' is located within a novel study methodology where I, as 'an actor', have mediated the performances within. In this single arena, I openly acknowledged these experiences as whole/global events, which proved a highly suitable means for generating data in complex therapeutic performances. I began by selecting what I felt was a valuable research question, to fill the gap I perceived in the literature and then developed a new methodology and method to answer this question with authenticity. This contributed to the building of a complete picture of therapy as it is 'staged', 'performed' and 'experienced' with the factors that underpin these, and any influence or affects on participants, illuminated in therapeutic 'performance ethnographies' (Smith \& Gallo, 2007).

An essentially heuristic approach, my development has occurred alongside rather than with, or even after, other researchers with the same aims, although my writing up has been able to benefit from and reflect on the progress and results of other research teams who completed their current tasks before me. Having no study guide but myself and no critical frien ds but my supervisors has meant my learning journey has at times been a very lonely one. That said, I steered what often felt like a clear path through the design and execution phases of the study (I suppose familiar territory given my previous experiences in staging productions) finding the next steps, of objectively synthesising and writing up my findings in a coherent way (coherent to anyone outside myself at least), to be the most challenging.

\subsection{Suspension of disbelief}

A key component of enacting this approach 'in the field' is the theatrical convention of 'suspension of disbelief'. This requires entering the performance as an actor, willing to engage 
in the realities of this created world on its own terms (these can be dissected later through critical review). Whilst in this world, everything is real and everything matters to someone. It cannot be disentangled or the whole thing starts to fall apart, changing into something else entirely (a different whole event, if you like) affecting both engagement and enjoyment - it is much harder to cry at the end of 'Miss Saigon' if you keep telling yourself they are only actors and none of this is real!

Indeed, part of the critique, after such a performance, is how well the actors inhabited their characters and moved or convinced you of their stories. From the outset, this is a component that I feel current research approaches overlook and something I want to avoid doing here (as a variable, I believe we should engage more, not less). Stepping into therapy productions and starting to unpick these events 'during the performance', as in trials, is distorting care (Kyle \& Marks-Maran, 2008; Paterson \& Britten, 2008) which affects the (research) audience's whole engagement with it. My insights and experience as a CAM practitioner already told me there are aspects of my therapy performance that matter to me yet the 'critical distance' of a researcher would likely miss. Tapping into these is what 'suspending disbelief' is all about.

It could be argued that such a subjective engagement threatens the validity of the data generated and its critical appraisal. I accept this threat, but suggest that this is only the case where a researcher fails to re-engage their critical disbelief on exiting the performance and remain in a subjective or 'spellbound' engagement. I know this, because this is what I did initially - I became so affected by my experiences that it proved be a real challenge to move beyond these, 're-engage my disbelief' and achieve the kind of critical distance necessary to both analyse and communicate my findings. This is an ironic mirroring of the inability noted of researchers already established in the biomedical model of research, to move beyond their experiences (Ritenbaugh et al, 2010), that I learned could also happen to me.

Having critical friends (my supervisors) to flag up when my appraisal sounded like it had failed in this and was appearing too uncritical, has proved a vital measure. Indeed, my primary supervisor, at one point, accused me of 'converting to the cult' as I had become so embedded inside the data. He further suggested (only weeks prior to my re-submission), this proved the "methodology was flawed" if it had dragged me in so deep that I lost my critical ability and perhaps we "had all become so 'caught up in the gimmick' that we hadn't seen it sooner" (personal communication)? I did seriously consider this, but as I had found this approach to be incredibly successful in terms of capturing incredibly complex practice data and revealing hidden insights of experience, I decided I could not accept that the methodology was flawed - 
rather the flaw laid in the way I had neglected to fully re-engage and communicate this 'reengagement with disbelief', through my writing of it. Instead, I had become caught up in the 'revelations' that it was just not clear enough, when I was speaking from inside the data and when I was speaking from outside the data. It was, therefore, also a clear oversight not to specifically articulate my use of 'suspension of disbelief' in this, before now!

\subsection{Engaging the action}

Participant-observation, a method for engaging with the stories of research participants, seemed an obvious choice for an ethnographic-based study, and equally applicable to the concept of 'audience in the theatre'. Placing the researcher into the production in this way gives a voice to the various gaps, left by other researchers.

Engaging as theatre, I found data collection could become both a conscious and unconscious act, where I would have a role in either the audience or the action (active to and critical of the whole performance, at the same time). Congruent with the study methodology, through which social settings (and the performances within them) are perceived and described in the language of theatre, this also acknowledges and utilises the 'cumulative meaning making' that researchers can encounter when in the field, and feel compelled to deny upon their return as they enter a more objective appraisal of the data.

If I dramatise this, as a visit to the theatre:

Arriving, my gaze first takes in my immediate surroundings, the entrance, the foyer, signs that will direct me to the various areas of the building (restrooms, bar area and most importantly my seat), and the bustle of patrons and staff also trying to navigate this space, fulfilling their various roles. Interrupted by the actions of those around me, I find my seat, remove my coat, and turn my gaze turn to the splendour or simplicity of the auditorium. My focus remains on the physical environment, the construction and design all affecting me, creating my anticipation of this event, until I become settled, acclimatise to the surroundings, and relax enough into them to enter the world lying beyond the safety curtain. (My dialogue)

This approach contains an active-reflexive opportunity to participate in observation with synthesis, in a holistic appreciation of the acts, as they are witnessed. Therefore, to be holistically faithful to the phenomenon of whole therapy practise, I intend to first open myself to it, not only observe and talk to people, but to gain a personal contextual understanding of their experience and realities alongside my own. Achieved by immersion within the 
constructed nature and fabric of therapeutic field settings, this provides a thorough foundation and engagement with the spatial context, the actions that occur within the space and the dialogue/stories of other patrons and actors. Such 'intimate knowledge' is, as Goffman (1989:125) argues, essential in order to "penetrate [participants] circle of response".

Appreciating not only what is seen, but also not seen, demonstrates a breadth and depth of multi-level and multi-dimensional engagement with events that is congruent with my holistic paradigm, and authentic within the selected methodology. Ethnographic field notes, alongside interview transcripts, also have the ability to capture and record various dimensions of complex experiences. In practice, however, these methods with their focus either/both on what people say or what they do, can fail to holistically 'connect' the impact of specific details with the very 'space' of the physical and dyna mic setting.

I continue:

Immersed now, in this space, the unfamiliar environment recedes into the background, temporarily. When the curtain rises, my focus shifts to the staging and set, onto which the actors will enter and begin their performance. Clues here enhance my ability to connect with the location and context of the story, the lighting tells me about the time of day, and the costumes and props all locate the place and time in history, long before anyone speaks. When the actors do appear, I meet the characters they portray and follow them on their journey, sharing their story as they relate to each other, and to us. Through this I mould a subjective synthesis of the various parts of the event, into a coherent and meaningful whole. (My dialogue)

A natural part of theatre performances, this 'interconnection' is the pattern I wish to replicate in research study. An engaging technique on every level, active use of self places the researcher in the role of a participatory-audience member, where both capture and interpretation of performance data can be holistically managed. Every aspect of the carefully constructed environment is placed, utilised and recognised for its integral role and value to the whole production, creating a heuristic act that draws equally upon expertise and experiences of 'theatre' and 'therapy' in a multidimensional appreciation. This approach provides a new and creative way of gaining interpretive, contextual understanding and of making tacit meanings explicit.

Containing the power to perceive social productions as theatre, the next step was to transform this process into a flexible research method, capable of ensuring data capture to include 
everything and exclude nothing', with comparability between contexts (this study engages with multiple environments, in multiple countries).

This provides the potential to illustrate the phenomenon from the perspective of the various participating actors and the reflexive-responsive audience in holistic representation. Like my visit to the theatre, such a multi-dimensional engagement with the complexity of the scene, has not been previously achieved within a CAM research context.

\subsection{Theatricality Framework Method (TFM)}

Use of my novel methodology also influenced the design of a novel data collection tool (based on scenographic categories) that realises potential for anything and everything to be perceived and described using the language of theatre, where the observer is equipped to do so, that is they are able to recognise certain patterns and sequences and has an awareness of what drama is, they can describe these in the language of theatre (B urns, 1972).

Within this study, drama is identified as the quality of experience that results from the unique and holistic 'interaction' between characters, plot and set. In this interplay between personal, cultural and contextual influences, both within and even beyond CAM, a whole, alternate world opens up that other approaches using different perspectives may overlook; something that Rossiter et al (2008) have previously alluded to, although more in relation to data transfer and a nalysis, rather than data capture - which is the next natural step.

For my purposes, a simple yet focussed research schedule provided the basis for capture, of both the richly complex and yet deeply comparable data in the various healthcare settings. The primary aim was to see whatever was there to be seen, rather than what I wanted to see.

Table 4.1: TFM Observation Schedule

\begin{tabular}{l|l} 
SCENERY & $\begin{array}{l}\text { Detailed description of the therapeutic (internal/clinical/micro) and } \\
\text { physical (external/social/macro) environments, noting the location of the } \\
\text { centre, the arrangement and layout of the various rooms, décor, etc. } \\
\text { SET-DESIGN }\end{array}$ \\
Identify the access/accessibility of and within the centre, describe any \\
permanent objects, furniture, pictures, entrances/exits etc. \\
PIGHTING
\end{tabular}$\quad \begin{aligned} & \text { Note the types, degrees and changes of lighting used in the various } \\
& \text { locations. } \\
& \text { (PROPS) } \\
& \text { COSTUMES }\end{aligned}$




\begin{tabular}{|l|l|}
\hline MAKE-UP & $\begin{array}{l}\text { Describe the use of make-up/hair styling by participants, and any practical } \\
\text { implications of these. }\end{array}$ \\
ACTORS & $\begin{array}{l}\text { Note the inherent noise and/or specific application of background music } \\
\text { present in the environments. } \\
\text { Note the presence and particulars of practitioners, patients and } \\
\text { accompanying persons, other staff (disciplines/training/emotions etc.). } \\
\text { Document the various roles and activities of those present, including step } \\
\text { by step description of specific acts undertaken by any actors (unfolding of } \\
\text { the plot) from entrance to exit including use and relationship within the } \\
\text { space/setting e.g. arriving/departing, consultations, treatment, staff } \\
\text { meetings, appointment making etc. }\end{array}$ \\
\hline
\end{tabular}

Employing a 'structure' for viewing therapy spaces, like theatre sets, is quite a positivist approach, however, due to pressure already mentioned I felt utilising this would lend my otherwise fluid study the substance that others around me suggested it needed. In practice, this did prove a useful 'anchor' in the field that was applicable across a wide range of diverse settings and cultures. Wherever they are created, performance spaces are composed of scenographic elements (i.e. scenery, set-design, lighting, properties, costumes, make-up, and sound). This natural scenographic framework, when used to document complex spatial composition, reveals a holistic and dynamic complexity that impacts both the characters, and the unfolding plot (which is why theatre directors take such care when 'staging' their productions). Taking the view that healthcare environments are just as carefully staged, detailed documentation of the scenographic elements brings the potential to reveal much that is routinely overlooked, and even provides a route for researchers into a level of immersion close to that of their 'creator'.

Guided by this TFM observation schedule, each space (external, communal and therapeutic) was viewed from the perspective of an audience member and described as if it were a carefully constructed theatre set. Set plans (sketches) and notes were made together in a fieldwork journal, under the relevant scenographic heading, detailing the choice, composition and placement of materials and objects, and the relative impact of these within the whole scenic composition. The thickness of these descriptions meant they often ran into several pages. I often described elements of the setting that some would consider superfluous, particularly in regard to therapeutic application, as I did not want to be the one who decided what was important and what was not. I was also careful never to pre-judge what aspects of the spatial context would or would not have active purpose and view the 'scene as a whole'. 
Embedding my engagement with these spatial dynamics to a level comparable with that of the creator or architect of the space, I achieved a level of familiarisation often beyond that expressed by many participants, for whom such relationships were largely tacit and explicit. Active in the art of documenting the various field sites, I felt a duty to ensure that each description would provide sufficient detail of the 'space' that they could (in theory) be recreated or re-enacted in a nother location (for example, in a theatre production 'on tour'). This brought an added benefit of making stage directions simpler to create and, in my later action observations and participant interviews, preparing me to adopt the role of informed interviewer, able to better empathise and therefore engage any commentary made.

I found, once engage and stored in my imagination, these descriptions were also an unstoppable asset, invading my cognition and re-creating a live performance 'in my head' each time I worked with an interview or reflexive note (which further enabled holistic and dynamic comparisons to be made). Supported also by the collection and use of photographic images of the different spaces, these perceptions remain firm and almost as fresh over time as they did in the moment of capture.

\subsection{Subjective 'spectator' observation}

An integral part of the whole performance, I applied subjective observations of action within the space, wherever possible. Giving 'life' to the spatial descriptions captured using the TFM schedule, while also informing subsequent participant interviews, data from these provided the potential to 'triangulate' data from all sources (the people/characters, purpose/plot and place/set) together.

Formal observations typically occurred after I had first fully described, documented and appraised the individual setting. Co-operation with the practitioners was an essential aspect of this, and all observations were structured around existing daily practice schedules to cause as little disruption to everyday working as possible. However, this served more as a guide than a set of rigid instructions and the unique situations in each centre (sometimes on each day) always dictated how well any protocol could be adhered to.

Completed while performances were in progress, these were active, not retrospective, and captured in the form of detailed stage directions: noting entrances and exits, use of props, dialogues etc. Occasionally, I added reflexive/side comments, after the scenes had concluded, which also became part of the whole data set. 
For treatment observations, I sat quietly within therapy spaces and witnessed the specific nature of action and interactions before me; general observations were similarly conducted but within communal spaces and often with a larger cast of characters present (drawing me sometimes into their dialogue). Aspects noted here, where they also related to observations within the treatment area, were used to compare or contrast the data. In the absence of therapy observations, these also supported and contextualised the interview data and character monologues, to present a more rounded picture.

During this data collection, the primary focus of attention was the practices (actions), with my own responses to these often only becoming clearer or more actively engaged during relistening to the audio-recorded interviews or reflexive analyses.

\subsection{Participant interviews}

As a further dimension to the data, I carried out semi-structured in-depth interviews, with both practitioners and patients, across all sites. Under the concept of 'whole therapy experience', these explored the wider contextual experiences of therapy; from choice, style and use of the general and local settings (including both physical and dynamic factors) and the motivations or journeys into therapy of those involved (patients, clients, practitioners, therapists). Two distinct yet similar schedules of questions guided these: one for patients (appendix 2) and the other for practitioners (appendix 1).

The patient interviews lasted a maximum of 30 minutes while practitioner interviews lasted no more than 60 minutes. The actual timings varied across the sample with participants for whom English was not their first language often taking longer to articulate their meanings, and even employing other communication techniques such as metaphors or mime, to ensure I had a clear understanding of their intentions. Flexibly applied, participants first volunteered the information they felt it was most important/they wanted to share; I only brought them back where necessary and if I felt specific items or issues that I had captured or otherwise required clarification about were not addressed in initial responses. I felt it was important to fill any gaps and ensure my findings could be comparable across different individuals and the different settings. Each interview concluded with an opportunity for participants to share anything they felt it was important to share that had not been previously covered, which proved an incredible source of 'gems'; with some inclusions enriching the data beyond my expectations.

The volume and depth of personal perspectives this revealed, about the role and value of healthcare settings to the whole experience of therapy, uncovered a clear sense of how 
individual contexts influence both the experiences of delivering and receiving therapy. I actually encountered too many characters to include them all individually but I did listen to and value every voice that wanted participate; eventually selecting and showcasing those that exemplified either the most common, unique or compelling stories.

These lived experiences contained a power that my written words have consistently struggled to convey. I had what seemed like endless discussions with my supervisors, before and after my first submissions, about the blandness of my descriptions when compared to their lived affect (exemplified whenever I 'spoke' passionately about these). This has been a constant source of mutual frustration. However, the challenge to retain the vitality of original words is not new. Steiner (2007:3) previously advised that his words be "read aloud and listened to" in order to fully convey the power behind the words, in his Four Mystery Plays, where the struggles to apply the spiritual, intuitive and subjective 'knowledge' to everyday relationships is explored. Similarly, I have now chosen to use full character monologues, similar to the vignettes applied by Paterson and Britten (2008) in their study of holism in acupuncture, rather than interview excerpts within my data 'text' - so the reader may 'read aloud' the words and intent of participants here, and create an appropriate space to better engage with the power of 'their' words.

Written without reduction, these exemplify both the range and depth of participants' engagement, which varied dramatically across the different sites, and also the problems, potential and pitfalls that can be revealed when researchers create the 'space' for this. It was difficult to accept the final thesis would not feature all participants, as I felt such a duty and gratitude to them for sharing their time and stories with me (limits of the piece and too many voices 'drowning' out the message). This is both in conflict with my natural holistic practitioner perspective (which knows that every patient, every problem and every situation is unique) and yet also in congruence with the same (as this allows these individual and unique stories to be included without fragmentation or artificial reduction such as coding).

I have sought instead, to serve those omitted from this final draft, by using their data to support the choices and analyses I made. I may also seek other outlets for these contributions, down the line. Certainly, I am clear that my whole engagement with therapy here would have been less rich and more 'sketchy' had I restricted the number who participated and only selected 'key informants' from the outset. Moreover, the different rates and willingness of participants, across the whole sample, contain findings also of interest. 


\subsection{Enacting the fieldwork}

Prior to commencing any fieldwork, I held discussions with the lead participant at each site (usually clinic manager/senior practitioner), to develop both a working protocol for each situation and ensure I could capture all of the data I sought in the available time-frame(s). Details that informed this included how many rooms each centre had; how many practitioners worked there; what therapies were practised by whom and on what days; and the volume of patients seen in a typical week. The aim during each period of fieldwork was to first meet with the participating practitioners and view their workspaces, agree a time when their spaces could be available for me to document, and finally conduct action observations and interviews. Where possible, I conducted interviews in the spaces where participants either delivered or received their therapy. Where this was not possible, an alternate space within each centre was allocated to me and I played photographic images of the relevant therapeutic spaces to participants during the course of the interview (as visual prompt), in the form of a rolling slideshow on my laptop computer.

The fieldwork was conducted in four phases during 2011. Phase one was conducted in a single-site UK clinic between $14^{\text {th }}$ March and $8^{\text {th }}$ April, phase two was in a split-site Holland clinic between $18^{\text {th }}$ April and $13^{\text {th }}$ May, phase three was in a single-site Cyprus clinic between $18^{\text {th }}$ July and $5^{\text {th }}$ August and phase four was in two separate Denmark clinics between $25^{\text {th }}$ August and $16^{\text {th }}$ September. During each phase I attended the clinical sites on each weekday, exploring the wider social aspects of each location on the weekends. My living arrangements for each phase (primarily selected for their easy travel to the clinic sites) played a crucial role in both framing and supporting my own performance and providing a mindful participation in the wider social context of the participants, which ultimately enabled better dialogue and engagement with these characters and their stories (e.g. their experiences of the local setting and travel modes to attend the clinics with my experiences of the local setting and travel). While resident at each location I purposely 'did as the locals do', with modes of transport used in UK and Cyprus being a private or rented car, in Holland a bicycle and in Denmark either bicycle or bus.

Revealing an unexpected impact on me and my work, each 'home' setting exerted dynamic influences on my experiences of this research also, much like the clinical settings had for participants. This phenomenon, which became more apparent to me as I travelled both through the study and across European locations, became integral to my 'method acting', as I developed a deeper awareness of the interconnected nature of 'experience'. Such reflexive 
thoughts were noted in a series of field notebooks, along with mindful responses to the setting, activities observed and participated in, informal conversations, general experiences and the use of the novel metho dology and method.

It was an initial identified goal, when I originated this study, that I would actively embrace my own sensitivities as both a therapy and theatre practitioner in my use of any tools I developed or adopted. This reflects the observations of Smith and Gallo (2007: 523), that "the audience is responsible for interpreting the performance", and so it follows that the quality of data collected in observational and interview studies can only ever be as good as the researcher who collects it (Burns, 1972; Fischer-Lichte, 1995b). This is a view I concur with and is why I have sought, within this thesis, to be as explicit as possible about the qualities I both brought to, and acquired throughout this journey. However, it is an important note that, among the volumes of data collected, not all of these made it onto the final pages.

\subsection{Performative criticism - a holistic method of data analysis}

When I began planning my analysis, I initially felt I would adopt the ethnographic method put forward by Spradley (1980), as this study is based upon ethnographic principles. However, as the data started to come in and my supervisors began to sense from me that there was 'more to this' than either of us first thought, they encouraged me to think about more creative ways to engage with the data and reveal deeper aspects to the story.

For a while I didn't know where to take this advice, but my supervisors were clear, and I so trusted their advice that rigour could be maintained (even through a novel method of analysis) if there was congruence in all parts of the research performance. Having already begun to capture therapy as theatre, I started to explore the idea of critiquing therapy as theatre.

I eventually stumbled across "Performative Criticism", a term first suggested by Toporisic (2011:1) to identify a potential new method of heuristic analysis (Moustakas, 1990), that embraces whole experience as a "unique spatio-temporal event" and "performance as a kind of cultural participation" (Toporisic, 2011:1). This carried the potential to be repositioned as a form of critical inquiry and "become specialized in the area of analysis of cultural and artistic phenomena" (Toporisic, 2011:1). Essentially, this requires the researcher to critique events from within, as a co-actor or participant, instead of an outsider or 'foreign afar'. I found adopting this approach gave me the permission to widen my 'space' for relating to and with the data I had collected in a more real and subjective way than my previous understanding of either reflexivity or reflective practice and enter into the data. 
A currently un-validated method, my success will be demonstrated in achieving the study aims. However, Freshwater and Avis (2004) have asserted that it is "the quality of the critical reflection on that evidence, and the creative attempts to weave beliefs based on that evidence into the totality of beliefs held by the rest of the community" (Freshwater and Avis, 2004: 6) that afford the criterion for validity, not the means alone of the evidence production. So this gives me some hope. I have certainly sought to be creative throughout this process and meet the challenges set by the preceding literature, the heterogeneity of the field and my supervisors' expectations for me.

Steiner (1898) stated that "an unproductive head will never be able to say something about a productive one" (Steiner, 1898:69), and this says something about the intellectual space I have inhabited (at times) here. Relating also to Read's (2005) assertion of 'space' creating the potential for human relationships, it is notable that when I felt most supported, I was most productive and whenever I felt unsupported, I was unproductive.

Indeed, the sheer volume of rich data I have collected has required a creative approach (in both the handling and interpretation) to do justice to the practices observed and the participants who shared. In essence, this meant holding "dialogue with the data" (Freshwater and Avis, 2004:9). In practice, this meant listening and perceiving the recorded interviews as 'live performance' pieces, reflecting on each as components of the unique therapeutic scene and extracting the data that best resonated with me (the audience) until I felt I had found the essence of the whole experience.

A developed data extraction sheet (see appendix 3 ) provided assistance in identifying and locating interview commentary regarding the setting, plot, characters and dramatic experiences which, when evaluated in relation to the other data forms (descriptive, observational and interview), evidenced whole therapy experience as more than the sum of its parts. Interpreting and extracting the interview data in this way, rather than transcribing verbatim and applying a standard content analysis and coding method, both avoided direct conflict with the 'performative criticism' method I have proposed (whereby the critic engages within the performance data) and what Shapiro \& Hunt (2003:923) call the "uniquely compelling emotional quality" of participant experiences, not present within silent text, within the resulting data and analysis. Together with the extra dimension of meaning in the 'human voice' (Smith \& Gallo, 2007), this provides an appreciation of not only what people said but also how they said it, and a vitality of words that is often absent within dry text. 
A natural complement to Theatricality, this enabled an analysis and synthesis that was both heuristic and dramaturgical in nature (showing similarities to both traditional dramaturgical analysis as described by Hare \& Blumberg (1988) and attribution theory within social psychology (Raven \& Rubin, 1983)) but goes beyond attribution of outcomes to either the characters (as in comedy and tragedy) or the plot (as in melodrama and farce) to confront the complexity of performances in a whole production where individual characters (including the researcher) tell their whole story, in both a holistic and authentic way.

Initially examined as whole data sets (each field site being analysed, synthesised and written up before moving on to the next field site) and drawing comparisons along the way, I found that as my own critical thinking developed, I could also modify this; effectively rewriting all of the fieldwork chapters to highlight those aspects (and characters) that I felt connected with me the most and would best enable me to communicate my impressions and findings with the reader. Now containing their effects on me at the time, and in response to my own personal growth and development since returning from the field, my final summary has become altogether more holistic.

\subsection{Dramatising the data using 'character monologues'}

To effectively communicate and dramatise the multi-level, multi-dimensional experiences here, I crafted character monologues from the 'live performances' of original interviews (and extracted meanings) and blended these with both descriptive and observational data, for participants to communicate directly with audiences, through and beyond the researcher (Jones, 2002). In developing this process, I listened at length to each interview; engaging with all the different sounds, smells and feelings of our conversations 'in the moment'. I found different possibilities for response, than working with transcripts alone may have done (i.e. transcribing and coding 'dead' text) and began to use the inflection in participant voices, their repetition and searching for the correct 'English' word, and the emotive choice of language, to help direct me to the most important replies and observations. An appropriate method of data (re)presentation, this allowed me to also weave the beliefs of the individual therapies and therapists, into the final performance. I actually tried several ways of doing this before I settled, often because of the pressures I felt from external discourse.

Conforming to the dominant research discourse of reductionist methods, that have been a constant and insidious threat throughout my journey, was never going to achieve my initial or stated aims for the research, but then I found it so hard to enact a better way while inhabiting reductionist environments and having to negotiate these, with their embedded reductionist 
philosophies while trying to maintain my own holistic philosophy (at home and University) to support my writing. I had been traumatised by the challenges of the system and, for a long time, this left me unable to (re)present anything with much clarity.

Was the task I had set myself a hopeless one, even if embarked with good intentions? Looking back on this, I am minded to question whether this is the reason that professors in my school advised that holistic research "couldn't be done" and eminent CAM researchers I met in Berlin also seemed to favour developing reductionist approaches (with pragmatic designs) rather than challenging these through holism? Certainly, it is very hard to "keep your head when all about you are losing theirs" (Kipling, 1895) and my prevailing culture, indeed the reason I felt compelled to embark on a PhD was because I felt CAM researchers has 'lost theirs' (or lost the plot, as I have written previously).

I did eventually have a collapse, but through my vulnerability I also found a 'space' and opportunity for new strength. I found I could lean (at last, I am usually the 'strong' one) on others around me, reach out more and speak about how I was feeling. Now connecting with myself, I was able to do this with my data too. I began to weave myself more into the study, responding to various statements that $\mathrm{I}$, the literature and participants had made, in a more honest and insightful way. I became a character in the story too, no longer hidden in the margins of my own creation or a remnant on the 'cutting room' floor. I had found my way back, and with fresh eyes and a learned spirit.

I returned to my whole participant narratives and made these central to the fieldwork performances; I have since built the chapters around these, and exposed numerous themes about the intent, role and impact on participant performance and experiences, from contextual factors in these events.

Challenging the ways research is currently designed and therapy events evaluated, I ha d again proven Read's (2005) assertion about space creating the potential for relationships, with both my own internal and external research performances. In this case, being in a more reductionist environment (e.g. UK clinical and research cultures) made it difficult to maintain and present a holistic perspective, mirroring also the participants' performances I witnessed (appearing more or less holistic, depending on the potential of the environments they inhabited). This has ultimately determined the type of engagement I have enacted, influencing both the quality of data gathered and the quality of analysis I later achieved. In dramatising my data then, I also dramatise my relationship with the environment (philosophical, academic 
and social) that created it. Knowing this alone wasn't enough and neither was seeking to actively re-engage with a more holistic paradigm. I had to acknowledge, before I could 'let go' of, the insidious grip that the reductionist paradigm had imparted on me. Only then could I begin 'healing' myself and succeeding in my work.

The resulting dramatisation of data is, therefore, a contextually driven and culturally located one that speaks both of the phenomena under study while also creating and sharing its full meaning for these characters, thereby unmasking "the spectacle and whatever goes into its making" (Patsalidis, 2011). This alternative way of "interpreting and presenting the results of an ethnographer's work" (Denzin, 2003:13) represents my earnest attempt towards holistic, heuristic, rigorous and reflexive research.

\subsection{Photographic images of set designs}

Providing an additional 'way into' the data I have also chosen to include some of the set design sketches (reproduced) and photographic images (triptychs) within each of the relevant fieldwork chapters. Referring to these has helped me to remain connected to the various settings and, as already mentioned, I found the images included in Kleinman's (1980) text to do the same. Therefore, I offer them to the reader as an additional way to engage with some of the unique complexities and the whole presentation of each setting and navigate the space in a more sensory way than text alone may achieve.

Their use will likely affect some readers more than others. I know, for example, that my primary supervisor found them of little benefit, and even to 'get in the way'. This is not my experience, but I cannot know which of us is right (we possibly both are); which again fits with the interpretivist and phenomenological nature of this work. When I first developed the idea for this study, taking pictures of the scenes was not actually something I had considered, but was 'persuaded' into after listening to advice from a senior academic at the IN-CAM conference I attended in Vancouver, in 2010 (where I presented Theatricality for the first time). She made the case that such an act could only add to the engagement of the reader, so I leave it to the individual to decide whether to connect (or not). This is not the only/primary source of data presented, so I am not unduly concerned, beyond allowing those with a desire to see and connect this way, the opportunity to do so. 


\section{Chapter 5 - Staging the research production (Methods II)}

Producing this research was a complex event with integral impact to my whole enactment and the resulting data I collected. I had never set up and run a study of this nature before (across multiple sites and countries) although I had produced large scale local amateur musical theatre productions and even directed a national CAM therapy conference, which I used as a basis for my performance here. By this time it seemed quite natural to perceive and frame my construction and composition of the study also, within the perspective of Theatricality, and this proved an easy way to conceive the linking of different aspects within the overall event demonstrating the universal potential of my methodology.

\subsection{Selecting and recruiting performance venues (participant clinics)}

The selection and timing of recruitment and participation for Centres in the study was both a carefully orchestrated event and a crash course in serendipity. My remit was to compare complementary health centres in UK and Europe, and I had to ensure I could engage an appropriate sample within my own physical and funding limits. I began pragmatically, by first identifying possible countries to include, and then sought contacts and clinics from there. From a formal perspective, this meant incorporating elements of both purposive and opportunistic sampling; methods more often associated with social science than healthcare research. This fits well with the underlying holistic philosophy of my study (that everything is interconnected and happens for a reason) as it creates an almost karmic (meant to be) outcome; I trusted the Universe to offer me whatever I needed (a theme that is embedded in all aspects of my life).

Given that I have no fluency in any other language than my own (English) and I had decided to collect data by means of both observation and interviews, it was essential to select fieldwork locations where English is spoken widely. I also wanted my sample to reflect as much diversity in context as possible. I felt that a good geographical spread would prove advantageous in this, all the time aware that for the sake of governance and visa issues (see later) it would also be desirable for these countries to be within the European Union (EU). A 2006 EU Commission survey showed that English speaking levels in excess of $70 \%$ of the population were to be found in the UK, Ireland, Netherlands, Denmark, Sweden, Malta and Cyprus. As fortune would have it, I had access to personal connections in the UK, Netherlands, Denmark and Cyprus who had offered their assistance with setting up the study, when the time came. These were all contacts I had collected through my personal, professional and research activities, so 
continuing with a pragmatic, and karmic justification, I a pproached these personal connections first, and recruited them as intermediaries to assist in the set-up and execution of the study, in those countries.

In terms of the clinics my intermediaries found/suggested for me, these represented both purposive and opportunistic sampling, as many were either known to the intermediaries or (with the exception of Denmark, where they were not personally known) they were part of an existing electronic/email network). This made their recruitment easier - on both sides - and I also felt I could trust Centres recommended by these sources to be safe for me to engage with. Equally as they felt as I was coming through a reliable source, I was also trustworthy to be allowed in. I had no prior knowledge or preconceptions about the nature of any of the Centres that were suggested or found for me before they were approached (only the range of therapies they provided) other than they were all private clinics and all delivered a combination of therapies. After these initial introductions I conducted all recruitment personally, through lead practitioners or managers, and on the telephone, via email or Skype meetings.

The UK site came about first, through two connections. These were a historical connection I had with a therapist who no longer worked there, but through him I had a potential introduction to the current senior practitioner. Added to this, my own therapist (she delivers my massage treatments), who was aware of the nature of my proposed study, had spoken on my behalf to a friend of hers who currently worked there, and she had said they would be amenable to an approach from me. The clinic itself was situated about 15 miles from where I lived, and this seemed like a good opportunity, so I contacted them by telephone to enquire whether they would be willing to meet with me to talk about participating, and then followed this up with a series of visits to discuss my ideas for the study and the nature of their participation, and also to receive guidance from them on developing an appropriate time scale and level of intrusion into what was a 'working therapy practice'.

The second site I recruited was in Cyprus. Serendipity was a clear player here, as my contact with this clinic came about through a quite 'random' chain of events. Shortly before I began my PhD studies, my mother (who had worked in the office at my GP surgery since I was a small child) decided to finally retire from her work. One of the last tasks, before she retired, was to settle and orientate a new GP into the practice - from Cyprus. He had immediately taken to my mother and was so grateful for her attention, he had offered to help her in any way he could, in the future. So she called in the favour and asked him to enquire from his father (who 
was still a GP in Cyprus) of any reputable clinics or practitioner that may be suitable for approaching into my study. As it turned out, his mother (in Cyprus) had been having acupuncture treatment at a clinic near to where they lived, and so his father spoke to the practitioner there, who contacted me directly, eagerly offering his centre as a participant. Unfortunately, when I began to schedule participation, it transpired this practitioner was going to be on study leave to Australia for several months and just couldn't fit it in. So instead, he offered to help me recruit another site and proposed (and contacted) a colleague of his, in another part of the island, on my behalf. This practitioner was interested too, but more cautious, and only after several discussions and deliberations, did he agree it would be appropriate to participate. I was just glad to have secured a site here - and in July!

My route to two therapy centres in Denmark was a colleague whom I first met during a breakout session at the conference I attended in Berlin. He had (foolishly) offered to assist me in setting up the study there, if and when I was ready. So when I was ready, I emailed him to ask for his assistance. Already part of an established network of CAM practitioners and researchers there, he put out a call to his mailing list and received seven enquiries back from practitioners keen to know more about the study and discuss this with me. All of the clinics were single practitioner models, meaning they were staffed by a single therapist who was trained to offer patients a range of different therapies. I was concerned to allocate both my time and funds in a potentially risky situation (what if I went there and the practitioner was sick and I lost my access to therapy and to patients?) but felt that if I could select two clinics to work with, I would have some security against a null response. Of the seven clinics, who showed interest, two were quite close to each other (in neighbouring districts) while the others were more widely spread (geographically). Again, considering the pragmatics of the situation (I would be completing all of the data collection on my own), I felt I should enter discussions with the 'closest two' first. These were very keen to be involved, for different reasons, and seemed to provide good examples of the dominant model in this location, so I felt no need to look any further and agreed to their participation.

Holland was the last place where I located and secured a centre to work with, despite being the first suggested to me, by my supervisor. He had done a lot of work with a colleague there, and felt the centre where she practised may be a good one for me to approach. Not wanting to pass me her details directly, he said he would contact her for me, to 'set the ball rolling'. When he eventually got around to this, she responded that I should approach a Professor colleague of hers first, at the University of Leiden. I did this, explaining my proposed study to 
him and asking his assistance in identifying suitable clinics to approach. He actually suggested three to me, including the one where my supervisor's colleague worked. I decided to contact this one first, out of courtesy really, and was greeted so enthusiastically by the lead GP there that I never considered rejecting this in favour of another 'unknown'. Whether this was chance or serendipity again, all of the pieces had fallen into place.

A much easier task than I had anticipated, this further suggested a 'karmic synchronicity' to my work, as such a smooth and successful recruitment should have been illogical! I had quite quickly identified and recruited suitable clinics (given my initial remit of providing a combination of therapies) in 4 different locations, each providing me with a unique opportunity to engage in different presentations of therapy. It felt like part of some great master plan (and not one of my writing).

After this, contacts between myself and the clinics discussed in specific details their individual participation. I supplied them with appropriate documentation for further consideration, prior to formally accepting them into the study and we discussed other aspects, to aid planning, such as how many practitioners, what modalities are offered, how many patients are seen in an average week etc. As individual centres, I found these of interest in themselves and as gateways to practitioners and patients. The clinics were all keen to agree not only the duration, but also the proposed timing of my visits so they could ascertain whether it was possible, from a practical perspective, to accommodate my presence on site. Lead practitioners were also concerned to know what levels of intrusion would be required, the numbers of patients I hoped to recruit and, in some cases, the kinds of information I would be seeking from these. All of the clinics appeared intrigued and interested to see what my study could bring to their attention, feeling that they had the potential to directly benefit from 'seeing the clinic through their patients eyes'. I supplied the clinics with the relevant information sheets and assured them this study would not be making any value judgements about the quality of their centres or the treatments provided in them and they all agreed.

I never intended it to be this way, recruiting more out of chance, opportunity or serendipity, however the sample that I ended taking forward into the study proved an illuminating mix of diversity; both of place and provision, in model and modalities. I was always looking for the whole complex and unique in therapy anyway, so it really felt no problem to me that there was no potential for generalisability of findings. In fact, I was pleased this would give me a real opportunity to explore the heterogeneity in CAM that others before me have identified as a 
problem in conducting research, and attempt to understand and 'to solve their riddle'. I saw celebrating these differences as being what life on the margins was all about anyway?

\subsection{A touring production}

As this study always intended to value and utilise comparative study of data from complementary health centres in the UK and Europe, it was always going to be necessary for me to recruit and spend time working within a number of different centres in different locations. Due to the inevitable complexity involved in running a study across 4 countries, both within and outside of the UK, I explored practical, ethical and legal issues at an early stage, factoring any related activities into my lead-in time.

I was welcomed on site by all the clinics for a period of up to 4 weeks at the outset of the study. I had worked out a protocol for the various elements of data capture within the study, and a planned sequence for completing these within the allotted timescales, beginning with TFM mapping/capture of the various internal and external spaces, followed by formal observations (including some treatments) and interviews with patients and practitioners.

Prior to undertaking any periods of fieldwork, I conducted the required risk assessment procedures, with particular awareness of local policing and healthcare provision, should I find myself in difficulty in an unfamiliar location. Background information was available on the internet, and I received advice and support from the UK based Foreign and Commonwealth Office (FCO). However, I found these were no substitute to having dialogue with people 'on the ground', approaching and gaining support in this from both my intermediaries, clinical leads and selected senior academics within potential host countries, with the latter's advice also including matters related to legal and ethical policies and procedures.

Within the UK centre, this plan worked well, and I intended to replicate this at the other study sites. Of course a true replication could never be possible, due to the innate variability in therapy provision. However, I concluded all the UK data capture easily, even having many opportunities along the way to mix with the practitioners 'off the record' and get to know them better. Within the final week on site, I only had a few interviews arranged, so was able to explore the immediate location, the people and its amenities. Having successfully completed all of my fieldwork, and interviewed 8 practitioners and 10 patients in the time scale, I felt confident that this plan would also work well during the remaining sites, although feedback from the clinic suggested that 4 weeks on site, full time, was 'more than enough'! 
The Holland centre had more rooms to describe (across 2 sites) and more practitioners to interview than I had encountered in the UK, and so instead of being 'more than enough', I fully expected to need the full 4 week time scale here. It wasn't long before I found that this clinic actually operated across 3 sites, which depending on the mode of transport were either 30 minutes apart (walking) or 15 minutes apart (bicycle). Juggling my attendance on each site, therefore, had the appearance of being present in each distinct setting part time, which I feel had a positive impact on the practitioners here, as they never reached saturation with the level of intrusion as I found had been the case in the UK. While this did put some strain on my timetable, I performed flexibility around the availability of treatment spaces, practitioners and patients, often attending 2 of the 3 sites in any given day. This general approach was, therefore, not as relaxed as the one I enacted in the UK site (that was in a single site) and here I had much less opportunity for spending time in the social company of the practitioners, alongside observing them formally. This is not to say that I spent no time with the practitioners here as there were structured staff coffee breaks, where all practitioners would meet informally, and I was also invited on a couple of social events away from work. I did, however, feel a further week on site here would have been beneficial and could have enabled data collection to be more relaxed, like my experience of the UK site. Despite this, I completed all the data collection in the allotted time, interviewing 17 practitioners and 19 patients, and got to know the City well, as an active participant of the bicycle commute culture.

After running the study in 2 different centres, with different levels of complexity, I applied a new level of knowledge and experience to the remaining sites in the study. I used some of this knowledge and experience, gained from fieldwork in UK and Holland to manage a change at the Cyprus centre as, since recruitment, I had become aware that the centre would be closing for the final week of my stay, to facilitate a holiday period for all the staff. Knowing the size of the site and the number of practitioners, I felt confident that I would be able to complete all of the required data capture within the 3 weeks that the clinic would be open, and was therefore present on site for 3 weeks, using the final week to explore the wider locality in which the clinic is situated.

From this experiential knowledge, I also amended my plan to stay in Denmark to just over 3 weeks, splitting this time period between the 2 separate small clinics I had recruited there, which positively impacted on my projected fieldwork costs, slightly reducing these.

The conditions of my schedule within this study required co-ordination of planning, risk management and finance, alongside managing complexity within the study design, and a 
number of issues from outside it. The intricacies of how I ended up where I did, when I did, may not seem as important from an audience perspective as they were to me, however they were critical to the overall performance and success of the study, underpinning my ability to be in the agreed places at the agreed times, completing the agreed nature and level of work.

\subsection{The production team}

As principal investigator, I have been the central character in this study, supported by my two supervisors and many other individuals. In a kind of 'method acting' I became embedded in and embodied this study, bringing training and skills from my previous life experiences as a practising CAM therapist and a trained amateur theatre practitioner together with new ones, gained through academic exposure, attendance at a number of workshops and conferences and other sources, to create something I felt would be capable of engaging with the whole complexity and unique natures of different therapeutic worlds. I also acquired awareness and knowledge of issues pertinent to my research, particularly in regard to ethical engagement of the public in research and conducting research overseas, which I did not have before, but further enabled the creation and staging of this unique production.

This unique synergy of components, and my role in enacting these, has been key in this whole study becoming the study it has and I also believe that any other actor/researcher stepping into my role would likely do things different! For example, being a practitioner, I empathised with practitioners during the development and execution in a way I do not believe I could have otherwise, ensuring sensitivity in my observations and interactions with their patients. Indeed, during feedback after participation, I learned this had proven a real asset to both the initial recruitment of clinics and their willingness to participate in research studies, in the future. I think it is true to also say that in giving myself into this study, it has taken much from me.

With equally critical roles, were my supervisors whose early enthusiasm first encouraged and enabled me to conceive what turned out to be a challenging, exhilarating and heuristic venture, despite not being CAM practitioners nor (as far as I am aware) theatre practitioners. I credit these with creating enough 'space' for me to discover both who I was and what was important for me to achieve in this process (which wasn't always what I thought) by their stimulating and learning targets. Sometimes they found me impenetrable, but that never stopped them from trying to reach me; always aware of my potential (even when I began to doubt myself). I lost count of the number of supervision sessions where my second supervisor made me cry - often by her support rather than critique. Indeed, I doubt there was ever a bigger critic of my work than me! 
Other supporting members (equally critical, if differently so) were my intermediaries within each country, whose nature of roles and levels of commitment naturally varied across the sample. The common primary function of these was to identify suitable (and safe) clinics for me to approach regarding participation, although other duties occasionally came into play such as assisting me in securing suitable accommodation while on site or other social participation, again dictated by the variable nature of these individual characters and settings.

Each field location also had its share of extras, those offstage characters that were present at each field location, yet not directly cast within the scenes. The roles and value of these, while not being specific to the study, still came to be integral to my overall performance, and thereby impacted the research, either framing the research structure, or else influencing my daily patterns and psychological wellbeing - particularly during periods away from home. Mirroring theatrical production (which I also found to be the case within the various therapeutic practices), every member of this cast and crew held critical roles in the whole production and performance of this research, and I consider myself very fortunate that those fulfilling these roles were so eager and able to assist and support me.

\subsection{Seeking ethical and governance permissions}

The actual process for ethical approval began prior to any clinic recruitment. As a UK citizen and therefore member of an EU state, I did not require any additional visas or permissions to work within other EU countries, and pragmatically used this position within both the design and execution of the study. Many of the laws pertaining to research practice and data protection in the EU are also common to members in the UK, which was an asset in that my approach to ethical approval and protection of data could be guided by current UK regulations.

Checking the validity of these permissions with the relevant intermediaries (once I knew which countries I would be working within), I found that approval for the study granted by the School of Healthcare Research Ethics Committee (SHREC) at the University of Leeds, would be sufficient for my work within both the UK clinic, and also the overseas centres. Upon the advice of a senior academic in Denmark, I also made an application to Datatilysnet (the Danish data protection agency) as this is regarded as a general requirement for research conducted in Denmark and is in addition to any granted by a University. Assisted by my Danish intermediary (the Datatilysnet website has English translations on it, however the application form which can only be submitted online, is in Danish) who translated the form for me 'live' as I was completing it, I made the application. However, the response I received was that as I was both 
the principal investigator and the data handler, and that the data would be processed in the UK, I did not require their a dditional consent, and the legal standards of the UK would apply.

\subsection{Financial backing for the production}

Also part of the 'staging' process, I had to secure funding for the final production. As a crossEuropean study of significant field duration, the major expenses to be accounted for here were flights to, and accommodations within the identified locations and countries of my choice. So prior to actually committing to the study, I needed to meet the cost of executing it. Gathering the required information for an external funding bid took an inordinate amount of time, but somehow encouraged me that my concept for study was achievable, as I began to fantasise about the places I could go, the people I could meet, and the unique insights I may gain from this. I also began to realise how this study design could enrich me as an early career researcher, and contribute to my developing an international network for future collaborations.

Potential external funders for this study were limited, however, as the majority of funding bodies all had restrictions that prevented my eligibility for funding (such as not already being a post-doctoral researcher, not wishing to cover additional staff costs etc). Eventually, I identified the LCSP-ERD Charitable Trust, a small research charity whom I had previously done some voluntary work for, as being a possible route to funding and indeed these were able to provide me with the funding I required and allow me to commit both to the concept and the research plan. Without their backing, this production would likely have never hit the footlights, or else it would/could have become something altogether 'less' than it now is.

\subsection{Scheduling the fieldwork performances}

Taking my own perspective as a practitioner and having also spoken to a number of colleagues, it seemed that up to 4 weeks would be a reasonable amount of time to permit intrusion into practice, so in the absence of any other guidelines this became my benchmark. I was of course willing to negotiate this with participating clinics if required (this was discussed during recruitment with clinics) or modify the timescales based upon learning from the field (which I did action through the various phases). This proposed period also seemed reasonable in terms of cost implications as a much longer duration is often required to gather this level/quality of field data, and this time scale seemed a workable one as I had determined that I could expect to gather my descriptive, observational and interview data within the proposed time frame of 4 weeks (from a pilot within my own clinical space). 
Througho ut the study, I carried out 4 periods of fieldwork. Pragmatism was a driving force, as I scheduled the 3 overseas visits (each of up to 1 month) first, fitting these around local festivals, weather predictions and the availability of the clinics. The Holland fieldwork was conducted around the Easter holiday period (mid-April), Cyprus during the early part of the summer (mid July) and Denmark during the latter part of the summer (mid August). The UK clinic had no interest in being scheduled around these times, as this would leave their staff free to take their usual holiday leave, so I offered the UK site either March (before I headed off to Holland) or October (after I returned from Denmark) for their participation. As they preferred not to wait until the end of the year, they became scheduled first in March. In truth, these periods of fieldwork were structured a little too closely together such that I barely had time to catch my breath (particularly between UK and Holland, and later Cyprus and Denmark). However, the intensity with which I was able to engage with the whole 'touring production' did give me some unique insights into both the phenomena of therapy - and the phenomenon of research!

\subsection{Recruiting practitioners and patients}

Actual participant recruitment (for patients and practitioners) at each site began with the display of posters and study information sheets (in most cases before I arrived on site and began working in the clinics). Although no documentation was completed until after I arrived, I understood the majority of practitioners to be willing participants from discussions with the clinical lead at each centre, often being awa re before my arrival what their participation would require; something that was also discussed directly with them on site. I took this as a good indication of potential patient uptake too.

For practitioners, the formal recruitment was openly purposive, as these were all resident staff members at the various sites. Fairly easy to co-ordinate in the UK, Cyprus and Denmark, due to the organisational structure of the clinics, this presented more of a challenge in Holland simply because these characters were spread across 3 separate locations. Each was done personally by me on site, adhering to the ethical permissions agreed by the University, whereby consent was freely given and participants had opportunity to ask questions before committing. Of a potential 33 practitioners across the entire study, I was pleased to find that 31 agreed to actively participate, consenting either to my documenting their therapy spaces, observing them delivering treatment and/or participating in an interview.

Patient recruitment was intended to be opportunistic, although in some places became purposive (where practitioners felt a need to control or restrict access to certain patients), and also rather less predictable. This was easier in some locations than others, and I found 
patients within the UK generally the most reluctant to participate, often not having (or willing to give) the additional time it would take out of their day. Conversely, those in Holland were most eager; a number of these responded to the posters and information sheets that clinics put out prior to my arrival, leaving names and contact details. In Cyprus, all patients recruited were selected and invited to participate by the lead practitioner, based on his assessment of who may be likely to be visiting the centre during my time on site, had sufficient English language skills and was stable enough (health-wise, in his opinion) to engage with me. I made sure, however, that each potential participant had opportunity to read the appropriate information and ask any questions before offering or rejecting their consent. Finally, in Denmark I received plenty of interest, via the therapists prior to arrival, but had to reject a number of those, as many who wanted to participate did not want to read the study information first (which was a part of my ethical permissions), which reduced the number of active participants to the lowest across the whole study. I found valuable lessons in this (for future work).

Rejecting a 'systematic approach' to patient selection felt both a natural and pragmatic choice, as I could not rationalise silencing any single voices, within my study model. Ultimately, the integrity of the study was maintained and the protection of participants was never compromised, so I came to accept this as part of the 'realities' of my research practice.

An interesting note is that the enthusiasm and commitment of the lead practitioners to the study seemed to be mirrored in the enthusiasm and commitment of patients to participate. Strategies such as the patient information sheet 'tear-off slips' were most successfully used in Holland and not very successfully at the UK site (who requested them), which an appraisal of their placement within the whole reception-area staging gave some clear clues to. At the UK site, these were initially placed on a low shelf out of the natural eye line of patients, whereas in Holland they had been clearly displayed on top of the reception counter. Waiting room posters (at least 2 in each waiting area) were also more clearly displayed in Holland and patients seemed generally much more aware of the value of study. In Cyprus, no obvious advertising was displayed (for fear such literature may disturb the carefully 'balanced energies' within the centre), meaning patients could not just volunteer themselves, although the lowest number of participants was actually from Denmark, due to the issue of patients not wanting to be bothered reading any required information and consent form. This small aspect of the study process came ultimately to have a large impact, both on the data that was achieved, and not achieved. There is also much rich data contained with these 'process' observations. 


\subsection{The role of researcher}

I found that performing the role of researcher in this study could be seen as bearing many similarities to performing a role within theatre, where every aspect has been and remains a vital part of the whole event. Also experiencing a personal and professional growth, throughout this process, can be a consequence of exploring oneself through engaging other characters. The effects for me became apparent the further I entered into it and are reflected in the quality of data I was able to collect and how I have come to connect and communicate these. With different research experiences and results in each location, the only constant has been myself, and yet I was also moving and developing (so not really a constant anyway).

The position I started from was to accept CAM as an episode in real life and that, just like real life, these are complex events. I wanted to find a way to illuminate this complexity so research could begin to better reflect participant realities, when evaluating processes and outcomes. In seeking to illuminate, value and capture the whole performance of therapy and reveal those aspects normally overlooked I have captured and illuminated my own performances also, as a researcher, and through this I have explored my role in the final production. This final thesis has been a challenge to produce - because of the complexity of the task and the complexity of my life - neither of which I can do much about, except face it.

After my initial submission, almost this entire text has been re-written; once I learned to pick myself, and much of the value within, up off the cutting room floor. My first attempt, according to my supervisors and examiners, made it impossible for the reader to connect either with me or with the data. But since then I have reflected, suffered, and reflected some more and I hope this written performance will finally communicate this journey, stimulating other researchers (and research) to seek, appreciate, value and challenge their own ideas around complexity and holism in both CAM therapy and research.

\subsection{Madness in the method}

In the generation, interpretation, reflection, analysis and synthesis of this data set, an appraisal of the whole performance of therapy is created with therapeutic experience not based on externally measureable outcomes, but on internally subjective ones, revealing a different side to the phenomenon usually overlooked. With "an intrinsic transferability" (Avis and Freshwater, 2006:223) these outcomes may resonate with practitioners and patients of other therapy approaches, raising the potential to identify commonalities and contrasts 
between them, stimulating further study. A unique strength, this gives my study potential application on a universal level.

Starting out as a method to focus on bringing the contextual variables of the setting to the foreground allowed the interventions to take a background position instead, creating a 'space' for the human dimension to occupy the 'centre stage'. A complex process to enact, perhaps as much as the performances of therapy I witnessed, my engagement with each setting from the planning, through execution and into performative writing has been subject to the influence and impact each contextual setting has made on me. Indeed, my own performances could be appraised and critiqued in much the same way, also influenced by the variables in the setting (although this may require another PhD). As with those I observed, I found that while some of these variables naturally resided in the background, I was still able to glean much about how I used my spaces and actual experience with and within these as much as the participants did, supplementing each subjective story.

After the fieldwork, a further challenge was managing the data it produced, although writing a coherent report that would suitably capture and reflect my experiences of the 'whole story' proved undoubtedly the most difficult part. Utilising a holistic perspective of this, I can identify this final period was also a difficult chapter in my life, with increasing distance between myself and my supervisors, and family illness and death (that I took primary charge over) immediately prior to my first submission, doing little to enhance my confidence. Then during the beginning of my correction period, I was thrown into year of extreme instability in the health of my youngest child where repeat emergency hospital admissions (and hospital stays for me) became the norm. Still, I was intent on being a 'good student' and not disappointing all those who had invested in me to this point (including the participants), that I 'bullied' myself to continue long after I should have stopped - until I had a n effective breakdown.

Viewed from within holistic philosophy, these events are as connected to the final product as my personal and practice history: the 'show' I witnessed in Berlin, the research bias that prompted this 'reactionary' work, and the data this generated. The truth may be that challenging the system 'alone' created too many personal problems for me to cope with - a side-effect, if you will, that is enough to deter any similar attempts (alone) in the future! Conversely, being stretched to a point where I became truly vulnerable has helped me to connect with myself, and my feelings, in a way I have shunned for many years (probably part of why I became a therapist is the focus on others, rather than myself). Where possible, I incorporate and honour the responses these events invoked on my work, as a seamless plot 
within my writing (making this also a part of my method). Thus my thesis is as much about $m y$ journey to develop and apply holistic practice within my research as it is about the real-world therapies, people and places I studied.

Refining these ideas into a workable method, therefore took many attempts (and almost my sanity!). When I first submitted this thesis for examination, I was keen to include every voice, every participant with something to share. I was concerned not to artificially 'reduce' the data to make the point. But in this inclusivity I lost the point, myself, my supervisors and (I suspect) the examiners as we all became buried under a mountain of bland, generalised, sanitised repetition that left little room (or 'space') for true, insightful analysis. This was disappointing, on many levels, but most of all because I had actually found these performances of CAM to be fresh, unique and richly complex episodes - the opposite of bland, generalised and sanitised.

This brought the concept of research, restricting engagement with complex events like these, very close to my door. This had been something I was going to actively 'resist and reform' in my work, but somehow I fell into enacting instead (at least for a while). Simply 'not wanting to' be trapped inside a more descriptive, than interpretive, presentation of data clearly wasn't enough to avoid it. Somewhere within, the 'strait-jacket' of conventional, scientific, positivist, academic thinking, inside my 'home' University environment and the influence of those I met at national and international CAM conferences/events, served to distort me as it has others.

Like someone who is sedated but not dead, this made me more and more paralysed; watching my ideas and inner space succumb to the background discourse around me and unable to do anything about it (despite having engaged therapy centres and locations and immersing myself in their cultures) until both I, and my potential, were completely destroyed. I nearly gave up, feeling that I had underestimated, maybe even been naive in challenging the status quo.

Fighting my way back has been a lesson in itself. I first had to admit my failings (inside and outside of research) to see how to reconnect with myself, and then the conviction that brought me into study in the first place. I became mindful of the power exerted by my external, intimidating discourse, and looked for the power to resist this. In a truly healing process, this came from within, not without. I revisited my fieldwork data and instead of merely representing this I reconnected with it, which opened up a whole new 'space' for reflection and analysis that became the fieldwork chapters I now present (Chapters 6-9).

Much like Paterson and Britten (2008), I looked again at the performances of my participants and from this I crystallised the essential things in each performance, and discovered aspects of 
this care that other studies had failed to reveal, or reveal fully. Far less prescriptive than my approach to data collection had been, I found this a liberating task and, through this, generated a 'better' route to understanding and presenting my findings. Emerging, I have created from complexity a coherent performance, just as a producer or director in the theatre also creates a coherent performance from their production parts. All fragments of the 'whole data set': the descriptive notes (TFM) and photographs of therapeutic and local environments; clinical observations of treatments and interactions (stage directions); and audio-taped semistructured interviews with practitioners and patients (dialogue) are also part of my 'whole research experience'. Perceivable also through the frame of theatricality, this 'sets the scene' for my study and the whole context, to interact with the human dimension congruent with the underlying holistic paradigms of CAM and theatre.

I had entered the process of research like an investigative reporter; to go undercover in the arena of CAM research and expose the hidden realities of practice, so 'research reform' could take place. These expectations, while laudable, were something that may have been as unrealistic as they were idealistic. I began to perceive many of those 'stars' who currently dominate the literature (and research budgets), simply by adhering to dominant methods, as actively blocking any potential reform which made me question my whole potential. I genuinely wanted to reform CAM research with and alongside the $m$, not in opposition to them and so followed their lead, into 'opposition with myself'.

It has been a personally overwhelming and challenging effort to provide an accessible way to think holistically about research, and the meaningfulness of this to the future practise of CAM research and therapy; but I hope a worthy one. As not even my intended destination when I began this journey, writing this has required additional courage I was not aware I would need to possess, however, I am a curious individual, and knew that something was missing, overlooked, within this body of work, and that if I could apply a 'holistic lens' to this, I may just find an alternative answer. In following this path, I demonstrate an earnest attempt to apply the concepts of holistic practice to the practise of research, integrating theory and results as I honour both the 'seen and unseen' aspects of this larger environment. As an act of knowledge production, this illustrates just how much, and how little, we currently know about the delivery and experience of CAM.

Within this, the greatest influence both to the therapies I observed, and to my research, have been the practitioners who deliver these. Always and absolutely acting in the best interests of patients, these characters were 'gatekeepers' (in the sense they enabled my access to the 
settings and those who perform there, and also restricted this where they felt any 'intrusion' may be inappropriate either for themselves, their patients or most importantly therapeutic outcomes) which my own status as a CAM practitioner helped me to empathise with, accepting any influence as 'real-world' research. I did not seek to control this, but rather embraced it, until I became charmed by these people and their way of life. They were my starting inspiration and it is my commitment to bring a fair and honest representation of their work, as I critique the whole concept of CAM research; which has sustained me through to the end. This engagement even supplied a new and different energy to my work, and an unexpected sense of belonging, allowing me to grow as a researcher and a person. 


\section{Chapter 6 Act 1: "The In-Betweeners"}

My first therapy setting was a private multi-disciplinary therapy clinic in a large Northern UK town. Behind the external façade this looks more like a regular physiotherapy clinic or private dental surgery that Complementary Therapy Centre, and offered a fairly mainstream model of care inside. Here, a team of 9 practitioners (each trained in a different modality) offer a range of therapies including aromatherapy, reflexology, TCM acupuncture and herbs, Pilates, massage and manipulations, Alexander Technique and hypnotherapy.

To aid a sense of the physical space, set plans are as follows:

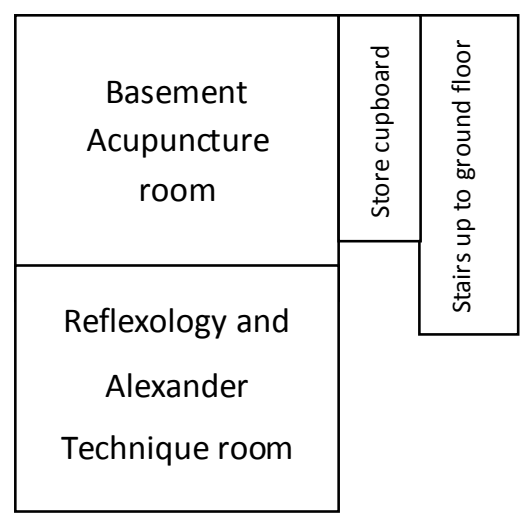

Set plan 6.1: Basement

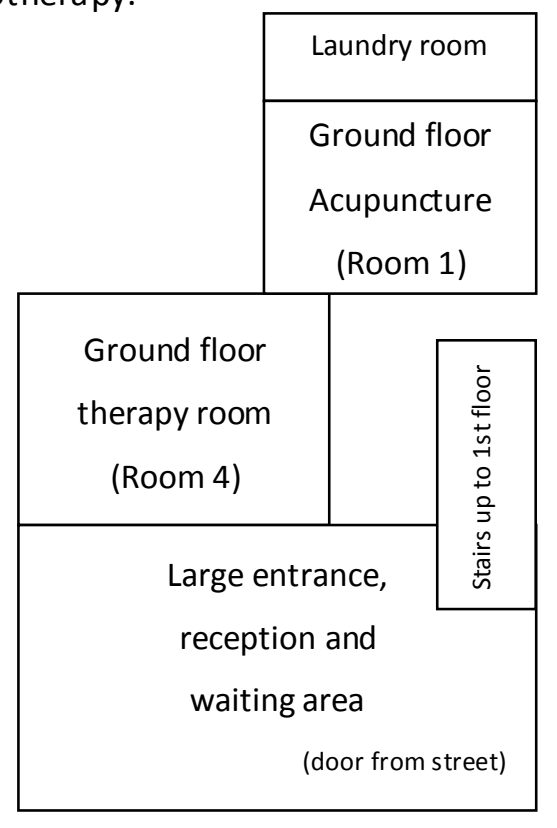

Set plan 6.2: Ground Floor

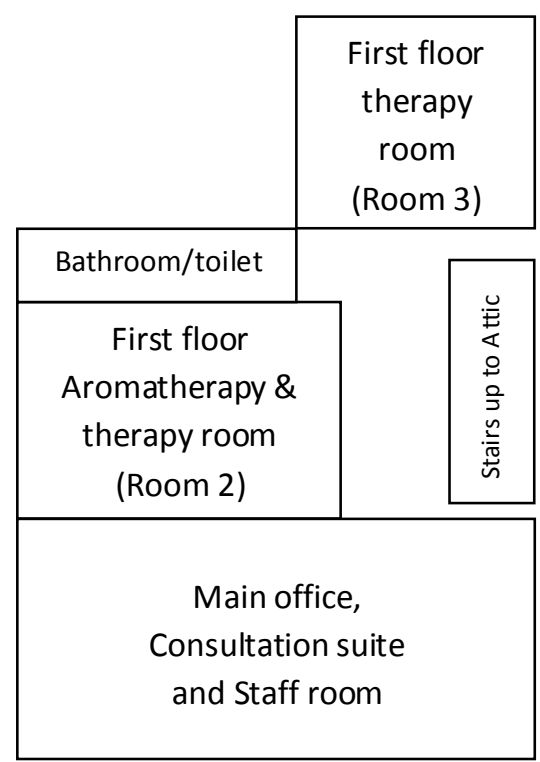

Set plan 6.3: First Floor

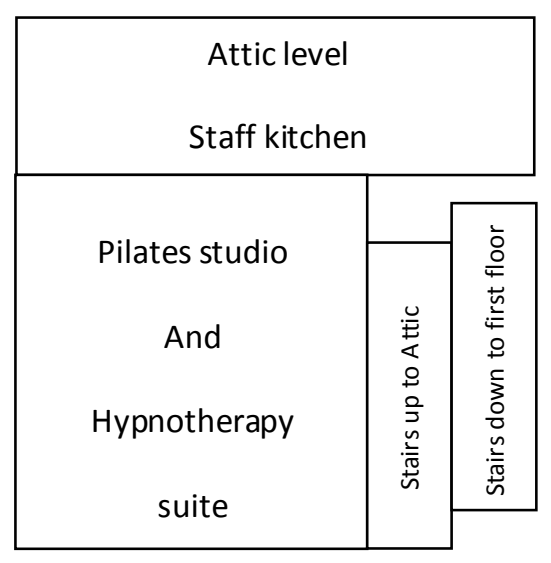

Set plan 6.4: Attic Level 
I was on site here for four weeks, during which time I made observations, both general and of private therapy events, and interviewed eight practitioners and ten patients. I did not know what I would gain from these prior to committing to them, other than the potential to create a picture of therapy performance and impact, that was unique to this context.

I do not present all of this data here (these interviews alone created 23 pages and 11,000 words of dialogue alongside a whole notebook full of observations), but instead selected episodes or scenes that I feel best expose, express and explore the holistic nature of this whole performance and my evolving subjective/objective engagement with this (including the tensions I experienced between being objective and subjective, in my engagement). Observational accounts and carefully scripted character monologues sit side by side here, aiming to bring a living dimension to the data and each selection highlighting either a common view or something unique about the relationships between these characters, plot and set. My critique within this is as a 'participant within' rather than 'foreign afar' (performative criticism - see Chapter 4), putting myself into the data and thus creating potential for a richer source of learning, from this event.

\subsection{Act 1, Scene 1: A 'model' clinic}

I met Helen right at the start of my time here, and she was one of the first patients to agree to participate. A smart and 'mature' lady, she was introduced to me having been a regular here for many years (and therefore seen many changes over time, both in the space and the personnel). We had a brief conversation about the nature of my study, and what participating would entail, and Helen agreed to take part in both a therapy observation and interview the next week.

On the day of her participation, Helen was already waiting in the simple reception area, when I arrived. She was seated on one of the black framed metal chairs, her back to the wall opposite the main door, chatting freely to the receptionist - their easy rapport seeming to somehow brighten up this otherwise still and stark space. I greeted her and said I would be observing her treatment first, and we would do the interview afterwards. Then I exited up the old staircase to the first floor main office/staff room, to prepare myself and join with the practitioner I was to accompany, until her appointment time.

I entered the treatment room, together with the practitioner, to find Helen seated on a brown plastic chair at the end of the room already wearing just her bra, knickers and tights. She had no modesty gown or towel, and looked a little uncomfortable (but accepting) under the bright 
fluorescent tube lighting. I glanced around the room and took in the space. Every item was clear and apparent; from the simple and utilitarian furnishings and the 'dated but working' electrical therapy machines that lined the walls to the miniature plastic clock on the windowsill and the lack of any warmth (even the towels were cool in navy and white)! A brief "Hello" and Helen immediately stood up facing the wall (she clearly knew the drill). The practitioner approached her and straight away began palpating/squeezing her upper shoulders as they chatted about how she had been and what she had been up to since the last treatment. He briefly stood back to determine any imbalance, before inviting Helen to sit on the therapy bed, where he continued behind her with some firmer massage.

Throughout, their chatter was mostly dominated by topics of DIY and painting, and the implications of these for Helen's condition. I sensed a history to their relationship, but failed to spot any real empathy or warmth within this (like the general staging and feel of this place). I sat quietly on the chair, where Helen had originally been, as together they completed what looked like an almost mechanical process of osteopathic style manipulations, repositioning (including lying on front, back and sides), and more manipulations (modesty now covered with a towel, just) before finally the practitioner took the industrial sized massage gel/cream and applied a general massage to the manipulated area. Within a few minutes, he had dried his hands on a piece of tissue paper which he disposed in the nearby waste bin and was placing electrotherapy pads on Helen's lumber and upper thoracic areas. He recovered Helen with the towel before leaving the room, advising that he would see her again next time. Helen lay still and silent for around 5 minutes, the machine buzzing and vibrating on her body. Then, one of the massage assistants came in and removed the electrotherapy pads. She proceeded to clean and put away the equipment (ready for the next patient use) in full view of Helen, who was now getting herself up and dressed. The assistant left the room, and Helen followed shortly afterwards.

I caught up with Helen in the reception area, just after she had paid her bill and made her next appointment. At my invitation, we walked together to one of the rooms the Centre had provided for me to use for interviews. I asked her about many different aspects of this complex production, during our time together, and how she felt features within it may, or may not contribute to her therapy experience here. My descriptive scenic mapping (TFM schedule) and the observation I had just conducted with her both guided and underpinned our conversation, as we explored the nuances within this. I learned further insight into the whole essence of her experience here, in terms of the therapy she receives and how she engages 
with this therapy production. An example of her scripted narrative is below, highlighting what, for her, is the essence of this treatment.

Helen's narrative:

I have been coming here for many years. They straighten me out, and put me back in order, so I always come back here. I mainly have problems with sciatica down my leg and tension in my shoulders. I come around once every 6-8 weeks, although if it needs a bit extra, I come 1 or 2 times a week. He pulls it back in, cracks my spine in various places, gives me these 'big hugs' [spinal adjustments] and manipulates my right shoulder. Sometimes he [practitioner] tells me one leg is shorter than the other. You always get the manipulation, followed by massage and electrotherapy - they knock you about, massage you and then leave you to recover! I come here because I need the treatment, not because I want it. If I have a cold, they sometimes put some menthol in the massage cream. I've always been very satisfied, both when it was the former [now retired] owner, and how it is now. I like it here and I like the people.

They have a pleasant, plain reception area, and all the rooms are basically the same, with just plain walls and everything in it that practitioners' need. I've not really given any thought to the internal setting as it hasn't changed in all the time I have been coming here, but I think it is ideal for this type of therapy. There is a car park not too far away, so that's good. I've never considered whether the clinic should be 'glammed up', and I wouldn't expect a place like this to be like a beauty parlour. Where I go to get my nails done, they have soft lighting, sweet music, little oils, heated treatment beds and colour co-ordinated linens. They make you feel special, it's relaxing. It's a different environment for a different treatment. I wouldn't expect them to do that here, it's not that kind of place. You come here for specific problems. Here they keep the massage cream in a big tub - you wouldn't get that at the beauty salon! I suppose some meditation type music may enhance relaxation during treatment here, although in a short session that would leave no time to chat. Fragrant oils and music are just not important in a place like this, and if they made it prettier, more luxurious that may put the prices up, which I wouldn't want!

This narrative reveals a number of issues with value to engaging and understanding Helen's whole therapy experience, within the setting that created it. Appearing a most functional event, Helen's expectations and experiences here, and the place or backdrop where this all 
happens, all exhibit a clinical and functional intention that is not unlike mainstream medical care. From the entrance reception, which presented as somewhere to 'wait' for things to happen, rather than a place where something could happen itself, a lack of interest and content in aesthetics or style made 'functionality' almost the focus, by default.
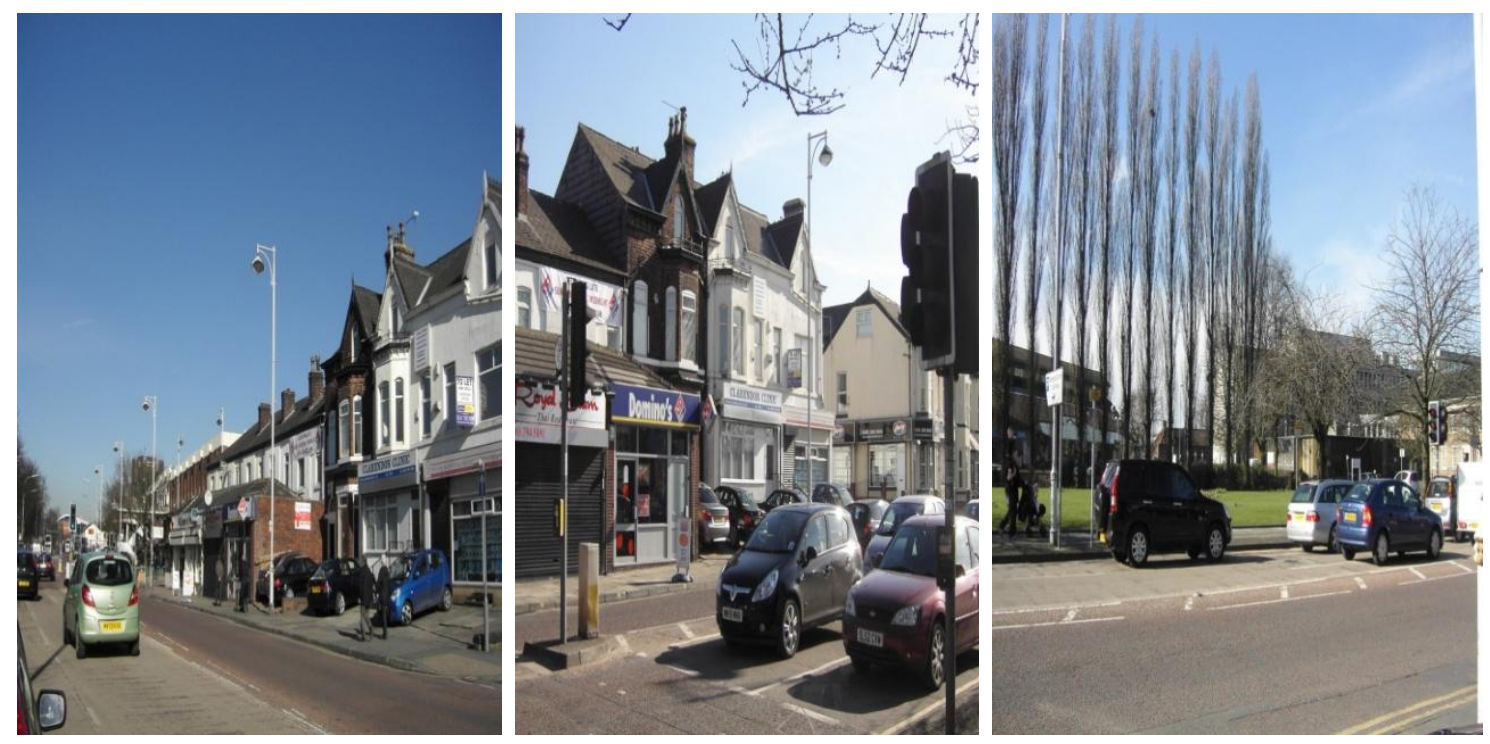

Triptych 6.1: The clinic exterior

The fact that this clinic building is functional, the treatment is functional, the expectations of patients are functional and their outcomes are functional is in keeping with the model promoted by the Institute where I, and a number of practitioners here, trained (including wearing white clinical tunics and the choice of navy blue and white towels). Underpinning their performance, this approach teaches therapists to view themselves as 'medical' practitioners and therapy as a 'medical' treatment; different from perceptions of massage as a relaxing/superficial therapy.

I wasn't surprised to see this, however I was surprised to see how big the gulf is between individual practitioners and between different presentations of therapies, and how much I have deviated from the model I was trained in since completing an undergraduate education in CAM and learning a different perspective (my continuing professional development). Consideration of dynamic or vital healing was never part of this original equation and is something that I now endeavour to deliver to patients; even though the approach I use to underpin this is broadly similar to those in this setting.

Bringing my perspective into this setting, I struggled to see why these characters should limit the potential' of their therapy, in this way. From my education and understanding of 'healing' 
thera pies, I had learned that the potential exists, in theory at least, for all thera py outcomes to be either functional or more than functional, depending upon the hands that promote them. Just 15 minutes down the road from my own clinic, but I could have been a million miles away in terms of the potential that is applied. The model used here made me question the ways 'CAM' is used and understood by different people, and how people can occupy different positions in regard to this (I am holistic, so more a soft/interpretivist science while here is more functional, hard/positivist science) may influence both the care patients expect and practitioners deliver. As a long time patient here, I am unclear whether Helen held or accepted her functional expectations of therapy before first coming, or whether these have become her expectations over time. However, she seems to judge success on how she feels and functions physically, and so keeps coming back. She is satisfied with this approach because she comes here to get 'straightened out', not to 'relax' or feel 'special'. This is a different environment for a different treatment.

Listening to Helen, I realised that despite on the surface delivering the same therapies (I am also a practitioner of Remedial Massage), this world of CAM was a different world to the one I ordinarily belong to. In my world of CAM, the aim is always to create the conditions where the body may 'heal itself' aided by certain bodywork techniques. I do not only put patients 'back in order'. A natural part of this is feeling relaxed and comfortable in the treatment space (soft lighting, nice linens), as is my choice not to dress in clinical attire (and therefore not 'look' like a medical practitioner). Therapy chatter too, may sometimes include DIY or practical topics but when I engage in these it is with a focus on creating a holistic understanding of how the patient has come to need my services and devise opportunities to progress. It is also a way of entering my patient's world and building a genuine, caring rapport with them. The approach I witnessed here seemed to have a different quality and intent to that one.

The difference may be that the practitioners here have typically followed a different trajectory to me (they have not deviated from the original doctrines) so their ethos has remained aligned closer to biomedicine (even emulating biomedicine?), with the primary aim of functional rel ief from physical problems. I felt this may explain also why the place and people present as functional.

A theme I have found equally dominant across many of the research conferences I attended prior to commencing this study, is one of researchers often seeking to emulate biomedicine in their approach to CAM research and apply homogenous interventions for problems rather than 
heterogeneous therapies for people. Supported by this ethos and by 'satisfactory' results, it is plain that these practitioners and the researchers at such events simply operate differently to me and so see little need for recognition or belief in much potential beyond gaining physical relief. As in therapy, I recognise this as determining the limits of potential outcomes. This is not a reflection of the people, but of the ways they appear to work. Indeed I'm sure I would feel trapped and impotent if I ever had to revert back to this model again.

Challenging my understandings of CAM therapy, and how others may view this differently, has been quite uncomfortable. I had to both recognise and then contextualise my own feelings (or bias - we all have this, we just don't always admit it) in order to understand my engagement with this 'whole story'. Such recognition has been both nuanced and highly consequential. In terms of directing my own performance, this is the reason I wrote in my observation that "The content suggested a history to their relationship, but I failed to spot any real empathy or warmth within this (like the general staging and feel of this place)" - because the model of empathy and warmth that I enact is a different model to here.

I entered this site believing I shared similar characteristics to these participants (a CAM practitioner, similar training, shared locations/culture etc) and this would be my unique angle to gaining deeper data. True, without being a CAM practitioner, I would have never gained access to this setting (the senior practitioners did actually tell me this before I began on site), however, once I was here I actually found some real differences which served to create more distance between us and, through this, a deeper understanding. I feel I have really learned something valuable in 'seeing' this, although I am unsure if anyone else involved at the time was aware of this impact in their performance (i.e. how that may be viewed or interpreted by outsiders, including me).

These two key points, the use and understanding of therapy and the ethos of therapy practice, raise important questions about researching CAM. Perhaps the most critical issue is who is posing what questions, and to whom? More than a phenomenology of multiple realities, it is about the paradigm a researcher bring into their research with them and how this matches or mismatches the participant's perspectives, influencing all of the relationships they make (with people, the topic and the data). I said, of my encounter with Helen, how functional everything was. My (holistic) paradigm and the mismatch between the expectations of therapy this created in me and the 'reality' I witnessed here (revealed by a subjective engagement with the data) has likely emphasised this aspect, influencing the ways I perceived Helen's expectations and experience and making this possibility more visible. Such a response is suggested both 
because of her language, actions and outcomes and because this is different from what I expect, deliver and receive in my own therapy.
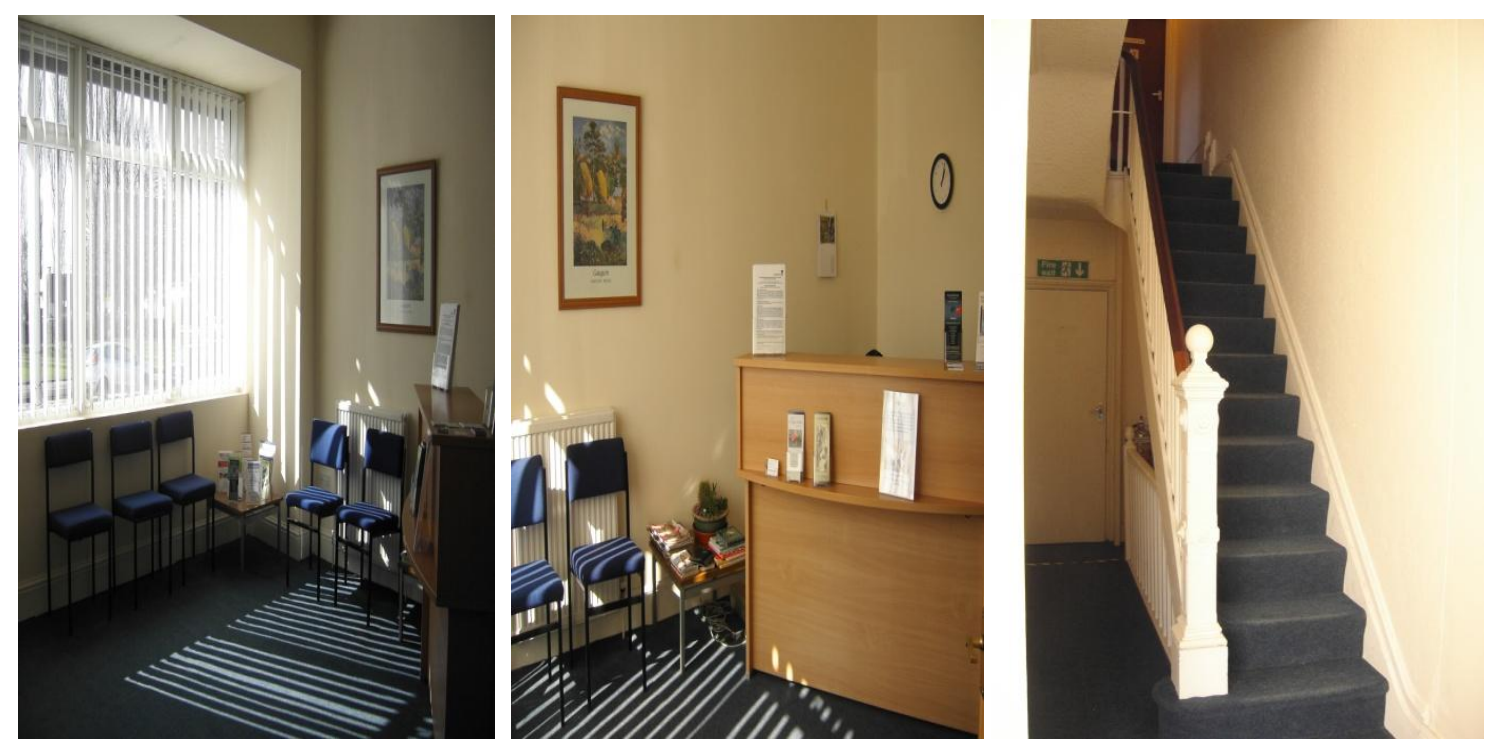

Triptych 6.2: The large reception and waiting area

This reveals a unique complexity and both my data collection and analysis. In an objective sense, Helen's paradigm (however she defines this), seems to create expectation of only a functional effect from treatment, which this staging and performance fits. There is, therefore, nothing for her to note about the mode or intention of care here, which seems to also impact the ways she engages with these events and also the potential for benefit from any wider influences. Limiting her expectations to linear cause and effect (functional) relationships means Helen would not want to pay more for 'incidental extras' here, attributing little impact within these acts. She is not here to get her nails done: this is a different environment for a different treatment.

This compartmentalised discourse means she does not consider that 'pleasant' staging could have any positive impact on 'physical' therapy. Any researcher who shares Helen's views regarding 'cause and effect' may engage with this data similarly, based upon the match between their paradigm, expectations and the reality of care here (for example the reception space may not be described as 'still and stark'). Engaging holistically, as I have, has therefore created the potential for different relationships with 'space' which I hope may open up debate on this matter (much as Helen feels 'relaxed' and 'special' where the space and intent of the people creates the potential for this). 
Here I saw how putting the people and place into the background actively allowed the 'mechanical' acts of therapy to move into the foreground (the spotlight) where patients then engage and experience them. Revealing that my paradigm and subjective values influenced my whole engagement, with the model of CAM delivered here, has highlighted potential differences between myself and these characters, impacting both what I saw and reported.

Many of those directly involved here, including Helen, feel this place includes everything that is 'essential' for their outcomes, but nothing that is not, and have no issue with this. Seeing things 'differently', I am also curious whether Helen would (or could) have gained more than just being 'straightened out', if the practitioner had intended and therefore modelled his therapy approach on achieving this? Or if Helen's expectations and satisfaction may have been any different (greater or less) if the clinic was more 'glammed up', as she put it? I cannot, obviously, answer these questions now, but it is clear that I was the only one asking. Helen, nor any other patient, ever seemed to consider such things.

\subsection{Act 1, Scene 2: The power of the people}

Presenting a different story, and with this different potential for research and therapy engagement, I want to introduce Pete (not real name). Pete is another 'regular' patient whom I met part way through my time on site. A younger man, very tall and quite shy, he also agreed easily to participate following a brief introduction (I wondered if he was so grateful for everything this Centre had done for him, he felt it was the least he could do?). I did not have the opportunity to complete a treatment observation with Pete (as I had Helen), however he did consent to provide me with an interview, that I have again scripted reflecting the essence of his experience.

Pete's narrative:

I have been coming for around 2 years. When I came, I suffered with sciatica, pains in my chest, pains in my ribs, pains in my upper back, pains in my lower back - everything you can think of! I have used the whole range of therapies here: massage, acupuncture, manipulation and electrotherapy. I had never had acupuncture before coming here. Initially the treatment was intensive, but now it is more of an MOT. I come here to get fixed, and they do that very well. The first time I came, I got a really good in-depth consultation, which set me at ease, and each time I return, they ask about my symptoms and listen to me. The whole approach is really in-depth and really thorough, I know what to expect now and I often get the same practitioner. All my 
expectations are met here, and the people really make you feel 'at home'. We have good conversations, which can take your mind off the treatment. They all seem like part of a big family, and happy to be here. All the staff are brilliant at what they do.

I come here on the motorway, and drive around 15 miles to get here. I like the old architecture and design here, but I don't pay much attention to the surroundings as I don't come here for the environment, just the treatment. The clinic is quite easy to get to, because it's not in the City centre. There is also free parking across the street, which helps with the cost of coming for treatment. It may look like a Doctor's or a Dentist's surgery, but that doesn't matter, because it's friendly. This is just an old house, plain and simple. The rooms here are all plain, and there's nothing special about them. They serve a purpose. The acupuncture room is a bit more interesting. It has a different smell, is warmer, has soft lighting and lots of 'bits and pieces'. I think that room, and the treatment, work well together. Sometimes he [practitioner] plays music, which makes you think you're not in a clinic. That's a good thing. I don't think the simpleness of the other rooms negatively impacts the treatment. When I am having a treatment, I can't see anything anyway - I am either looking at the floor, through the face-hole, or else looking up at the ceiling.

Decoration is not as important as the welcome or the treatment. I don't mind the plain rooms, because I know I'm going to get a good treatment, whether it is just the practitioner, or one of his assistants sharing the treatment. The receptionist is always nice too, she puts you at ease and the practitioners put you at ease. I think treatment is easier when you are relaxed and I feel relaxed when I come here. In previous clinics I always felt anxious or fearful of painful treatments. Waiting rooms are everywhere, some have charts, some certificates, it doesn't really matter. The pictures here don't really have any impact on me - they are not familiar scenes. This is not the most professional setting, but I don't rate those upmarket 'fancy clinics'. Those settings don't really 'cut the mustard'. It doesn't matter what a place looks like if the people there aren't any good! Their environment can make you think you'll get a better treatment, but that's not always the case. They spin you a yarn and then don't come up with the goods. They just want your money. I get exceptional service here. The whole experience is fantastic, good value, very pleasant and very nice.

Shining through Pete's account, just like Helen's, is the clear satisfaction with the results he gets from therapy here. Attending from primarily physical complaints (characterised by lots of 
pains) he credits his responses to the functional application of techniques by his practitioners, rating their skills and attention to him and what they do as 'exceptional'. This view, for Pete, comes after achieving unsatisfactory outcomes from therapy centres that were also concerned with 'looking fancy' and 'upmarket'. I could sense Pete's disappointment in those places that, with all their implied promises, failed to deliver what he wanted/needed. This seemed to have actively damaged his willingness to engage the wider potential of future settings (limited though I found this one to be) almost making him place his faith more those aspects that can be measured objectively rather than subjectively (i.e. the concrete application of techniques). In either setting, however, it is notable that Pete placed the practitioners at the centre of their acts (i.e. it was the therapists who were or weren't 'any good').

For a different reason than Helen (who had never considered if the place and people within 'this sort of treatment' would have any complementary effect, so didn't look for it), the lack of any creative staging here was of no detriment to Pete either. He had learned previously that 'fancy places' are no substitute for good therapy and so his experiences (rather than his paradigm) had taught him to disconnect provision from the place and prioritise his physical outcomes. And good therapy, with successful physical outcomes, appears to be what he gets here. The practitioners are all highly skilled (and prize their skills above all else - hence the lack of 'investment' in these surroundings) and committed to their patients, as was evidenced by many across the sample. Indeed, with the lack of much else to talk about in terms of the setting or style of this provision, the practitioners and their individual 'acts' literally take centre stage.

As I see it, developing this view of therapies and therapists says as much about prevailing discourse as it does the individual application of therapies. For Pete (and Helen), the prevailing view (or intellectual space) they bring into these interactions has the impact of actively limiting the potential outcomes in the setting, just as my prior experiences influence my outcomes whether I am the therapist or the patient. Where the settings may be different, the underlying discourse is largely the same (reflecting the nature of my local provision) and so I too come to place most value in treatments on the individual therapist, with everything else positioned around that. Being a CAM practitioner, myself, may further compound this as I know how my practice differs from other therapists, so consider it unavoidable for an individual practitioner to make a significant difference to both the experience and outcomes of therapy for their individual patients. In my specific therapy, my individual skills and hands are central factors in my own work, and while I have found that where I deliver this can add to or limit what I can 
achieve there, there is no Remedial Massage at all without me. In fact, through working and delivering therapy in a variety of places, over the years, I have learned that (particularly where the place is lacking in what I wanted or needed) the individual skills and/or knowledge of the therapist can make the difference in better or worse outcomes.
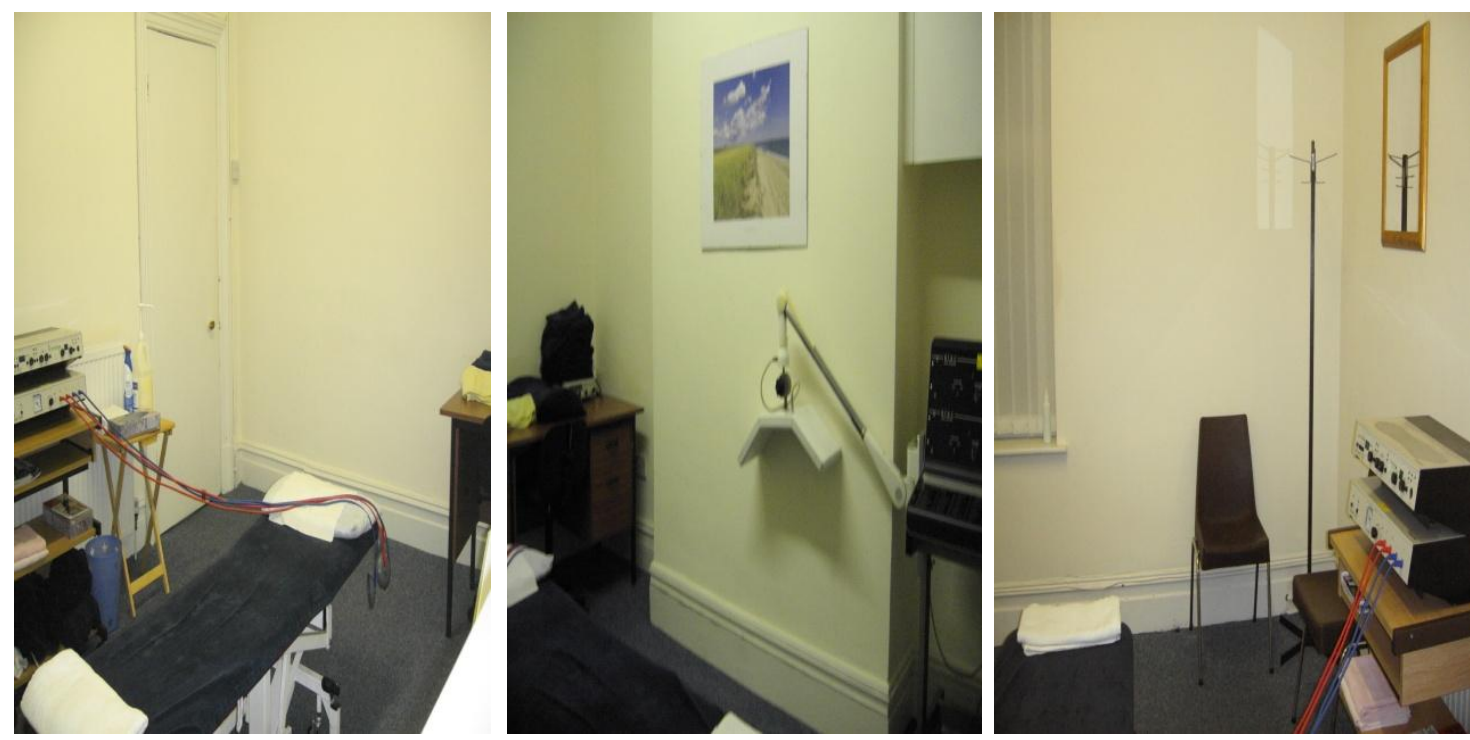

Triptych 6.3: Massage and Manipulative Therapy Room 4

Within my 'CAM discourse' this is an easy association to make, however from an academic perspective is rather more uneasy (indeed throughout, I have found blending my discourse with an academic lens to be a challenge). The issue of whether CAM practitioners can be good CAM researchers is one already of some debate (Ernst, 2010b; Lewith, 2008) with no firm conclusions and few authentic examples (as noted in Berlin, many find their holistic principles suffer when conducting research). Holding an education in many different CAM therapies, I feel to have an understanding about those outcomes that may be more dependent on the practitioner and also what outcomes may be 'reasonable', from different therapies; many researchers many not and so find my conclusions difficult to repeat.

Exploring this role of discourse (something I have only learned to do recently, since reflecting on my experiences and data from all study sites) suggests just how pervasive both any previous experiences and societal discourse can be 'in the moments' of producing data, or capturing data and eventually analysing data. For Pete and Helen (and indeed many others here), such observations do not devalue their sentiments or experiences with specific practitioners or their unique treatments. Rather I find it elevates and recognises the different complexities that lie (hidden) behind these associations in a way previous research has not. For all of us here, in this moment, I found these relationships matter; this is demonstrated 
both in Pete's claims about therapists here "doing that very well" and at previous clinics "not coming up with the goods". Equally, this is demonstrated in my choice to highlight this factor.

The default option for studies seems to require disconnecting the people from their practice, so that 'interventions' can be evaluated. But Pete's account, along with others here, makes me question whether this is even a valid approach? Without exception each of these practitioners regard themselves as 'active' within the therapies they deliver and the relationships they build, something that I also recognise from my own work, so maybe the question should be not how or where should the two be separated but whether the two should be separated at all?

Either confirming this is a phenomenon of note, or the current tensions within addressing this, I spent a long time noting and describing the relationships between these people and their acts (mine included). I had some awareness at the start, but I only really connected this with myself when I finally created the 'space' to see how my whole performance fitted together with those of my participants. This has been has been an important and revealing step in exposing/exploring the value of different 'specific' and 'non-specific' components within these whole therapy performances, and in learning how best to connect the parts.

\subsection{Act 1, Scene 3: The space is not enough}

I began this study believing that the place, the service, and how CAM is provided is ultimately the responsibility of practitioners (just as I believed the direction, execution and presentation of my research was within my responsibility). Through my engagement here, however, I am learning this may not entirely be the case, as beyond the gaze of many patients there are both personal and societal forces that influence the practitioner and patient experience.

Simon (not real name) is a senior partner in the Centre and has worked here for a number of years, first as a junior and then a senior practitioner. Responsible not only for general staffing and the smooth running of the Centre, Simon also handles a busy patient workload which he shares with another senior practitioner and a small team of therapy assistants in a model of care that is regarded unique to this Centre (developed and handed down to this therapy team by the 'former' senior practitioner, and current landlord).

I observed Simon on numerous occasions during my time on site, sometimes formally and other times informally. My general impression of him was as a warm, caring and dedicated character with a quiet authority and something of a 'father figure' to the younger or less experienced therapists. Together with another senior therapist, Simon sets the tone for the 
whole practice in terms of dress and conduct, and with regard to some treatment protocols. As a team, these characters make a conscious effort to create good staff rapport, and believe that the working atmosphere in this clinic (whether positive or negative) has the potential to impact both staff relationships and patient experiences. Strategies employed to support a positive atmosphere, include provision of an informal space (the main office/consultation suite/staff room) where practitioners can relax and be more 'playful' allowing them to be focussed on the patient in the treatment spaces, and annual social evening/staff meal where clinic hierarchies can be set aside, if only briefly.

It was during his interview that I got to know more about the man behind these methods. Conducted in two segments, on separate days (after our initial conversation was interrupted by an 'emergency' patient arrival; not ideal but Simon's clear dedication meant such things could almost be expected) Simon shared with me much about his past, present and future hopes that seemed to suggest both active and passive roles in his enactment of therapy which challenged the idea that his practice, while independent, was also autonomous.

Simon's narrative:

My previous career was in sales and banking, which I found unfulfilling - it was like being 'locked up'. I came here first as a patient, for a back and neck problem, and was stimulated to train in Osteopathy. The [former] owner recommended me to a training course in remedial massage first, and after I qualified, I came to work here as a selfemployed therapist - that was 25 years ago. I have also trained in advanced massage and osteopathy, and studied privately with two established practitioners (one a Chiropractor/Osteopath, the other a Naturopath). I mainly use soft tissue massage, manipulations, electrotherapy and magnetic field therapy. The protocol here is not one I was trained in, but one that was developed within the practice, by the former owner. We keep it because it is practical and it works. It also means we can see a high volume of patients in a day, which helps us to meet the demand we have here and keep the fees lower. We only ever raise these when it is unavoidable, not periodically like some places. I will treat any condition I feel may respond to this therapy.

I do use some conventional diagnostics in my work (e.g. MRI scans), but I still align myself and what I do more with holistic or CAM therapies. I try to look at my patients holistically, and understand the cause of their complaints, but at the outset, they often just want relief from their pain, so we do this first, and then try to address other 
lifestyle factors later. Sometimes, I feel like the public 'expect' me to make them better. I try to do this, wherever possible, but if my approach is not working I will always refer people on. I never abandon a patient. I am in this profession to help people, just as I was helped. I don't always practise what I preach though - I'm not that good! I believe the person who delivers treatment makes a difference to that treatment, and I cannot divorce myself from the treatments I deliver. The quality of outcomes will always be related to me. Sometimes patients will have a preferred practitioner. I also cross-refer within the practice. I enjoy 'team working' and between us, we pretty much do whatever needs doing.

I try not to think too much about the impact of setting on therapy. I have no power to change it. Really this setting is far from ideal! I inherited it when the former owner retired. I have looked for alternatives, within this locality, but nothing would be any better. The building itself is rented, old and expensive to keep. Our overheads are quite high. The nearby parking provision is currently adequate, but that situation is beyond our control and could always change. The room sizes are mostly okay, but it is not all ground floor which can make access an issue. We do the majority of first consults in the large office, and then first treatments in Room 2. That's why most of our anatomical posters are in there. Internally, the building has been modified a number of times over the last 25 years, and I couldn't foresee any further material changes [limitations of the building]. It's currently in its best state ever! Ideally, I would like all ground floor, light airy rooms where I could choose the decor and surfaces, with nice vinyl flooring (easy maintenance), colour themed rooms, on site car parking and a separate practitioner bathroom [currently a shared one with patients]. The way this is, it determines what we can do here. I don't like the lighting either, but it is good for working. Like everything here, this is serviceable! I don't know if this environment has any direct effect on patients, but I know it has a direct effect on me. It would be much easier to do my work in a more 'ideal' clinic.

I am happy with the general location of the clinic, but I would prefer to work from a better building. I think a nicer environment would benefit the practitioners, making them more relaxed, a better flow. Better access would also benefit patients, they make like the space better, be more comfortable. However, I am rather loathe to move, as the clinic is so established [been here for over 30 years]. We run 3 treatment rooms here, so we can make sure there is always one available on the ground floor for people 
who can't make the stairs. Each room is private, which means we can have free and open conversations with patients. I used to work in a place with curtains, and found this inhibited conversation. Most essential, for my work, is a hydraulic treatment couch. Since I got one of these, my whole delivery improved. Fixed height couches restrict application as they don't take into account different sizes of patients or different heights of practitioners, and I think people often feel more precarious on a fixed height couch.

Throughout Simon's narrative I found a recurrent theme of pragmatic realism, accepting but not really satisfied with either this location or context for his work and a lack of potential to improve on this. Presenting some conflict for this character, he almost 'walks a tightrope' between two worlds - the one he wants to belong to (mainstream, accepted) and one where his heart lies (alternative, marginalised). In reality, he does not fully belong to either and has likely compromised much to achieve what he already has.

Despite this, he practically bounds with energy and enthusiasm for his therapy approach, which has proven effective for both himself and his patients. He tackles every case, every problem, in as 'complete' a way as he can (thus reflecting both his natural vocation and the practitioner training he has completed, first as a Remedial Massage Therapist and then a 'democratic' Osteopath), however he is equally challenged because many patients simply seek a 'quick fix' for their problems. He feels compelled to try and provide this (hoping to generate more holistic outcomes in the longer term - although I never saw evidence of him achieving this) which I felt further affected him.

In this respect, the nature of the setting does not easily support Simon, which impacts the experience for himself and that he gives to his patients. This is something he feels powerless to change, although Simon is not really powerless. In fact, he is the senior partner in a successful private practice, with many years of clinical experience behind him and all the authority he needs to both create and enact therapy however he sees fit. Yet somehow, within these circumstances, he does not seem to be the master of his own actions and despite having a 'vision' for his ideal practice, he does not pursue this; rather he fears the potential (financial and professional) repercussions of introducing any changes that are not 'evidence based' and this actively seems to limit him. 

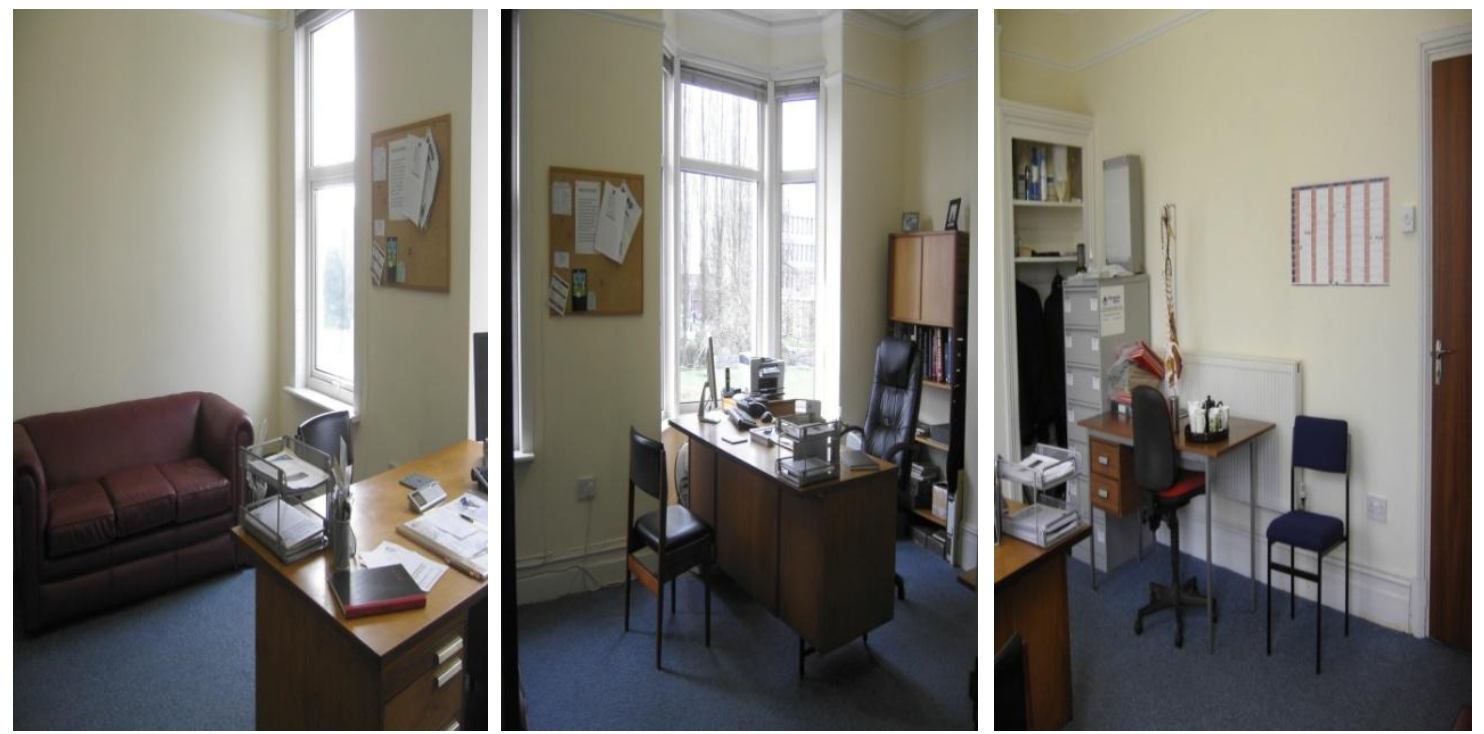

Triptych 6.4: Main Office/Consultation Suite/Staff Room

One day, he actually confided to me that one of his reasons for taking part in the study was to see if I may generate findings to support (or evidence the value of) making improvements to this practice. Reflecting on this, I began to sense that this 'space' was creating (for Simon) a restricted therapy practice, which Simon and others then deliver to these patients. This raises an interesting dynamic that I felt Simon was aware of, but perhaps did not appreciate the full extent of.

Rather a pathetic about the whole situation, he chooses to soldier on the best he can and 'tries not to think about it too much' because patients need his service (this, in itself, suggests that if he did think about it he may have to change things). In fact, he seems to work even harder to make up for the limits imposed by this setting, concerned that patient outcomes shouldn't suffer for the benefits of this location and established 'reputation' (although I am unclear who actually benefits and to what degree). As an onlooker, I began to question whether his 'reducing focus' to meet the practical needs of therapy (such as private rooms, hydraulic therapy couches, lighting and heating to support physical outcomes) with less concern for wider aesthetics, was a sign of his failing will - having started out idealistic but then been confronted or crushed by the reality of opposition - and so becomes a positive readjustment to meet the demands of societal discourse. As an emerging theory, this could apply just as easily to me in my clinical work, and in my research (although in both I have found that adopting functionality over freedom leads to ill health and that resistance breeds recovery).

I don't think I realised, at this early point in my study, the full implications of this observation; only that every decision, action and consequence made here has implications for how Simon 
feels about his role and delivers his treatments. Essentially, he describes himself as a 'buffer' between the context and his therapy, mediating any negative effect before it can impact the patient. Taking this idea wider, I began to wonder if this could be partly responsible for reducing the full potential of his therapy and contributing to participants' focus on their practical outcomes.

In terms of research, this could be happening here also, as I have found myself at times trying to mediate different influences and environments so as not to negatively impact (or limit the negative impact of these) on my work. In almost every case, of course, this has turned out to be a false ideal and that in absorbing these impacts within myself I have become the negative influence, with costs and consequences for everything I do. This all came to light when I entered a brief period of counselling ( found completing this thesis actually pushed me into a kind of breakdown) and I began to see how disconnected I had become (emotionally) through trying to manage the 'divide' between my ideology and the reality around me. I suppose it was my way of coping with the stresses and strains upon me. Looking again at Simon's narrative, I can see elements of this coping strategy within his performance too. This may explain his lack of confidence (or conviction) to change his practice to how he would like it to be, despite feeling this would have made a positive difference to all outcomes, and why he feels unsupported in this.

During my initial reading of this I felt saddened and cross that Simon lacked the support to be the practitioner he wants to be. His model of working is not his own and his alignment with the Democratic Osteopathic Council (DOC) meant that he had to abandon the title of 'Osteopath' when the General Osteopathic Council (GOC) took legal protection over this. Since then, his remit has been limited to Manipulative Therapy and he has come to rely on the reputation of the practice when it was under the former senior practitioner's (and current landlord's) control. The fact that many patients still regard this as "The Osteopath's" means he also has to continue re-enforcing this 'demotion in title' to maintain a legal position.

This looks to have trapped him in a model of practice that, while meeting the expectations of patients, is not his ideal. Reflecting on this later, I found my emotional response was partly because I always believed practitioners got more support, when in group practice and had often wished to find myself in such a position. Simon's story seemed to contradict this, suggesting that it is possible to be lonely, even in a crowd, if your ideals are different from others. Indeed, he does not appear to have shared his ideal visions for the Centre with either his patients or colleagues, which suggests a further loneliness. 
But Simon's position in practice is not so different from mine in research. Like Simon, I have felt that support enables authenticity as I have also felt more able to be authentic in my research when I felt supported in this. Moreover, through subsequent experiences (and personal trials) I have found my engagement and research performance threatened. This began slowly, at first, as I began sharing (and defending) my work with colleagues. Then, during final write up, I had to live through a series of family illnesses (first my father-in-law was admitted to hospital and died, and then my youngest child suffered a series of repeat hospital admissions) during which I became engulfed (I choose these words carefully, to show gravity) by a tide of unreflective and reductionist medical views that tried to force a different model of being onto my existence (treating symptom patterns only). Trying to balance this approach into a more humane one was a crushing experience - because the support was just not there.

I was a lone voice 'in this wilderness' and this fed through into my writing (at least I had completed my 'good data' by then) making my analysis and presentations start to become increasingly unreflective and reductionist. I didn't see this straight away, but did the best I could at the time (which was far short of what I had promised myself or my supervisors). They tried to support me but it was too late, I had lost my conviction. I failed to keep my head a mid the 'chaos' (if a 6 year old needs medication to get through the day, there must be something wrong with their environment) and this made it harder to succeed in my work.

These observations were not present during the original data collection and early analysis (as I hadn't lived through them then) but as part of my current lens, I feel it is important to include them now, because they are my outcomes from losing my head and adapting to suit my circumstances. Not the data I intended to pull through when I began this participation, I see this as not unlike Simon's approach now (where this limits his potential and erodes his convictions), because I have come out the other side. Simon is not there yet; he has yet to begin his fight back.

In the characters at research conferences and educational events, I have seen others like Simon too (doing their best, while losing their heads). They bury their convictions in the face of a fierce and dominant discourse (biomedicine and science/academia) and adapt to the expectations of those around them, like a self-perpetuating cycle. Simon's need for support to be authentic may therefore suggest a lack of conviction in his original alternative methods of practice or an 'erosion' of this, over time, by the discourse within this environment. 

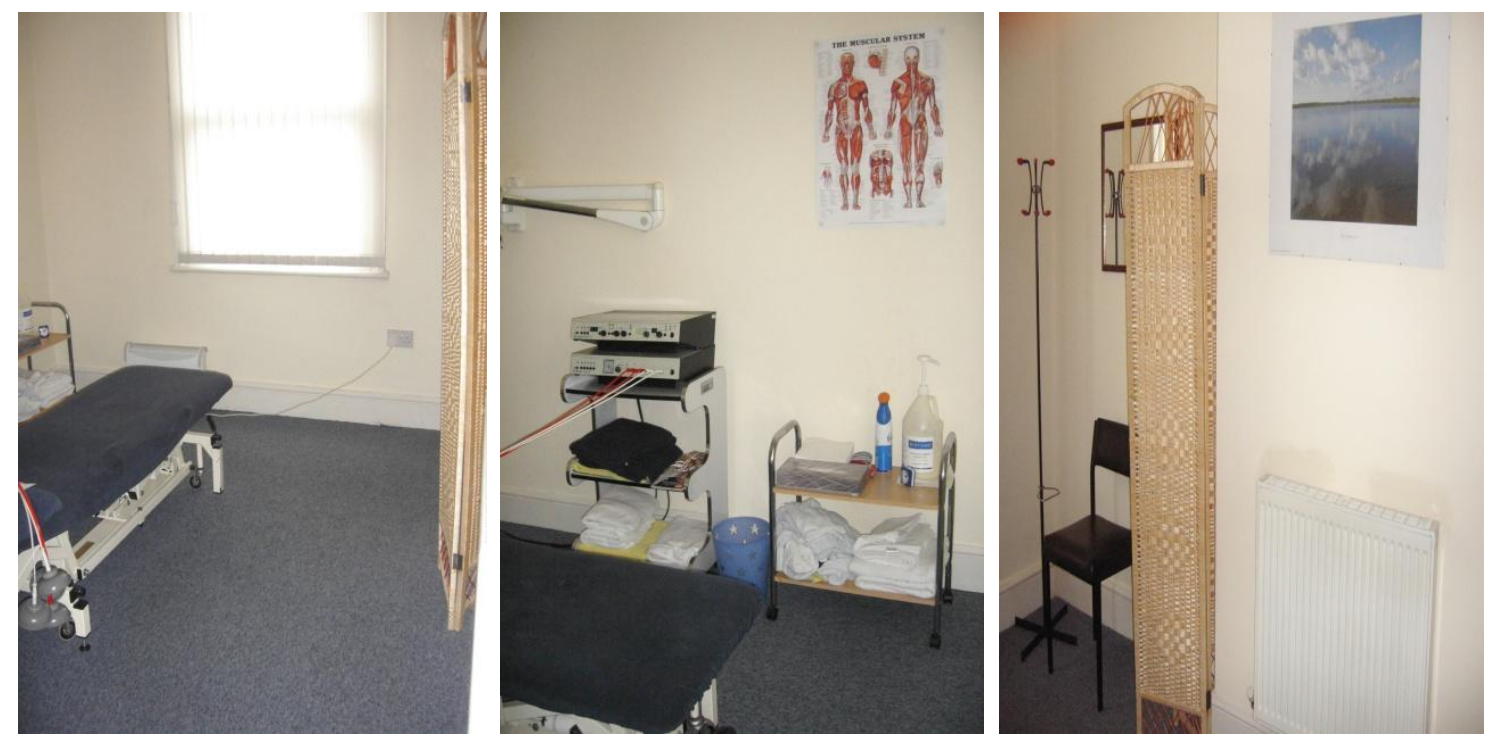

Triptych 6.5: Massage and Manipulative Therapy Room 3

At the start of my time here, I felt I would not like to work in a place as 'stark' and 'clinical' as this, however over time I began to question whether I could perhaps mediate my practice to fit in somewhere like here. This shocking admission (I typically regard myself a most convicted, even zealous CAM practitioner) was because the warmth of these characters helped me experience this setting as 'less cold' than it actually was. Indeed since completing my fieldwork here, I have referred patients into this clinic (including my husband) for treatment, if either it was more local to them or they needed manipulations (which I don't provide). I still find each time, this place feels as cold as the first, however my developing familiarity with these people, continues to make it somehow 'better'.

I suppose any degree of oppression may depend on how oppressive the environment feels for the individual and I sense that, for Simon, having learned to 'fit in' here with those around him (who, in the main, do not share his underlying holistic views) reduces this to the point where it feels 'normal' to practice this way. Again, I identify with this as my University has had a similar effect on me, made better by those around me but still eroding my (holistic) principles and desires.

Exploring the presence or lack of support that practitioners feel within their therapy, and how this may affect them and the care they deliver to patients, is something I feel is often neglected in research yet presents a real opportunity for understanding the nuances within real world practice, and real world outcomes of CAM. Failing to capture what is present may not be as simple as there being nothing present to capture, but a reflection of the willingness or ability of researchers to connect with participants' performances in a complex way. This 
may even underpin some of the differences noted between CAM trials and how 'scientific' outcomes can fail to reflect case study and anecdotal evidence.

Bringing this back to Simon, it is clear he purports to see himself as an integral part of each performance he gives (both in term of evaluation and manual manipulations) and this is something I saw within his performances also. Given this, I can only wonder why clinical research has not yet adequately addressed the value placed by Simon and others on themselves, as a part of the care they deliver. Perhaps studies will only be brave enough to change this when researchers are sufficiently supported by their environments (and convictions) to do so. My engagement beyond this study, both in conferences and events worldwide, suggests the people, if not the conditions, are ready. Like Simon, however, they may be so oppressed by the presence of Evidence Based Medicine (EBM) they cannot realise the potential outcomes of acting with conviction first (and evidence later). It was only when I eventually realised this that I became truly able to mount the challenge I had originally intended to embark upon!

This is an important issue for myself (and CAM research) going forwards, as an additional layer of complexity in practice not easily identified or acknowledged, but with the potential to influence therapy delivery and research outcomes. Looking back to here (I speak having since completed time in other field sites) I can see that all parties, including myself, operated with significantly more constraints than I found elsewhere and often without real awareness of this, in the moment of action.

\subsection{Act 1, Scene 4: A new breed of 'professionalism'}

Related to this, the power of 'professionalism' in CAM is an area that generally I find to be more promoted than critiqued, especially by newer CAM graduates and practitioners. Lydia (not real name) is one such character. Lydia works part-time as a massage assistant to Simon and also provides sports massage and 'physiotherapy led Pilates' independently here, while she completes her University degree in physiotherapy. Like me, she was a private CAM therapist first (before entering higher education) and so represents an approach to integrated medicine that may be regarded a pragmatic step to developing CAM into a credible healthcare 'alternative'.

I spent a lot of time with Lydia, while on site, and we had some great conversations both about her past, present and future aims as a therapist. I was struck by both her skills (she has strong hands) and her honesty, in regard to the potential she sees for both physiotherapy and CAM 
therapies, within current discourse, and the imposed compromises of the different contexts where she has worked.

Lydia's narrative:

I'm the type of person who needs to learn something new all the time. A friend suggested I try a massage course, and it seemed like a good idea. I studied Swedish massage and body massage. My teacher said I had a talent for therapy, and suggested I study further. My interest in sport led to sports massage training, and I am currently in the final year of a BSC physiotherapy degree. I am a part of the treatment and I try to improve myself and my therapy all the time. I recently did an accredited course in Pilates for physiotherapists. I would like to work in sports rehab. I don't think the NHS model of care is good enough. In NHS, the trend is 'don't use hands on, prescribe exercise'. Tutors who have worked in the NHS don't teach the value hands-on-therapy, while those who worked in private sports therapy encourage massage courses. I prefer a hands-on to a hands-off approach. My personal model for delivery conflicts with the NHS model, because I try to be holistic. Among my physiotherapy colleagues, I am unique in having hands-on skills and I get great feedback from patients.

I work here part-time, assisting with massage and electrotherapy. I also see my own private patients here, with sports massage and Pilates. Everyone here tries to be approachable with patients, so they feel comfortable with treatment. In Physio, you have to be confident and know what you're doing. Patients need to feel comfortable with the practitioner to have confidence in the treatment/therapy, or the treatment response will not be as good. I think that personalities affect and influence the treatment as well as influencing the relationship with patients. Good working relationships among practitioners make the treatments better and final goals more achievable. I have learned that I need to feel comfortable with the people I work with, or I will lose my confidence. You need a good environment to achieve goals and feel good with other people. Without support from seniors, therapy doesn't work as well. Where I have been on NHS placements, people stress you out all the time. The working relations in those environments are not as good as they might seem, and I think this can damage treatment delivery. The atmosphere here is always great, and [the Manipulative Practitioners] are the best bosses I have ever had. They are always laughing, smiling, never stressing other people around them. 
I like the professional image of the clinic, and I think this prevents misunderstandings about the nature of massage on offer here. I don't like working from home. For my private work I advertise on the internet, and have had many crank calls. The location here is good. There are good motorway links, and local landmarks, which makes it easy for patients to find. We provide a service to this area. The room layouts are much better than NHS settings, and we have greater privacy here. I think this is more comfortable for patients and practitioners. You have space to move all around the patient here. I use a couple of different rooms here, depending if I am doing massage or a Pilates class, and pay rent in the form of commission. The rooms are well set out, although I would like more educational posters. I think that space has a direct effect on the therapist and the setting arrangement definitely facilitates how therapy is delivered. For the Pilates class I share the attic. The space functions okay once it is cleared out. I have to move the furniture out of the way, put down exercise mats and bring in some equipment from home. I would like a better Pilates studio, but I'm just starting, so this is okay. I don't really change my massage space. My private patients complain that the lighting is too bright, when they are face up, having massage. I do turn this off in the summer, but in the winter it is too dark. At home I will use also use music, but here I don't really think about it. I worry that music here may suggest more relaxation than therapy.

I found Lydia's tale one of caution, above all else, and of trying to fit personal holistic or humanistic ideals into a therapeutic model that will be accepted and acceptable to society. Dramatised first in her approach to training and then in how she mediates her practice to fit the context she is working in, she actively presents this as being of less consequence than she knows it to be. The fact she is a very positive and pragmatic character, the kind who always sees the glass as 'half full', maintains her and her hope for a better future (for her work).

I saw a lot of my former self in Lydia, which affirmed for me how much, over the years, I have changed as a therapist and changed my ideas about therapy. In my early days as a practitioner (where Lydia is now), I likely accepted less than my ideal as the best that could be achieved, as I feel does she. The important part in this is not how different each of our definitions of holistic practice are - just that they are different - and accept this means different potential for both patient care and outcomes.

Set against Simon's narrative, I felt that Lydia mediates her intentions and delivery of care to fit her status and responsibility within this practice. She began working here as a junior 
assistant, supporting the senior practitioners in their work, and although she now also provides independent therapies (sports massage and small group Pilates classes) her performance in these additional roles continues to be influenced by her primary status as 'assistant' within the practice hierarchy. This means the clinical style of therapy she applies is less of a challenge and more compliant with the mainstream/NHS style approach adopted by the senior partners, than she would prefer. It may be that the model here, when compared to her experience in formal NHS practice, is (to Lydia at least) a more holistic alternative; still this remains a long way short of my interpretation of holism. Rather, I find this more pragmatic than holistic; something I could apply equally to others here (who may disagree with this perception).
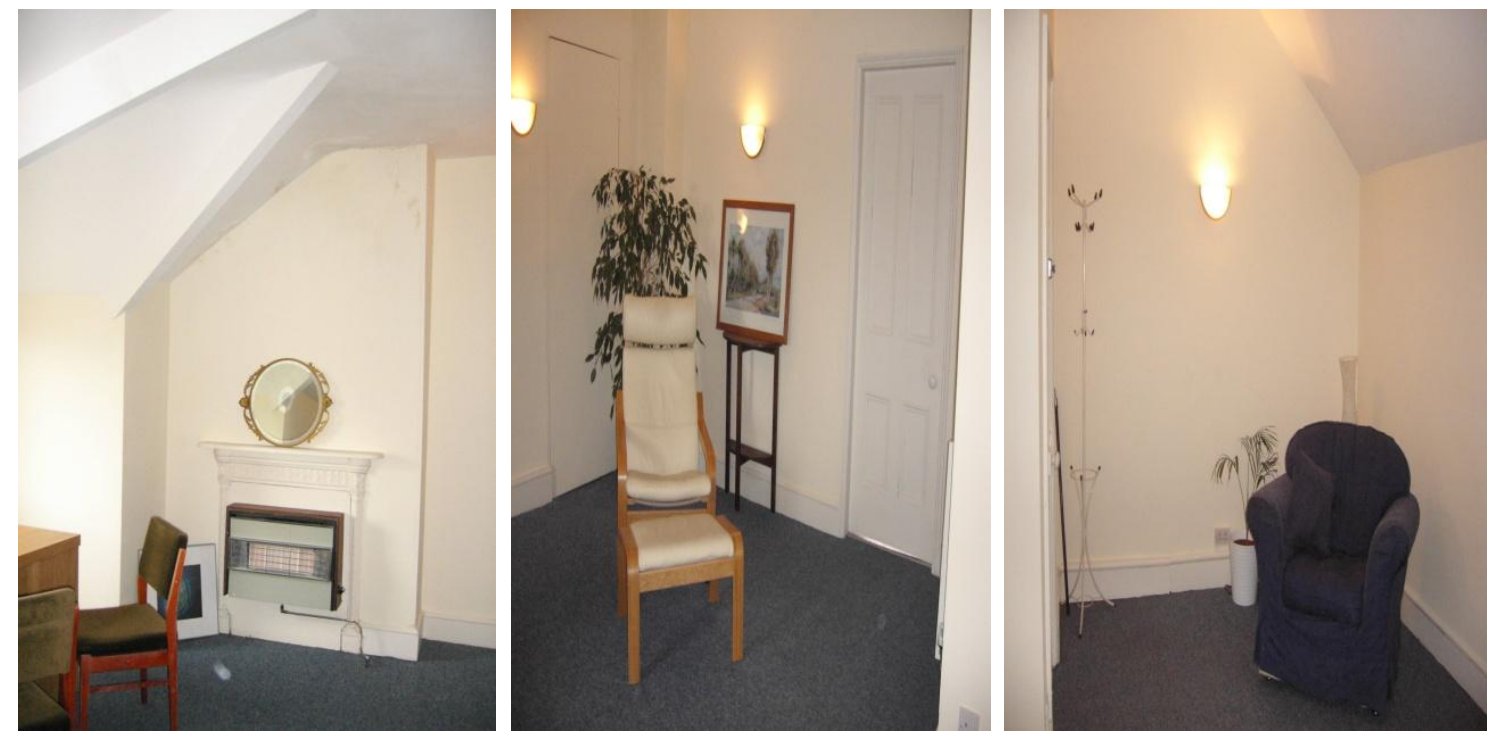

Triptych 6.6: 'Pilates' studio (Attic) and Hypnotherapy suite

Lydia does the best she can in the space and circumstances she finds herself, equally influenced by her intuition, her overarching discourse and those of the people (and place) around her. She considers this approach, while not her ideal, to manifest greater potential than when she is in NHS settings, where the people and the place can "stress you out all the time" and "damage treatment delivery".

This observation raises caution both for understanding practice and for communicating practice. When I wrote about the 'power' of this context, in my research journal at first, I spoke of how I felt this setting was too harsh and clinical, with rooms which were too COLD AND HOSTILE - no humanity in the spaces, no colour or life. I felt that I would not like to work here, clinically, at least not without modifying the space to make it softer, more human. This seemed important to me because I felt uncomfortable in such a hollow space. However, as I began to see how positively patients respond to this model, day after day, I started to question 
my commitment to my more idealised approach (i.e. soft lighting, music and aesthetics often seen as synonymous with CAMs) just as Lydia does when she is here (notably she adopts her 'ideal' more in her 'home' practice).

I was a visitor here for just 4 weeks, and began to be suppressed by this to the point where I wondered if I could get used to working in a similar environment. For Lydia, who has engaged this environment and her University/NHS placement settings, the effect seemed greater and this limited her confidence and conviction to act more authentically, even when she could. Lydia only realised the full impact of this on her, however, when I questioned her about this, suggesting the power of this reinforcement is clearly a pressure not to be underestimated. There is an irony to this, as a common characteristic of CAM is the theme of personal development, yet this reality may be viewed as inhibiting development for Lydia (and others). Unless she can actively resist this, I fear her 'development' will see her standing not alongside allied healthcare as a 'professional' equal in the future, but of being co-opted within this instead.

The position or potential afforded to Lydia, as the future of CAM healthcare, is therefore both a hope and a concern. She has the enviable position of being able to integrate practices, but currently lacks the 'space' to develop a truly holistic model. She is keen but constrained, and seems compromised by the limits of her current experiences and exposure. This is why she is concerned to avoid 'misunderstandings' about the nature of her therapies and intent on pursuing a 'professional' image more than a 'holistic' one. This provides a key insight into how different environments may affect both the individuals and practices of therapy within, and the role of this in evaluating outcomes.

\subsection{Act 1, Scene 5: Critiquing the whole (holistic) therapy performance}

As a whole package, I found this centre presents and feels like any other mainstream clinic (although others suggested a 'seaside bed and breakfast' hotel) which, for these patients, seems an acceptable arrangement. Feeling generally dated, this is no better and no worse than any other mainstream/NHS facility, although perhaps the lack of shiny, new 'sterility' helps the space to feel more 'lived in'. Keeping things 'how they are' has the added benefit of ensuring the cost of therapy does not rise to levels where this local population couldn't afford the care.

This is a busy clinic, with everything it needs to be successful; a good long-standing reputation, a prominent location and easy access with a dequate parking (nearby). Yet, in an approach to 
CAM that I came to term 'British pragmatic and functional holism', I found a clear subtext for patient expectation, care and outcomes that influenced both the delivery and ultimate potential of these therapies. There also seemed (from some within) conflict between wanting to be different and being afraid to 'look different' (for example, all dress in 'clinical' attire), like they were stuck somewhere in-between, hence the title of this chapter.

As a backdrop for outcomes, issues in mind, body and spirit here are compartmentalised; acknowledged but not integrated, with ailments addressed often in isolation from each other. Using different therapies as separate, single (rather than integrated) modalities, delivery is both prescriptive and customised, where the specific needs of individual patients are met based on their daily presentations or feedback. These therapeutic aims, alongside a clinical code of dress and utilitarian spatial backdrop support the maintenance of formal, respectful and professional relationships between practitioners and patients - creating a clear distance between the experts and the non-experts (emulating mainstream/NHS models).

Juxtaposed to these performances, there were some hidden treasures within this Centre that I feel also need to be noted, as they contained the potential for different levels of response, both in terms of acts and intentions. These were typically from practitioners whose work involves a more dynamic engagement with philosophy and with patients, for example the TCM (acupuncture and herbs) practitioner, the 'new' reflexologist (only joined the practice during my time here) and Alexander Technique teacher. Their approaches, while different in some respects, shared the common principal of creating the conditions for healing to occur, whether by balancing meridians, clearing reflex zones or awakening the senses and connecting with the inner self. These characters, unable to perform under the restrictive conditions that others accept, often brought their own 'additions' and 'tricks' into the building, to support their acts while here (for example the TCM practitioner brought softer lighting, landscape photographs and plants while the reflexologist brought candles and music).

These 'extras,' some of which were also functional, made their 'spaces' less clinical which they felt provided the potential for better healing. My observations were that this supported both the practitioners' engagement with philosophy and the patients' engagement with therapy, in a complex synergy that was perhaps most obvious by its absence throughout the rest of the Centre. These spaces (which were still quite bare) also felt much 'warmer' with the added thought and intentionality these characters put into them, although like me, these were not immune to having their Vitalistic potential diluted to 'fit' the dominant discourse and expectations of popular culture. 
Whichever guise they take, my perception of therapies here is that they are only viewed 'alternative' or 'complementary' in how they sit politically and economically, beyond the boundaries of current state-funded, mainstream healthcare provision. I never saw patients engage with these as philosophically 'alternative' or 'complementary', or understand this model as an example of 'alternative' or 'complementary' medicine. Neither did I find patients or practitioners here having much comprehension about the philosophies underpinning any approach outside their own which, while this saddened me, did not surprise me (given my previous discussions about power of discourse) and perhaps made it easier for all to accept this presentation.
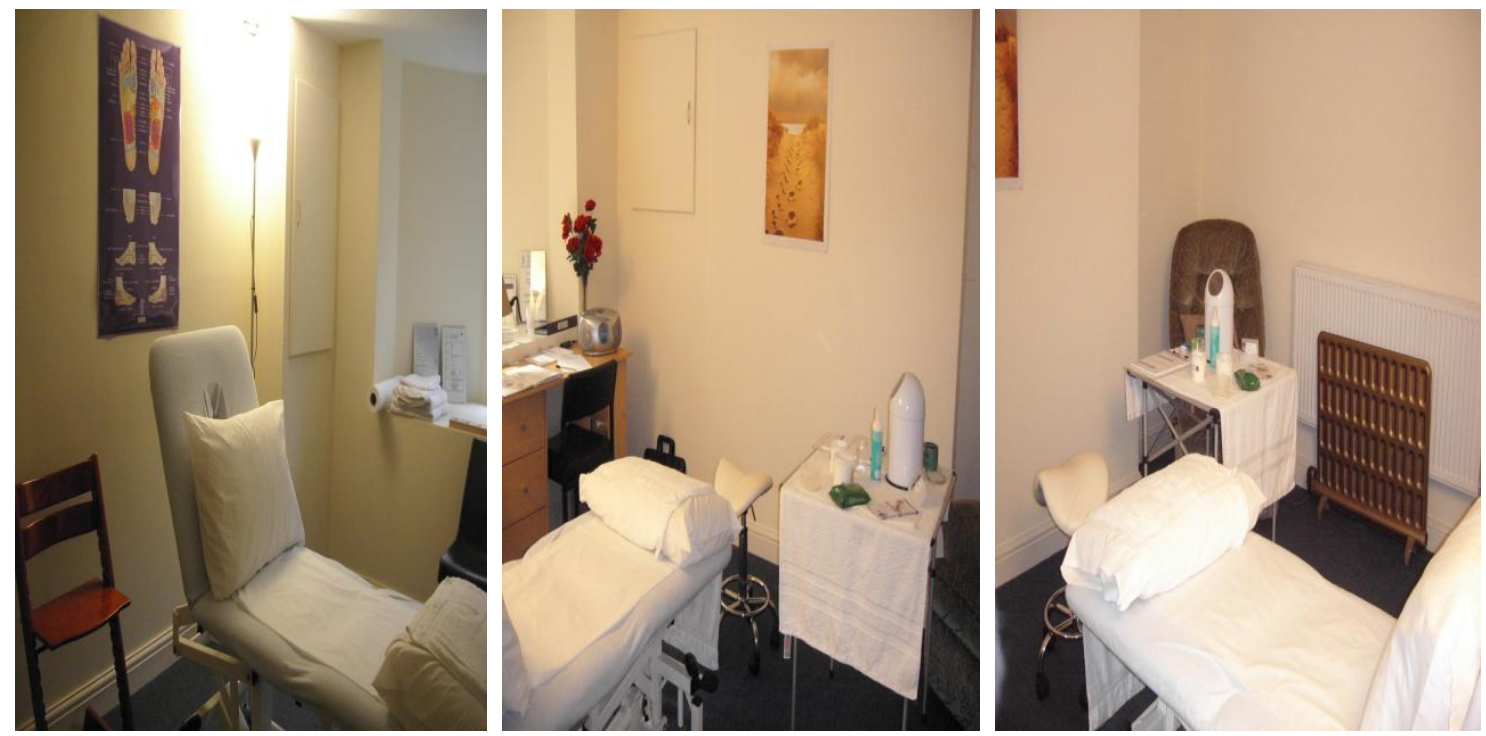

Triptych 6.7: Reflexology Room

With potential for both positive and negative impact, on the positive side patients seem to gain confidence in the quality of treatments here as a mainstream, credible practice. Practitioners too find this helps them to act with more 'professionalism' (emulating their NHS funded counterparts) which they feel prevents 'misunderstandings' about the nature of treatments and protects practitioners from being negatively 'judged', externally. On the negative side, this 'professionalism' restricts practitioners freedom to perform their roles as they may prefer, particularly those who are capable of delivering (or would like to deliver) more holistic treatments, seen from both Simon and Lydia's stories. Satisfaction, therefore, is a relative concept that depends upon the potential each individual feels exists for them, and their therapies, within their current context and dominant discourse.

Limiting practitioners in any way means patient outcomes a re also potentially limited, either in terms of what is delivered or what is achieved. Both Lydia and Simon spoke about how 'being 
supported' helps therapy to be better, but they did not mean this in just the physical sense of patient outcomes. They meant this also in terms of their confidence, to apply themselves to their full capacity. I felt this came from both the people and the place, however not all of those with the freedom to 'stage their setting' according to their convictions did so. Dramatised here, through the sharing of character stories and narratives, such effects have proved more accessible than studies of the parts alone could have revealed.

Here, I also saw how putting the people and place into the background actively allowed the 'mechanical' acts of therapy to move into the foreground (the spotlight) where patients then engage and experience them. Revealing that my paradigm and subjective values influenced my whole engagement, with the model of CAM delivered here, has highlighted potential differences between myself and these characters.
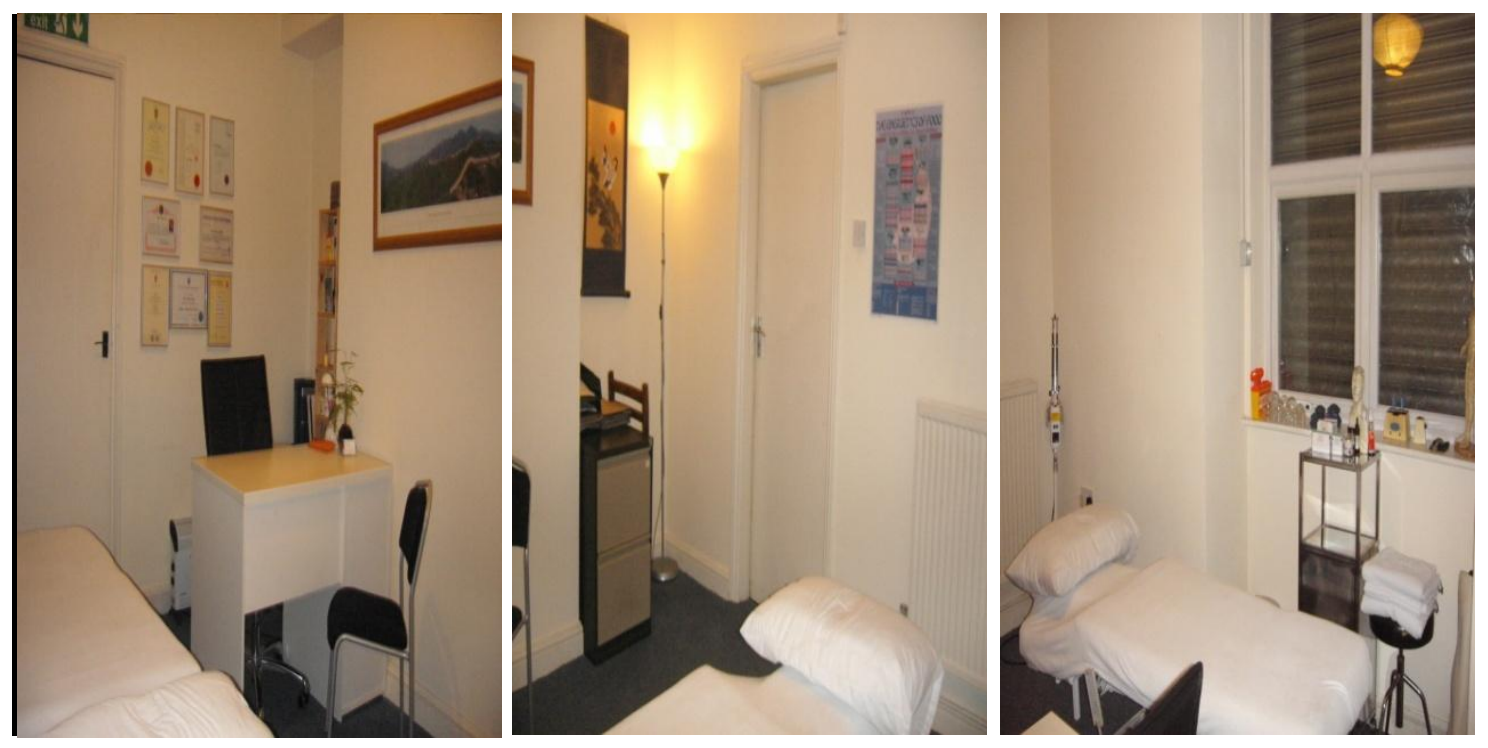

Triptych 6.8: TCM Acupuncture Room 1

I cannot conclude my discussion without also recognising the two most authentic characters I encountered here: the two receptionists. These staff, uniquely free from the pressures of 'professionalism' (that seem to affect others) are the cornerstone of the 'caring' atmosphere that helps patient experiences to be as 'pleasant' as practicable. As a small but significant ray of sunshine, they brighten up this otherwise 'cold' place. A role that did not escape any of the patients I spoke to, their easy, friendly and personable manner was integral to putting patients at ease and helping them feel relaxed immediately prior to therapy. As noted in Pete's narrative, this seemed to also help treatments to be easier while my own journal records suggest the joy and uplifting effect of these characters is almost palpable (perhaps not that hard considering there is little competition). They may be an adjunct, but have definite value. 
I said, at the start, that I felt there was a conflict here between wanting to be different and being afraid to 'look different'. Through exploring Helen's and Pete's, Simon's and Lydia's stories, I have exposed where this is so and the effects for practitioner, patients and potential therapy outcomes. A victim of its' own success, the reputation of the clinic and of these 'hard working' practitioners (past and present) has perhaps created a need for this Centre to deliver the practice model it does and for Simon (and others) to maintain that model, even though it limits or conflicts with his personal (holistic) desires. This Centre may therefore also be seen as a victim of the context and discourse that surrounds it, and the manipulation by patients dissatisfied or dismissed by conventional (NHS) medicine without adequate recovery, demanding it provide a credible and accessible alternative to supplement or replace their mainstream care.

\subsection{Act 1, Scene 6: Reflecting on my research performance}

As the first field site in the study, this was also an opportunity to refine my novel methodology and method 'on my home turf'. I felt I was on the cusp of a whole new world of discovery, travelling deeper into therapy practice than others before me, waiting to see if it was actually possible to expose and explore the presence and nature of 'complexities' in CAM. I was also excited to engage with CAM performances beyond my own and connect with other practitioners and their practices (private CAM can be a lonely business).

When I captured this data, I was viewing each episode as a kind of 'play', where character, plot and set co-created the whole event. I subjectively engaged 'in-the-moments', exposing myself to a deeper, more nuanced level of connection where it was easy to read these performances and their relationships as a whole/holistic event. I then sought to explore and analyse my responses to these acts, by identifying my participation in the culture of these performances, which has led to my revealing and evidencing a different kind of complexity than CAM research has so far revealed. This has been a challenging task as, like the data and these events, I have at times got 'bogged down' in the concrete aspects, preventing me engaging with wider relationships and placing me perilously in danger of losing my critical appreciation of the whole event. This has taught me about further effects I had not originally conceived this staging, venue and act would be capable of: namely the relationship between the phenomena, researcher and the data this produces.

Bounded and blighted by flashes of illumination and depths of despair, I have struggled to place academic objectivity and therapeutic subjectivity side-by-side with each other in this. All of my early (and some later) write-ups portrayed me as leaning too much to one side or to the 
other, never quite finding my balance. I finally recognised this as the discourses around me 'pressuring' me, which actively reduced my 'inner space' and the potential I had for exploring and exposing potential relationships (thereby threatening the entire potential of the study). In this sense, I became a reflection of the acts I had observed.

Just as Lydia had presented her struggle to fit holistic ideals into therapy practice of less consequence than it was, I had been guilty of this too. I had written myself out of the data and, in doing so, I also wrote out most of the value. Reconnecting with my inner space and turning this around has been a long, messy and 'undoing' process, but it has taught me how consequential my struggle has been to understanding these therapy practices and my research practice. So I wrote myself back in and, in an unnerving yet exciting admission, I discovered that just as these participant's models create their potential, the model I had taken into therapy observations (and later analyses) had created my potential and determined both what I expected to see and also what I did see here. I don't know if researchers, before me, simply fail to notice this or purposely omit such admissions (their final reports are often so sanitised), but I find this confirms a further transferability of Read's (2005) assertion about space creating the potential for relationships to occur, that I had not considered before. 


\section{Chapter 7 "From Holland, with Love"}

For my second setting, I travelled to mainland Europe, and worked with a large group practice in and around a historic City of North Holland. Presenting a different backdrop to the functional design and aesthetics of the UK environment, here a beauty of 'movement' dominates the centre of this City, from the medieval square to the network of canals and embankments, bridges and stunning architecture. Each time I stepped outside into this landscape my spirit 'soared'. Somehow, it felt like I had 'come home'.

Providing a whole alternative approach and conventional care (due to the prevailing legal restrictions), the model of therapy I encountered here was a complete package of Anthroposophical Medicine split across three distinct sites, each offering distinct modalities within this and patients accessing services at one or all of these sites simultaneously (generally funded by patient insurance schemes). The largest of these (Site 1) provides GP care, nurse management, anthroposophical physiotherapy, anthroposophical psychotherapy, eurythmy therapy, social care and natural dietetics), a smaller one (Site 2) offers GP care and nursing therapy (including 'enveloping'), and a third (Site 3) delivers dedicated anthroposophical physiotherapy. Across the whole practice, a total staff of seventeen practitioners (six GPs, three nurses, four physiotherapists, two psychotherapists, one eurythmy therapist and one dietician) are supported by eight assistants.

To aid a sense of the physical space, set plans are as follows:

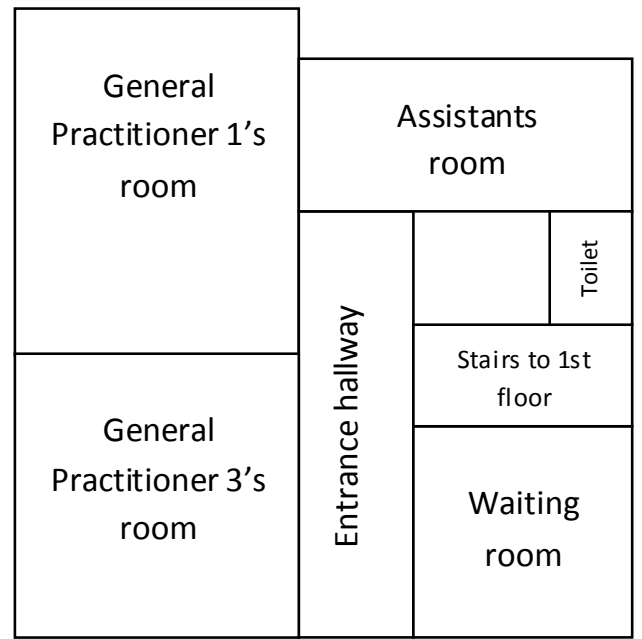

Set plan 7.1: Ground Floor at Site 1

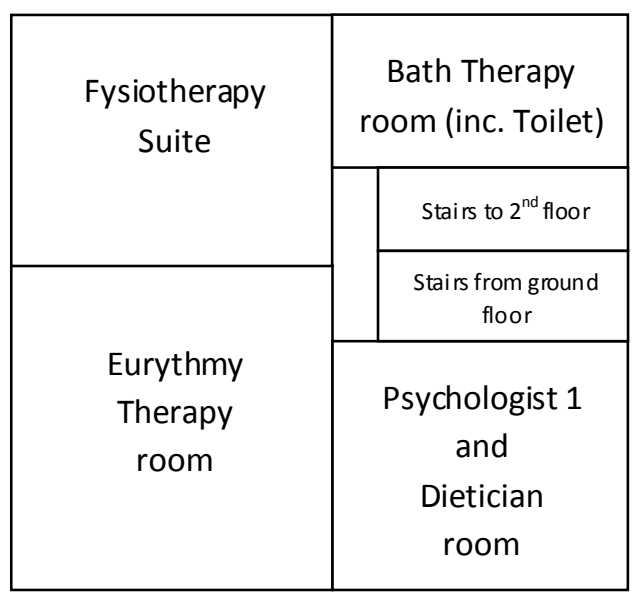

Set plan 7.2: First Floor at Site 1 


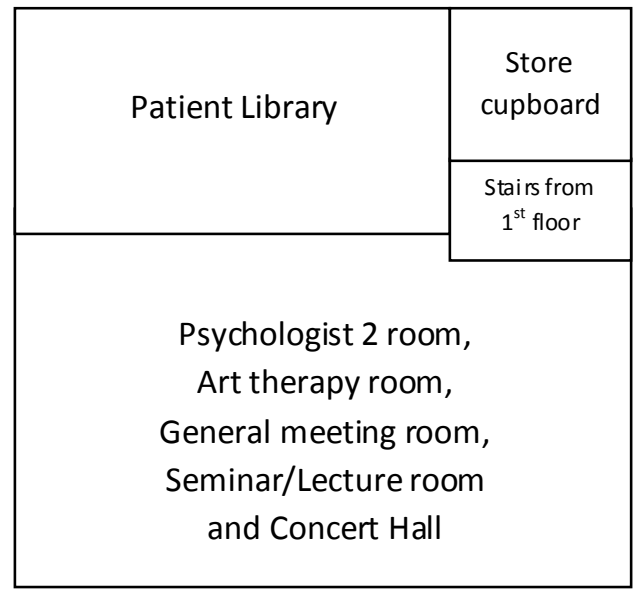

Set plan 7.3: Second Floor at Site 1

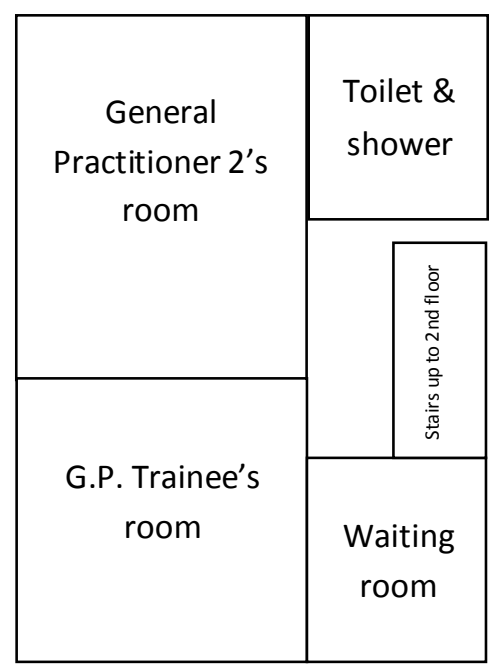

Set plan 7.5: First Floor at Site 2

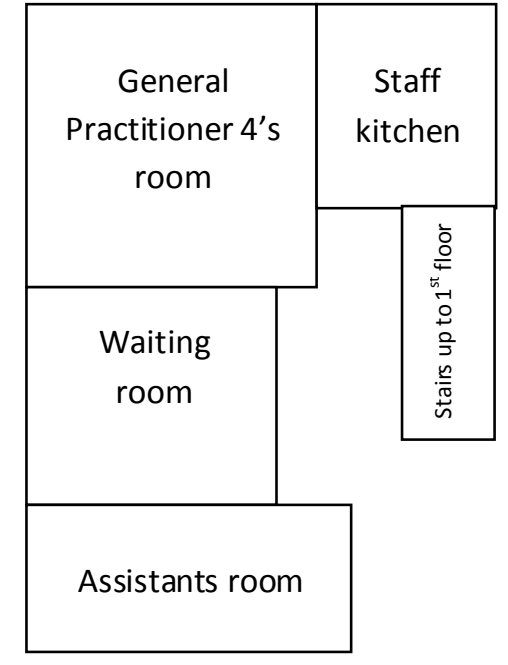

Set plan 7.4: Ground Floor at Site 2

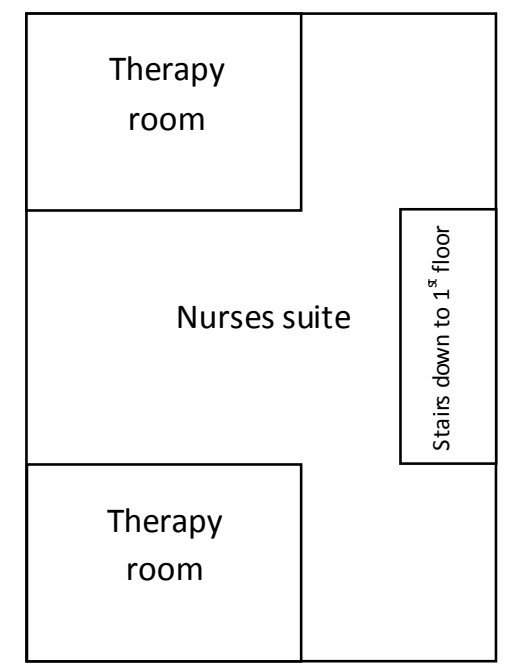

Set plan 7.6: Second Floor at Site 2

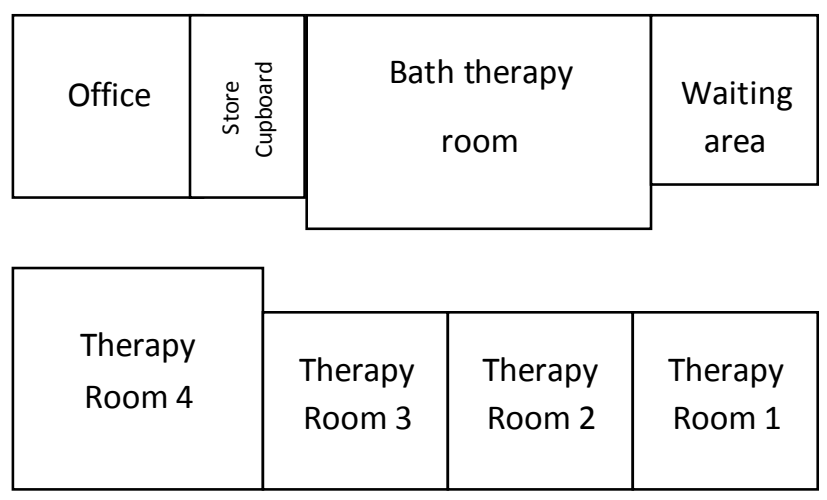

Set plan 7.7: Site 3 
On site again for four full weeks, here I conducted a series of general and therapy observations and (due to the size and nature of this site) also interviewed seventeen practitioners and nineteen patients. This produced interview data of 49 pages and over 26,000 words scripted into dialogue, alongside two fieldwork note books, that together reflect the whole and the essence of both these participants' and my own engagement. Including such volume would be impossible here, and also unnecessary as my aim is to expose, express and explore aspects of practice that participants find integral to the whole, yet are usually hidden or overlooked within mainstream research accounts.

Following the format of the previous Chapter 6, selections from this data are presented in scenes that capture both the essence of this performance (as I perceived it) and reflect the interconnections reported and felt between these people, their experiences and this setting.

\subsection{Act 2, Scene 1: Fresh flowers and sensitive souls}

Apparent from my first day on site here, were the extra measures that practitioners and staff go to in order to make the environment as welcoming and supportive as possible, for all those who enter. These included plenty of natural materials (i.e. wood), original artwork (contains the soul of the artist) and fresh cut flowers, to name but a few. These additions to the space may, by many, be considered non-specific affects, however I soon learned that for participants here, they were also communicants of the philosophy that underpins and guides these practices and that almost all considered them either integral indicators or even interventions.

I had plenty of willing volunteers waiting to participate in either interviews or treatment observations before I even arrived in the Country (in some contrast to UK where either potential participants were suggested to me or those I approached independently felt they often did not have the time to participate). They had all responded to my information sheets/invitations to participate that I forwarded to the clinic in advance, and like the practitioners here, were very eager to support my efforts, leaving their contact details with the assistants to pass to me.

One such volunteer was Celia. Meeting Celia was a very moving experience for me as her narrative reveals some harrowing details about her past that, perhaps for the first time, made me realise that I was in a different country from the UK and that these patients potentially have different histories and needs from those I may encounter in other places. During our interview, she spoke candidly to me about this past and I saw the effects it had had on her. 
Celia's narrative:

Anthroposophy was originally introduced to me, by a friend. I actively rejected allopathic care after becoming more educated, through reading etc. When we moved, not far from here, I already knew of this centre. I wrote and asked the (former retired) GP to take us on as patients. I receive the GP care, with mostly Anthroposophic and sometimes Homeopathic remedies, and occasionally regular medicines. I have previously attended for the bath therapy and eurythmy therapy and had a series of 'conversations' with [Anthroposophic Psychologist 1]. As a child, I spent a number of years in a concentration camp in Indonesia. Years later I began to relate my health issues to those past experiences. I have the lower level of insurance, and choose to pay the extra cost for the Anthroposophic medicines and therapies, when I need them. Cost would not prevent me from using this care.

I think this building [Site 1] dates back to the 1890's. It's a former private house and still feels like a private house. The front door is heavy and difficult to open, it is quite uninviting. The interior is much more inviting than the exterior. The light and the height make it much better. I like that it is 'simple' and not too luxurious. There is a wonderful atmosphere, and lovely light, which is better at the rear of the property. I'm glad I am a patient in that room, the front is rather dark in comparison. All the artwork here is 'in tune' with the philosophy, it lifts you and I like it, but I wouldn't have it in my own house. He [Anthroposophic GP 1] has a large pumpkin painting in his room that cheers me up. There are always fresh flowers too. It used to be shabby and very run down. [Anthroposophic GP 1] has made great improvements, with the interior decor. I value the effort they made to improve the environment, for example with the fresh flowers. Now it's more personal, it's nice. It also shows the practitioners value the environment effects, and that's a good attitude. It's particularly nice where people may feel unwell or worried etc. I had 3 years of group therapy, 1 day a week at a day therapy centre, for people who have been in concentration camps. That was in a hospital setting, in a woodland. They would bring flowers indoors. The setting affects patients, I always notice the flowers. Although, I cannot disconnect the therapy from the therapist either, it is an interconnected experience. A change of practitioner maybe even has a bigger impact than a change of space. I also believe a different therapist would alter the room, so it will always be a different experience. 
When I come here, I prefer to sit in the passage than the waiting room, the waiting room is too small. I find the room of [Anthroposophic Psychologist 1] too angular, to be comfortable. The desk and the chairs are 'very angular', and the only 'round' chair happens to be ugly - but at least more comfortable. They have this very tiny rest room, for the physiotherapy, it has an 'enveloping' effect. The activities inside the rooms detract from any road or external noise. Proximity to the train station is a bonus for some people, but this location is inconvenient if you travel by car, as there is no parking provision nearby. There is no bicycle parking either, and I come here by bike. This makes it not really an ideal venue for a clinic. It would be wonderful to move to a new setting, I know they have been talking about this for a while now. There is great comfort in the whole set up. The role of the Assistants is very important, how they answer the telephone, how they treat you is vital. I think relationships with Assistants can affect your whole perception of the clinic. They hear your general, practice concerns, and report these back.

Celia's account, for me, crystallised the essence of a 'sensitive soul', although you wouldn't necessarily know it from the outside. Her life, and the traumas within it, have all had their greatest affect on the deepest levels, from which she interprets her other illnesses or ailments to have permeated. So to, it may follow, the ability to heal must also affect these levels.

Like most of the patients here, Celia actively chose anthroposophic care over conventional medicine finding it a better fit with her way of looking at (and being in) the world. Achieved through education, this change was a conscious rather than reactionary move (she did not report any specific negative experience with regular medicine that pushed her this way) but is pleased she was able to 'get in'. Within this model, Celia finds her 'sensitive' needs are accommodated, even if the practical ones aren't. The heavy front door, the small waiting rooms and lack of either car or bicycle parking are all issues that can make it difficult to 'enter' the clinic (and ones she hopes will be resolved when/if the clinic moves to a new premises) but this does not detract from what happens once inside.

Beyond the entrance, all of Celia's attention is drawn to the qualities of 'feeling' within the space and her relationship with this. The GP she sees operates from the room on the south side of the building, which is much lighter and warmer than those on the north side (less sun) which seems important for her. Spending time in both sides, I found the different rooms had different 'moods', which easily translated into feelings: the south feeling 'lighter' and the north feeling 'heavier'. Like Celia, I did not like to spent too much time in the north room, as this 
seemed to make me quiet and a little depressed. For Celia's sensitivity then, this is an important observation that she finds vitally affects her experiences there.

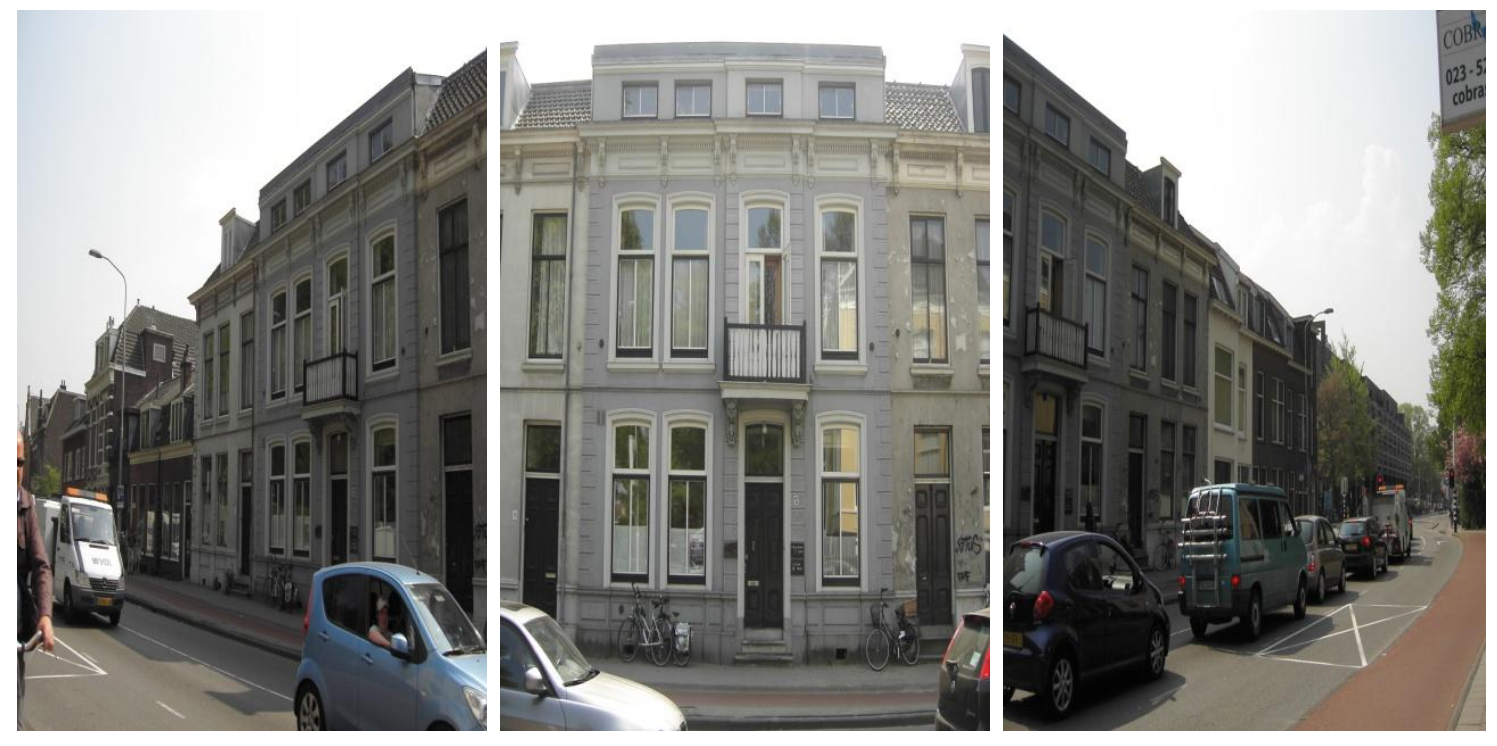

Triptych 7.1: The exterior façade of Site 1

Prior to entering therapy, Celia told me she also prefers to wait in the corridor, either on the stairs or the wooden pew/bench, as she finds the 'space' better there. Indeed, at times, the clinic was so busy that it could not accommodate all the people waiting and it became necessary to 'spill out' into the corridor. But for Celia, just two or three peo ple could make the space in the waiting room 'too small' to bear. Of more importance perhaps to some than others, Celia suggests that feeling unwell or worried can make such sensitivities more acute, and I likely agree with her. I personally did not have a problem in the 'waiting room', but then I was not feeling unwell or worried. Instead, I found this whole place like a 'welcoming old grandfather' (I don't quite know why, but this place seemed to have this distinct 'personality') as for me it may have looked a bit gruff on the outside, but it was stately enough to still be respected and full of wisdom and warmth. I felt this place and the people within it had much to teach me, which is a very different approach to those who may seek it as a haven (or fortress) of hope and healing.

Echoing a similar observation that was made by patients at the UK site, Celia has noted some clear improvements/changes in recent times, since her former GP retired (replaced by her current [Anthroposophic GP1]). The general improvements in decoration and presence of fresh flowers thro ughout she, therefore, associates with this character (aided by others) which suggested to me that this place is perceived by Celia as an extension of persona(s) and even an expression not only of what therapy goes on there, but how therapy is intended to go on. 

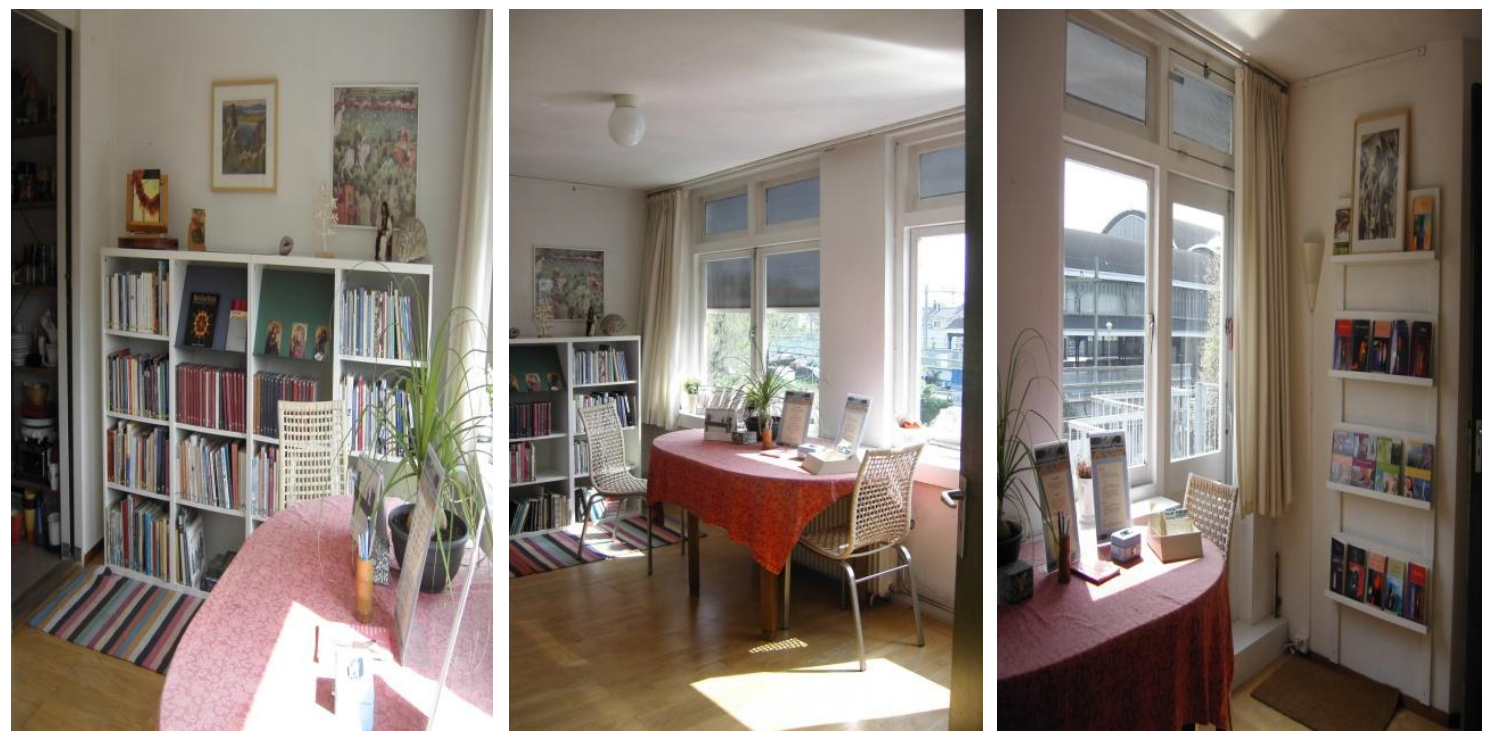

Triptych 7.2: Patients Library room at Site 1

Linking to the underpinning anthroposophical philosophy (that dictates therapy should attend not just the physical body but also the etheric body, astral body and ego/spirit) it is possible to see the importance attached to the presentation of space by Celia and others here (practitioners and patients), which also suggests how they should expect to engage with the space. Staged and performed by the current therapists, therapy here seems to achieve what it intends, in a positive way. It is curious that a boost in this coincided with a change in personnel (something seen also in UK when the former senior partner retired and a new one came in). Maybe this relates back to something Simon (in Chapter 6) said about accepting how things are and feeling powerless to change them. Certainly, I found that the majority of practitioners, upon taking residence in this Centre, had sought to freshen or make changes to their individual spaces, as their remit permitted. But perhaps for those who have been in situ for some time, the necessity for change fades, if they have already adapted? Moreover, in terms of the communal spaces here, those fall more under the leadership of the GPs than independent therapists, although my reports suggest they did seem quick to follow the lead set by this new character (Anthroposophical GP1).

This observation may reflect some of my findings also within CAM research and explain why I, as a 'new entrant to the field' have felt it necessary to make bold changes to my landscape (to better suit and support my own ways of thinking and being). The important thing to note here is the intention behind any changes is to create more/different/better 'space' for relationships to be made. Whether seen between Celia and these practitioners, or felt between myself and my research, altering a therapy space (or intellectual space) to 'suit your own style' may not be as superficial as participants in the UK thought, but have benefits beyond the practical. 

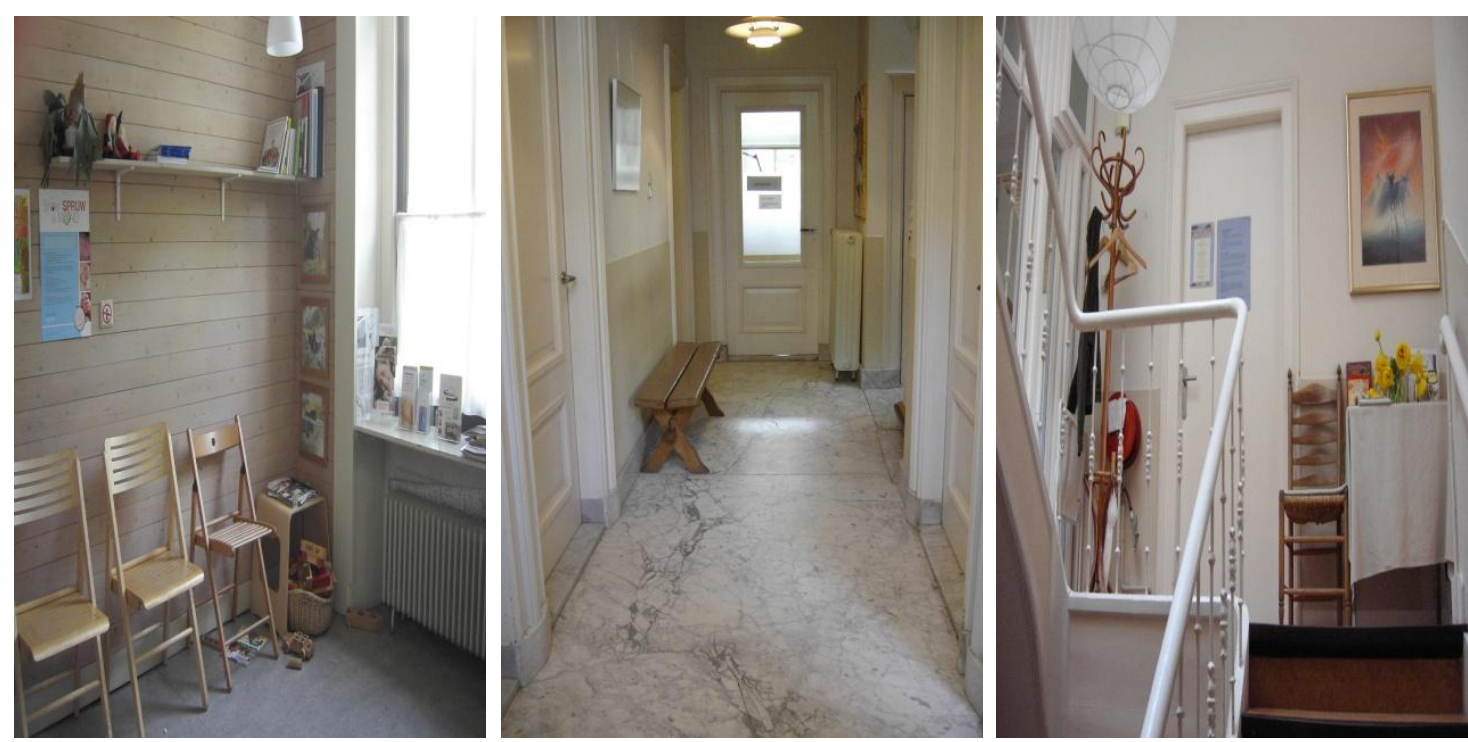

Triptych 7.3: Interior spaces at Site 1

Hearing Celia relate some of the 'touches' here (like the fresh flowers) to the therapy centre for people who have been in concentration camps, I couldn't help feeling her pain and the real/positive difference that 'little things' like this can make - to those sensitive eno ugh to appreciate them. Maybe then, the difference between these practitioners and the ones in the UK is that those (in the UK) may have never had to interact or deal with patients who have lived through such horrors as Celia ON THEIR DOORSTEP, and so do not recognise the full value of mediating their care accordingly. In truth, I had not fully recognised this either, until I met Celia and heard her story. It was most humbling that she chose to share this with me, and it was then I made the distinction about 'the doorstep'. I also lost family members in the last war (and previous), but this happened overseas and the immediacy of this was somehow 'removed' from my reality. However, after my conversation with Celia, I felt her immediacy and became more sensitive to these memories. I could not look at these very streets the same way again; it was like memories of German soldiers with their jack-boots marching through the town, dragging peo ple from their homes, were haunting me. Then, I felt the positive impact of fresh flowers, spiritual artwork and beautiful crystals, offering to carry me!

This raises a marked contrast between the UK and this Centre. There, all practical considerations were taken care of (even given priority) but aesthetic ones were not and generally seen as less important (Helen: "it's not that kind of place"). This raises the very clear distinction between a place that is good for treatments and a place that is good for people. In the UK, the former clearly won out (and patients generally agreed) and decisions made or things changed if they improved the practical delivery of therapy. Here, the opposite was true and the people mattered more, which was dramatised in the decisions made here, most 
notably the ways this place is staged with beautiful, original artwork, quality wooden and marble furnishings, fresh flowers in all the rooms etc. This seemed to also support the warm and friendly nature of relationships I observed between these people. Celia's observations, in this respect, appear borne out, that when a therapist alters a room this also alters relationships within. I also found everyone here lighter, less stressed and more relaxed than UK, which (while I was here) I was similarly 'infected' by.

They have a lovely expression here about 'carrying people until they can walk on their own'. I felt this setting, as both a 'space' in itself and a symbol of the care given by the practitioners to the patients, is an extension of that sentiment. Like Celia said, everything here "lifts you" and I found this too, in the tranquillity of the place and sensitivities of the people. However, this is more than soft towels and sweet music. Here I saw a genuine resonance between the spirits of the people and the materials within this space. I am not sure I can describe this adequately, but for me it did feel like 'being carried', 'embraced' even, by these surroundings, my spirit lifted and moved by the natural beauty of things within.
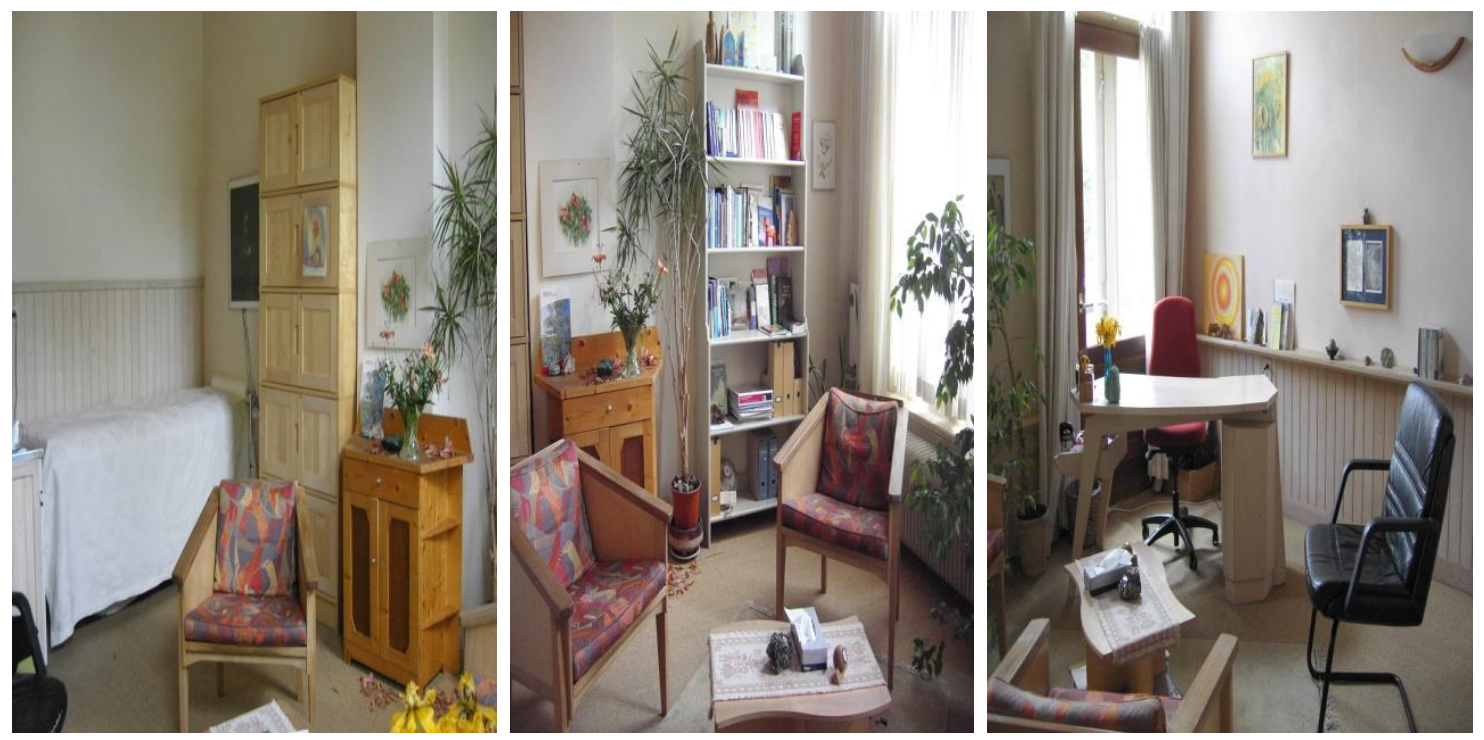

Triptych 7.4: First floor psychotherapy room at Site 1

I can only locate this within the vital quality of the materials I saw and the relationship between these, the anthroposophical philosophy that guides their practices, and the people. Natural materials and plants, crystals, and artworks or sculpture (each representing the different plant, mineral and spirit kingdoms) are the main differences I saw here, that were not encountered previously in the UK. In this 'space' people (as evidenced by Celia) felt to be cared for, carried and cured and that becoming better does not simply mean returning to the state they were in prior to illness, but where they arrived after recovery. Used for affect rather 
than any purely practical/economic benefit, this staging seemed to also convey different meanings; almost like how personal a choice is can influence how personal a choice feels. This was just one example of 'awakening' that I experienced here.

\subsection{Act 2, Scene 2: Therapy without 'borders'}

The driver of some of the most recent changes, Martin (not real name/Anthroposophic GP1), was both a central character in this production and also my key contact prior to coming on site. A very kind man and enigmatic character, I found Martin to be a popular addition to the centre, and someone I would describe as 'everyone's friend'. Due to the nature of his practice and my other commitments on site, I never got to observe him directly with patients (although I wouldn't have understood the Dutch anyway) but I did observe him numerous times in both a general and a social role and also learned much about the man, during our interview.

Martin's narrative:

I have been a GP for 10 years, after my medical training I did some GP training in Amsterdam and then spent my final year here [Site 1]. I had a very good relationship with my supervisor, and so I stayed here as an associate, also working 3 days in a nursing home, and then took over when he retired, around 7 years ago. I have 2,300 patient registered with me, and have to also run my business. I share the employ of the Assistants here with [Anthroposophical GP 2]. I always thought I would be an Anthroposophical Doctor. My parents were both anthroposophic fysiotherapists, working here, in fact my mum still is. They taught me that regular GP's were not good, they use too much antibiotics and such. In training, I always felt that you can be the Doctor you want to be, like it was not dependent on your training. But I felt like I was limited, by regular approaches to people and to treatments, so I eventually trained as an Anthroposophical Doctor, 6 years ago. Now I prescribe a lot of anthroposophic medicines, some homeopathic and some regular medicines. I also refer people for therapies; my favourites are the nursing care and the physiotherapy. A lot of people are 'without borders' and they need these to 'bring them back to themselves', to see the boundary where they end and the world starts. People need to feel their borders to be well again. I sometimes refer to art therapy and the psychotherapy. I may also suggest acupuncture and chiropractic. Therapies and medications are not always insured, which can make the work more difficult, I cannot always prescribe what I want if the patient can't pay for it. Also, if a remedy is forbidden in Holland, I have to import it from Germany, which has delays. If the patients are sufficiently motivated, 
sometimes I will pay for it. My aim is to give the patients support in regaining their own health. I help them to be able, but they have the responsibility.

This house [Site 1] has been a therapeuticum for 40 years, originally starting as a GP's home with the family living above then, as the family moved out, more therapists moved in! We rent it from a trust now, a foundation, and we each pay either rent or a percentage commission to the foundation. I wanted to work in [this City]. My family were all settled here and my children were in school, so when the opportunity came, I took it with both hands. We have all the advantages here of a town, and the character of a village. I like coming to work here, everyday, it is a great place and a great team. The train and bus access is good, the parking is more of a concern. I really like the age and style of the building, but I would like a bigger one, this is not ideal enough. I would love a more quiet place. I think place and self are integral parts. I want a new building, with more space so I can train GP's here and have a separate kitchen from the Assistants clinical space. Our waiting area is also too small and crowded, this can make patients feel anxious. Of course, a large one could make people feel lost. I would like all of the Doctors and therapists to work together in one building. I think that seeing each other often will enable us to provide better therapy and enable better dialogue between the team. I have been trying to unite the two buildings for 5 years now. I am a big motor of the team, I do a lot or organising. It is hard work.

My current space is good for myself, and good for the patients, so they tell me. I think that anything beyond physical care needs to be in a good place. Everything is important. These stones are symbols of remedies, my 'museum' were mostly gifts, and I bought these paintings from patients of the art therapist. I like to have my boys here too [children's portraits] and the flowers, they make people feel welcome. Of most importance here, is the stairs. This is the one place where we can all meet and view each other. I would like to have more interaction with the nurses and other practitioners. I feel my environment is important, even before I knew it. I am happy in my own space, I love the light and I have enough room. I'm glad I don't work in [Anthroposophic GP 2's] room; that one is on the north side. In the nursing home, it was a very communal, family environment. The residents and staff were together, being together, cooking together. We treated people like it was their place, and we were only facilitators. We even would ring the doorbell, though it was always locked, for safety. 
The work we provide here is good, but it could be better. It is a problem that I cannot easily refer people to physiotherapy or psychotherapy if they cannot make the stairs. We really need an elevator. I would love a garden, at the centre. Sometimes, I will sit and discuss patients in the garden, or walk with a patient along the river across the street. They often talk of feeling 'carried by the team' who work here. I think that having a social worker in the practice [Anthroposophic Nurse 1] makes a big difference to the scope of care provided here. I am also influenced by external pressures and barriers. I bring myself into work, every day and use my insights, but do not preach 'conversion'. We have a lot of Turkish migrants here and they have cultural and generational barriers that influence how I care for them, and the role of these in their dis-ease. They bring me gifts, if I like the gift I will leave it here, if I don't, I will take it home, I have a lot of 'evil eyes' at home.

Martin's whole approach to medicine here was one that has developed as he has developed, growing in philosophy as he has grown in philosophy. I have described this as 'therapy without borders' because only through adopting an anthroposophical approach has Martin felt free enough to be the kind of Doctor he always wanted to be, breaking the borders set by conventional medicine. Even coming from a family that followed anthroposophical teaching (at home and work), mainstream discourse managed to limit him. Since then, he has been able to become less reliant on conventional drugs (for treating problems) and instead refer patients for therapies that will help them "to be well again" and "feel their borders".

A direct response to his training, this method of working is (by its very nature) different from other General Practitioners (although not here), meaning these patients get a different experience and outcome than they would in a regular clinic. Employing insights and education, Martin reports connecting well with his patients, and also sees where he could do more for them, if his environment supported him better (it is quite adequate, but he wants more). This is why he has made the improvements that Celia spoke about and why he is so keen for a new 'house' where all the practitioners, across the different sites here, can work together and support each other for equal practitioner and patient benefit.

In many ways I found Martin a similar character to Simon. The main difference between these was that Martin already knows (from his upbringing, experiences and education in anthroposophical medicine) the positive impact that the environment for therapy can hold, and so implements this, whereas Simon feels there may be some benefit but needs to see 'proof' so he can justify the investment. Indeed, it was quite refreshing to be in the company 
of someone who felt able to justify his approach to practice based on everyday case evidence rather than be beholden to the latest clinical trials or evidence. Sometimes, it seems, the best evidence can be right there before you.

I found all of Martin's performances to be delivered with a natural and easy authenticity, supported by this close and communal network (of other practitioners and family). Having been the catalyst for changes that all here have embraced and enjoy, I found his lead to often be followed by the others, for example when he first expressed enthusiasm for my study and shared this across the therapeuticum, and then in the nature of the welcome I enjoyed when I arrived on site. Indeed, my first memory of meeting Martin was how he met me (and my family) at Amsterdam Schipol airport, with a very large bunch of yellow tulips (the national flower of Holland) before taking us to our rented house. This kindness and generosity was followed up the next day, when Martin had arranged for one of the part-time GPs to collect me from my house and take me on an introduction tour of all three sites, so I could begin to plan and run my study, efficiently.
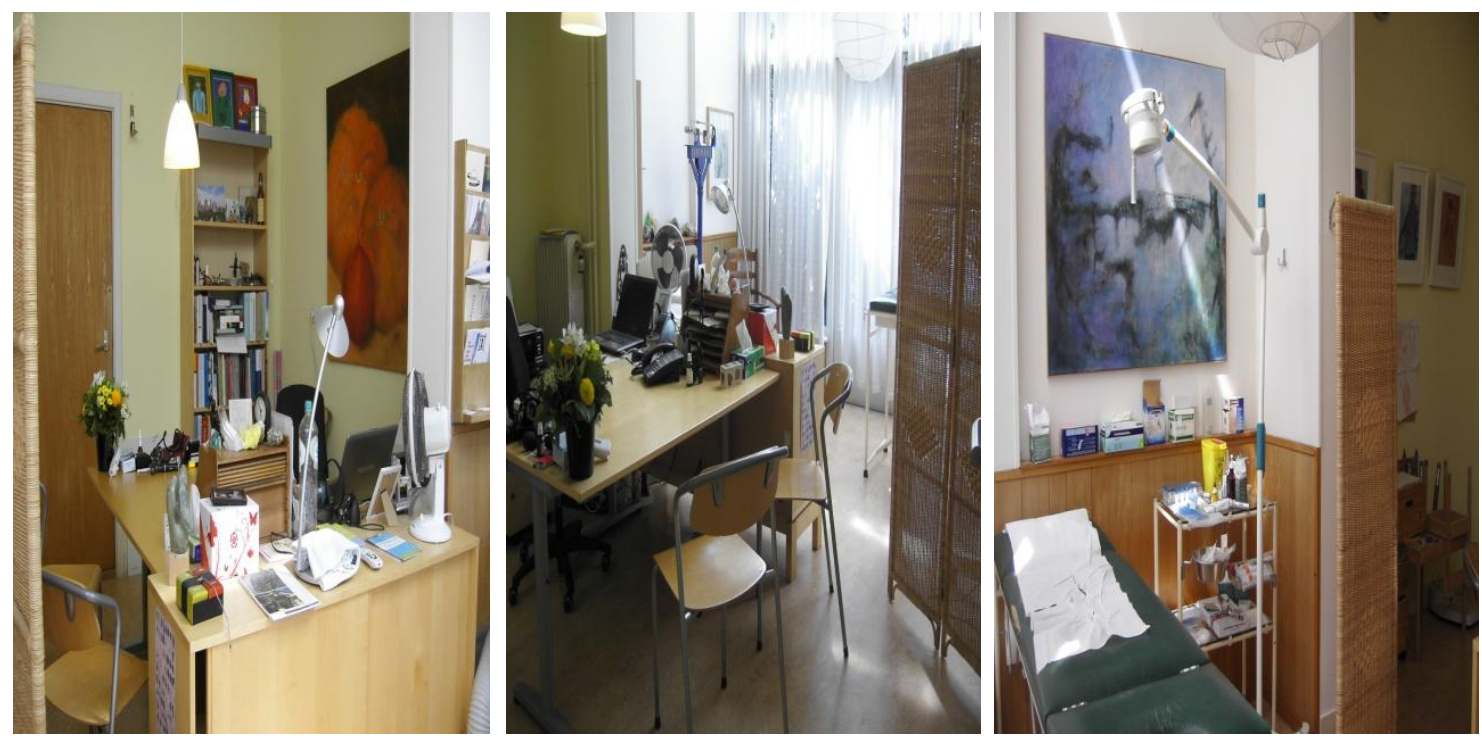

Triptych 7.5: General Practitioner 1's room at Site 1

In terms of the quality of data I gathered here, touches like this (similar to the fresh flowers noted by Celia throughout) really made a difference. I felt embraced and cared for, from the start, which made it all the easier to make connections and relationships (even friendships). Feeling more accepted than I had in the UK (something I found a curious position, given that I held a shared background with practitioners there), I was immediately more relaxed in my data gathering, more like 'an insider' watching, perhaps even noting more depth in the data as a result; the participants (also seeing me as an insider) appearing less guarded than they had 
been in the UK. I also found I had freer access to both spaces and participants here, as part of the same scenario of support rather than control. I felt this fostered a mutual respect between all parties, the idea that everyone feels like an equal and treats everyone else in the same approach. This certainly made my experience here more pleasant and less stressful than it could otherwise have been (as a novice researcher in a 'foreign land') and I feel this quality influenced both the data that I did gather here and my relationships with this. If this ethos had even half the impact on patients that it did on me, then this is an observation worthy of noting.

This easy, relaxed style was something that permeated every part of Martin's practice, underpinning the happy, family relationships that seem to exist here across the whole staff. From his perspective this can only get better if, one day, the whole therapeuticum were able to work 'under the same roof' where all can support each other and 'know their borders'. Until then, he knows his approach will 'not be ideal' (like Simon's in UK) and this does cause some frustration. However, I never saw this interfere with his practice, his natural enthusiasm and conviction seeming to, again easily, override all.

This does not mean his work is without conflict, entirely. Previously he has faced some inner conflict, when he tried to be the kind of Doctor he wanted by desire alone (without the support of additional training) and found the restrictions of mainstream practice challenging to his personal discourse (originating from his childhood). This led to an unsatisfactory situation that he pragmatically remedied, taking further training in anthroposophy and changing his treatments and approach so no such conflict remained. Now he blends these two opposites in a way that satisfies both his discourse and his patients. I found this 'can-do' attitude was characteristic of the man and also means that when there is an issue to be resolved, Martin can always be relied upon, to find a way (for example when there is difficultly sourcing a specific remedy or medicament). This commitment to patients always getting the best possible outcomes was manifest in additional comfort, alongside the treatments and therapies he provides/prescribes, which Celia previously noted ("there is a great comfort in the whole set $\left.u p^{\prime \prime}\right)$. Within the framework of this discourse and therapeutic approach, it seems Martin always endeavours to make sure everything that can be done to aid healing and care is done.

\subsection{Act 2, Scene 3: Good vibrations}

A clear example of this is the 'creative care' I observed being delivered to Nora (not real name) under the direction of [Anthroposophic GP3] and by one of the nurses (Jenny, not real name). I had met Nora during my third week on site. She had volunteered to participate via Jenny the previous week and so we made arrangements for me to observe and interview her the next 
time she attended. Nora is an unusual patient in that she is currently receiving a regimen of 'mistletoe infusions' as cancer therapy. This sort of thing is rare here, with only two of these patients in four years, which only this GP prescribes and only Jenny supervises.

I was already in the nurses suite (second floor at Site 2) chatting and watching Jenny prepare for Nora's session when Nora arrived. Everything was already set up in the therapy room at the front of the building; the treatment bed had extra pillows that Nora liked and was also dressed with the same towels as Nora usually has. Jenny explained to me, as she hung Nora's infusion bag onto the net curtain (the wire, near the head end of the bed), that when she worked in hospitals it would be unthinkable not to have the correct equipment, like a wheeled frame for this, but here they just make do and it works fine.

Just then, Nora arrived. She looked a little tired after climbing two flights of stairs, but pleased to be up in this space now. She paused briefly by the 'kitchen table', leaning to rest on one of the chair backs. The sun streamed in and birdsong could be heard in the trees, just outside the window of the small therapy room, as Nora entered and climbed fully clothed up onto the bed. She lay down while Jenny covered her with a thick yellow blanket and waited for [Anthroposophic GP3] to arrive. Jenny, dressed in crop trousers and a floral blouse (not at all 'nurse like') then brought Nora a cup of herbal tea, which she sat on the chair to the side of the bed and they exchanged some social chatter (in Dutch, which I didn't unders tand).

It took around 30 minutes, after Nora's arrival, for [Anthroposophic GP3] to come in and begin to administer the infusion. By now, Jenny had also prepared a hot water bottle for Nora's feet, to be positioned under the blanket after [Anthroposophic GP3] departed. I watched quietly as [Anthroposophic GP3] entered the therapy room, greeted us all, and then spoke softly to Nora, checking how she was feeling today. There was a calmness to this whole exchange unlike any infusion I have ever witnessed in a hospital environment, made all the warmer by this soft environment of 'cloudy' peach walls, comfortable linens, sunlight and views of the trees outside (plus the absence of lots of 'medical equipment'). [Anthroposophic GP3] was casually dressed in corduroy trousers and a checked shirt. He took Nora's arm gently, together with Jenny, and found a good vein to introduce the needle (cannula). He sterilised this with a swab, quickly connected the infusion which jenny secured with tape, and left the room bidding Nora 'good day' (taking any waste with him). Jenny then wrapped the connected arm in a yellow towel and left Nora to rest - checking back on her every 15 minutes to monitor both the progress of the infusion and Nora's general comfort. This was usual practice, but perhaps 
more important today as both [Anthroposophic GP3] and Jenny, while happy to support Nora's decision to participate in my study, were also keen to ensure this did not tire her too much.

Jenny had informed me it takes between 60 and 90 minutes for the infusion to complete, during which time Nora will normally read, drink tea or sleep. Today, however, it had been agreed that Nora and I could spend up to 30 minutes completing her interview, after which I must leave her to rest. We did stick to this, however there was a slight period of social talk at the end (which was unrecorded), during which Nora did seem to grow quite tired and took us technically 'over time'. While completed at Nora's instigation, Jenny felt she had had enough by now and that it was important that she rest, so I wished her well with her therapy and left the room.

Reflecting on my time with Nora and the data she shared with me, I felt very humbled and privileged that this courageous lady was willing and able to contribute to my study. She has been through a lot up to now, and therefore has a lot to teach those who wish to listen.

Nora's narrative:

I moved to [this City] after leaving my parents home. I took a recommendation from a neighbour for a local GP, and for a number of years never knew that he was anthroposophic. I was well, and didn't need to come. When I became ill I was happy to discover that he was also anthroposophic and homeopathic. I have been very ill for around 20 years now. He [Anthroposophic GP 3] keeps me going. I get my GP care from him, always with the anthroposophic medicines. The student GPs prescribe only regular medicine, and he [Anthroposophic GP 3] sometimes bans this! I have previously had individual and group eurythmy, art therapy and psychotherapy at [Site 1] and currently I am getting 'mistletoe infusions', as cancer therapy. My insurance and how useful this is depends on who is in government, particularly with regards to mistletoe therapy. So far, cost has not prevented me from having the therapy and I have a financial 'buffer', some savings, to help.

This Centre [Site 2] is an old house, big, high, old fashioned and typically anthroposophic. It is much better than a new one. Whenever I enter [Anthroposophic GP 3's] room, I always walk to the window to see the Cathedral. If ever [Anthroposophic GP 3] has to take a phone call or something, I drift back to it - it is a great view and I never get bored. At [Site 1] they have some nice paintings, especially on the landing area. I notice those more than here. Regular hospital is 'heavier'; now I 
know rooms can have influence I am more aware of space, but 20 years ago I didn't know this, so I paid less attention. The resonance of objects may be dependent on awareness of their potential. My previous GP was in a modern house, and I didn't visit much. When the cancer returned, 6 years ago, I received vibrational therapy and was told that my house was a bad influence on my health. Geopathic stress interventions attempt to transfer bad vibrations into good vibrations. I am sensitive to these and conscious of geopathic influence now. Therapy is always located upstairs, high houses and steep stairs are a problem when you are ill. This space doesn't really suit the infusion therapy, it is more for the 'rubbings', but it is good place with good vibrations. I can smell the oils when I enter upstairs. There is a lack of clinical kit, but that isn't important, they just tie my drip to the curtain track. The interior decor reflects the anthroposophic philosophy, round and fluffy, textured and cloudy colours. I like it, it is good, but the room at the front [part of Nurses therapy suite] could use more light.

I am glad to have this therapy here, near my home, where I don't have to drive and there aren't so many sick people. I have been in hospital often and also had the infusion therapy elsewhere, more clinical. The front door is heavy, which is a problem if you're not feeling strong. I've always found it odd that the people visiting are likely in pain or ill and yet there is a heavy door to push! The door is difficult, and this creates worry. This centre feels welcoming, more caring, because of the people. You 'see' the Doctor, and not so much the surroundings. Practitioners are very important to the experience of therapy. Familiarity and history makes it better. Last winter, when I had pneumonia, \{Anthroposophic GP 3] did not allow antibiotic use but instead called and visited me every day, to take care of me. It was very heavy snow, but still he came. That felt very good.

Like Celia, I found Nora a very sensitive individual; sensitive both to her inner self, how she connects to other people and the impact of her surrounding environment on her health and wellbeing. I found it interesting that, when she was well, she did not consider it essential that her Doctor was anthroposophic, but on becoming ill found this an advantage.

It seemed clear to me that Nora considers the approach taken to tackle the problems her illness brings are met better in this environment, with this approach, than they would be under regular medical care. She makes this judgement from the position of having experienced both, using each as a means to critique the other, against her developing knowledge of how the people or things around her can impact her (either positively or negatively). 
A product of her unique illness journey, she uses this now to make observations that would otherwise be overlooked, such as the quality in spaces ("hospital is "heavier") or the quality in relationships ("You 'see' the Doctor") and relates these to both her satisfaction and outcomes. This was something I found many others here also capable of, although perhaps in less detail than Nora, just more aware of the 'feelings' they have rather than the potential theories that underpin these.

Her position, is this respect, is not so different from mine and which I also bring into my 'meaning making', throughout this study. Relating back to an observation I made in the earlier literature review (Chapter 2), Nora here exemplifies the unique role of personal experience in appraising and engaging with different therapeutic approaches. In her case, she has become consciously aware of the value or impact of the people and things around her after experiencing 'vibrational therapy', and uses this understanding to distinguish between good influences and bad influences in a more informed way. Supported by this 'understanding' she engages every new event or experience, always referring back to this. For anyone who has not become aware of such issues (either by education or experience) it would be much more difficult, even impossible, to conceive of the nuances that Nora details, never mind be able to recognise them in practice, assign value to them and articulate this to others.
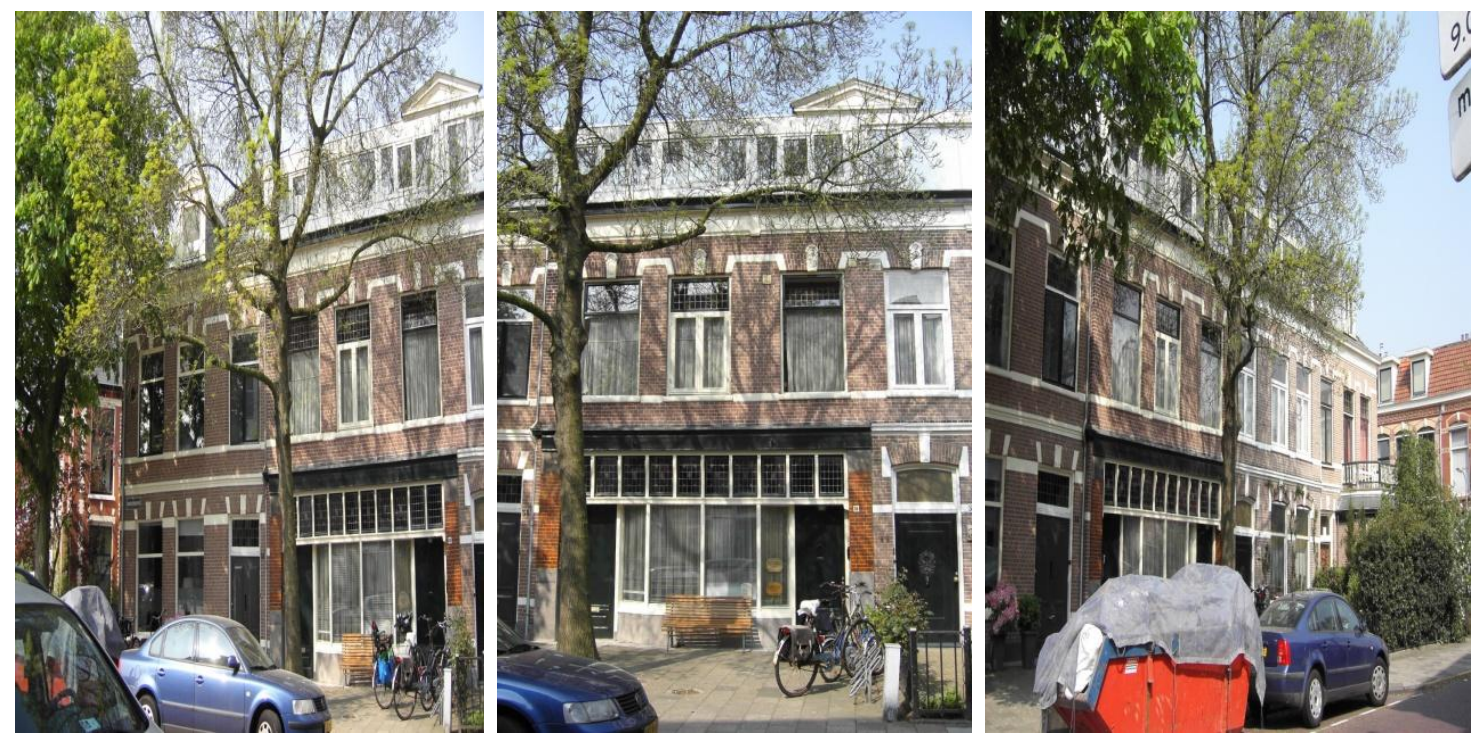

Triptych 7.6: The exterior façade of Site 2

This may explain (or even excuse) the omissions made in this regard by other CAM researchers. First, there is a common discourse in healthcare (and CAM) research that favours physical approaches and interpretations of treatment, which some practitioners will seek to provide, as seen in UK site, and researchers will report on. Then, there is the personal experience (or 
alternately a lack of this?) of individual researchers with different healing approaches, as I demonstrate here, determining how each will engage and interpret any phenomena they observe. Either of these two states can create or limit the 'space' for potential relationships within research and reported results in practice. Nora, it seems, has created the potential to identify, engage and resonate with subtle and sensitive energy effects, both within her therapies, with her practitioners, and in these settings. I think any researcher can learn much from this.

Certainly, Nora's appraisal of the care and everything on offer here (not just the remedies or therapies) is that it is both anthroposophic and salutogenic. Here, as at Site 1, there are also elements of the three kingdoms (plant, mineral and spirit) within each of the spaces, that reflect and actively communicate these intentions and affects. Part of her satisfaction, then, is that this whole staging and production communicates understanding of her meaning-making processes as it seeks to support healing (whatever that may ultimately mean). This gives her comfort and confidence in the best possible outcomes. At a base level this may not be so dissimilar to Helen's experience (in Chapter 6 ) who found provision there was a good match and support to her understanding, albeit in a different discourse to the one subscribed to by Nora. For Nora, matching expectations (however different these may be) could again be integral to understanding her patient experiences. Recognising Nora's personal bias, which everyone has, is as important to this data as recognising my own (something I have endeavoured to do throughout this thesis) as without this the unique perspectives and insights of participants can neither be embraced or critiqued.

A further striking aspect I want to explore, within Nora's experience, is the value of the personal service that [Anthroposophic GP3] provides for her (even 'out-of-hours') and the impact this has on her confidence in their relationship and his care. Initially I found this a pleasant harp back to the kinds of service most GPs provided during the last century. More recently, I have learned just how critical having this level of support can be, both in terms of compliance with a regimen of CAM care and in general satisfaction and clinical outcomes.

Back in 2011, my then Homoeopath (for the last 9 years) emigrated from UK to Cyprus. At the time, we were all in good health and so, rather than seek a new homoeopath, we decided to try to maintain our relationship via Skype, with regular appointments every couple of months as we had in the UK, to ensure continuity of care. Whilst I cannot say the experience was anywhere as good as having a face-to-face relationship, this felt adequate. Then, early in 2012, my eldest child contracted a viral cough which left her with a wheeze (not quite a diagnosis of 
asthma) and I was unable to raise my Homoeopath due to combinations of time difference and an unpredictable Cyprus internet service. As she visibly declined, I was left with no option but to seek conventional care and she ended up on an inhaler, which helped. Some days later, when I did get to speak with the Homoeopath, a remedy was prescribed and she was able to reduce and remove dependence on the inhaler. A little over a month later, my youngest child developed the same problem. Recognising this, I sought to contact our Homoeopath, but still ended up visiting the conventional GP, who delivered the same diagnosis and prescription. Again, when I did manage to speak to the Homoeo path, we were able to restore her condition, but this kept recurring, each time she contracted a viral cough.

Then in March 2014, my youngest child had an episode which did not respond to the delayed prescriptions of the Homoeopath (did I mention that it would often take 10 days for a remedy to arrive, if posted from Cyprus?) and the condition became so serious we were admitted to hospital by ambulance, with oxygen, where we stayed for 5 days absorbing every medication and $x$-ray they could think to blast this tiny system with. At this point, the Homoeopath felt too removed from the situation to prescribe adequately, especially given the suppressive nature of the hospital medications, and advised to 'pick up the pieces' when things settled down and were stable again. Unfortunately, they did not become stable again, having 5 more similar admissions between April and December 2014, each time pumped with more $\mathrm{x}$-rays and drug regimes.

The feelings of abandonment by the only system of medicine I admired (okay, by the individual practitioner, not the entire system) were very hard to bear; more so because the situation felt 'forced' by the dominance of biomedicine and all (the suppression) that comes with this. Knowing the ambulance service and hospitals are always there to deal with a crisis was, for me at least, no comfort compared to the proactive management by a consistent individual. Moreover, I felt devalued and disempowered by the discourse and system that is current UK healthcare. No one was 'taking care' of us and this caused me much anguish, damaging my own mental and emotional health. Eventually, I could take no more, and following a discussion with the Homoeopath sought a new Homoeopath, to take over caring for my children in December 2014. This character, more local to me, able to 'see' the case fully and therefore happy to prescribe, and available whenever I need him has given us all the courage to again move forward in supported health (not 'controlled' health). There have been no further hospital admissions and with a protocol for decline and recovery that does not mean relying on heavy and suppressive medications, we are all (currently) happier and healthier. 
Nora's experience of 'close care' may have similarly impacted her too. She spoke very warmly of how this kind of care supports her health, whether in the regimen I observed her receive or the daily home visits she enjoyed last winter. While it is not for me to say what direct impact this has all had on Nora's pathology, I do know that she values it highly and that she feels it does make a significant difference to her. Based on this, there may be a case for understanding the complex process of pathological change by appraising the whole of an interventional approach (including the influence of prevailing discourse) and not just the parts.
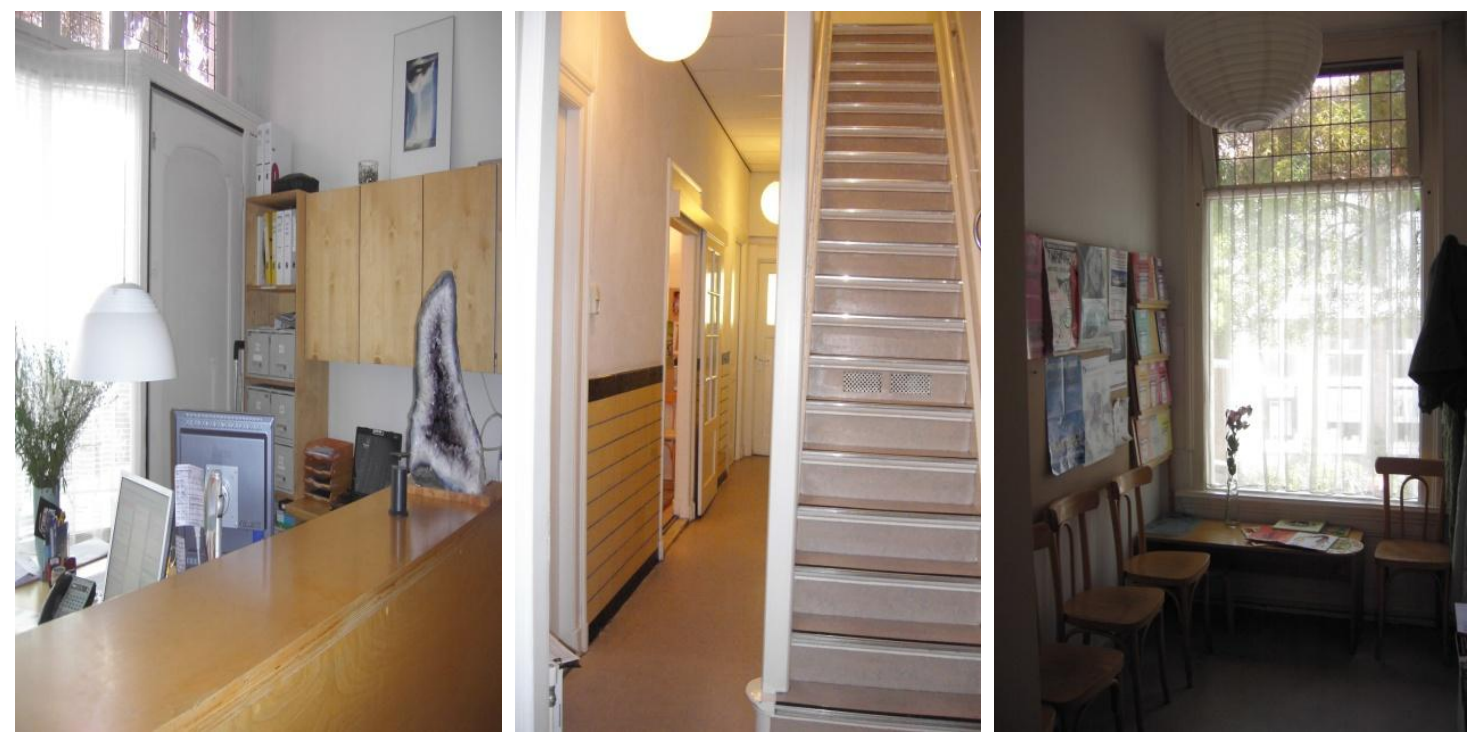

Triptych 7.7: Interior spaces at Site 2

I felt Nora gained much from our time together. It seemed important to her to share her experiences of therapy here (she insisted on completing our interview even though, for the latter part, she was clearly tiring). I sensed it also gave her some 'accomplishment' that perhaps she had grown unused to. I was glad that I could enable her in this.

More than this, however, Nora's account also represents a direct challenge to local discourse; evidenced in her comment about how useful her 'insurance' is being dependent upon who is in government. This suggests that the healthcare options of citizens are not always free but rely on the influence of politicians to support them - sometimes this is includes wider, alternative choices and sometimes it does not. This observation has been previously noted by Martin when he talked about the difficulty of prescribing some remedies, and was also raised by others here. 


\subsection{Act 2, Scene 4: Medicine in the right measure}

The man who perhaps most epitomised the measure and vitality of the anthroposophical approached delivered here, for me, was Emil (not real name/Anthroposophic GP3). Emil is a slight and slender man, kind in the eyes and thoughtful in his manner. A GP in this practice for many years, I found him a comfortable and settled character without the same desires for change that Martin is so keen to pursue, being rather philosophical about the future instead.

As with the other GPs, I never got to observe Emil directly with patients either, but did again spend some time observing him in informal situations with the other staff that I used to help explore aspects of his interview dialogue.

Emil's narrative:

I knew at the age of 5-10 years that I wanted to be a Doctor. We lived in The Hague but did not like to be there and my mother, who had been raised in [this City] and was an educated, truth seeking woman, became ill with cancer. She would not use regular medicine, instead she trusted her care to an Anthroposophical Doctor here [this City]. He said to her "you will not die of cancer, you will die when it is your time". Through anthroposophy, her thoughts and her heart came to agree, her depression healed and her cancer was cured - she had surgery but no radiotherapy. I was 6 years old when anthroposophy came into my life fully; my diet changed and I began to say prayers. At 8 years I attended a Steiner/Waldorf school, which I think was good for me as I was only intelligent in the head, not with feelings. I became a more integrated self. When I came to study medicine, at University, I found the regular view too limited so I joined an anthroposophical student group. This was inspiring, but I was unsure if I wanted to be 'an anthroposophical man'! I witnessed old anthroposophists to be 'more vital' than old professors.

I did my GP training in anthroposophical practices. I spent time at [Site 1], also an anthroposophical psychiatric hospital in Germany and later the practice of Ita Wegman. When I had completed my training, I returned to [this City] and joined the practice of an older Doctor, intending to take it over, but he wouldn't let go of it - I think he 'needed' his work - so I ended up building my own practice from scratch, which is how there are two anthroposophical GP practices here [Site 1 and Site 2] now. I generally see patients on the first floor, and will use [Anthroposophic GP 4's] room on Wednesday afternoons, if a patient is coming who cannot use the stairs. I aim to 
vitalise people, and make them free, so we 'carry' the patients, until 'they can walk'. I like to have that 'Raphael' quality. I prescribe mostly anthroposophical remedies due to the conviction these are helping, and while getting the right medicaments is more difficult than it used to be, I do not like to prescribe suppressive medicines. These are not as aggressive as regular medicines, forcing a response in 'side effects'. I think anthroposophical remedies are in the middle between regular and homeopathic medicine. They are the right measure to bring people into 'the right place'. Taking complaints away is not the same as development, and people are usually ill due to stagnation and no development so my first question is always to discover "where is the element of development?" I try to integrate care approaches and I will prescribe anything I think may be therapeutic, in the sense that it contributes to the development of the patient. I look to identify the lifestyle or social causes of illness and have prescribed singing, for a patient with asthma, also sculpturing and diet therapy. Every action which is real therapeutic, contributes to a development of patients. I very much like the art therapy and eurythmy. These open the soul, bring people into a safe place, and then into movement and development. Every time you express you own creative force, you are developing. When remedies are unavailable, I feel like a friend is in exile. I find agnostic and materialistic people can be depressive in their soul.

I used to work in my own home, and [one of the Assistants] worked for me. I moved my practice into this house, and joined with an older colleague, when my own home became too full of children! This house [Site 2] has good proportions, good light and a good 'spirit'. When we moved in, I had mostly the younger patients, so I took the first floor room and we were able to influence the design, and we kept many original features for example, the high ceilings and the room layouts. Our coffee room is 'just' big enough. I like to be elevated, I like to see the sky, the trees and the Cathedral. Everything is intentional. The flowers, wood and art are all personal choices, they create an atmosphere where I feel well, and can breathe out as well. I use these cards with the patients; this one has Jesus with Martha and Mary, you know the story? I give this to ladies with the menopause, to reflect on. I also have this one here, it is Jacob and the angel - it reminds me to 'carry' patients. The furniture was made for me, by a patient. This sculpture was also made by a patient, who I sent for creative therapies. Choosing things that are beautiful creates movement in emotions and this affects breathing, healing. I hope the space has therapeutic effects. People say 'this place' feels good, they feel well here. This space carries me also. Therapeutic events are 
whole, the space, the person and the treatment. I like to work in this house. A good space brings the soul into movement.

I do not like offices or centres that are purely functional; this makes it harder to discover the essential things. If there is nothing in the space that says you are welcome' then people do not feel welcome. It is important for people to open up themselves to make sense of their problems or issues. Natural science definition of what is a medicament is a substance with certain therapeutic effects and always side effects. If there are no side effects, Doctors say well then it is not a medicament. I believe a real medicament is always in the middle. I am afraid that homeopathic high potencies also force, and do not 'let free', but from the other side. I am an old fashioned Doctor and I feel, to be a good Doctor, you must be the medicament. Relationships are central. I give instruments so they can work on themselves. I will also give urgent cases my private number, so they don't get conflicting treatment outside clinic hours. I bring myself into my work, and my work into myself. I am a better practitioner when I am in my own space, with my own things. Being a 'guest' in a centre is very impersonal. I instinctively felt [this City] was a good place to work, and to be. It is a very special place both due to its' geographical position, between North and South Holland, and its unique history. It has endured much and its survival brings a very special quality. I think the atmosphere in this region holds those memories. We are beautifully situated here. I think the measure is right. I am content here. There are plans, across the therapeuticum, to move to a new house. There are good reasons for this but it may be more about thought than feelings, a rational move. I think this will be alright for the future, but it must 'be right' to happen.

Emil's narrative reveals the nature of his character and how this is integral to his work here. Like Martin, Emil was also raised within an anthroposophical household and education, which provided the foundation for him to be aware of and enter into this kind of work. Emil also found, while completing his medical training, the scientific approach to be too restrictive and took support from an anthroposophical student group (at University) to remain connected to his original discourse. Since then, his path has been consistent in joining and then developing an anthroposo phical medical practice.

I found Emil to be a fascinating character, calming and knowledgeable in equal measure, who seemed able to 'see' different dimensions of being and act accordingly (always in exactly the right response). His experience, understanding and expertise are unique to him and this felt to 
be the case when listening to the reports of his patients also (many of whom contributed to the study but cannot be featured individually). Set against some of the other characters in the therapeuticum, and even this site, he presented the closest I found to 'an anthroposophical man', vital in his eyes and his approach to people and seasoned in how to manage conflicts with mainstream medicine.

His whole performance said a lot about how the man and his role really are integrated. He talks about his approach to this care as if it arises from himself, not from any external training. He desires to fulfil the qualities of 'Raphael' in as much a personal aim as a professional one and uses himself to look for the links, finding the opportunities for development and 'carry' his patients until they can walk. He also has his own ideas, about medicaments and remedies and executes his use of these according to his own philosophy, prescribing anything he feels may contribute to the wellness of patients. His prescriptions are therefore personal and unique to him, supported by anthroposophy, as they appear to others here also. As in anthroposophy, everything has a reason and I saw this shining through him.

Emil's current 'space' is very important for this, as he stages it with everything around him that will help him to be the best Doctor he can be. For example, the religious postcards that he will often give out instead of prescribing remedies or even the presence of beautiful wood carvings, sculpture and flowers to 'move people's spirits'. A unique contribution, that no other practitioner in the whole therapeuticum can offer, is an unparalleled view of the stunning nearby Cathedral directly from his large French windows and balcony. Several patients commented spontaneously that they find this uplifting, before they even begin consultations. Emil too, finds this a supportive aspect to his work (although positions himself in the room where he has to choose to look at it, rather than be do minated by it) and notes how he finds working in any other space less satisfying. This is also partly because he does not always have to hand the things he needs, when in a nother space (for example the religious postcards which none of the others seem to employ). In such instances, not only does the material content of his care change but so does the emotional place from which he delivers this. In this space, he is centred and grounded, content and complete.

More than this, Emil also looked very at home in this place too, whether in his consultation suite or together with the others in the cosy little kitchen on the ground floor, sharing coffee and cheese/crackers. This is not just somewhere to work, it is somewhere to 'be', together with others who also understand the value of 'being'. I learned this cannot be separated from the acts that Emil and others here perform for their patients, but integrated into their honest 
and authentic ways of connecting all things (people, plots and place) for right development. For Emil, any appraisal or evaluation of his care, delivered in another place, would be a different performance.

Emil was not the only person I met across the therapeuticum (combined clinic) who felt this way, and is also the reason he feels unsure about the realities of relocating his practice into a new house, knowing he will never again enjoy the aspects (such as his view of the Cathedral) that he enjoys here. He acknowledges there are benefits to anticipate, for example that it would be better if patients did not have to struggle with a heavy door or lots of stairs to access therapy, as this can add to their difficulties, but such practical matters should not come at the sacrifice of the 'good feelings' in a house.
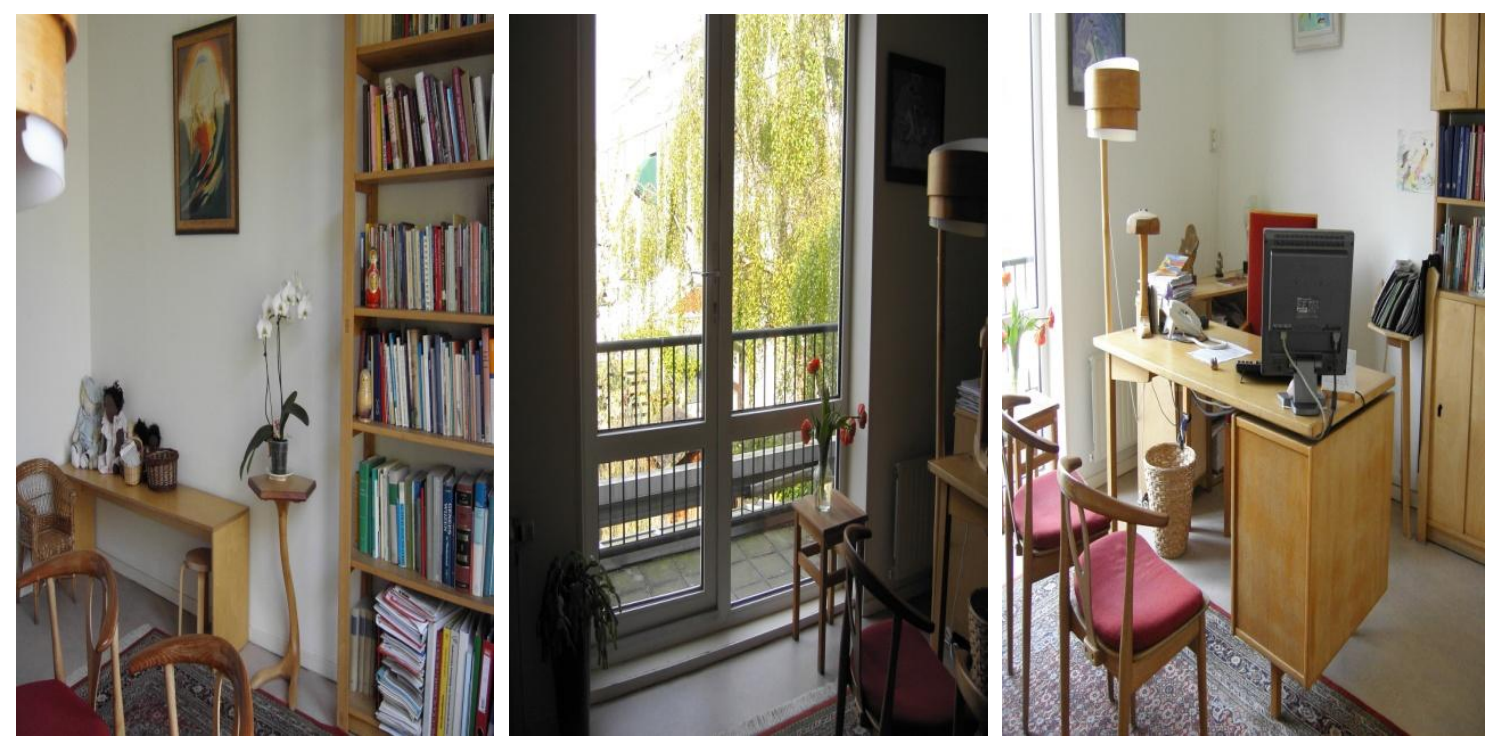

Triptych 7.8: General Practitioner 3's room at Site 2

This is a real contrast to the practice I witnessed in UK (who would also like a new site). There, it was all about getting the practicalities right and little about the 'feelings'. I have found modern GP care in the UK to be like this too, all practical and a nonymous - in fact I would love to have access to the care that Emil and his colleagues deliver. Appreciation of 'the personal' is really the hallmark here and this seems to underpin everything I saw.

It is very hard not to so und evangelical about this, as these people and place genuinely 'moved my spirit', much as their a pproach to therapy (and life) aims to. In a sense, I found here in Emil and his colleagues, everything that I had been searching for yet without ever knowing it. I found permission to be authentic and honest, I found appreciation for my personal worldviews and I found a community where I wanted to belong. I was finally 'normal', not once feeling 
frustrated at the limitations imposed by state policy or practitioner paradigms; these characters defied all of this which I recognised thro ugh being in contrast to (my) UK practice.

There, just getting an appointment to see a Doctor can take weeks, forcing patients to access locum or emergency care (hospital based). CAM practitioners, in many ways, can also replicate this, by having their set hours during which they will administer care to patients even if their telephones are more available (for example, if they work from home). The only exception I have found, within my experience, is my Homoeopath (which I detailed in my analysis of Nora). Maybe this is also related to the provision of being a whole healthcare approach (rather than adjunctive or allied therapies) being similar in application to anthroposophy, but whenever I have needed my Homoeopath (day or night) I have a way of contacting them and hopefully avoiding resorting to 'conflicting' treatment by mainstream medicine.

As a committed patient of Homoeopathy (which I freely admit to) I find having such access incredibly important to ensuring continuity of care and avoiding conflicting advice or regimens. Of course, respecting the boundaries of waking hours means often make first approaches by sending a text but know I can call, in extreme cases. The value and comfort this brings to managing my own and (more importantly) my children's health and wellbeing are truly beyond words. I was both surprised and pleased that Emil gives out his personal phone number to patients if he is concerned about their condition deteriorating over the weekend, so they should not feel compelled to seek conventional (suppressive) care in his absence. Like Martin, this was a sign of Emil willing to go 'beyond the call of duty' to bring the best of himself and his 'arts' to patients, which brings also an old-fashioned comfort to the trust and relationships between these characters - as previously evidenced by Nora. During 2014, I knew what it was like to not have this level of support and the implications this can have not only for physical health but also mental health.

The reason I raise this again is because it really crystallises the importance of continuity, in both practitioner and approach, that Emil provides and his patients' value. From Emil's perspective, when a patient has to access conventional care in his absence it leaves a lot of work that he has to undo, and this was the same for the new Homoeopath. This was something I recorded but did not truly value until I experienced the same thing, and the ramifications of that. In my case, knowing I have support going forwards markedly affects my confidence in the trajectory of illness episodes, and this is something that I strongly feel should be considered when assessing the long term outcomes and satisfaction of patients with CAM approaches. 


\subsection{Act 2, Scene 5: Healing by design}

Having explored some of my more memorable and striking observations from both Sites 1 and 2 I feel it is important to also examine Site 3, with regards to how those based here view and enact their schemes of care.

I found Site 3 a different centre to the other two sites in a number of ways. First, it is not a general anthroposophic/medical practice, but a dedicated anthroposophic/physiotherapy practice. The practitioners who work here are, therefore, not a diverse mix of healthcare professionals, but a small staff of specific bodywork therapists (specialising in the methods of rhythmical massage, bath therapy, meridian therapy and fitness rehabilitation). Second, it is housed within a modern, purpose built site outside the town centre, in a rural community along the river. Third, and most importantly, this combination means it uniquely meets all the practical needs and the feeling needs of those who attend here (practitioners and patients).

Elsa (not real name) is one of the physiothera pists who practices here. During my final week in Holland, I arrived early one morning to observe her delivering a treatment. My journey, along the river by bicycle, to the centre had again seen the relative hustle and bustle of the (small) City fade in the distance, along with any stress I may have been feeling. Replaced by the sounds of nature and farming, I was in 'another world' by the time I reached the centre and parked my bicycle outside.

Elsa was already there and we chatted briefly in her office area while waiting for her patient to arrive. When she did, Elsa and I got up and moved into the winding corridor to meet the patient, where she was retrieving her towels from the linen cupboard (it seems patients here, as with physiotherapy at site 1 also, provide their own towels for massage). I followed Elsa and her patient into therapy room 4, where Elsa quickly dressed the bed with a warm, yellow coloured flat sheet with the patient's towel on top.

Elsa looked casual and relaxed throughout the treatment, wearing a comfortable outfit of black leggings with a black vest top and short sleeved cardigan over. She guided the patient to lie face down on the couch (patient wearing only her underwear) and wrapped her feet, legs and lower back area in the sheet before putting heat bags on her feet and then placing an extra towel over her lower back and thighs. I sat on a stool near the wall and watched the sunlight stream over the pair as Elsa applied oil and delivered a rhythmical massage to her patient; first to the whole back, then upper arms and shoulders, then lower back and buttocks. Through this first part, social chatter gave way to silence from the patient and increased focus 
for Elsa, which I saw evidenced in her gaze, posture and breathing. Now the simple, bright and uncluttered space was silent, as a final move from Elsa to the sacrum generated a deep sigh from the patient. Elsa then asked her patient to gently turn over. Elsa repositioned the pillows to support her patient's head and re-wrapped the sheet around her neck, chest and body leaving the legs to be covered independently by towels.

While Elsa massaged each leg, the covered one benefitted from the warmth of the heat bags. Comfortable chatter resumed at this point, as Elsa delivered a range of recognised techniques to each thigh, calf and foot before recovering and wrapping in the sheet which was also covered with an extra (thick) blanket. For the final move, Elsa uncovered the patient's chest area, and using cream rather than oil, applied massage down the sternum and out towards the ribs, around the breasts in a circular direction. When this was done, Elsa recovered the chest with the sheet and blanket, confirmed the patient's next appointment in her diary and then washed her hands at the small sink, before setting the 'timer' and leaving her to rest.

I followed Elsa out of the room while the patient rested. Shortly after her 'timer' alarm rang, the patient emerged from the room, fully dressed, returned her towels to the linen cupboard in the hallway and helped herself to a cup of herbal tea from the caddy in the cosy waiting area. She sat there alone, quiet and still drinking her tea, and left quietly also when she had finished.

Reflecting on Elsa's performance here, there were many parts of it that looked like my own practice, with many similar techniques but less forceful, less deep. This was delivered with a different intent to my own, and also had a different quality, somehow, to the massage I had observed with her colleague here and also one of the physiotherapists at Site 1. Through analysing Elsa's subsequent interview, I found key markers that helped me to explore and then explain some of these differences.

\section{Elsa's narrative:}

I trained as a regular physiotherapist, upon leaving school. I practised in a number of different centres in [this City], and then quit working when I had my first child. After 6 years I met [Anthroposophic Fysiotherapist 3], and learned of anthroposophy. I was curious, I had always felt regular physiotherapy was missing something - I found it in anthroposophy. I took the 2 year training in anthroposophy, and began working together, with [Anthroposophic Fysiotherapist 3] soon after. Initially, this was in her former centre, but that was regular and noisy, so we wanted to move! I am a 
practitioner first, and defer the business role to [Anthroposophic Fysiotherapist 3]. I never intended to be my own boss, despite the benefits of this. I am more of a creative type and easily distracted. I do not work mechanically, and I integrate myself, into my work. Insurance regulations make it hard to practice anthroposophy. It is hard to demonstrate the quality and benefits of this work to the companies because they operate within different standards and philosophy; a different criteria. This path is maybe not the 'smartest choice'; it's not easy but we have an established referral stream from the Therapeuticum that supports our financial sustainability.

I really relate to anthroposophy, and am enthusiastic about learning. I do the rhythmical massage and movement therapy. The massage reconnects people with their body again, before exploring the qualities of moving in everyday life. It empowers people to react and choose how they use their body. We individually connect and dialogue with people on a human level. Everyone has their own struggles in life, I like being able to support and inspire them to make 'good choices'. This goes beyond my training, and is more of a unique quality than a learned quality. It is holistic practice; they are not just a number or a limb. In my former employment settings, I always wanted to respect the values of the management, inhibiting my self-expression. I think that knowledge and awareness comes with age. I have grown as a therapist because of my age and experiences. Uniforms can be a barrier and create disconnection so I do not wear a uniform, but I do think carefully about my attire. I am a person not just a practitioner, this celebrates my individuality. I do not like to be stereotyped in anthroposophy ways. I am part of this therapy, I am more authentic here.

We initially sought a central location because we felt this would be suited to more patients, but the property there was too dark, and not a good feeling. Finding this centre was like winning the lottery! This is a lovely location and the journey here makes you 'slow down'. We are only 10 minutes from our previous centre, and a catchall for this side of the City, and the surrounding area. I think it is a healthy environment, and really appropriate for therapy. It is nice to come here, I feel at ease in this space. Stressed people look forward to coming here. It has a quietness and lightness. The waiting area is like a cave, patients feel safe and do not always want to leave it. I link patient responses to the sense of safety and serenity here. I sometimes feel the therapy space is too small and restricted. I adapt to the setting, but I would like to have a little more space, for working with movement, and more equipment. My 
rooms, and use of space, have all been carefully constructed, staged and directed. The intention of this is primarily to benefit the patients, it is not a home only for myself, but a home for everyone! The soft walls, paintings, colours and crystal pendulum in the window are not practical additions, but homely ones! I do not really like the exterior, I would like to improve it with more plants and flags to blow in the wind, more beauty! I want to change the entrance, I do not like patients using the back door. The dogs, seeking attention, is a disturbance and a distraction when trying to do paperwork, or make phone calls.

It is important to like where you work. Where we were before, was not good. It was on a busy street corner and had a gym upstairs. This made it noisy, and you could hear the consultations. I used to feel more tired there, although I tried not to let this rule the treatment. I am self-aware about the impact of place and space. Here, I feel more energy and vitality. The lack of distraction in this setting is good for therapy, and good for me. I feel this benefits patients also, they come here because they are out of balance. I would like greater contact with our colleagues, it can feel a little alone, or isolated here. We have to make efforts to share dialogue, which costs time, by phone or drop by. Connection with space is easier in your own space. Space is part of therapy, and everything makes a difference. It has to feel right, not just look right. I do not like the 'cloud' effect in every internal space, we have it on the floor instead of the walls.

Elsa is another character with sensitivity. She was quite 'late' to anthroposophy by some standards, having been neither raised nor educated in the philosophy from childhood. Still she managed to find and make this 'her home' anyway, when a chance meeting resulted in her working alongside a physiotherapist who had also trained this way.

In a sense, this 'awakening' is similar to my own. I originally trained as a 'mainstream' remedial massage therapist and although I felt biomedical philosophy to be limited, did not really know how else to view things. Then, when I found myself undertaking a CAM degree (to seek academic validation for my therapy and the opportunity to grow within NHS provision) I began to 'see' where my natural views and affinity lie. Like Elsa, this proved an important part in my development, underpinning the practice I deliver today (both therapeutically and academically). Elsa, too, relates her training, life and experiences to the nature and quality of the therapy she provides, putting herself into her therapy at every possible point (including her attire) as expressions of her own force and identity. 
This recognition of individuality in herself (as a practitioner) may also underpin her recognition of heterogeneity in patients and make it possible to connect with them on a one-to-one basis. Like she says, "they are not just a number or a limb". Neither is she anonymous ("I am a person not just a practitioner") and I saw her actively use herself, during therapy, in this way; bringing herself and her experience into the treatments, as others here also did.
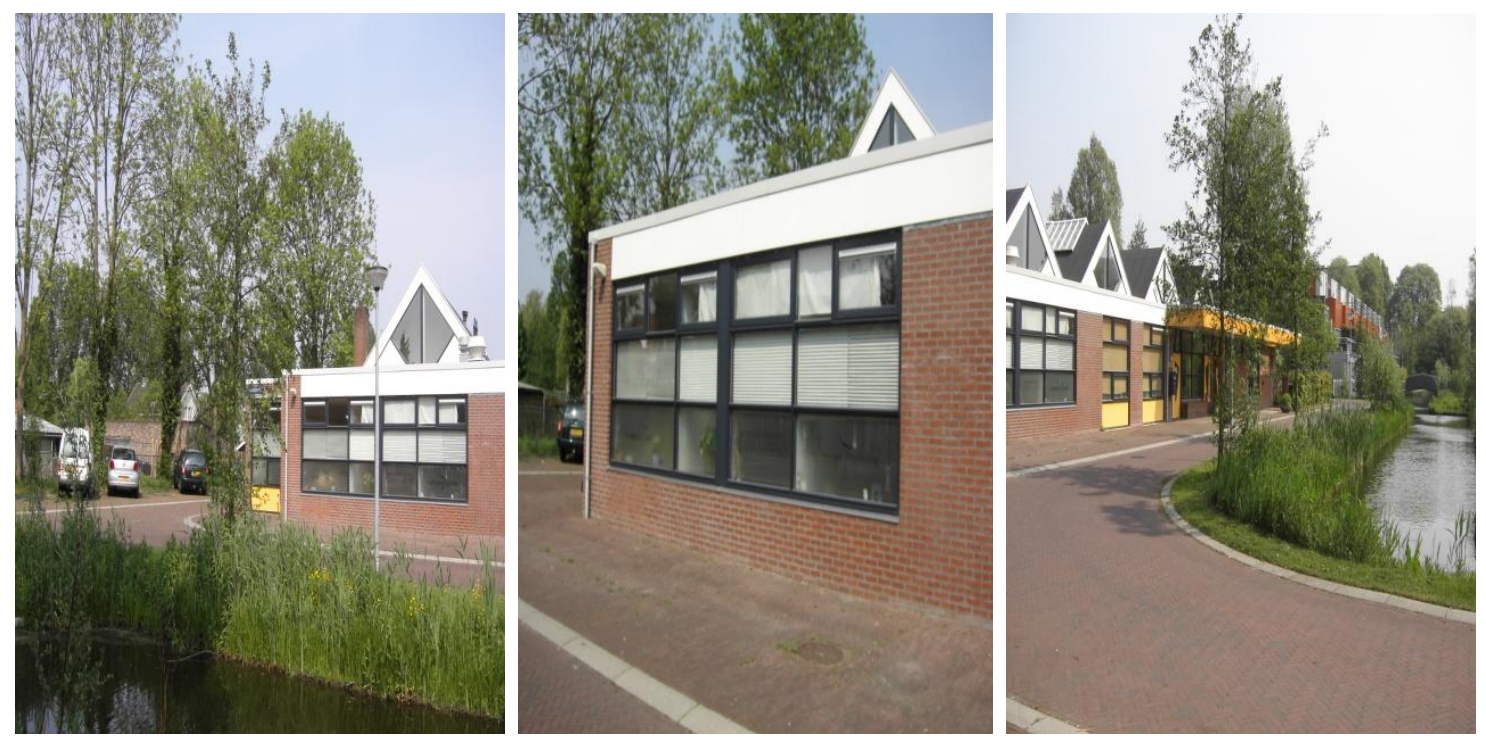

Triptych 7.9: The exterior façade of Site 3

I am not suggesting this is unique to anthroposophy or this setting, as indeed practitioners at the UK site suggested similar applications, but here I felt the practitioners seemed more aware and connected to the fact they are doing this (and that this is in line with their training rather than contrary to it). Elsa also appears to connect to her surroundings and place of work in this way, 'in tune' with how she feels here and then transmitting this feeling, through her interactions, to patients. I learned that, along with her colleague here (anthroposophic fysiotherapist 3) she also played an active role in the design and creation of this centre, agreeing to rent the space while it was under development and so influenced the room sizes, 'curvy' corridor, natural lighting, textured decor and practical fittings. This was an important, further level of connection with her space that means she feels to be the best supported she can be (so no 'frustration' like Simon, in chapter 6). She is relaxed and at home (as Emil has also suggested) and finds this also makes a difference to how relaxed and at ho me her patients feel. I would go further than this, and suggest this quality of engagement also impacted how relaxed and at home I felt here. I will reflect later on the difference this made to my data collection and data connection. 
I spent a good amount of time in the company of the two therapists who run this site, independent yet linked to the others by referrals. I also observed several treatment sessions and interviewed several patients alongside these but perhaps my most unexpected finding came not through observations or interviews but through receiving an example of this 'unique' style of massage with one of the physiotherapists at Site 1.

I had observed treatments both with therapists at Site 3 and Site 1 and knew that when I received one of these it was not going to be the deep, forceful treatment I am used to delivering (and receiving). I found it pleasant enough and appreciated that any potential is intended to reach its full effect over a course of sessions, being not only a physical therapy but more importantly one that (through the oils and techniques used) will 'speak' to the body on many levels. Therefore, my experience was never going to be a 'complete' one, however I did find it most illuminating.

As with those I had observed, my own treatment had been gentle and rhythmical, concluding with a 15 minute rest period (during which time my body would begin to respond to the treatment) and then a cup of herbal tea to refresh and reconnect me to a wakeful state. On rising after this I felt 'wonderful', more wonderful than I had expected given the nature of the massage compared to what I a m used to. As I tried to make sense of this, I recalled something from my training days regarding aftercare advice. Indeed, something I had continued to implement all these years was to advise for patients to rest and take on fluids after massage, to help the body respond and process the effects of the treatment. It was something that patients hardly ever followed, often too busy getting back on with life, and so I likely never realised the benefit this could have. But here, I was forced to rest for 15 minutes and then offered a drink of herbal tea before leaving, and for the first time I realised the value of this!

Observing and interviewing only, while noting this happened and patients liked it (patient accounts always mentioned 'the tea'!), I never comprehended the full impact these two parts of the treatment actually have (and they are parts, not extras) on the experience of this care. Sharing the experience helped the value to connect with me in a more 'real' way so that, like these patients, I could see just what a difference this ending to the treatments makes. This also brings back an observation that Nora made (previously) in regard to the unique role of personal experience in appraising and engaging with different therapeutic approaches. When I had not experienced exactly what these patients did, I could not identify or value any of these aspects as they did. Once I had, however, I was better placed to represent these experiences and these people. 
I did not think to explore this nuance at my UK site, as they practiced much as I did (aftercare advice only) in many aspects of that service. While I did notice some differences, I cannot give these perhaps the gravity they deserve which is an omission of method, based on prior lack of knowledge and appreciation (this is quite ironic, as this is a point I have sought to explore within this scene). Here, I was less educated in both the philosophy and the practices and this made a clear hole in my ability to appraise fully everything I witnessed. Maybe this, more than the development of a new methodology, is the change researchers need to make in order to enhance their design and reporting of trials and outcomes. After all, this is how many of the ancient and CAM modalities used today were developed. Certainly, my experience here added something critical to the mix that had obviously (although I was unaware at the time) been lacking before. This is something that I hope research can take forwards, from my data.
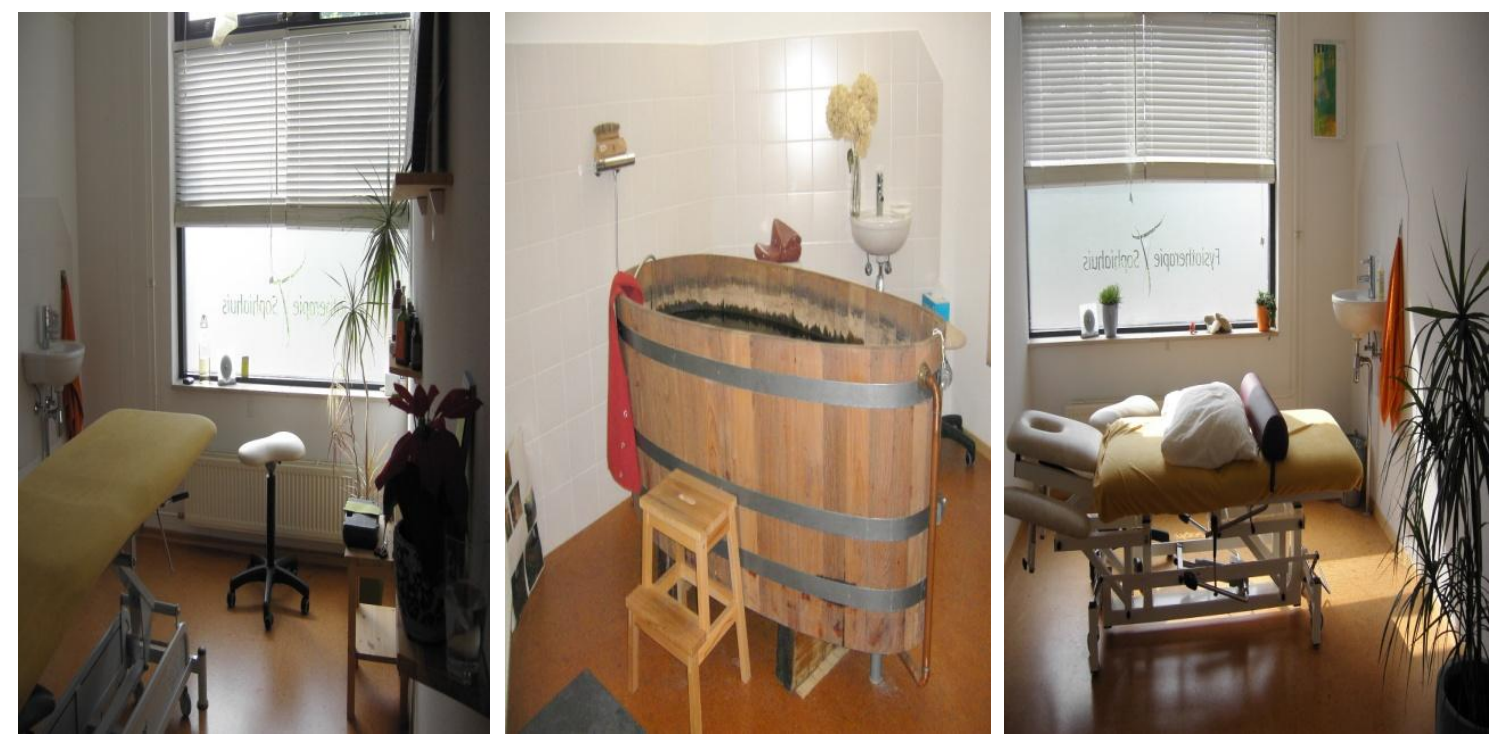

Triptych 7.10: Physiotherapy rooms at Site 3

A further important point, that Elsa raises, is the issue about insurance payments. This is the same issue that I was keen to highlight when I began this study, exploring the compatibility of therapists' aims or intentions with the intentions and aims of researchers. From my review of the literature (in Chapter 2) and experiences gained at the conference I attended in Berlin, it seems there are many examples of incompatibility in CAM practice and research. This may be a fundamental matter in need of attention, to enable the validity of such studies to improve. Potentially an issue of funding, comprehension or even expertise, any lack of 'space' for researchers to research therapies 'as they are intended' presents a real problem for quality in CAM research. This may be 'how it is', but the acceptance of the status quo must not 'throw the baby out with the bathwater' and if the pull (or promise) of evidence-based medicine has 
created a need or desire to fulfil external, societal or academic requirements above thera peutic ones, then this must also be challenged.

The experience of inhabiting two worlds is a pressure that I have faced within my research (discussed in Chapter 6.1, in regard to conflicts between my philosophy and the UK practitioners) and so I identify with some of the challenges this also brings to Elsa. Just as I have struggled to develop and perform a style of communication about my findings that is accessible to the reader, so Elsa and her colleagues face this constantly and will continue until such time as the external discourse surrounding them develops to acknowledge their holistic and sensitive methods and outcomes. The amount of care alone these people must show when completing and submitting insurance claims, to select wording or language that is compatible with the ideas and discourse of the insurance companies, shows that there exists a chasm between these. This chasm also underlines Nora's observations about her insurance status, which necessarily influences her access and experience of this 'medicine'.

For practitioners here, this conflict is (at least) balanced to some degree by their practice and patient community, almost all of whom try to live their lives within the whole philosophy or teachings of anthroposphy. This means that when they slip back into this unique reality, they feel supported rather than isolated which in turn strengthens them whenever they do have to face those external challenges again. I feel sure that this kind of environment would have benefitted characters like Simon (in chapter 6 ) to be more true to himself, in his practice, as it would have me, in my research.

More than anything, I took from Elsa's performance here, and others I witnessed that interconnectedness between place, people and plot really makes a difference to how these therapists deliver, which is the factor that mediates what these therapists deliver. Influences on this can be cultural, political, spatial or social but being in this place, with these people really brings the whole matter to life! The 'parts' are important because they are 'parts of a whole', from the peaceful location and the freedom to reject 'uniforms' to the ability to be creative and defer all business and administrative matters to [anthroposophical fysiotherapist 3]. This all makes a difference; nothing more and nothing less.

\subsection{Act 2, Scene 6: Coming home}

For my final scene here I want to explore the story of another influential character, who inhabits both Site 1 and Site 2, while also acting out in this 'community'. Marianne (not real name, anthroposophical nurse) is a lady whom I got to observe and converse with at many 
points over my time here. She enacts a number of different roles within the practice including the rubbings/enveloping therapy, social care and advocacy. Again, she never dressed like a nurse, and today she was wearing loose beige trousers and a 'flowing' white shirt. Like Elsa, Marianne too came to anthroposophy as an adult, soon also finding this to be her 'spiritual home'.

It was during my third week on site that I conducted the following observation of a rubbing/enveloping treatment with Marianne. Marianne and I were together in the Nurses suite on the second floor of Site 2, when her patient arrived up the stairs into the kitchen/entrance area. She and Marianne exchanged some words (in Dutch) and then we progressed together into one of the small therapy rooms.

I sat on the shelf by the window and watched their ritual, as the patient removed her clothes, keeping only her underwear, and climbed onto the therapy couch laying face up. There was already a sheet draped over the couch, which Marianne wrapped around the patient after first covering her chest with a small towel. She then positioned two pillows under the patients head and a bolster cushion under her knees. In a calm, relaxed and methodical manner, Marianne then proceeded to apply the oil which had been prescribed by the patient's Doctor, in rhythmic strokes to specific areas of the patient's body. She sat the patient up and uncovered her back, applying oil first to the right side and then to the left side. The patient then lay back down and Marianne uncovered her arms, applying the oil to the forearm, hand and upper arms; one arm and then the other. Next, she uncovered the left breast area, applied the oil and recovered; then the right breast area and recovered. Marianne now moved a little down the couch to uncover the patient's abdomen, applying the oil across the body and recovered. Finally, it was the legs; first the right thigh, knee and lower leg, then the left thigh, knee and lower leg before concluding with the feet, each covered in the same rhythmic strokes of oil, before recovering.

This whole episode was so quiet and calm it appeared like a meditation, for all concerned. When the patient was all wrapped up again, Marianne left the room briefly to fill a hot water bottle in the kitchen area, which she then placed under the sheets in a space between the feet. Marianne then checked the patient was fully wrapped and warm beneath the thick yellow blanket and we left the room, for her to rest and absorb the therapy. The patient stayed like this, enveloped, for 20 minutes after which time Marianne returned to her with a cup of herbal tea. The whole thing looked like having a treatment in your own bedroom, at home. 
A unique service but a regular part of Marianne's work here, this is one of her favourite things to do. I learned more about her journey into this role and her life before and around this, during a most candid and enjoyable interview.

Marianne's narrative:

I always wanted to be a nurse, from a little child. I only knew regular nursing, so this is what I trained in. I worked in many different areas including psychiatric nursing, regular hospital and special needs particularly Down's syndrome. I liked the mental health and community nursing, but hated the hospital nursing. After a career break, to have children, I returned to district nursing. Then I got my divorce and had the 'rubbings' with [Anthroposophic Nurse 2]. I was not trained in anthroposophic nursing and I remember saying that I wouldn't like to do this, but a year later I was working here. I really loved the course. Anthroposophy felt like coming home! I would have been a Steiner teacher if I had not become an anthroposophic nurse. My oldest son is a friend of [Anthroposophic GP 1's] oldest son and he personally approached me to do this. My children attend a Steiner school so I have known of anthroposophy for 15 years. I spend around half my time delivering the 'rubbings' or 'enveloping' therapy and the remainder 'talking' with patients, for social and psychiatric problems. I also do things here like taking blood pressure and injecting anthroposophic medications.

I am employed by the Doctors, and not concerned with running my own practice. Originally, I had a portable therapy table either in the little office downstairs at [Site 1], or in the room of [Anthroposophic Psychologist 1]. I really did not like the small office, it was a cramped space with no natural light. Then we got a permanent space in what is now the library at [Site 1], but really that was too small for 2 beds. We moved into this space [Nurses suite, Site 2] when the previous, private tenant moved out, only 5 or 6 years ago. It's a really great room, and my favourite place to work. I love that I can see trees and the Cathedral from my work space. Three of us share it; we created it together and chose everything from the kitchen table to the rug and the 'talking' chairs. We each take responsibility for different parts like a family, for example the flowers, the laundry or the groceries. I like a centre to feel like a home. It is very peaceful, quiet and with a totally different atmosphere to [Site 1]. Space can help or hinder. I could not work at [Site 1] 3 days a week; it is too noisy, distracting and disturbing. There are lots of practitioners, lots of patients, lots of activities, energies and problems, and these remain in the building. It has been a practice for 40 years! I 
can sense 'spirits' there and never like to be alone: you never are 'alone' there. A new centre would be a practical approach, but I like a smaller building, with fewer people and the intimacy of a small team. You can be quiet in yourself here. A large centre can be a negative influence if it is too busy, or too noisy. Hospital settings are also in too much hurry, there is no time to 'be' with patients. I love that I have the flexibility and the freedom to give each patient a full hour, many social psychiatric nurses are only permitted to spend half an hour with patients. I can do a better job because I can give time. I feel very supported and encouraged here, I have autonomy. It's a great feeling. My model and pattern of work is calming to do - it's a good counter-balance for a hectic life! I could not be as effective in a different routine. My way of working comes from within, I have my training but I do it my own way! I like to 'be mother' and put myself into all my work. It is important to have time for people. A rushed start to the day would adversely affect my work and not be good for the patients. I like to do the 'rubbings' in the morning to be busy with my hands and empty my head, and also to end the day with this, and have the 'talking' in the afternoons. This work is not strong, it is not massage. Sometimes I will do 'rubbing' on a home visit, if people cannot get to the centre, or use the stairs. I like to work at people's homes, to 'feel' how they live. I am sensitive to energies 'with people' and this helps me to help them make changes, to get better. Everything has to fit, for my best work. I feel permitted to be caring here.

Of course the place impacts both myself and influences my work. It is not possible to separate them. A family, home setting helps the practitioner, and I think this atmosphere impacts patients also. My best moment of the day is morning coffee at [Site 2]. We all meet in the little kitchen and share each other's news. They tried this at [Site 1], but it never succeeded. They are a mix of employed and self employed practitioners at [Site 1], whereas here [Site 2], we are all employed by this practice. We are more connected and that makes it easier to take the time out. Everything is so important, and yet not valued. It's funny no one studied this before -we all know it!

Again, Marianne is a sensitive soul. She was drawn to this work by life events, but opened herself to its wisdom and came to adopt not only a new employment but also a new lifestyle. Hers was perhaps the most similar journey into therapy as practitioners in UK (experienced it, and it helped, so now want to help others), which means it was also the most similar experience to my own. 
This journey was serendipito us, in many respects, which itself reveals a direct link to the whole philosophy and practice of this form of medicine (anthroposophy), whereby everything is interconnected. Many may interpret this as 'chance' but for Marianne, like others here including myself, the links are there if there is a willingness or just openness to look for them. Making meaning from these relationships is also a form of 'meaning-making' to give her life and work a coherent purpose. This is not to say I felt she 'forced' anything that was not there, but that in being open to there being relationships, she found them.

I felt this was rather like my practice in therapy, when patients say to me 'oh, you always find the right spots' and my response is that I simply go looking for them! This is the approach I have tried to re-enact throughout my research also, aiming to discover what a 'practitioner's eye' (so willing to perhaps look deeper or further than regular researchers) may reveal that had previously been overlooked. But this is not a totally unchecked method. For Marianne, it is based upon a solid education and foundation in anthoposophic philosophy and teachings. This guides her in a similar way to how my clinical training guides my clinical practice, and my University education coupled with theatrical training has guided this research.
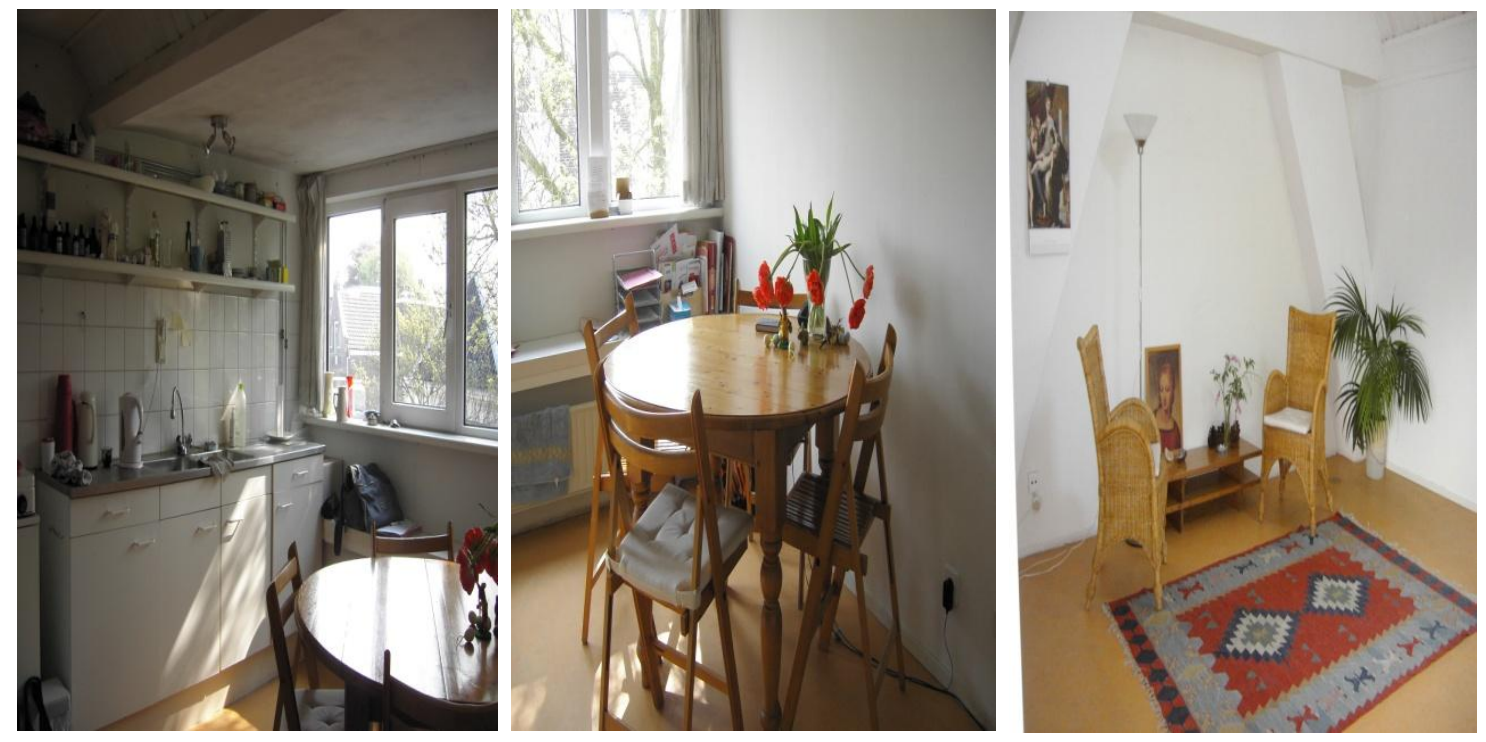

Triptych 7.11: The Nurses Suite at Site 2

I therefore did not question Marianne's method, but simply recorded that she actively engages and incorporates this into every aspect of her being, exhibited through her work and social relationships. She was very clear with me, that she could not do this work without the philosophy present. This raises again the issue of validity in CAM research. As Marianne states, "Everything is so important, and yet not valued. It's funny no one studied this beforewe all know it!'. This is perhaps the clearest evidence of the 'research-practice gap' that I 
spoke of earlier. I had identified it within my own practice and seen how this differed from the UK site; I had also listened to the many stories of participants here, across disciplines within a large group practice; and now Marianne was telling me directly.

Taken within herself and 'made sense of', Marianne was an embodiment of her art and this made her more than a method of delivery. She uniquely enhanced her delivery much as others here also did - the main difference between here and the UK, in this respect, was that practitioners here seemed aware of and embraced the opportunity to do this, to be active in their care, whereas in the UK the individuals often tried to 'play down' their role (while acknowledging they do have some influence). Again this gives rise to conflict within individual characters, limiting their 'space' to make potential relationships. Without such inhibition, it became an honour for Marianne and the others to 'carry' these patients, until 'they can walk' again.

Concerned that I should 'know' her therapy to report on it adequately, Marianne insisted I allow her to provide me with some enveloping therapy, while I was here. Martin prescribed what he felt may be an anthroposophic oil for me, which Marianne applied during my treatment. In a practical sense (at least) I felt knew what to expect, from previously observing Marianne with her patient. The whole session felt calm, although I chattered rather more than most do (in fact Marianne asked me if I ever liked to be quiet!). Marianne applied the oils to me in exactly the same way she had the patient before. She then wrapped me and placed a hot water bottle under my feet, and said she would leave me to rest. It took a little while for me to open myself to any possibilities with this therapy; I wanted to, but it was so new and alien, I just didn't know what to expect (which is ironic as by this time I had already observed and interviewed several patients, who had experienced this treatment, and spoken to me about it).

As with the rhythmical massage, I hadn't realised how powerful this treatment really was until I experienced it, first hand. Just the silence and the peace (inside me, more than outside) was remarkable, but my therapy was more than this. It was also caring and inviting, a sort of 'invitation to be'. Sure, this felt awkward at first; I looked at the peach, cloudy walls; the peach, cloudy ceiling; the trees through the window; and listened to the birds singing (this was the same room Nora had her infusion in) all before I felt ready to close my eyes and embrace the opportunity (creating in myself a 'space' for potential relationships to occur). This was when everything started to get weird. Behind my eyes a dark, purple cloud began to descend into my upper chest like a midnight storm. This was followed by another, then another, then 
another. This continued for a long time (or what seemed like a long time, but was probably only 5 minutes) and then there was nothing except a kind of stillness, that was still dark purple. All of a sudden I began to get the feeling of a 'darkness' lifting from me, almost as it had descended, cloud after cloud after cloud rising up from my chest, up towards the ceiling and then away.
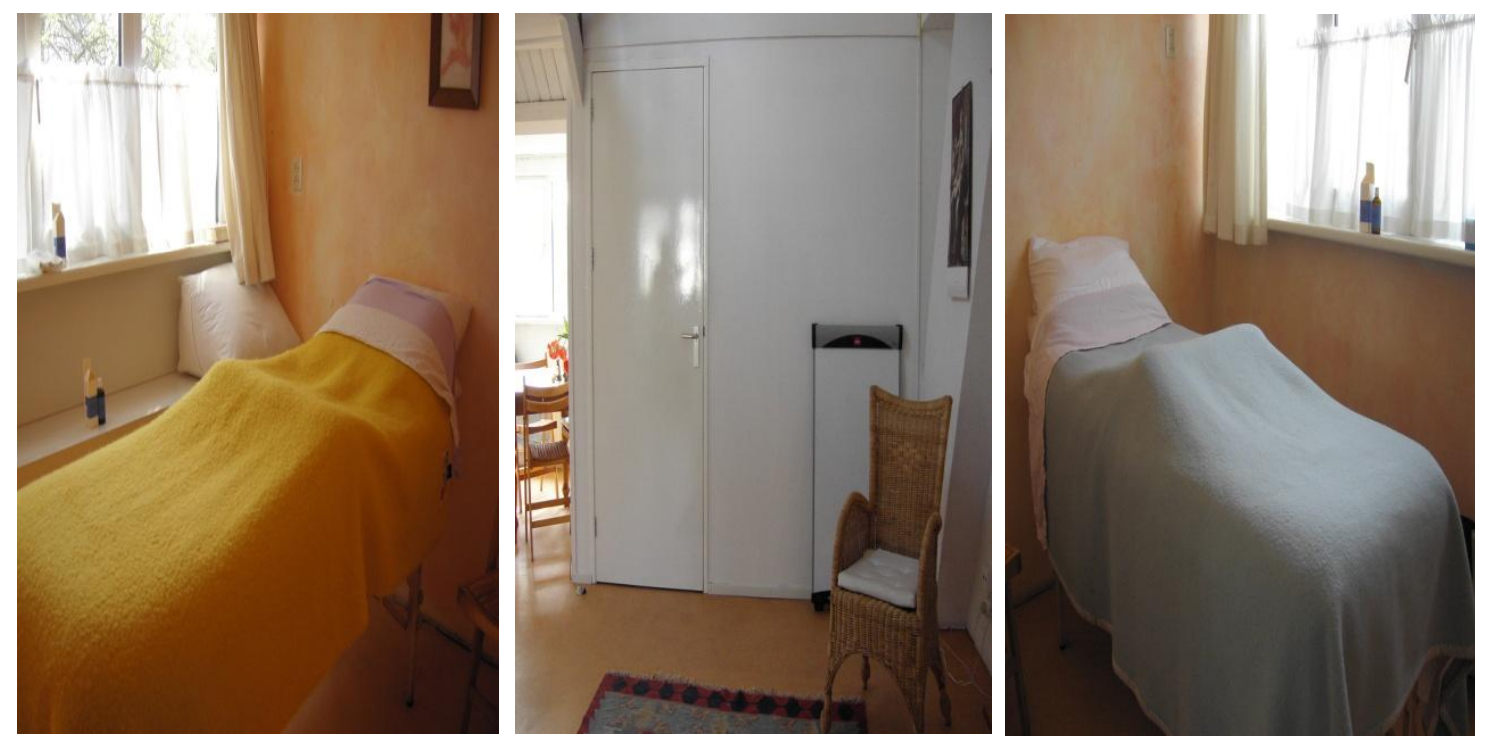

Triptych 7.12: Therapy rooms within the Nurses Suite at Site 2

My eyes remained closed through all of this as, having given myself to the treatment, I did not want to short-change the outcomes. I was aware of nothing outside of myself and these visions, yet I knew I was secure and felt safe here. I trusted Marianne completely, and because I felt that I could, not because I felt I should. I do not know how long this state (or indeed whole experience) lasted but then a brightness came into my vision, and I felt encouraged to open my eyes. Marianne was standing at the side of me with a cup of herbal tea. She set it on the window sill and asked me how I was. I told her: fine, weird but fine. After Marianne left again, I laid for a few more minutes reflecting on what had just happened to me, before dressing and joining her at the kitchen table. She checked I felt okay, and then we went downstairs together, to join the other staff for coffee in the tiny kitchen. They seemed to know that I had just engaged fully in their process and were all interested in hearing my experiences. I felt closer to them all, as a result.

Again, I possibly didn't recognise this at the time, but taking part in this experience actually provided me a whole new level of insight and meaning to the data I had gathered here, about the place, the people and these plots that I was otherwise missing. Most importantly, I began to feel the interconnectedness of things that participants also often reported feeling here. For 
example, feeling comfortable and settled here (with the people and the space), Marianne delivered a very comfortable experience for my 'rubbings' which I also felt comfortable receiving (matched my holistic expectations). Little touches also, like the herbal tea, ever changing table and flower displays (to connect the seasons - as Jenny had once commented to me people "do not always realise what time they are living") only added to this; sometimes the effect was subtle, and other times it was more powerful.

Of all the people I met here, Marianne was one of the most memorable, for me. Perhaps mostly, because every conversation I had with her was like having a conversation with myself. I felt I shared her worldview, her style of engagement and her intentionality. Either that or I saw in Marianne how I would like my worldview, engagement and intentionality to be. I would certainly like to feel as fulfilled and balanced in my work as Marianne is in hers, with the support and conviction of all around her in a blissful congruence with her own, genuine love.

This was not my final experience here, but this is the one I have chosen to close this performance with. For me, there was no better example of the problems within current CAM research and trial designs or a potential solution to this.

\subsection{Act 2, Scene 7: Critiquing the whole (holistic) therapy performance}

In a contrast of feeling and aesthetics more than amenity, I found this location to be a seamless blend of beauty and nature, resulting in a performance of therapy rich in movement and harmony that 'lifted my spirit' at almost every turn.

Presented as a coherent service across the City, this single 'group therapeuticum' is actually the joining together of three distinct practices and the practitioners within. Site 1 , the largest, is located in the north of the City, close to all major travel routes, train and bus stations. Site 2 sits in a more residential street in the shadow of the historic Roman Catholic Cathedral, to the west of the city. Site 3 , in some contrast, is located outside the City, to the south, in a landscape of rural farmland and large waterways. Across all three sites, the therapeutic approaches within are applied under a single, shared philosophy that sees mankind as an interconnected whole of "physical body, etheric body, soul (astral body) and Ego (Spirit)" which can only be healed by applying "remedies that can restore the balance" (Steiner and Wegman, 1925:17). Thus the overarching intention is of reconnecting people to themselves, each other and their wider environment.

I experienced this presentation and performance as an example of what 'real' integrated medicine could (or should) look like. A holistic ethos and non-invasive/natural treatments 
provide the first line approach, which aims to re-establish balance within the whole person, while conventional diagnostics and pharmaceuticals provide a back-up (crisis) service. This is not the picture of co-option that Doctors and researchers in Berlin seemed to either envisage as the 'best of both' or regard inevitable if CAM seeks acceptance by the masses. Instead, it takes the fundamental elements of both systems (CAM and conventional medicine) and finds a way for them to exist beside each other, in a position of mutual respect.

Here the only compromises come from making these services 'fit' within their built environments or negotiating legal regulations and restrictions. Unlike the UK site, there is no compromise in the style of performance to fit societal norms or economic pressures. Binding the many unique and wonderful contextual variables into a coherent and healing display, the services and sites that characters deliver across these 3 locations is not just an education in healing, but a lesson in living that offers a different and deeper dimension of delivery and experience that is truly holistic. Embodying the connection of the human spirit to the world (and people) around it, every event becomes an opportunity for development, every interaction stimulates movement, and even just watching or talking to these characters enables the spirit to grow.

In terms of evaluating the general and specific approach to practice, here practitioners share a clear and complete philosophy, that provides a blend of art and science somewhere between regular (allopathic) and homoeopathic medicine, seeing this are the 'right measure' to bring people back into themselves and back into health. It is an intended salutogenic approach, not a suppressive or palliative one. Part of a larger, coherent movement, they also demonstrate close ties with all things anthroposophical from schooling to the arts, the presence of which, in their immediate and wider community, provides succour, support and interconnected wholeness (healing). In this, practitioners claim to feel as human and carried as their patient's do, which I saw evidenced in not just how healthy they appear but how happy they appear. Enacting this model of care therefore seemed to me as therapeutic for these practitioners as it was for these patients. Engaging here actively, as I did, I felt this too.

This setting, therefore, made an absolute difference to the experiences of therapy (and my research), creating a production where practitioners and patients both actively acknowledge the healing potential inherent in the landscape and also engage with it on a whole person interconnected level, whether in design or in living. I found this a marked contrast to the performances in the UK, where the place was valued primarily for its practicality. With a similar impact on me also, my cognitive dynamic and subtle energy levels all came to agree 
with these characters, that no aspect of this performance either can or should be overlooked, whether evaluating practice, patients or research.

Characterised also by 'sensitivity', evident in the settings, plots and people, this constant theme was one that all characters actively contributed to and shared in. From practitioners to patients, a consensus of philosophy congruent with anthroposophy, saw the different elements of physical, etheric, soul (astral) and Ego (Spirit) actively sought and engaged. As an underpinning to this performance, practitioners were seen to act with sensitivity which patients eagerly welcomed. This was a different dimension of performance.

\subsection{Act 2, Scene 8: Reflecting on my research performance}

As my first overseas field site, this could (or maybe should) have been a challenging 'leap' for me to take, yet I actually found this whole engagement very easy. From my first welcome and the enthusiasm and interest from participants to the care extended to me in my treatments, I felt embraced, connected and carried through my various research roles and enactments. So powerful was this level of connection that I felt it even when I was back at home, either listening to the audio interviews or even writing up the findings in this chapter.

I gathered many additional positive experiences here, which also influenced my participation in this culture. These included trips out with my family (on days when I could not 'work') like celebrating Koninginnedag (Queen's Day), where the entire City became a giant flea-market, filled with parades, live music and colour (people all wear something orange); we also took a trip to the beautiful (tulip) bulb-fields at Keukenhof, enjoyed a couple of delightful river cruises and even escaped for a day at the beach. These events, and the lifestyle they supported, contributed to so much positivity that perhaps I found it more difficult to identify any problems or inconvenient issues that I would otherwise? But then, my story was also the story of these participants. They live here, they love here and their reports were all equally positive. I also found it very refreshing to be always around others who were looking for the connections (or interconnections) between things - which I felt made it easier for me to do this, also. As with the therapies I witnessed, my acts here appeared subject to these positive influences from people and place. This prompted me to wonder whether the opposite could occur - could always being around people who do not seek holistic connections, but pick apart their observations and findings, make it harder to see or achieve holistic aims?

Certainly, once back in the UK, I found it much more difficult to continue this. Handling the data I had collected was much harder than it had been to generate this, a task that was 
additionally complicated by my desire to give everyone (who wanted to use it) a voice. The sheer volume of data I was left having to organise and analyse was a challenge that got harder, not easier, the more time I spent doing this. The enthusiasm and interest of participants coupled with my own passion for this place and these people made it so that, for a long time, I really found it impossible to 'reduce down' the content to a level where I could effectively manage it and a reader could effectively comprehend it. I suppose I feared that doing so would automatically reduce the richness and quality I had uncovered. The opposite, of course, turned out to be true.

Realising this one, simple fact and learning how to 'highlight' the richness within has been and remained one of my biggest challenges, throughout the writing of this thesis. I don't know why this should have been the case, except that somewhere, between collecting the data and coming to present this, I lost sight of the 'essential' things (to quote Emil) and could only see the 'every' things. A bit like one of the issues that trouble CAMs (being defined by what it is not, rather than what it is) I suddenly only knew what I did not want to do, rather than what I did. I knew I did not want to pick apart my engagement with this whole performance which created so mething of an identity crisis for my study.

The critical, missing ingredient from all the connections I made in the data and dramatisation, turned out to be me. In including everyone else I had written myself out of the story! When I started to write myself back in, I began to identify the essential things as I saw them and this is what I have tried to communicate here, through connecting myself to those participants who made either the most unique or most striking impact on me. I still remain quite awe-struck and feel privileged to have spent this time surrounded by people who seemed to genuinely see the world the same way I do. They made me feel more at home that I have ever done and this is very special. I would love to return here again. 


\section{Chapter 8 "Resurrection Man"}

For my third therapy setting, I travelled to the sunny island of Cyprus, in Southern Europe. This setting was unlike any of the previous and offered a true escape from the outside world, surrounded by lush green gardens, a private stream and uninterrupted peace and tranquillity. The epitome of a 'healing centre' it is both geographically and philosophically as far removed from mainstream medicine as I could conceive. Here, headed by a senior Holistic Practitioner who is supported by two assistants (one also a student naturopath), one receptionist and an external Osteopath, 37 different complementary and natural modalities are offered as a collaborative performance.

To aid a sense of the physical space, set plans are as follows:

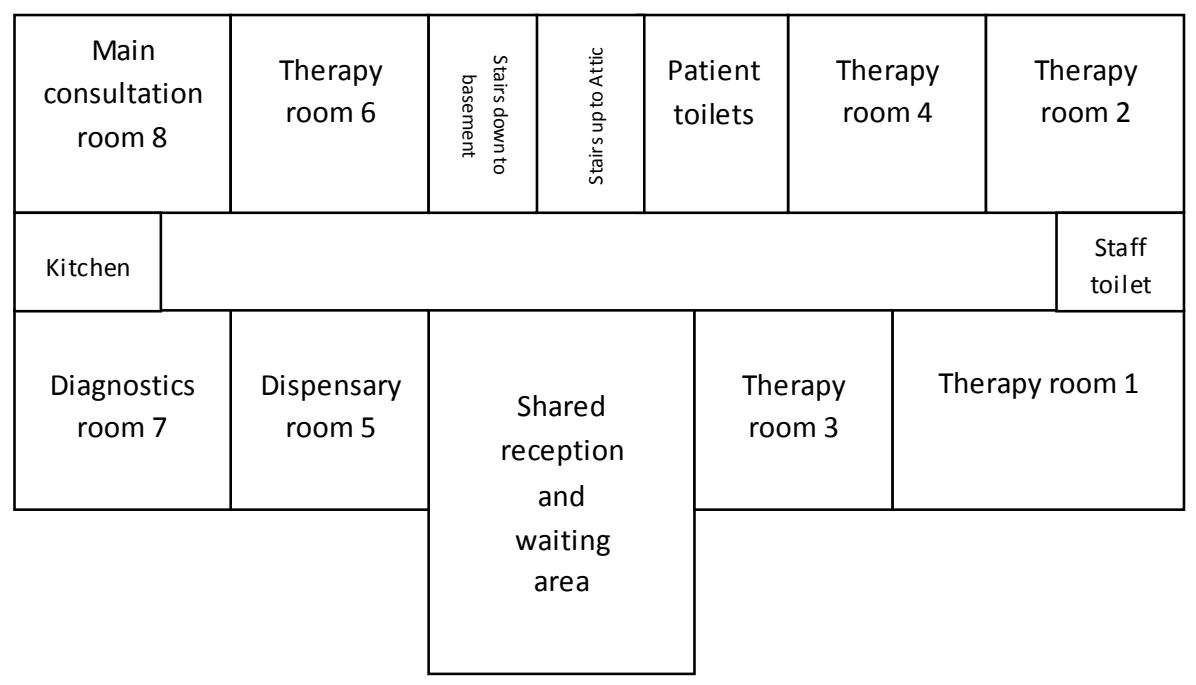

Set plan 8.1: Ground floor

\begin{tabular}{|c|c|c|c|c|c|c|}
\hline $\begin{array}{l}\text { Unallocated } \\
\text { room }\end{array}$ & $\begin{array}{l}\text { Osteopath } \\
\text { room } 6\end{array}$ & 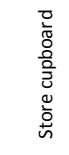 & 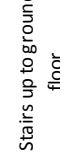 & $\begin{array}{l}\text { Patient } \\
\text { toilets }\end{array}$ & $\begin{array}{l}\text { Unallocated } \\
\text { room }\end{array}$ & $\begin{array}{l}\text { Unallocated } \\
\text { room }\end{array}$ \\
\hline $\begin{array}{l}\text { Unallocated } \\
\text { room }\end{array}$ & \multicolumn{5}{|c|}{ On-site Health Food Store } & $\begin{array}{l}\text { Unallocated } \\
\text { room }\end{array}$ \\
\hline
\end{tabular}

Set plan 8.2: Basement 


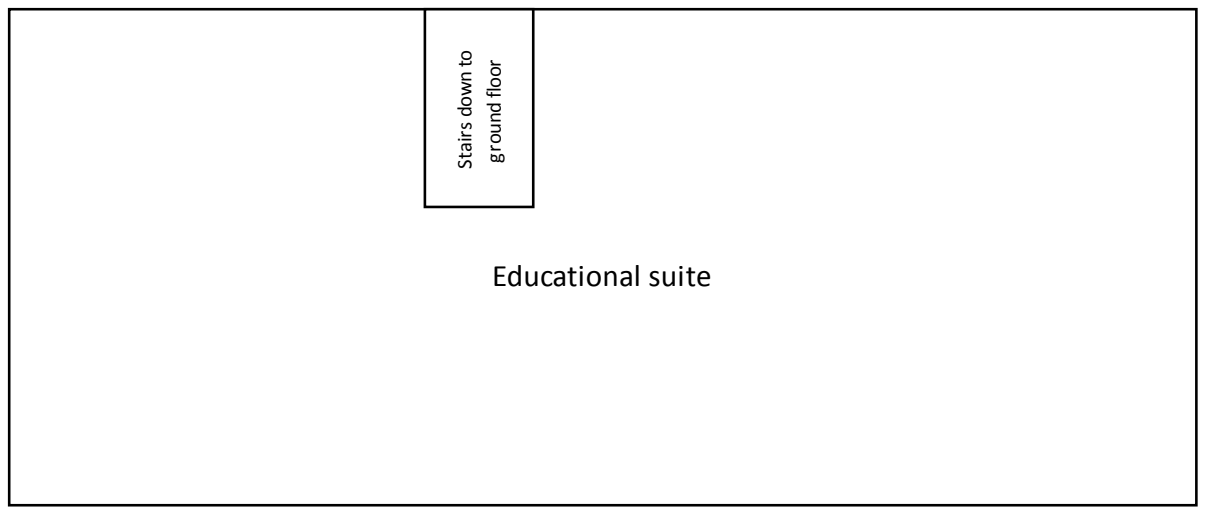

Set plan 8.3: Attic Level

I spent three weeks on site here, and during this time conducted one 'full morning' therapy observation, some general observations, also completing four practitioner interviews and thirteen patient interviews. Together the interview data totalled 15 pages and over 7500 words of dialogue, and was supplemented by a completed field notebook.

Again, I present my data in selected episo des or scenes, with characters and dialogue that best expose and express the complex nature and relationships within these performances, and also exploring my own 'critical space' for this task. Engaging again as a 'participant within' rather than 'foreign afar' (performative criticism), I examine the 'inside story' of events here and the different values and tensions this presentation reveals.

\subsection{Act 3, Scene 1: From here to eternity}

I met Rubi (not real name) during my first day on site here. Rubi was a current 'circuit therapy' patient attending the clinic for a daily protocol of electrical treatments, for those with a cancer diagnosis. Part of a complex programme, alongside other therapies, I understood the aim to be about cleansing and detoxifying the body. An older lady, slender and with sun-dried skin, Rubi was going to be attending daily for the next five days and she agreed to let me follow her through the 'circuit' the next morning, and participate in an interview.

The next day, I arrived early before the clinic opened to patients and joined with the staff in their morning ritual of 'smudging' with incense, and prayers for the day ahead (part of a whole programme of positive 'energy' enacted to help give their best to patients). Rubi arrived into the cosy reception/waiting area promptly at $8.30 \mathrm{am}$. Already the heat of the day outside was starting to build, making this entrance space a welcome retreat. A cool space (due to air conditioning) with tiled floors, white painted walls, a solid wooden counter, old beaten up (but loved) leather sofas, plants, icons, pictures, clocks and a stunning crystal temple, this felt to me like a really welco ming and homely area not just to arrive and wait in, but to also 'rest' in. 
Ingrid (therapy assistant, not real name) soon appeared to escort Rubi through to Room 1, where she could get changed prior to beginning her circuit. Ingrid was a pretty young woman who looked a 'relaxed professional', with a clinic (white) coat open, over a pretty sundress. Employed and trained here, Ingrid seems primarily responsible for guiding patients through this type of therapy, assisted sometimes by the student Naturopath (Holistic Practitioner's son). When Rubi emerged, she was wearing a long t-shirt with bikini briefs underneath and was shown directly into Room 3 (next door to Room 1)). She appeared 'ready for anything'.

Once inside Room 3, Rubi's circuit began. The smallest of the therapy rooms, this has the same tiled floors and white painted walls as the entire building, and is dominated by two wooden cabins, one a closed 'infrared sauna' cabin and the other an open, metal lined 'Orgone accumulator'. Rubi was due to begin on another electrical therapy machine, that was set beside the infrared cabin. For this, she reclined in a modern, yet comfortable lounger/chair within range of the electrostatic field created by the machine for 40 minutes (I conducted Rubi's interview during this time; better now than later when she may be tired). Her view from here included a simple wooden chair and nice potted plant, towards the sunlight streaming through the window. Above her head, and icon looked down on her. She seemed comfortable and relaxed throughout and we chatted freely about her health and her experiences here. When this first machine was done, Ingrid returned and moved Rubi across to the small, metal lined cabin near the window. Rubi climbed inside this and sat on a wooden stool with her feet in a large wooden bucket as Ingrid added hot 'boiling' water and an electrode to the bucket. Over the next 35 minutes, I watched with great interest as the water slowly turned from clear to a greenish-brown colour. Rubi explained to me this was the release of toxins from her system.

By now it was almost 10am and when had Ingrid returned from taking the water away, she led Rubi into Room 4, for the next stage. We crossed the hallway into Room 4, a similar sized space with a small wooden desk and chair, a white leather therapy couch to lie on and just one machine. A light and airy space, the sunlight in here illuminated a large Leonardo Da Vinci wall hanging as it shone through the window (an identical one to that I saw in a bath therapy room in Holland). Rubi climbed straight onto the couch and Ingrid placed a white tube, connected to the machine, over her chest. The machine began 'popping' as a repeat electromagnetic pulse was discharged into Rubi's system. This seemed very loud and quite 'shocking'. Done in three short spells, as the tubing gets quite hot, Ingrid returned each time to rest the 'tubing' on a fan to cool it down, before re-applying. 
After Ingrid returned the third time, we were on the move again, this time into Room 2 (just next door). Ingrid led the way and Rubi followed, settling on a wooden chair in the centre of the room, between two pieces of electrical apparatus. Rubi sat facing the window with views of nature on all sides of her (some real and some paintings) while Ingrid set the machine going. The buzz of electrostatic charge started, and this rather than the piped music became the soundtrack for the next 10 minutes. When this had finished, Ingrid came and took Rubi into Room 1 (where she first changed) for the remaining elements of her 'circuit' today. Perhaps the most 'cluttered' of the rooms, here there was a selection of machines, a therapy table in the centre and open wardrobe, where Rubi had left her clothes/shoes earlier.

Rubi removed her t-shirt now and stepped inside an ozone sauna unit. Ingrid wrapped a towel around Rubi's neck (for sweat) and closed the door so only Rubi's head was sticking out of the top. Rubi got quite chatty at this point and told me how this is more like a health club than hospital. Between the robes, the slippers and the relaxed relationships I saw, I had to agree. 15 minutes later, Rubi was now out of her sauna and lying face down on the therapy couch (uncovered, no towel) for a mixed 'manual and mechanical' massage from Ingrid, which lasted another 20 minutes. Throughout this Rubi was quiet and Ingrid appeared thoughtful and focussed (just like the saints/icons on the wall). For the last element of this session, Rubi turned to lie face up. Ingrid covered Rubi's torso and legs with a red towel and put a pillow under her head. She applied yet another electrical machine, through a 'blue gel' barrier, to Rubi's chest and neck in a methodical and unhurried manner. The piped music that had been running throughout the clinic was now audible and made the whole atmosphere feel quite restful. My time with Rubi ended after this as, once cleaned up and dressed, she went downstairs for some Osteopathy and then was due to see the Holistic Practitioner, for a consultation.

Before I explore this observation further, I want to add Rubi's narrative. This, together with the description of events above, provides the holistic perspective that I have found so important (and revealing) throughout this whole process. Her responses make for challenging reading, in terms of exposing the unseen responses of 'place' for patients and exploring definitions of CAM (is it a treatment approach or a philosophical one?).

\section{Rubi's narrative:}

I have been coming here for almost 1 year now. My daughter found this place on the internet. I have cancer, and was suffering badly from the chemo. I needed to try 
something different, and what I get here is certainly very different. I have all the therapies on offer, and I take the vitamins and supplements too. The therapies heat me, then hit me and then heal me. The Doctor [Holistic Practitioner] is very strict, but I trust him, and all the people here are friendly. They seem very happy people. They are not depressive people. They help me to feel free, and I feel looked after here, I feel at home. I find depressive patients damaging to me. In the hospital, everyone is uurgh [pulls a sad, depressive face, emphasised by miming pulling chin/jaw lower]. I would often 'escape' to be free of them. I only coped with being there through the support of my children and family. Good support can divert your attention from the bad aspects of a place, like in a hospital, but then when they leave you can feel very alone. The differences between a hospital and this centre are from "here to eternity". It is a hospital versus a hotel. There is a quiet and tranquillity here, it's a different attitude.

I had to have hair analysis, before I could begin any regimen, and I do think the most important thing here is the actual therapy, more than the place. I have never really considered the role of place in therapy, although I do think that your own 'condition' or current health can make a place feel bad or worse or better. This place is nice, it is a very calm place, and I feel calm when I am here. All of the rooms are basically the same, with just different machines in them and there is lots of nature around. The practitioners blend with the patients too, to be a part of therapy with them. I feel quite comfortable here, although I wish it were closer to my home. Cypriots do not like to travel even short distances. I always feel tired at the finish, but I enjoy the 'ritual'. I never enjoyed the 'ritual' at the hospital. That was all urban and busy, and the therapy aggressive and invasive. There was only suffering. Here there is some suffering, but also the opportunity to enjoy the process of therapy.

As a whole performance, two things here (more than any other) stood out for me. The first was the sheer contrast in the wider environment from others sites in the study and how remote this clinic felt from 'the rest of the world'. This is both an observation of geography, being literally 'in the desert' on the southern tip of Europe and located outside any identifiable urbanisation, and of relationships with/within the space as both locals and overseas patients found this place, people and provision a refuge, to escape the world and its problems. 

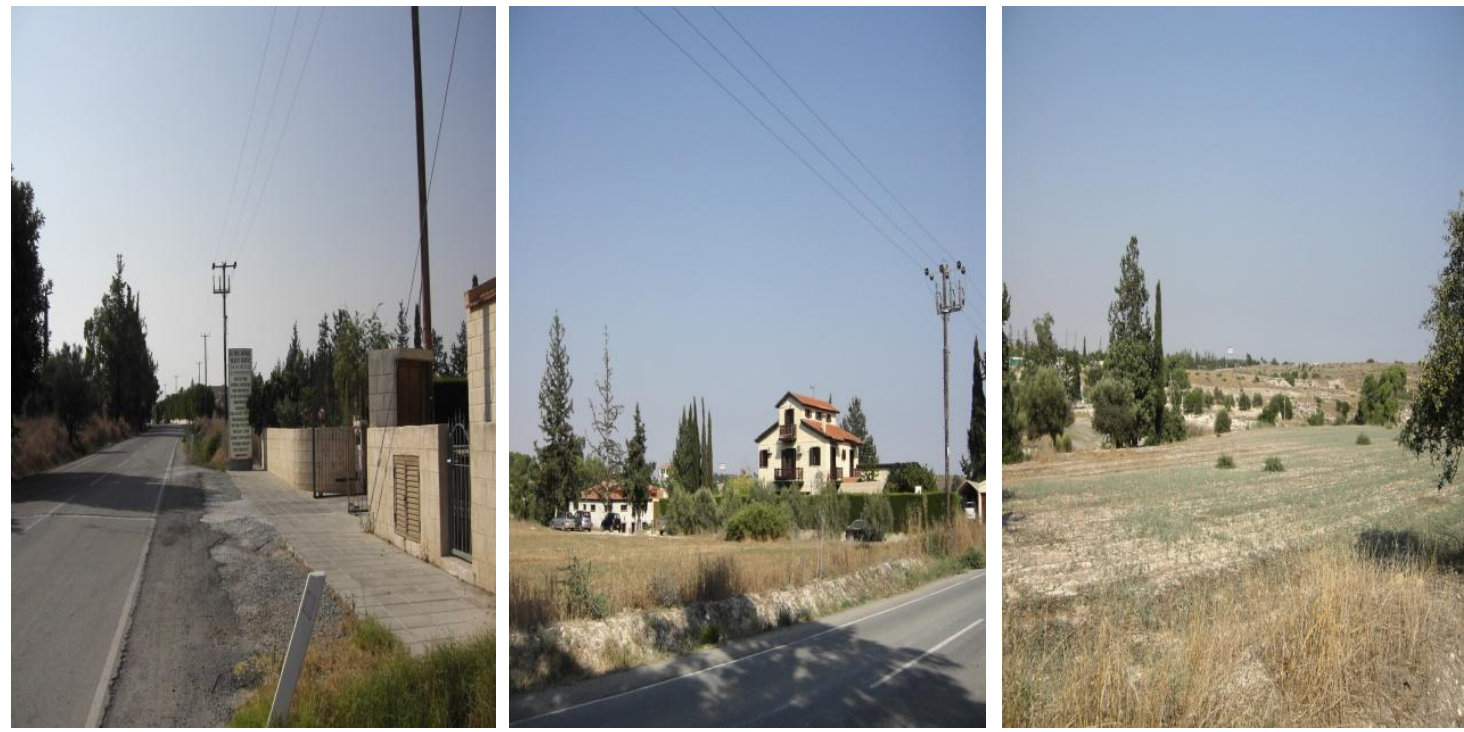

Triptych 8.1: The clinic exterior and local landscape

A 'protected environment' then, surrounded by nature, I found this place offered beauty, peace, space and light, the like of which I did not encounter in either UK or Holland. Not completely cut off from civilisation, but with no immediate neighbours visible beyond farming land and the occasional properties dotted into the hillside, this place felt isolated, away from prying eyes which I found made it very easy to 'disconnect' from the harsh realities of life and leave behind any troubles associated with this, at least for a little while (and not only because the internet coverage was so patchy).

Rubi expressed it well herself, in that "the differences between a hospital and this centre are from here to eternity". This was something I had felt from the first mo ment I arrived on site. I had pulled into the dusty unmade car park, stepped out of the car and took in my surroundings. It had taken me only a short drive along the fairly quiet motorway to reach the Centre. As I stood, taking in the vista, I understood Rubi's sense of eternity, of 'getting away from it all', even if my reasons for attending were different (in physicality if not philosophy).

Engaging in the ideas and experiences that are offered here are, for many, so far removed from their everyday lives, that even just an hour of escape can feel 'like a holiday' - a holiday from whatever pain, misery, anguish or despair that life creates and mainstream practice fails to adequately address. The patients who choose to come here, literally 'leave the world behind' and create a 'space' to rediscover themselves and to heal.

I identified with this, and the power of such an act, as throughout this research process I too have been 'escaping the real world' and seeking a space where it was permitted, even advantageous, to rediscover my 'centre' (who I am and what I really believe in) and to heal. I 
did this primarily by attending numerous CAM research conferences, in the UK and overseas hoping to find inspiration, kindred spirits and a 'safe space' to explore holistic solutions to my life's frustrations. I knew from prior experiences (of therapy and study) that 'real CAMs' are much more colourful and more healing than practical, sanitary, fast and reductionist deliveries suggest and hoped these environments would provide a 'new home' to explore new ideas. This often seemed to work too, as I connected with a number of different characters, also seeking salutogenesis in their professional (and life) journeys. This was also 'tempered' by often esteemed characters (many of whom I had 'read' as a student) who seemed 'stuck' in the default medical model of research, with no easy way out. This was disappointing, even heartbreaking, for me as what I learned from their performances was that 'ideas' are fine, but they don't 'fit' our reality. I couldn't accept this and vowed to keep looking to find a place to rest (my ideas). This was, after all, not just a professional journey, but a life's quest. With invaluable input, contacts and support however, I generally found these encounters supportive and, in that sense, healing ones.
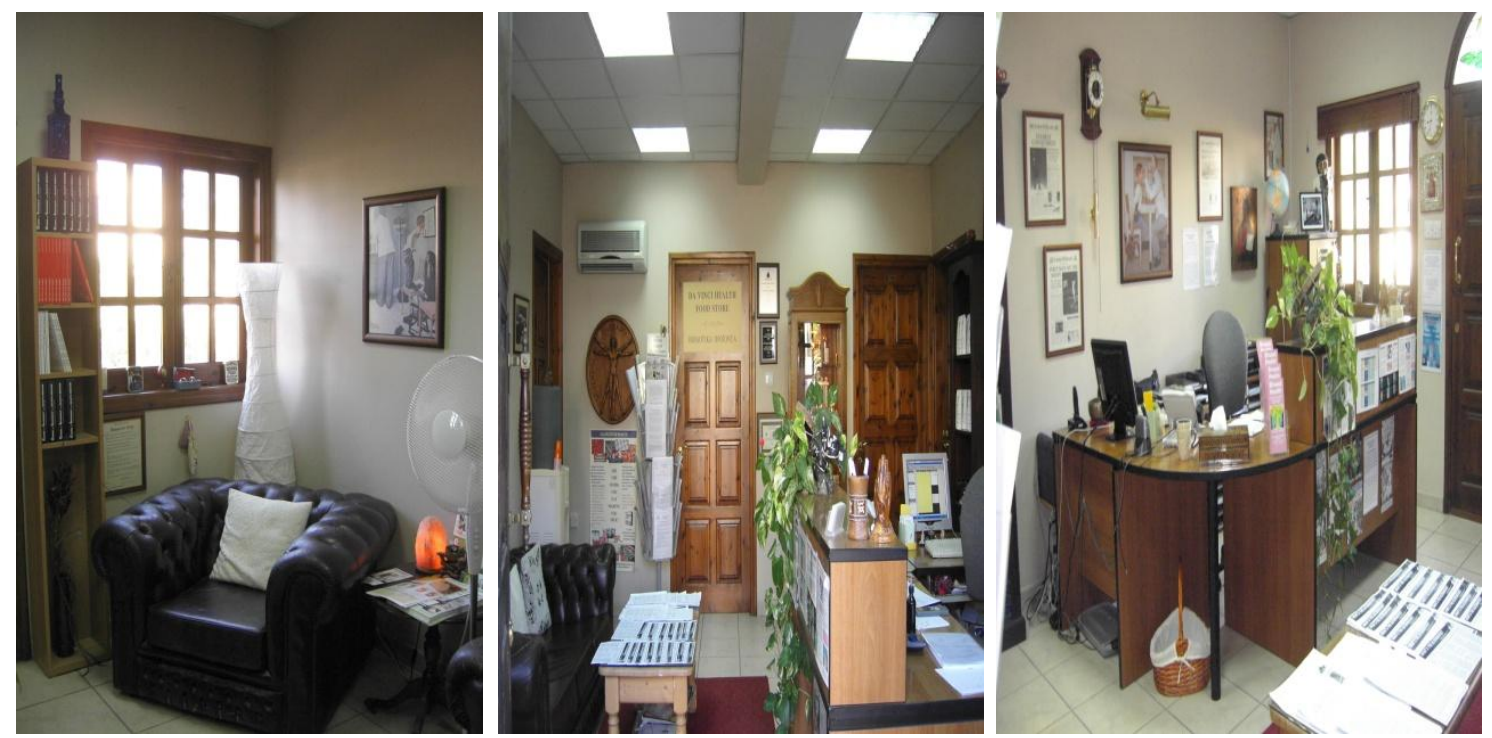

Triptych 8.2: The shared waiting and reception area

Patients come to this Centre to also find a 'safe space', to escape, be 'themselves' and find healing. A setting that is aesthetic not simply practical, with natural/quality materials instead of cheap/sanitary ones, offering time over hurry and complex rather than minimalist approaches with even an 'over display' of spirituality and secular faith, this felt supportive not only of ideas but of actions, in a similar way to the settings in Holland did. Within this vista and the philosophies of these characters, I became further convinced that anything can be possible, where the 'space' allows. 
For Rubi, having spent many years within conventional medical care, oncology hospitals and under chemotherapy, this place was equally an escape from the real world and from the clinical world of sickness, death and misery that she has come to associate with this. This was like an exaggerated version of the observations by Nora (Chapter 7) about having therapy where there were not so many sick people. In fact, it was hard, from watching and spending time with Rubi, to imagine she probably shouldn't even be here/alive (according to her oncologists). Here, she was pursuing health (salutogenesis) and although her body had been through some incredible trauma, the light in her eyes said to me she wasn't done yet!
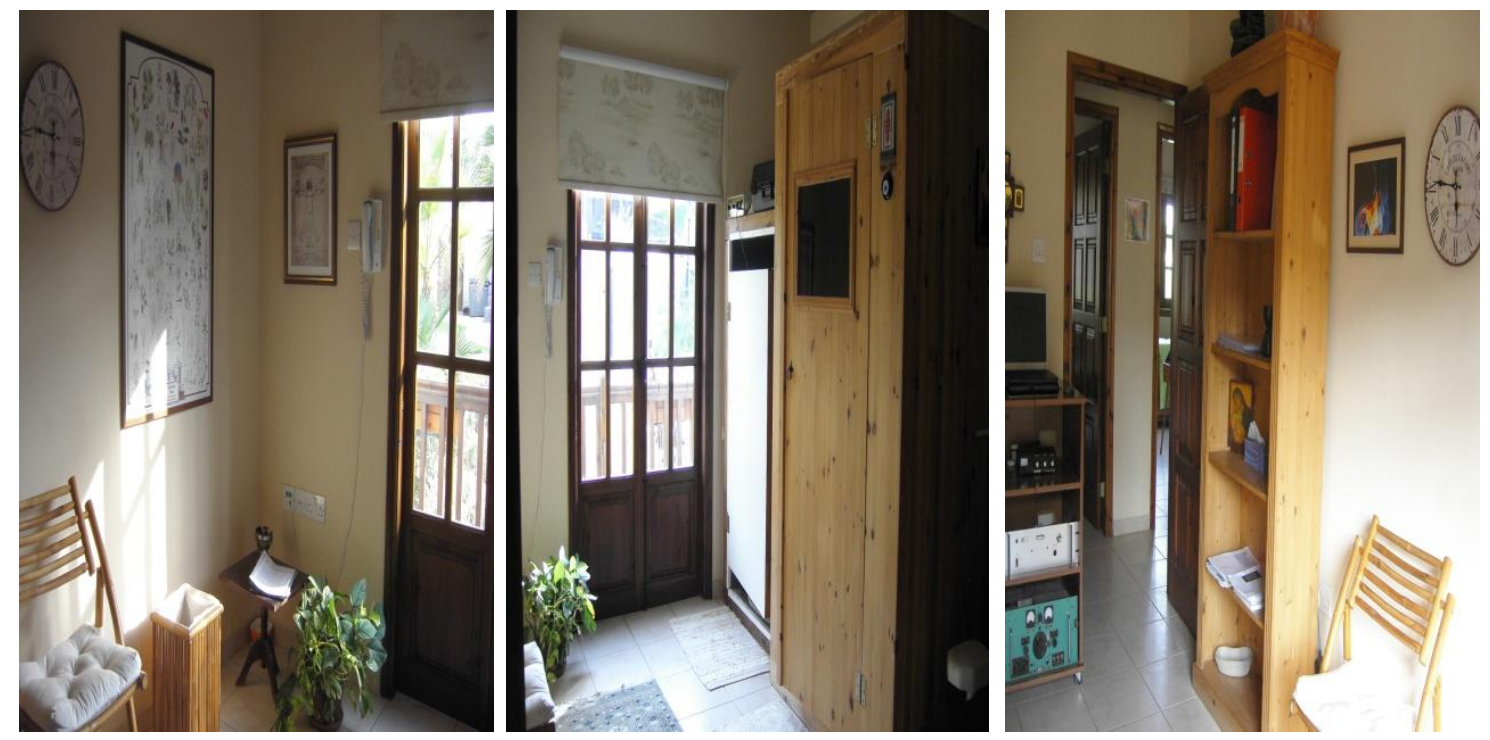

Triptych 8.3: Therapy room 3

Being free from the prying eyes of mainstream society may be a vital factor in allowing this place, these practitioners and the patients who come here to pursue an alternative approach (much like conferences are held away from academic settings of Universities, in usually 'nice' hotels). Something I have certainly found in my own life is that it can be difficult to exercise the freedom to heal when those around me are critical regarding the ways I choose to do this (for many years I have always sought CAMs as my primary care, with conventional medicine a last resort). Here, removed from an urban or City location, all have more freedom (more space) to act authentically and unconventionally without fear, and this is how patients appeared to enter these. This situation made a distinct contrast to the UK site, where patients and practitioners operate in full view of the mainstream and feel compelled, almost, to look conventional (less conflict).

The second issue that I want to explore further is the way that the attitude of delivery affects the ways patients relate to, and benefit from, the interventions here. In any other 
environment I feel some of the interventions I saw here would be regarded as more medical than natural therapies. In particular, there is a heavy reliance on electrical therapy machines and technical diagnostics, similar to those seen in mainstream medicine. Delivered under a holistic ethos, however, these seem to take on a different potential and with seemingly amazing results.

This was the case for Rubi. Like everyone who comes here, she started with a comprehensive diagnosis before being prescribed a customised plan of diet, therapies, counselling and lifestyle changes to cleanse and detoxify her whole body and spirit. Delivered under a "different attitude" than she has experienced before, this strict approach was viewed as a total contrast in place and practice to the sterile, soulless factories [hospitals] where the people never smile and there is little hope.
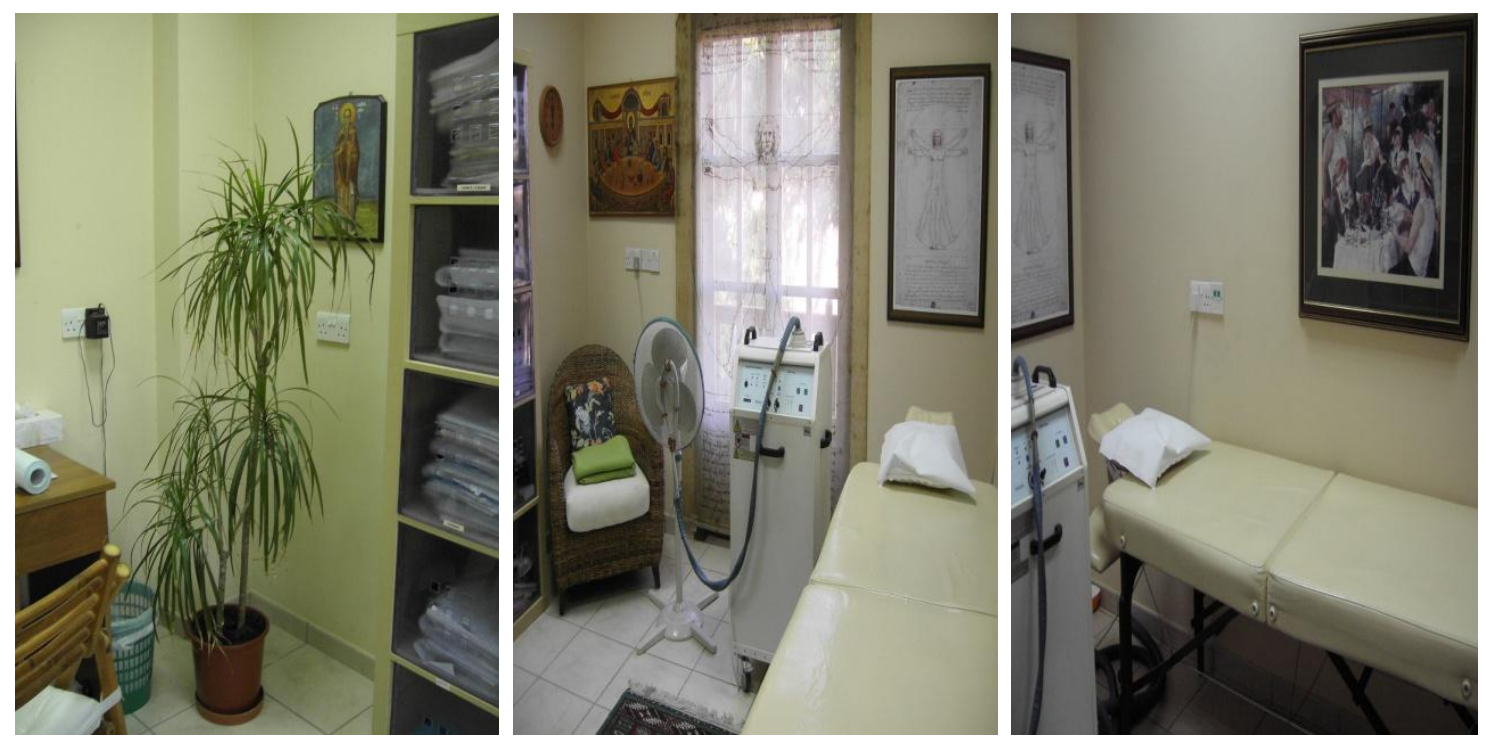

Triptych 8.4: Therapy room 4

An attitude to life and way of understanding the causes of health and illness that is different from mainstream medical thinking, what Rubi talks of is embedded in the philosophies of practitioners, which they express through the ways they care for and stage these surroundings. It is the reason they partake in various rituals, care for themselves and care for others in a multi-level approach (the Holistic Practitioner identified these 'levels' for me as physical, psycho-emotional, spiritual, energetic, environmental and social).

Here, thera pies are not applied to treat conditions directly, but to treat people with conditions. This is both a legal and paradigmatical shift, common among many CAMs yet almost totally unrecognised within CAM trials. For patients, this manifests as a whole approach in which they (not their dis-ease) are in the centre, thus it will be they who improve, not simply their 
symptoms or disease. Certainly, it may be hard to argue that bowls of salt in every room have an effect on disease or that 'icons' on the walls and incense rituals could provide cures and neither is this the intention. Such items instead suggest a more dynamic, unseen effect that supports their being there. Rather than directly affecting problems, these aim to affect the conditions of the environment and, through this, affect peoples' relationships here with therapy, with thera pists and with themselves.
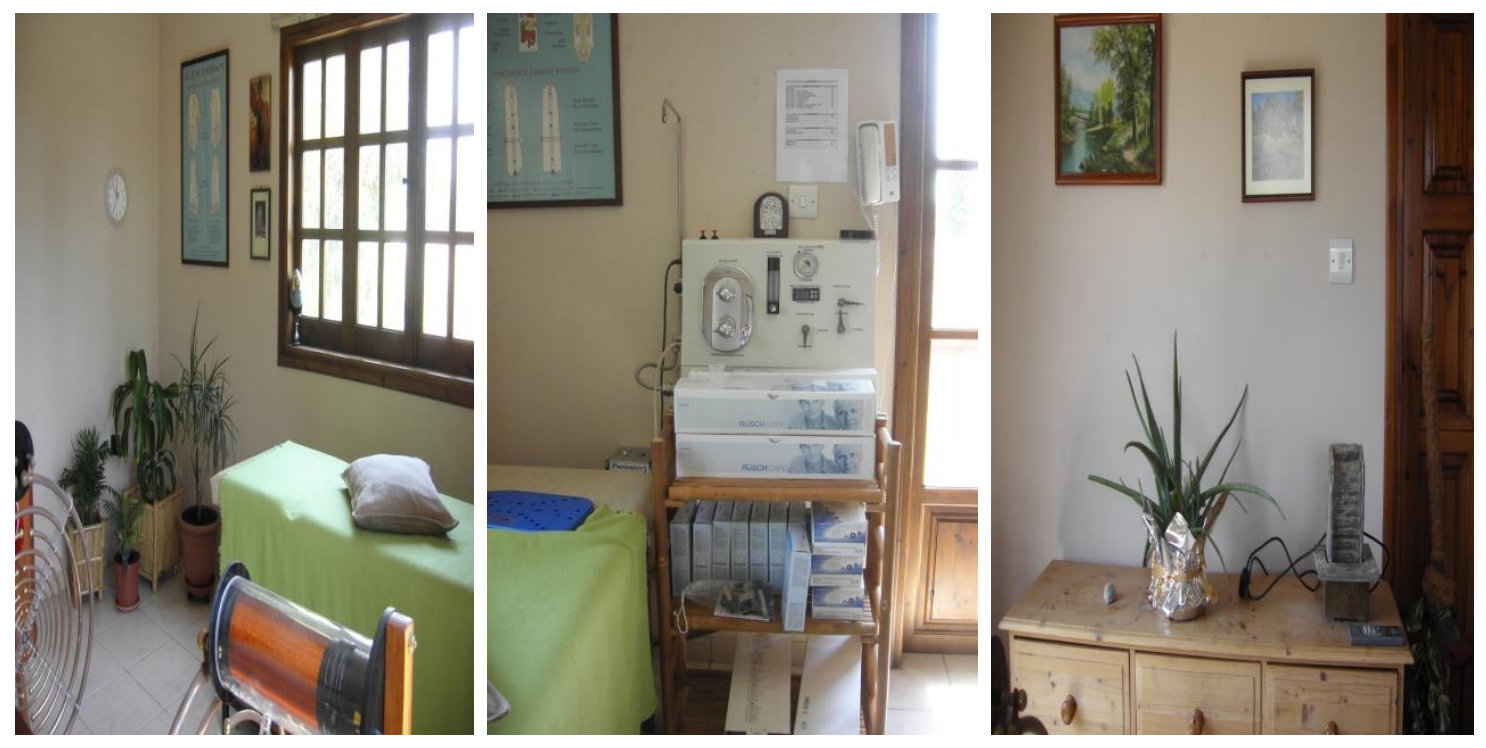

Triptych 8.5: Therapy room 2

Set against this backdrop (a backdrop of wider ideas and potential outcomes), the clinical white coats worn by practitioners and the array of electrical diagnostic and therapy machines seem less indicative of mainstream/medical approaches than they would in a hospital style place. This 'space', therefore, carries a different potential than patients have come to experience and expect from therapy. This brings me back to something Rubi said about her time in the hospital. There, she said "everyone is uurgh" and she pulled a long face. The nature of that environment, as Rubi described it, seemed to have a depressive effect on her (and potentially other people also, patients and staff) which Rubi felt was so damaging she would often try to escape, either physically or into the company of others. Here, in extreme contrast, she (and I) found everyone to be "happy people" which, almost irrespective of the treatments delivered, created different potential for the staff, the patients and for therapy.

While providing an escape from the real world of harsh realities, oppressive (and often unsuccessful) healthcare regimens and a lack of hope for healing, characters who comes here are often escaping also into this environment, which suggested to me there must be something healing or beneficial about this place, the people and the narratives they dramatise 
in their attitudes and acts (beyond any specific interventions). Several patients, for example, spoke of how peaceful and restorative this natural, rural setting was or how, through the ways different therapies are combined here, they personally "have died and was resurrected", their "whole self has changed" or they were "born again". One patient even suggested the Holistic Practitioner "is a God, because he creates miracles" (direct excerpts from patient interviews).

Amid these powerful accounts, I found it increasingly difficult to dismiss the subtle approaches and effects these practitioners include (with purpose) within this whole production as peripheral or non-specific effects (even placebos). But then I was here to expose and explore connections and relationships between these characters, plot and setting and here I found plenty to work with. Indeed, I had never seen anything quite like the presentation of performances I saw here and it all seemed to work!

\subsection{Act 3, Scene 2: Me, myself and I}

All of this comes from the Holistic Practitioner through into his staff and the space he has created to support him and his work. A complex and learned character, I found him a fascinating person to watch, listen and talk to. He is enigmatic in a very 'guru-like' way, and so convinced of his own knowledge, experiences and the results he has clinically demonstrated through patient outcomes, that it is hard to argue with him.

Theo (not real name) is truly the power behind this throne, his dominance clear from the purposeful way he strides across the room and the language others use to talk about/to him (the Doctor). My interview with Theo, in reality, revealed very little that I had not gleaned from absorbing the environment he has created to dramatise and support his therapeutic model (and worldview), but did provide substance to the man and his acts that I share now so that it may explored further.

\section{Theo's narrative:}

I was born in the UK, to Cypriot parents. I moved to Cyprus [came home] when I was 27 and set up a practice in psychology and clinical sexology. But then I became ill, with glandular fever, which left me with symptoms like Chronic Fatigue Syndrome. I was the first sexologist on the Island, and had a very busy practice which I could not keep up. I tired easy and could only work for a few hours a day. After spending 7 years visiting regular Doctors, I was no better, and had also developed systemic candidiasis ['leaky' gut]). All the antibiotics and antihistamines had poisoned me. I began to look at natural medicine. I did a lot of reading, which suggested 'other ways'. So I returned to 
England, and studied nutrition with one of the authors I had been reading. I then studied naturopathy, iridology, homeopathy, su jok acupuncture and homotoxicology. Each had benefits. After 11 years, 10 degrees and 2 doctorates, I had cured myself. I began to put together the protocols I use with patients today as a direct result of my own healing journey. Diagnosis is the key. I do this in addition to treatments using the IDEL diagnostic system that I developed. It creates a roadmap of patient pathogenesis and guides my work. I have a lot of equipment. I don't believe you can just treat with supplements.

I find that combining methods is more effective than single modality care. This is what I practise with my patients and what I teach to students through the College here. Practitioners need to know about all modalities in order to fully help people, but Universities only teach single modality models. Many of my patients have zero prognosis metastatic cancers and I am getting great results with them, using a multimodality approach. Defying 'zero prognosis' gives me spiritual reward and affirms faith in my methods and treatment approach. It is my goal to become more and more successful at treating people, with metastatic cancers. Energy medicine is an additional modality that I hold great interest in. I just ordered a new Bio-resonance machine because of a paper I read. I have also set up a charity to fund therapies here, for those who cannot pay. This is where profits from the [on site] health store go. Giving is a way of life for me now. I believe a wider justice exists in the cosmos, where genuine care, concern and service earns the appreciation of positive energies that will protect me and my work from any negative ones. I have many 'active enemies', both regular Doctors and the Cyprus Medical Association. But I am not afraid of them. I am committed to practice and my patients. I will never retire. There is so much to do, both clinically and in research. I have many research ideas.

Balance, in the external environment, is very important for therapy work. It grounds the practitioner. When I first came to Cyprus, my practice was in the City, in the same apartment I lived. It was noisy and polluted, but better than London, where I had worked previously, and much less stress. Then I moved to a separate apartment, before moving to this house, in the country. I put the apartments together and commuted there to work. Then the infrastructure reached here, so I built a wing on my house and moved the practice. I built the current clinic, in my garden, when I needed more space. I used dowsing rods in the design and construction of the centre, to find 
where the geopathic stress lines are (both positive and negative). I have also traced a Ley line to the side of the clinic that runs through 5 churches over a $5 / 6 \mathrm{~km}$ distance. This is a real power source. We have 7 conifers all growing in the same area, because of it. A natural environment is best for natural therapy, and I also use tachyon energy to help maintain balance. The energy of this setting is more important than its' physical qualities, although both are part of therapy, as am I. I need to be healthy to help my patients. There are some items here that to some people would seem superfluous to healing per se, but they are part of the whole energetic balance. I especially get comfort from the sacred icons. I have a Virgin Mary from 1690. I pay reverence to these, and they inspire me. I would not work in an unbalanced place. A negative energy space results in energetic imbalance of the practitioner and this passes to patients in therapy work. Money motivations can do this too. Even if people are not sensitive to energies, and not all people are, that does not stop them from 'feeling'.

I am a perfectionist and always look for new ways to enhance patient care. I used to work with other practitioners, but find that my broader education means I think faster than they do. Their limited views can make them a burden to this type of practice. I would not like to expand further. It would only be burdensome for patients, as well as costly and impractical. We would have to have staff meetings, which would leave less time for patients. I like the small team I have now. They each provide something unique. As an integrated team we provide a holistic service. My staff are sensitive characters and we work as a family. They are rare jewels with energetically open hearts, and are caring. Caring motivation is healing in itself, and the relationships we have here are integral to the practice. They allow me to focus on my clinical work.

Theo's narrative demonstrates that he is the core of this practice. His own illness and healing journey are the foundations for his self-belief, and his commitment to helping others whom conventional medicine has failed are built upon this. This is a similar sentiment to that expressed by Simon in Chapter 6 (UK site) but where Simon felt unable to act fully upon his inner beliefs, Theo has no such inhibitions. I observed this distinction initially as a difference in cultural or economic environments, whereby Simon felt he needed to 'look mainstream' in order to sustain the financial viability of his practice and Theo (owning rather than renting his premises) did not. However, when Theo shared with me privately that he would even be willing to go to prison to defend this model if he had to (he maintains it's a great place to read 
and do research!) I realised that his conviction went far beyond Simon's illuminating every aspect of his being with an inner confidence that lights up this whole place.

The distinctions between Simon and Theo, however, are not only philosophical, but also a direct response to their own, unique contexts. Simon's healing journey was limited to single modality care (reflecting the nature of his ailments) and this is what he sought and now delivers. He makes space within his Centre for others too, but they do not influence his thinking or clinical approach. Theo's healing journey and his resulting practice, due to the more complex nature of his situation, took in many modalities which has, in turn, influenced his practice experiences, training and thinking.
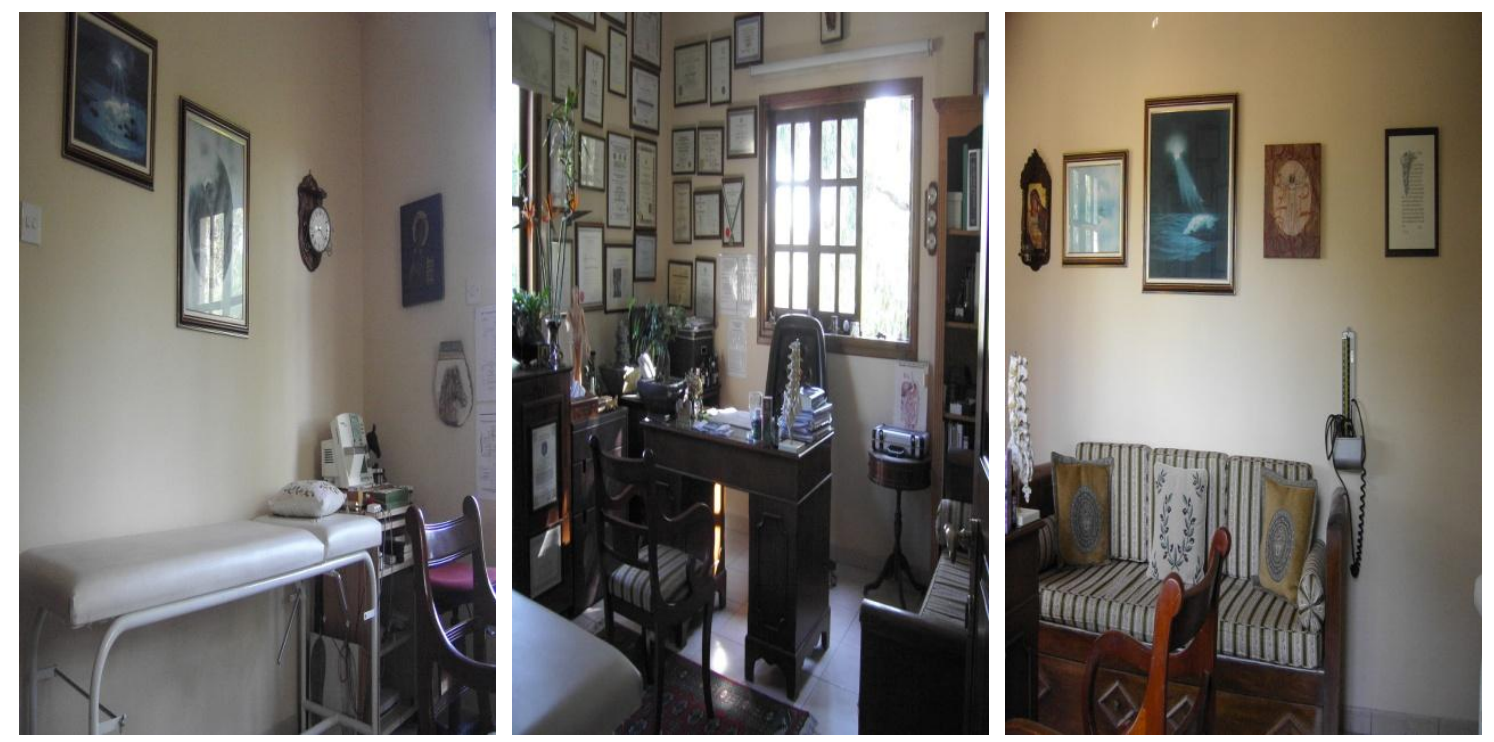

Triptych 8.6: Main consultation office

Also following different paths in terms of their 'continuing professional development' (CPD is a very hot to pic within CAM for ensuring practice standards) Simon and Theo have each come to adopt different philosophies, patient demographics and clinical methods. From my position, as a CAM practitioner, I recognise the difference between both of these in relation to my position, which is somewhere in the middle. Like Simon, I have trained in a single CAM modality as a response to physical health issues. However, I also like Theo, recognised this created artificial limits to recovery and so studied multiple CAM philosophies to enhance my knowledge and applications. In Chapter 6, I identified the ways that my approach is different to Simon's and now I want use that informed position to explore my relationship with Theo.

I observed Theo's model of care to be based on blending many different theories and modalities. Achieving coherence in such an act can be a real challenge, and something that I can relate to as my therapy is based on a 'physical' practice supported by biomedical theory, 
yet I deliver it within a dynamic and holistic ethos. Like Theo, I find that visible expressions of 'integration' can aid patients to leap with me between world(view)s and connect with themselves and my therapy in a new and enabling way. This is why I stage my environments equally with practical items (therapy table, towels, oils etc), semiotic props (candles, books, fresh flowers etc) and esoteric elements (tachyon crystals, angel images etc) to permit this. I believe this opens the potential for them to gain more than physical relief, even if this is all they came for, and provides me the potential to offer support and opportunities beyond physical relief (through conversation and education) leading to better wholeness/wellness, for us all. Made easier by autonomy (unlike Simon who shares this responsibility with other practitioners) I do confine my expressions to those I feel I can gain acceptance for (different than those that are acceptable).

Theo goes further than this, having both autonomy and the unlimited support of those around him to express and enact his therapy with a conviction and commitment, similar to the practitioners in Holland (where everyone I met seemed to share the same holistic vision). He therefore appears not to be inhibited by anything except the idea of a 'professional' image, which is clearly manifest in the white clinic tunic he wears (as do others) and his proud display of qualifications/certificates. This provides his 'link' to credibility and acceptability as he steers his patients through what is often 'uncharted territory' for them. Whether this is more important for him than it is for them, I am unsure. Perhaps the two are inextricably linked.

Anchored by this, Theo then seems to indulge every whim or argument he can find that originates from or fits within his overarching philosophy. Examples of this are evident throughout the clinic in his choices of artwork, selected icons and collections of antiques, clocks and model cars. Some of these 'props' speak of him (his interests, his beliefs) while others speak for him (his philosophies, his intentions), together creating a dialogue that patients join in, supporting their active engagement in his complex prescriptions (therapies, diet, supplements etc). Although, it is notable that this dialogue is largely one in which he speaks and everyone else listens.

It is easy to say that this is due to his status (senior practitioner and owner of the Centre) or higher knowledge, experience and training than anyone else here, but I felt this was only part of the story. He surrounds himself with 'angels' selected for their open hearts more than any clinical qualifications, finding that mere 'mortals' only slow him down ("I used to work with other practitioners...their limited views can make them a burden to this type of practice"). More critically, I found Theo was able to be at the centre of things because the other people 
(patients and staff) and this place give him the 'space' to do this. Like a 'guru' his doctrines are compelling and his results indisputable (as I said earlier, many of the patients I spoke to shouldn't have 'been here' according to their oncologists) which means those around him, myself included for a time, become almost spellbound in his presence and willing followers. Patient comments (as noted earlier) like feeling to "have died and was resurrected" or "he creates miracles" (direct patient excerpts) further elevate Theo's status and power. This 'space' is not somewhere he applies therapy but somewhere he is therapy.

Together with this willing and hopeful audience, this unique location, style of practice and practitioner can equally, if differently be considered responsible for the "different" attitudes and outcomes that patients (and I) experienced here. More than at any Centre in this whole study, the integral role of the practitioner is elevated to a place where it cannot be ignored. Even though many of the specific modalities are applied by assistants (rather than Theo himself) he is always there in the prescription, in the place and in the outcomes.
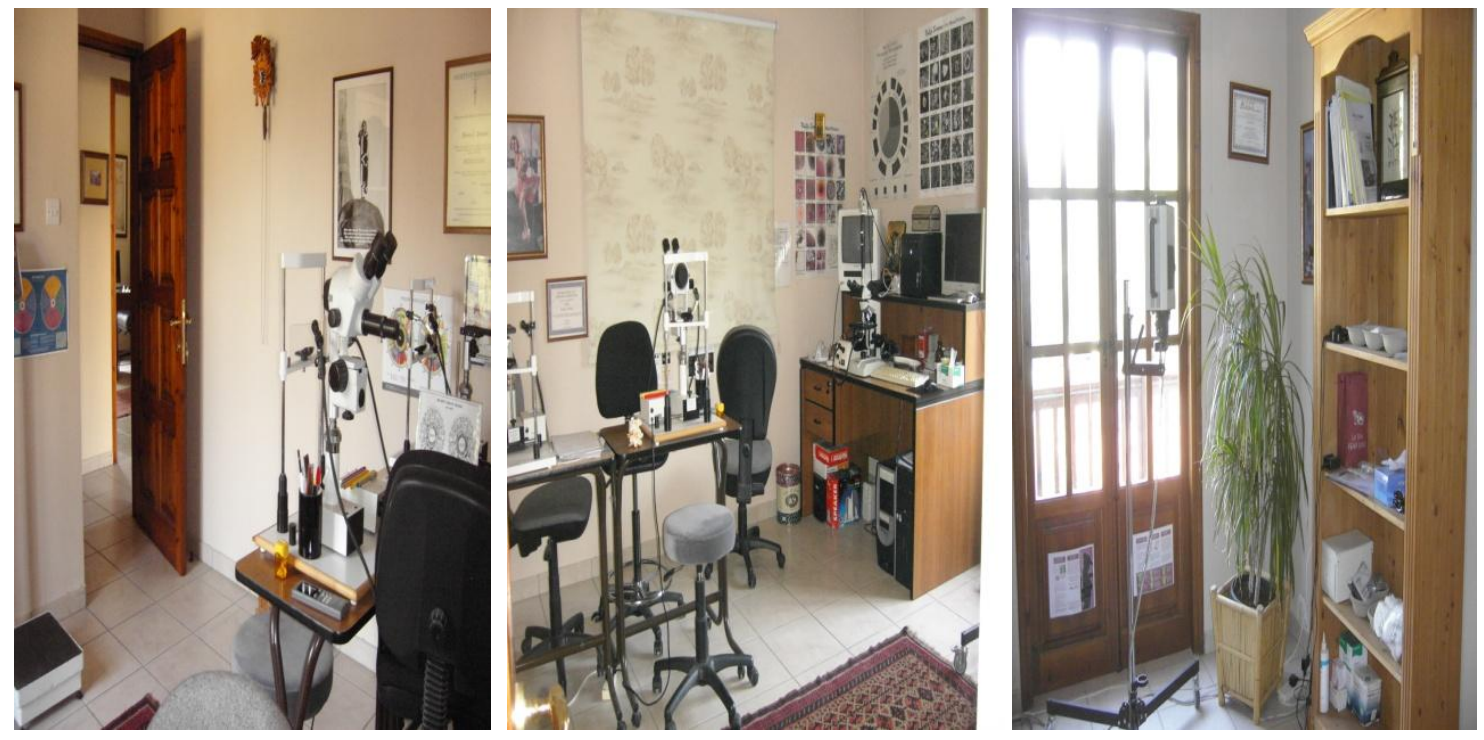

Triptych 8.7: The diagnostic suite

Theo also had a direct impact in my research as he insisted on controlling whom I had access to, to enrol in the study. This does not mean he recruited for me, but that he only put forward people he would be happy for me to recruit (those he felt were 'stable' enough in their recovery to withstand any disturbance participation may result in) as if participation in my research could only be harmful to their health? This was different to the controlled access in UK, where practitioners suggested those who may best be able to 'spare the time' or make 'good candidates' and a complete contrast to Holland where anyone was free to participate if they chose. 
Keen also to ensure I did not adversely disrupt the energies within the clinic, by my presence, Theo sought to manage any disturbance by regular review. Having become aware of the sensitive nature of his 'work', I understood these concerns and always sought to blend in to this environment. However, there came a point when Theo preferred me to spend less time on site, unless I had pre-arranged to interview or observe someone. Theo's lack of compromise in this situation was matched equally by the lack of compromise expressed in his whole approach. I had no choice but to accept this, although I found this tough to take as I felt he was questioning my integrity and intent. Like the child who has fallen out of favour with the parent, I was upset and angry. Why didn't Theo like having me around anymore? What had I done to 'disturb' the balance? Was it something I could fix? I had, or at least I thought, always been consistently polite and considerate of his work. Theo had already said that he needed to be healthy to help his patients so maybe the issue was actually that my presence was starting to affect him (or his team) adversely. Either way, I eventually began to get the sense that the entire clinic was waiting to exhale. This again is a unique point, as while the UK site also reached a limit for my intrusion, this took longer there and I spent more time on site. Holland, in some contrast, would have kept me for longer if they could.

This suggests a new perspective for the phenomenon of 'the researcher effect' and new concerns regarding quality versus quantity, in real world research. This is something I briefly discussed in my literature review, about how the qualities of the researcher can influence the quality of the data achieved. My experience here suggests that the character of the researcher and the relationships they build within real-world 'spaces' with other characters can also determine the quantity of data achieved. Here I was seeking quality in quantity with an emphasis on the former, and my relationship with Theo (and others) for whatever reason, seemed to limit this. His controlling interest meant I had no opportunity to soothe this, which contains a lesson for me for the future.

\subsection{Act 3, Scene 3: The accidental therapist}

Another 'outsider', but one with more clear role and benefit, is Henry (not real name). Henry provides Osteopathy here as an external therapist and comes into the clinic by request, if Theo feels he wants a patient to add this approach.

Henry's journey into therapy is also a complex one, which he shared in his interview with me. Throughout his story, it emerges that his feeling and aims for therapy have always been shaped or modified by the space (including people) where he delivers it. This is not necessarily a conscious, restrictive measure on Henry's part, but more a response to the context and the 
limits this imposes on the potential of his 'art'. Here, under the example set by Theo, he is more free to practise in congruence with the original philosophy of this method than at any time since qualifying. This suits him now (at this stage in his life).

Henry's narrative:

I'm what you may call an accidental practitioner, really. Certainly how I came to be living and working in Cyprus is all a series of coincidences. I was originally a Captain in the British Army, but after a number of bad postings, I got fed up with the job and resigned my commission. So I needed to find another job, but I didn't want to work in HR or Personnel or anything like that. Then, by chance, I met an old family friend who happened to be an Osteopath. He had treated both my parents and even my grandmother. HIs work sounded interesting and he gave me the name of the Dean of a College in the UK, where I could train. I got an interview and thought "this is it for me". I took a 4 year training course in Osteopathy and Naturopathy and straight away, enjoyed the work. It wasn't long before I became involved in professional bodies, lobbying parliament etc. But after a while I got fed up with the politics, so reverted back to just being a practitioner. Back then you couldn't just start a practice, you had to join an established one, so I joined a practice in West-End London initially to help out a friend who was sick but then he died and I was left running the practice. But then I had my family and the local schools were terrible and we couldn't afford the private school fees, so we moved to East Kent where we stayed for the next 25 years. It was after selling up there that I moved to New Zealand. That was very different. They have state recognition there, and many people had insurance to pay for their treatment. So I worked in both employed and self employed practices in a variety of places and also did some teaching, until I got fed up with academic politics. Eventually we wanted to come back to Europe, but by then the UK was too fast, too aggressive and too expensive. So here I am, in Cyprus.

I already knew of this clinic, and practitioner, before I moved to Cyprus. It just happened, when I turned up, that he was short of someone to help him out. I used to help with the circuit therapy, but now I just do the Osteopathy. I'm quite happy with that. I come out here a couple times a week and I like the lack of responsibility. It frees me to see more patients and I can take my time with them. I relate well to the approach within this clinic. Now I am older, I seem to have become more interested in the Naturopathic side of work, rather than just the quick fix of Osteopathy. I've 
recently rediscovered the original value and skills of my training and I also look for energy causes. Osteopathy can be a gateway or a bridge between mainstream and other therapy approaches. I generally find people are often more open to other suggestions after they have first experienced some physical improvement. Osteopathy is good, but it's not always enough for people. I also have a small practice, in my own home, but I'm not allowed to advertise, because of the local Doctors Union. There is a lot of professional jealousy here [in Cyprus].

There are clear differences between this setting and regular settings. I think this clinic is very appropriate for my work. The environment is all important and there is a good, general atmosphere. It is a peaceful and natural landscape, and the energy work is done well. It is very different to other places I have worked. Patient expectations impact performance attitudes and approach, which as a therapist you often try to fit. In the UK, it was all sophisticated and wealthy, it didn't suit plinky plonky sounds. In New Zealand, the pace was very busy, and the style of practise was to give shorter treatments, to get the next one through. That was 'less good'for patients. Here, there is a more relaxed approach, with music, feng shui, fresh air, trees and trickling water from the pool. There are also lots of icons, mainly for the Cypriots. Many would worry about going into a room without any.

Things happen in bursts here, and all of this downstairs [development] is a fairly recent addition. I used to do my work upstairs, moving from room to room. This space is much better and I feel more settled now, but at the same time I have become disconnected from [receptionist] and the reception area. Currently, $90 \%$ of the clinical work happens upstairs, and so I am a little isolated down here. This may improve when the computer and telephone are installed. My room is not personal to me, but I quite like it, and have no desire to change it. The clinic is vision of a single person, and so there is continuity through it. Patients appear notably and visibly more relaxed here, and I feel reasonably relaxed here too. They settle down more quickly and feel more inclined to communicate. This makes adjustments easier - unless they just had Papimi [a therapy], which has the opposite effect. There is also a defibrillator on site, so they can deal with minor emergencies. I think this gives an added value of confidence.

Delivering what seems to be the only therapy here that Theo is not trained or qualified to provide himself, Henry works with some autonomy but is also mindful that he is here 'by invitation' only. His former occupation (Captain in the British Army) means Henry doesn't 
mind 'taking orders' from Theo and this seems to ensure a smooth and workable relationship (better than the one I enjoyed with him, but then I don't like taking orders).

This aside, I identify on many levels with Henry's story. As a practitioner myself, I have absorbed the impact of different settings and the model they apply/expect me to deliver - and complied with that. This was in my earlier days, when I knew more about my therapy than I did about myself. For Henry, his initial enthusiasm for the art of Osteopathy has taken him into the worlds of political lobbying, busy 'quick fix' practice and academic teaching. There, he has found it easy to get caught up in the prevailing culture, even though modifying his practice may be less beneficial for him and his patients. It is difficult to see, until you have both been in and stepped out of these environments, that different cultures each leave their mark in term of restricting or enabling potential, both now and going forwards.

I have experienced this also, so can identify with Henry when he talks about how, now that he was drawn to more Naturopathic and energy causes, this place is very appropriate for his work, and that being more aware of these he recognises the limitations of Osteopathy as a treatment and its potential as a gateway into other approaches (particularly for patients who may not otherwise consider them). This is the same transformative perspective I have developed to maintain my own clinical practice, as discussed in the previous 'scene'. Indeed, from this perspective, Henry's model of practice here may have more potential than it has had in all his clinical history.
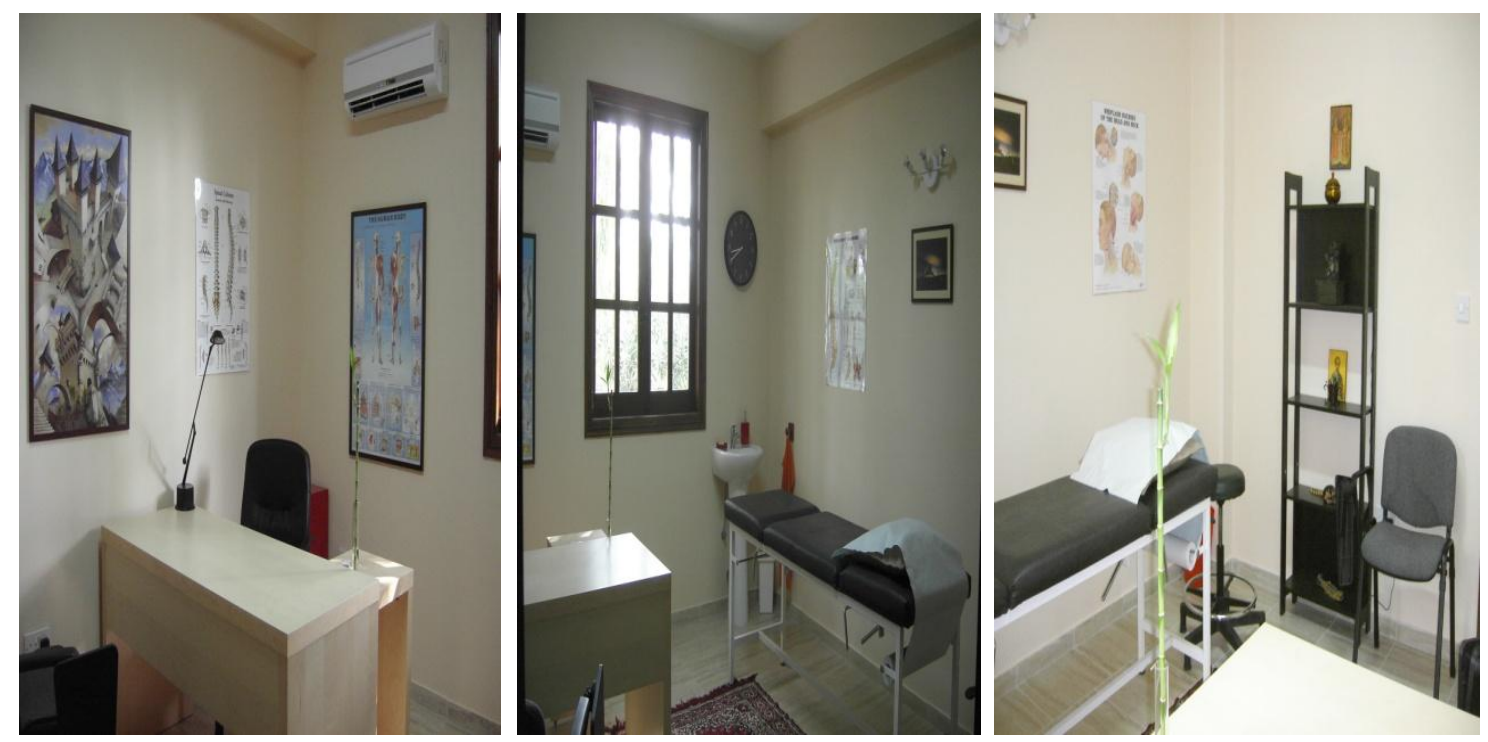

Triptych 8.8: Osteopathy room

Similarly, in research, I have also absorbed the influence of academia, including the models and expectations of different researchers, professors and my supervisors. At times I have, 
again, complied which has either been to my benefit or my cost. At other times, I have refused or refuted their suggestions (much like Theo refutes zero-prognoses), again to either my benefit or my cost. This has caused me to perform somersaults as I tried to untangle and express my aims and ideologies, often feeling I was under attack from those who were either unable or unwilling to engage with what I had to say. Indeed, it was both refreshing and disheartening when, in conferences, I have found those with the most to say being the least listened to (refreshing because I was not alone in this and disheartening for the same reason!).

Coming to know myself just as Henry has done, has been a critical component and I would suggest that any practitioner or researcher who does not know themselves (and of course I thought I did until I tested what I thought!) cannot know if they are truly limiting or creating potential in their work. I entered this site with a holistic view that I felt would enable me to engage with these performances in a multi-dimensional way and I have, but I also found that my connections both with characters and their data could only have the potential I gave it. As I have grown through this process I have learned to connect much more to myself and this has enabled me to develop further and deeper connections with my data. Much of this has been uncomfortable, but the rewards have been a mazing and revealing.

As part of this, Henry has been like a mirror, challenging me to consider what I am drawn to, where I feel is an appropriate place for my work and whether I need to change anything about this. This brings me back to an issue that Theo raised in regard to my spending more time on site than he felt was required. At the time I felt like I needed to be present in order to capture what everyone was doing/saying. Certainly, within theatrical performances, much of the story can be gleaned from what is done separately from what is said. Also, this study is part of my researcher training; it is not only about producing research findings but about producing an independent researcher (me) at the end of it. I am not sure that Theo understood this and maybe I assumed he would, because he has completed his own (two actually) doctorate degrees. At times, when I was locked in the excitement of my research and excited by my findings, I forgot this too and this shows both my arrogance and naivety (which I hope to have moved on, and learned from now).

But maybe also, as some level like Henry, I too find that applying a method no-one else is qualified in/trained to deliver to be a lonely and isolating process and was seeking to avoid or remedy that. Here, Henry was moved to a private space, downstairs, but as the only practitioner currently working on that level this left him feeling disconnected from the rest of the practice. I felt the same way during my time here, as 'my room' on site (both for writing 
and conducting interviews) was in one of the 'new' unused therapy rooms, downstairs at the clinic. Also, once back in the UK, I was also the only person working 'on my level' whether in the University or in my home office, and therefore felt similarly disconnected from the rest of my practice. Again, this improved once I re-connected to myself, something that Henry had already achieved long before I met him.

In terms of working within this current space, Henry still absorbs the setting he is in but finds that in this one he feels settled and relaxed, which manifests in his approach. He says he has noticed that patients are also more settled and relaxed now, which helps to make their Osteopathic adjustments easier. This observation may be one that practitioners in UK (particularly Simon) may want to learn from as this seems to support the changes that he, and others there, would like to make. This also prompts me to ask whether feeling relaxed and settled should be included as an indicator of CAM outcomes or noted within descriptions of methods or applications (in practice or research). Also, if this effect is noted beyond the practitioner and patient (i.e. the researcher), 'what does this mean for future CAM research?'

\subsection{Act 3, Scene 4: The miracle of everything}

The final story I want to share here is Victoria's. Victoria (not real name) is quite typical of the patients I met here in that she sought and arrived at this clinic as a 'last resort', having first tried everything that conventional medicine had to offer, without success. Such patients, as Victoria, are common across all CAM therapies meaning they pose additional challenges not faced in primary healthcare, both in term of the progression of their 'dis-ease' and their low expectations/loss of hope. This is often overlooked in mainstream critiques of CAM research and when used in defence of studies (to support findings) may be derided or dismissed as 'excuses' for failure.

Yet Victoria, and others with either chronic illness or zero-prognosis cancers, who may be expected to experience limited outcomes find that here, they can make complete/or near complete recovery. The differences in this presentation and performance must be closely examined, therefore, to learn any lessons within that Victoria and others have to teach about the potential to improve both study, and patient outcomes.

Victoria's narrative:

My mother saw the doctor on TV, talking about Crohn's disease. I suffer with this, so she insisted I come and see him. I have been to a lot of hospitals and seen a lot of Doctors, I even went to a specialist in Germany, they said there was no cause, and no 
cure, but all their advice and care just made me feel 'rubbish'. I couldn't accept that. I believed for a long time that CAM therapies couldn't help me, because that is what the regular Doctors said. I have been coming here for 3 years now. He began with the IDEL protocol and then I have had a number of different therapies, including Bi-Com, infrared sauna, osteopathy, detox, diet protocols, supplements and remedies. He seeks the cause and explains everything to me. He works with me, not for me. It's been amazing, like a miracle. The staff are all great too, always smiling, making you 'feel' like they care about you. They truly want you to improve yourself, and we have great rapport. The first time I came here, I felt like I was born again. I don't see my regular GP anymore and have recommended all my friends to come here too. Regular medicine just hurts you.

I think it's important for natural therapies to be in a natural place. A good environment is very important for the patient and this is a lovely rural area, a bit of an 'oasis in the desert'. In hospital settings you feel bad when you arrive and worse when you leave. I like everything about this Centre, and even the journey here is pleasant. When the setting is part of nature it makes you feel 'you are home'. Good energies are a product of this whole setting and composition, I feel secure here. And there are so many interesting items in all the rooms, there is one painting I particularly love. It gives a nice feeling to have nice and interesting things to look at, that also have nice qualities, while you're having therapy. I know that some reflect the Doctor's hobbies, and others are intended to impact patient therapy. This place and this practitioner are definitely part of the therapy. They are integral to the whole experience. A different setting would give a different experience. I would like to have a retreat here. They should have residential rooms, so you don't have to leave!

Victoria came here with low expectations of therapy and care, based on her medical history and previous experiences. This was transformed the first time she came here, feeling "born again" prior to even beginning formal treatment (she had the IDEL diagnostic protocol first). Something had impacted her that supported her engagement, compliance and outcomes which made her feel good. In terms of the whole performance of therapy here, the place and the people are the first factors that patients and visitors encounter. I had already seen that, in other centres, first impressions can influence expectations of therapy, but this was the first time I had seen first impressions create such direct impact. 
Of course, this clinic tries to be active in its dynamic effects. The awareness and engagement with Ley lines and geopathic influences, peace and tranquillity of nature, a Tachyon crystal temple and Tibetan singing bowl, even icons and artwork were evident from the moment I arrived at the centre in the intangible yet real way my senses immediately came alive with interest and hope. It took a while for me to uncover the myriad of reasons why this was so, as I studied both the people and this place in depth. Like Victoria, I did not know about either the presence or potential of these things but surrounded and supported by those who do, I learned that the only thing that limits these is our ability to engage with them.
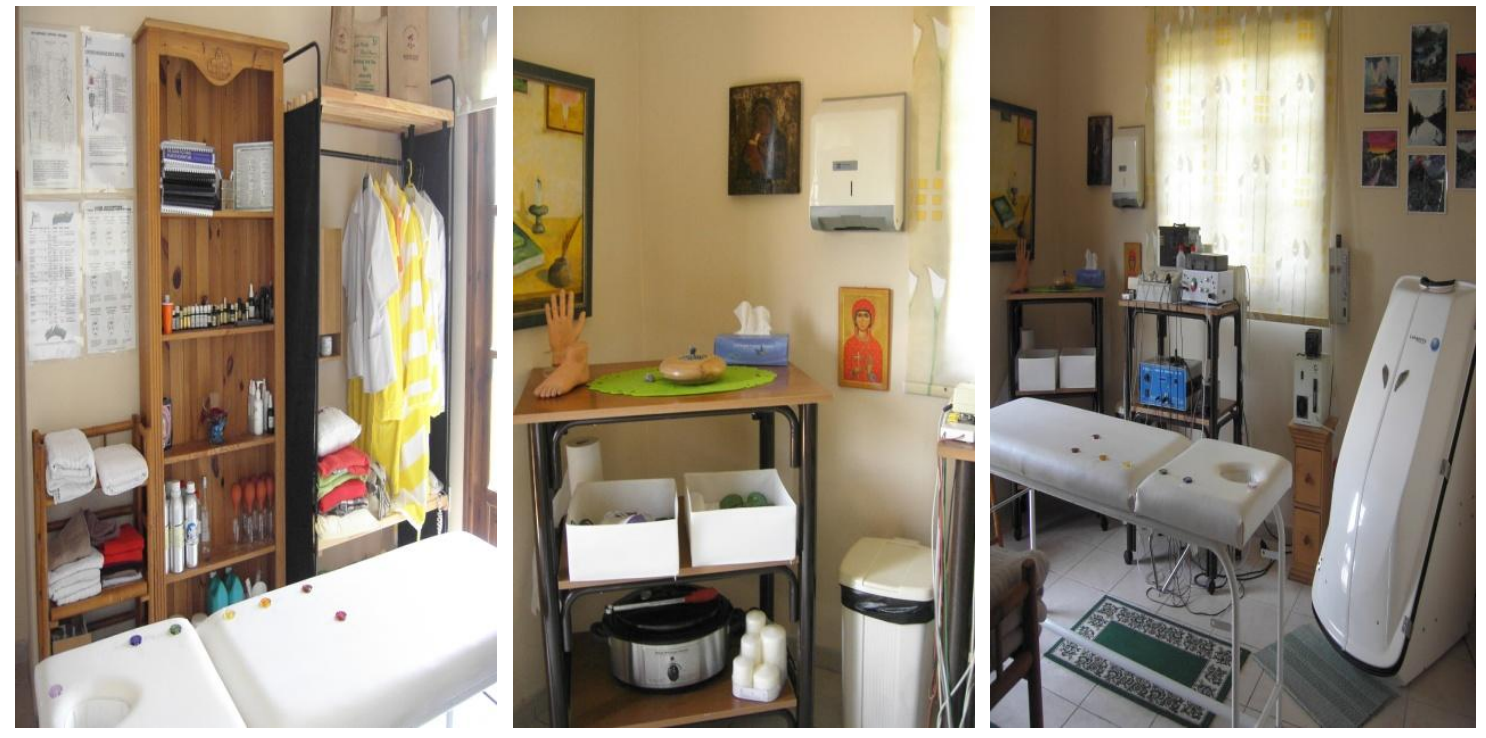

Triptych 8.9: Therapy room 1

As Victoria became more used to the clinic and the people here, she became proof that medical understanding and philosophies do not easily understand either her [illness] or CAM therapies. She had spent many years believing CAM's couldn't help her because regular medicine told her so when, in the end, they were the only thing that did. She became well as a result of the eclectic nature of her care here, which is why Victoria regards these people and this place as integral to her therapy.

Despite this 'miracle', the absence of solid research means it is hard to argue that anything offered within this clinic had a curative effect on Victoria. In fact, to even suggest this would be illegal (CAMs cannot 'cure'). However something (maybe everything) here did give Victoria a sense of relief and recovery from her symptoms. What that was or how it worked may not matter to Victoria, but recognising that whatever happens here is the result of the potential these people and this place creates, does matter. For me, this demonstrates a real problem with CAM therapies and CAM research. 
As highlighted in the literature (review in Chapter 2), CAMs are often presented in studies, or studied in sanitised, bland and generalised forms of therapy. Moreover their effects are classified as either relating to specific interventions or non-specific (placebo or contextual) effects. I have seen nothing at this site that could lend itself to study this way without seriously invalidating the processes, the outcomes or the research itself. Nothing I encountered here could ever be described as sanitised, bland or generalised (nor should it).

Unfortunately, until more is known about individual practices or the potential of these to inform either clinical guidance, or quality research, I do not see the situation changing. Coming here as I did, open to any possibility, I have found the potential of therapies exceeding my current comprehension and tried to create the 'space' within the study and myself to capture this. As an approach, this has been illuminating but also very challenging. I saw a lot, with my initial 'holistic lens' but I saw even more once I began to interconnect my 'holistic senses' and critique these acts as a participant within rather than foreign afar (a more common research a pproach). Like 'suspending disbelief' in the theatre, I began to see more of the 'reality' here once I stopped refusing to be mesmerised by the whole staging and performance.
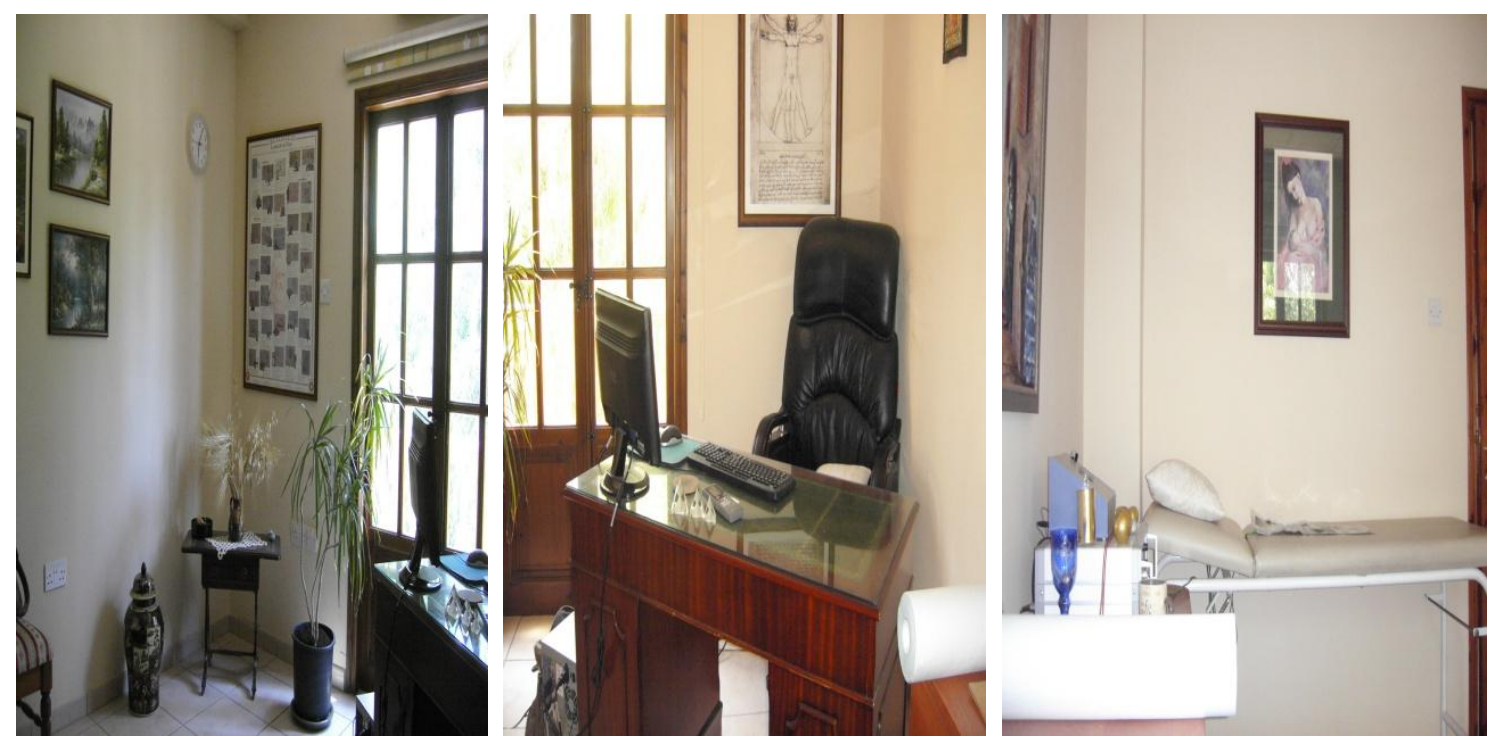

Triptych 8.10: Therapy room 6 (Bi-Com)

Similarly engaged, Victoria expressed many profound observations and emotions, often shared by others here particularly regarding the value of this unique location and setting ("an oasis in the desert") and her relationships with these characters and different modalities. Set against previous experiences of healthcare (Doctors made her "feel rubbish") characterised more by limited understanding and limited solutions, I see a real case for exploring 'colourful' 
approaches such as these more earnestly, with more understanding and more vigour. External audiences (like initial internal ones) may just be surprised.

\subsection{Act 3, Scene 5: Critiquing the whole (holistic) therapy performance}

From the opening act of arriving at the clinic (even the prelude of viewing the Centre's website) it has been clear that this performance is led by a strong character and that everything that happens here happens because of this one man (Holistic Practitioner/Guru). His vision has created this 'oasis in the desert', where patients and practitioners have the 'space' to truly experience wellness, in a haven of peace and tranquillity.

Another busy clinic, here the model is unashamedly alternative (almost the opposite of the UK site) and so embracing of holistic philosophy it could appear to some like a cult environment. A very different place, I found the potential for healing and relationships was also very different (something I almost felt before I even saw it) with a subtext of refuting the 'realities' of biomedical discourse by supporting patients with zero-prognoses, challenging the Medical Association and their methods and offering hope of another way to live through illness and become well/balanced.

At times an 'uneasy blend' of cultures, ideologies, philosophies and expectations (the practitioners all wear 'clinical' white coats, the walls are adorned with religious icons and humorous, natural and esoteric art and Tibetan singing bowls chime as Tachyon energised crystals maintain a 'healthy' force-field) this composition and staging suggests that there is space for all things and that all things are possible when we create space for them. Here, the 'space' is a direct representation of how the Holistic Practitioner blends his different influences (modalities, life journey, current context) into a coherent clinical model, with a protocol for practically every situation. Born from an embodied human experience (trial and error), rather than any specific philoso phy this practice has all the characteristics of a new kind of medicine.

Once inside, I found the power of this cult enveloping me as surely as those who come here seeking clinical care; first in the warm and open arms of the receptionist (like all staff here she was recruited for her sensitivity, caring nature and 'open heart') and then in the ways that every item, every person and every action felt like it was connecting me to another part of myself making me question my thoughts, responses and feelings. Sometimes this felt familiar territory like the emphasis on natural materials or chiming the Tibetan singing bowl, other times it felt 'bizarre' when participating in the daily 'smudging' ritual or observing therapy using 'historical' electrotherapy equipment (akin to images from old horror movies). These 
responses were typically felt rather than thought, as my mind and senses were stimulated together. I have to admit, I never quite came to align my thoughts and feelings with the thoughts, responses and feelings of the Holistic practitioner (cult leader) as those who commit to his care must, but I did see where my thoughts and feelings aligned with his (and where they differed), which was a powerful moment of connection.

Removed from the hustle and bustle of everyday life (and scrutiny), this overall scene was a great location to work. Also positively impacting the practitioners here, this place serves to support their own health and wellbeing while they also seek to create conditions where patients can heal. This is a similar observation to the Holland sites and distinct from the UK site, where (in the UK) the position and location of the clinic was primarily practical. In some respect, entering here felt like stepping through the looking glass into another world; a world where gardens are lush (despite the summer drought), bowls (Tibetan) 'sing', the Virgin Mary 'inspires' and against many odds, miracles can and do happen. It is as if the laws of society and culture carry no influence here, and the only factor limiting potential is the willingness to engage it. This sounds like a cult, and in some respects it is a cult (just as hospital is a cult, schools and Universities are cults etc) because participants here engage in a distinct culture with codes and rules that all who are initiated follow.

Engaging here as patients here do, I found remarkable people performing remarkable acts, in a place of beauty, peace, space and light. As an active backdrop, the whole production and performance, and the context and staging that facilitates this, is so unique and magical it has to be seen as a whole to be believed. Seeing and feeling this first hand, I revealed a compelling dimension to the stories of therapy here beyond the simple testimony of practitioners and patients. By 'suspending disbelief' I captured so much of value to this whole performance. Without this, my notes, recordings and observations of the variables of setting, personnel, experiences and outcomes would be at best incomplete. In particular, there is an unshakable spirit in the character of this whole production that becomes stronger each time it is tested (either therapeutically or legally). Patients come here and get the help they actually need, rather than that which the state or their health insurance will provide, and while this may bear a heavy financial cost, it is not so great that they would forfeit their health or their lives to it.

\subsection{Act 3, Scene 6: Reflecting on my research performance}

Here, just like the previous Centres, I found myself being caught up 'in the moments' I observed which provide the basis for this fresh perspective on CAMs and their practice. Almost from the outset, I found myself drawn into a culture so different yet reminiscent of 
others I have encountered on this journey, from the 'clinical white coats' to the presence of 'saints and icons' that it was easy to feel comfortable among the unfamiliar.

When trying to be critical and objective about these performances, I found making constant comparisons (things other Centres did better or perhaps worse, than here) was, in reality, of limited benefit and that this whole production needed to be understood as a totality to reveal its true/fullest value. This was challenging because, contrary to the UK site where I got 'bogged down' in the data, and to Holland where my spirit simply 'soared', here I became so 'dazzled' by the radicalism and results I witnessed that my data and appraisals bordered on fanaticism which made it difficult to be critical of the acts, and those who commit them.

This, in itself reveals lessons for CAM research as the effects of this staging and symbolism played as active role in generating my impressions, as they do for patients here, meaning I more often felt my responses before I thought them. Indeed, it was only upon realising this that I began to think at all (so blinded was I by 'the cult'). This effect was just as powerful each time I encountered the scene (both live and when working with my data). Of course, had I not been engaged at the participant level 'in the moment' by suspending my disbelief, I would have felt this less vividly and more abstract, with a critical distance. But then I feel (see, I'm doing it again) that sometimes engaging a critical distance can be the very thing that prevents researchers from getting close to what really matters. This is why I have also sought to maintain my own clinical (CAM) practice alongside my research, as much as has been practically possible.

I regard this rather like the difference between being empathetic and sympathetic, as a therapist. Being in 'that moment' I can appreciate how therapy feels, not just how therapy looks, which for a performance as complex and multi-layered as this has proved an integral strategy in creating a holistic picture of the event, for consideration and a nalysis. If I also think about it, I can see what I did and the lessons that I and others may learn from this. This example of 'space' creating the potential for relationships (Read, 2005) is as much about the space I observed in the clinic, the space created by my metho dology and the space I have been growing within myself.

Coming to this awareness has also brought me fresh conflicts. One of the most difficult of these was with my primary supervisor. While I have endeavoured, on the whole, to take on board his comments and advice regarding how my research may 'read', I have found his 'space' had sometimes led to him interpreting things I have written in a distorted fashion. For 
a long time I took sole responsibility for this (and this was likely justified) finding that I had either not been clear or made too many assumptions of the reader. However, towards my final submission, when I felt to have a clear handle on what I had done and how I felt about this, he was still misinterpreting me, changing his position regarding the stories I told. Most notably, he began to see Theo as a destructive influence, having previously been keen to meet this 'inspiring character'. This made me question the power of my writing in communicating the nature and events of therapy at this field setting, and the role of his 'contextual space', influencing his engagement with my data. This demonstrates the importance of 'frames of reference', both for those conducting research and those reading research. He had read a literature on 'cults' for example, that I had not and while his drawing the parallels with facets of my data was a likely enhancement, it also had the potential to skew the data into something 'dirty', that it was not. Was he concerned that as I grew, I was growing away from needing him or was he being protective and trying to keep me 'balanced' by putting ideas and observation into my head that were never there 'in the moments' on site, so I could challenge them and refute this view with confidence?

A risky strategy, particularly in the later stages of my research writing, this had the potential to disentangle my thinking and demolish my enthusiasm completely (as it had most definitely done in the earlier stages of my research writing, such that I ended up unable to write at all). In fact, I'm not entirely sure what saved me from this fate, except perhaps going back to the original data, putting myself back in the room (through re-engaging with the photographic images I collected) and really asking myself - what had I learned here?

Critical analysis, at this level, may mean really drilling down into the data to look for the different levels of meaning within it, but I don't believe that includes distorting the data from the intentions of those who created and performed it. I was not here to fulfil some twisted ideal and 'prove at point'. Indeed, I had no preconceptions of what I would find prior to coming on site here - how could I, I had never experienced anything like this place on earth before! The issue then, is not why or what mechanisms of control may have caused me to be 'blinded' in my engagement, but to recognise this was the 'reality' of therapy here and that this may be a factor for future trial designs and evaluations to consider. 


\section{Chapter 9 "A Tale of Two Clinics"}

The final layer of my fieldwork was conducted in Denmark, Northern Europe. A city of arts and culture, history and entertainment, here street artists and prestigious collections of art and sculpture sit side by side amid the waterways and bridges that provide both the means and the method for residents to perform this lived production. Presenting a different pace of life to the sites in Holland and Cyprus, I found the nature of many people's acts here to be more practical and 'rushed' (more like UK), in both the ways their rode their bicycles and their approach to engaging therapy.

Effectively 'a tale of two clinics', here I worked with two, small independent therapy clinics, each located in different districts of a large city with their own character, architecture and populations. Delivered by a solo therapist with no assistants (a do minant clinical model here), each offers a range of therapies direct to the public, for either general wellness or health specific issues. At Clinic 1, the primary modalities include zoneterapi (reflexology), organic massage, Bowtech and Hopi ear candling, while at Clinic 2, the main offerings are zoneterapi (reflexology), cranio-sacral therapy and Reiki.

To aid a sense of the physical space, set plans are as follows:

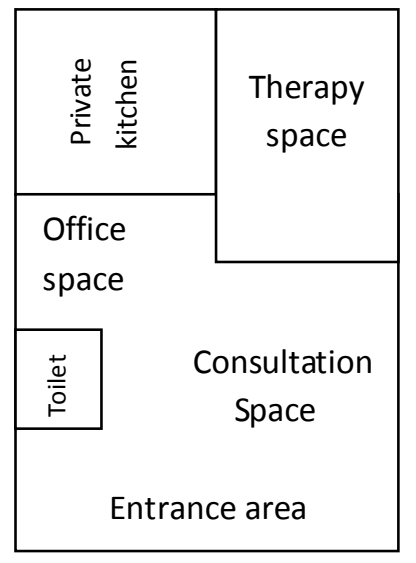

Set plan 9.1: Clinic 1

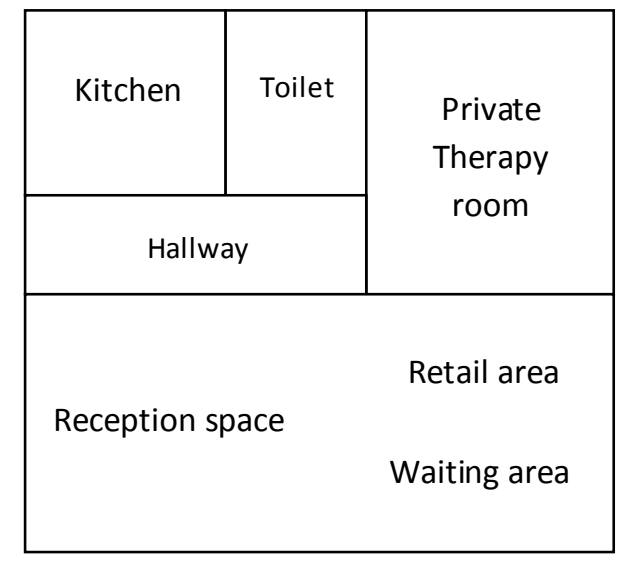

Set plan 9.2: Clinic 2

Across three and a half weeks duration, I split my time equally between these clinics and observed several treatment sessions, completed two practitioner interviews and also five patient interviews. As solo clinics (rather than a group practice), I was concerned my time in this location would generate sufficient data and wanted to guard against the possibility that illness, for example by a practitioner, could reduce my sample to nil. However, this did not 
occur, and between these two I generated a data yield of 7 pages and over 3000 words of scripted dialogue, and a supplementary field notebook. The following scenes represent the most pertinent stories and themes of patients and practitioners from both sites, which illustrate the nature of care and relationships I found here, in therapy and research.

\subsection{Act 4, Scene 1: A critical touch}

Clinic 1 is situated within a trendy business district most popular with young professionals. A relaxed and bohemian area, with artisan bakeries, bespoke jewellers and a small park nearby, this feels a very supportive place for a therapy practice to be, and is why the therapist who works here chose this place, after rejecting many. Not the only person to work here, she sublets the clinic from another practitioner and has exclusive use of it for two days per week; her clients typically attending for help with musculo-skeletal issues, soft tissues injuries, general aches and pains and stress-related complaints.

Isa (not real name) is a client (they are clients here, not patients) whom I met at the outset of my fieldwork here when she brought her mother into Clinic 1 for therapy. A week later, she returned for her own therapy, and participated in both a treatment observation and interview. On the day I observed her, Isa was receiving a Bowtech treatment.

I was already inside the clinic when Isa entered from the street. She knocked on the door (this was locked in a new security measure having been recently robbed) and Josefine (therapist, not real name) went to let her in. Isa and Josefine chatted freely as Isa walked past the two wicker chairs and the large window to behind the heavy cream curtains (that screen the interior from the street). Josefine followed her, once she had locked the door again, into the open consultation space behind the curtains where they sat together warmed by the candles that Josefine had now lit, and discussed how Isa had been since her last visit. The space felt intimate and warm as these 'equals' connected, with the world locked out! Then, in synchronicity, they moved into the treatment area (this felt like a 'cosy wine cellar') where Isa took off her shoes and climbed to lie face down on the bed. Isa remained clothed during the treatment, in a lightweight summer dress, covered with a couple of thick, warm blankets.

Over the next 45 minutes, Josefine performed a series of moves which involved uncovering an area and applying deep pressure (using thumbs) to trigger points that 'send' messages to the brain to relax and rebalance, before re-covering and moving on. Between each move, Josefine stepped away from the therapy area (and sometimes beyond the curtains), returning a few minutes later to check for responses. Her technique was hypnotic to watch, methodical and 
totally focussed. Dressed in loose sports-type clothing, she looked like she was performing some kind of martial art as her choreography blended with the soft background music. In this 'space', everything and everyone else ceased to exist.

Joesfine had just completed a move to Isa's right leg when Isa suddenly took a long deep breath, and her toes started to twitch, uncontrollably. Josefine stepped back and observed as the twitch developed into a wriggle, finally settling into another deep breath. A few moves later, to Isa's upper back, and the same thing happened again, more twitching and wriggling in a visible, subtle relaxation of her body. Josefine continued to move around the therapy couch (and Isa's body) applying pressures then stepping back/withdrawing to wait and watch for Isa to respond, which she always did with deep breaths, twitching and yawns. Isa's whole body seemed unable to resist the stimulus of Josefine's technique, in the end resulting in such a total state of physical and mental relaxation that when Isa completed her final stretch (before getting up) she was 'like a lion who had just awoken' from a very deep sleep. While Isa was 'coming round', Josefine again withdrew (this time to write her notes) and waited for Isa to sit up slowly, and get off the couch.

I was transfixed. I had never witnessed such a powerful treatment. In this soft lighting and simple space Josefine and her therapy were like one medium, sea mless in their application and potent in their results. After Josefine and Isa had completed their accounts, fixed appointments etc, Josefine left the clinic to get some coffee, giving Isa and myself some privacy for our interview.

Isa's narrative:

I have been a client with her [therapist] for around four and a half years. I saw her first at home, and then followed her when she moved here. When I first came, I had zoneterapi. I knew of this from having treatment from a Dutch therapist in Egypt, and when I looked in my locality here, she [therapist] was the only one who could see me straight away. I suffer with back pain, and have lost faith in the doctors to help me. I got zoneterapi, twice a week for a long time, then one day she [therapist] suggested I try massage, and I immediately got better responses. Now she [therapist] uses a combination of zoneterapi, massage and Bowtech, and it really works. She [therapist] is only a little person, but she has strong hands. I often get an emotional release too, we laugh a lot and I cry. I have a good relationship with her [therapist], and I trust her. I think that's really important. 
I don't feel this particular setting has any direct effect or influence on my physical outcomes. Perhaps this matters more as a first impression, and I think a good setting can influence your decision whether to return for more treatment. The location is convenient, and the transport here is good. I always come by bus. But for me, it doesn't really matter where therapy happens as long as it is this therapy with this therapist. She [therapist] helps me when the doctors couldn't. I depend on her for my survival. Sure, everyone wants more luxury, but the space is not my focus. Really, this is just a room, in a convenient location. I do like the music and candles, they make the space 'warmer' and less 'clinical'. I used to see a chiropractor in a more hospitalised setting. That was cold and sterile, and I didn't really like that. The chiropractor was actually a bit of a hunk, which meant I often felt self-conscious in front of him in my underwear. This made it hard to relax, and probably had a negative impact on my responses to his [chiropractic] treatment. With her [therapist], it's different. I can be myself and not worry about such things. This is much better for me.

I found a number of things striking about Isa's narrative. Attending mostly for physical outcomes, Isa credits the unique qualities of this therapist (her training, her skills and persona) as responsible for these. This is a sentiment expressed in previous sites and something I get feedback about within my own clinical practice. Finding a good match for her needs, Isa associates her positive outcomes of therapy with this character more than any other factor. However, Isa also reveals that together, they laugh and cry, suggesting the power of this therapist's 'touch' may by deeply physical but not only physical.

The depth of work applied to Isa, and others, says a lot about how much this therapist genuinely 'cares' about her clients and puts all her energies into their treatments. I saw this clearly in the focus and hypnotic method of her application and the way she locked or 'zoned out' any possible distractions to their union, in therapy, and even experienced this when she delivered me a 'taster' session of the same. Using this 'insider experience', I found I also focussed on the therapist and her skills, as together she caused me to surrender to the act and permit my response. She is, as Isa noted "only a little person, but she has strong hands" and this critical fact meant I barely noticed the place or the music she played, as what she 'did' mattered more. Reflecting on this, I am unclear whether her 'zoning-out' the space was so infectious to me that I did this too, and if this was an active part in our union. Alternately, was her technique/therapy such that it was hopeless to remain in a single level of awareness while receiving it? For the third time within this study (the first two being in Holland) I had found my 
initial observations being deepened by active participation, neatly dramatising the difference between 'critique from within' rather than 'foreign afar', which challenges the traditional rejection of subjective research as being limited in value.

As for the qualities of the 'space' itself, I found this setting both objectively and subjectively supported and communicated this therapist's aims, creating more 'warmth' than a 'clinical' setting and a place where each could relax and be themselves. Within this, the therapist felt able to guide treatments as she saw fit (for example, moving Isa from zoneterapi/reflexology alone to a blend of zoneterapi/reflexology, massage and Bowtech) and forge her identity as an autonomous, caring and committed thera pist.

Seen as independent of the place she practises, Isa is clear that she would not gain the same approach or response from any other therapist, regardless of the nature or qualities of the setting. Therefore she will always prioritise seeing this therapist over the place where she works and so considers issues such as ease of transport and luxury to be 'enhancements' to the event rather than integral to it.
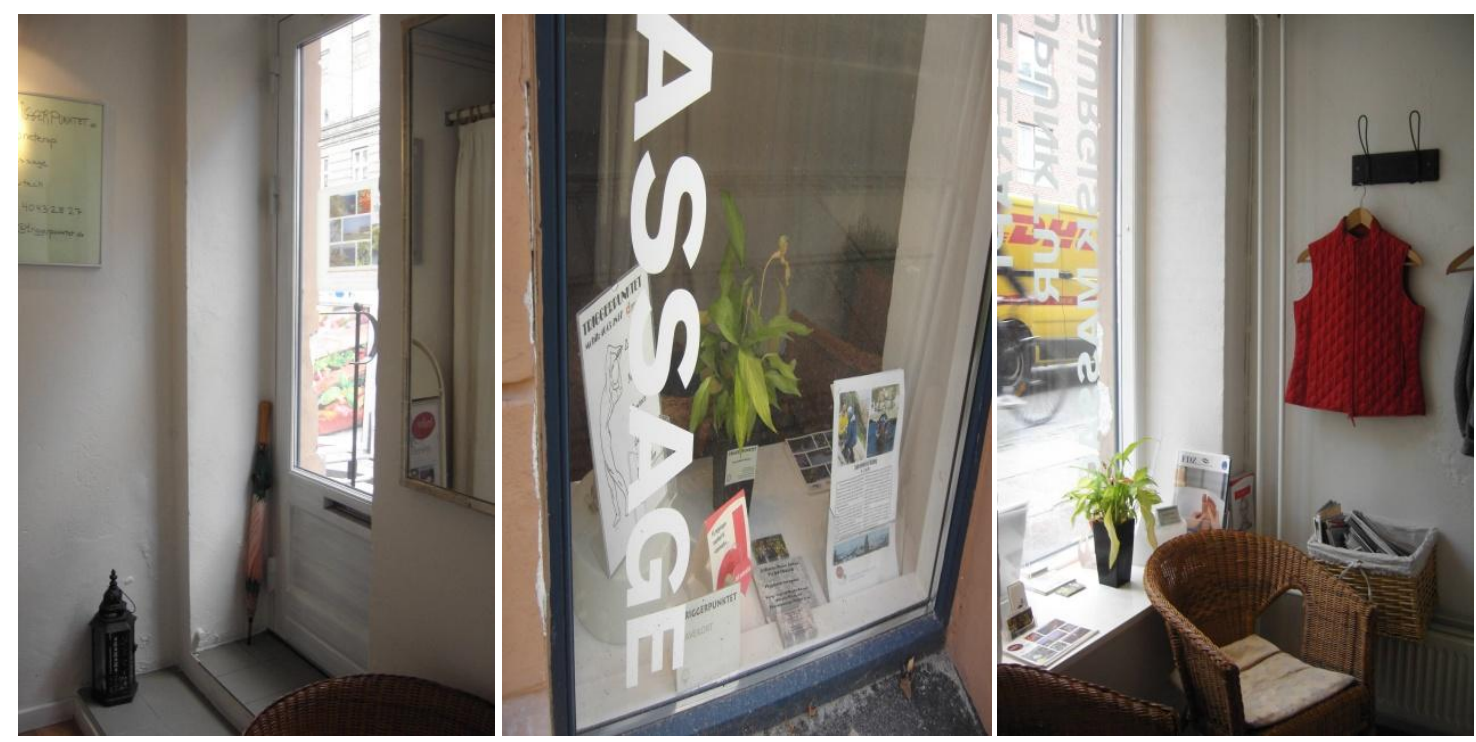

Triptych 9.1: The entrance and waiting spaces at Clinic 1

Transcending the space they share today, I found every move, every pause this therapist makes to be careful and considered. By this point in my study I had seen many therapists at work; some who seemingly 'went through the motions', others who sought to connect with their wider surroundings and engage these as active parts of the whole event. Here the approach was more to focus inwards on creating a 'space' for the therapist to feel the patients/clients needs and to meet these (sensitively and intuitively). Guided by a basic prescription or routine, the intensity and flow with which I saw her deliver it always went 
beyond this. I believe this is what Isa was talking about when she said she 'depends' on this character and 'trusts' her.

Isa is an established client here, so her tolerance, boundaries and expectations are already known by this therapist and this lends some of the confidence in 'depth' I observed. A less established or open relationship may (as with my own practice) result in a different approach, however during my time here, I only observed acts with pre-existing clients which will have pre-disposed me to witness similar performances and therefore created potential for me to make such observations about 'confidence' and 'depth'. Within the nature of their relationship, the duration and confidence of each in the other became the focal point with other aspects only framing this. This is further highlighted in Isa's final observation about therapy with the 'hunk', adversely affecting her confidence in their relationship and imposing limits to the potential of treatments/outcomes.
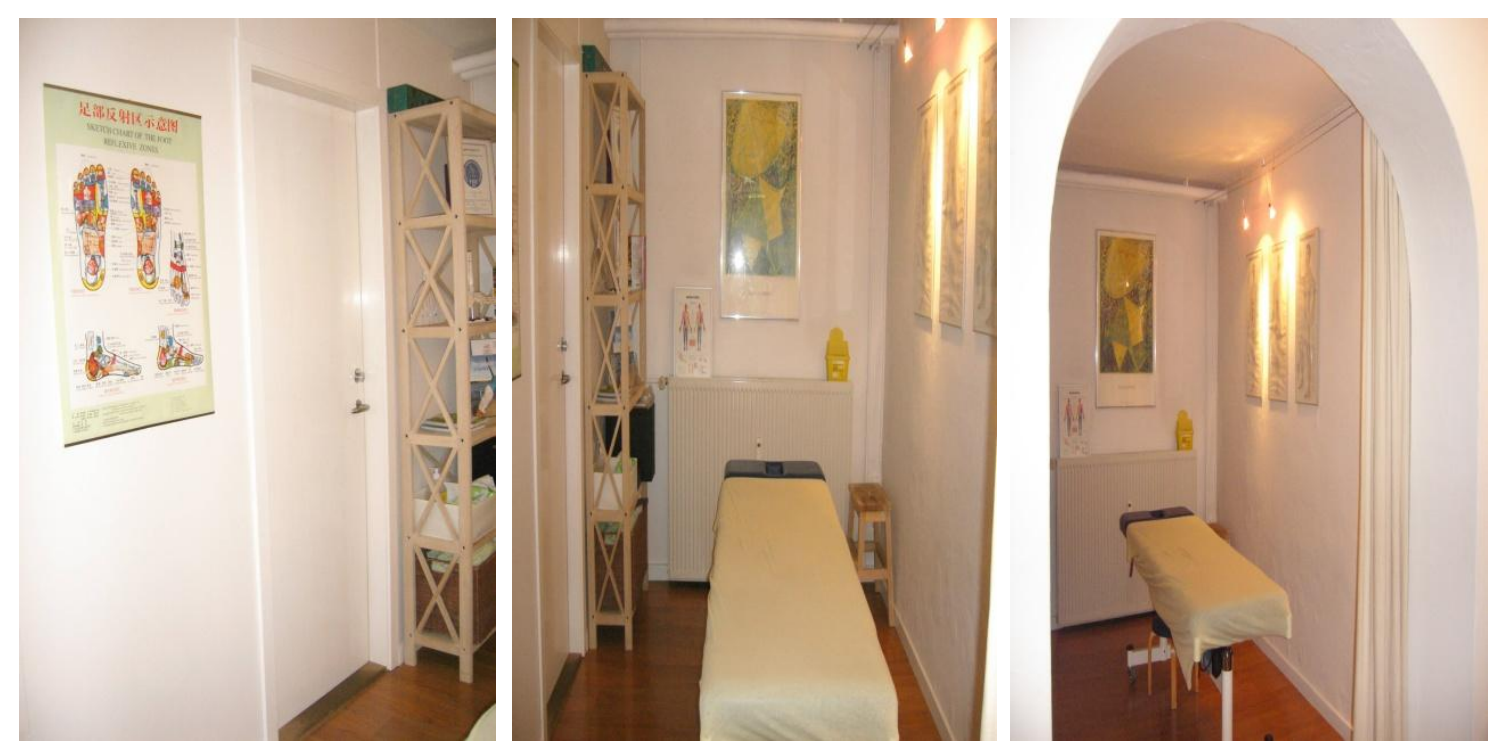

Triptych 9.2: Therapy space at Clinic 1

This was something I had never considered before. Indeed previously, patie nts I interviewed have said how it is important to like their therapist, to be able to relax and connect with them. The idea that 'liking' your therapist 'too much' can create inhibitions and prevent good responses is one that may warrant further investigation as, according to Isa, the potential for impact is significant and trials should at least be aware of this potential. The ability to mediate this may also be a matter for the individual interpersonal skills of the therapist, with some CAM therapies more at risk than others (i.e. those where undressing and intimate contact may be required). This is not, however, something I was directly aware of within this particular study. 


\subsection{Act 4, Scene 2: The lone(ly) therapist}

While therapist and clients build strong personal relationships, in support of therapy, here at Clinic 1 I observed the relationship between practitioner and place to be rather less successful. Josefine (therapist at Clinic 1, not real name) began this therapy clinic working from home and mobile in office buildings. She moved here later, in a bid to develop her business in a new direction after the global financial crisis threatened her office contracts. Alongside attracting new clients, this location provides the opportunity also for her 'office clients' to visit her privately and so continue their existing therapy programmes.

Over our time together we shared a number of informal conversations which I reminded her of as we completed her interview, so the narrative I present here could include aspects of these too.

Josefine's narrative:

I always wanted to train in zoneterapi, ever since I had a boyfriend who did this when I was younger. At the time I couldn't afford to take the course. Seventeen years later, I had the money and did the course. I really enjoy doing this work, and now have extra training in Organic Massage and Bowtech too, which I hope will make me more profitable. I would describe myself as an alternative therapist. I'm not interested in wellness. Wellness, here, means just for luxury, and what I do is not a luxury treatment. It is a healthcare intervention. I also do gua-sha, which is not really popular, and the hopi ear candles. Of course, when the candles work, people don't need to come back! I think the most popular is the reflexology, because it is subsidised. The other therapies may suffer because they are not, even when they can be better for the client's problems.

I do some of my work here, and some in businesses, giving treatments to their staff. When I work there [businesses] I always try to make the space better, if I can. I take flowers and candles and my music. I think I could do this work anywhere. It was part of my training, how to 'zone out' distractions and focus on the client. I take this deep breath, and put my own concerns aside. I think that having some control, and feeling good about space, is important to therapy. It lets the place become a large part of therapy, with the potential to impact the whole experience, from when they come in to when they leave. I get frustrated by the limitations I have with the companies. I would like to give longer treatments, and I ask them to pay for these, but they won't. So the 
workers end up getting either a superficial or focussed therapy. When I am here I can do what I like to do, and give longer, more holistic treatment. I think time is more important than the place. What I do here is better for the clients and more satisfactory for me. I am in the happy business. When I am happy, I make people happy!

I picked this location because I thought it would be good for my business. I felt very positive when I first came here. I thought this place had good potential. It has good transport links and is close to a number of office buildings. It was also available at a good price and was not one of those esoteric places. I don't know how to talk to those people and would not feel able to discuss cases with them. I actually rejected many places before finding this one. I think it feels like a wine cellar. It's cosy. People may say they like the picture, but I can't talk about it. It's not mine and it means nothing to me. There are many things here I don't like though, and can't change. The rug is ugly, I don't like the colours, the curtains or the dried flowers that she [the owner] puts everywhere. I feel like I am fighting this interior sometimes. I would prefer fresh flowers. I was attracted to this clinic, partly because I had a good feeling about the owner. She appeared supportive and gave me the impression we would be a team. I didn't want to feel alone here, but this is exactly what I am. We have no communication and no compromise over the layout or presentation. Now another therapist has joined the clinic, and there is a new dynamic. I am not sure this place works for me anymore. I would like a better sign. This is the 'secret' clinic. No one knows we are here until they come. Most people find me on my website.

I like to wear my own clothes for work, anything else would not be me. I wear mostly sports type clothing, and find I naturally attract more sportspeople. I would never dress like a nurse, because I'm not a nurse. I find uniforms 'sterile'. I find the business side hard, and don't like to push my treatments. I do have real financial concerns though. The global crisis has hit my business hard. The companies say they can't afford me now. I may have to close the clinic.

Josefine's story, much like my observations of her, reveal how passionate and committed she is to this work. Always acting with the best of intentions and to the best of her ability, she seeks to provide an effective solution for her loyal clients, for the problems that life serves them with. Describing herself as an 'alternative therapist', she presents a definition that I actually found to sit somewhere between alternative and regular medicine, being neither option as I understand them. More complementary than alternative, perhaps, Josefine 
chooses not adopt the clinical whites seen in UK and Cyprus and dresses instead a more relaxed way, like Holland, applying therapies that have both physical and dynamic effects. Like much of CAM, Josefine projects an unclear identity, knowing there is something more than conventional care but unsure how to locate this within the prevailing culture. Choosing 'healthcare' over 'wellness' is the best distinction she can make (wellness means luxury here, and she know she is not this!) as she struggles to identify with more 'esoteric' people and places, not knowing how to communicate with these.

There are many aspects of Josefine's story that resonate with my own; not so much as a CAM practitioner but certainly as a researcher of CAM. Her narrative reflects the optimism and despair she has found as the reality of this therapeutic dream, and its effect on the satisfaction and overall sustainability of her work, begins to slowly but surely unravel. A story of inner turmoil and mediation, her strength and fragility has been tested and eroded by her situation, despite hiding any outward signs from her clients. A victim of circumstance, perhaps, this has been compounded by Josefine's choice to expand her practice in a challenge to the Global Financial Crisis and people's a bility to engage in private therapy.

The parallels between this, and my own story of research, are compelling. I too have found my initial therapeutic dream to be harder to sustain than I first thought. Also subject to external influence, I have experienced both highs and lows on this journey yet tried to present a constant face, publicly. When this happened to me I found it difficult to see where (or how) to carry on, and naively tried to bury myself in my work, only to keep being pulled away from it by one family crisis after another. I have touched on this previously and so do not need to delve into 'chapter and verse' again, however, I recognise now that limiting the space within myself to admit and address my 'reality' may also have limited my potential to survive it. This is the situation I also found Josefine in, when I met her; earnest and desperate to serve a world that she feels needs her, hoping or waiting for things to improve naturally.

Both a potential catalyst and casualty of this, her relationship with this 'place and space' became the central issue, and one that she vowed to address, after I left. I do not know if she managed to resolve or improve her situation, but I do know that while I was with her Josefine had a need to feel supported in her work that was not met here. Moreover, her relationships with the others who share this place (on days she is not here) had proved a disappointment to her. There is something in this about both the ideas of expectation and satisfaction expressed by patients at previous sites and how a mismatch can lead to poorer reported outcomes (e.g. Pete on 'fancy clinics' in Chapter 6), and also the role of external support versus internal 
conviction, again seen in previous sites (Theo in Chapter 8 clearly had this, but Simon in Chapter 6 did not and the whole team in Chapter 7 held a delicate balance), on the practice of therapy. Here, Josefine presents a different dynamic in that she has conviction in her work but worries this will not withstand the external pressures around her, so seeks extra validation.

Like Josefine, I also hold inner conviction but worry this is not enough to sustain me in the face of external pressures and politics. This is perhaps why I feel the need to expose and explore 'space' as a central issue within my research, so that I and others who feel this too may learn how to understand and survive this situation, and just maybe find a solution or development. For Josefine, living 'between philosophies' means she presents yet another therapy dynamic, different to those seen previously within the study, and with potential for different therapy performances and/or outcomes than previously observed.
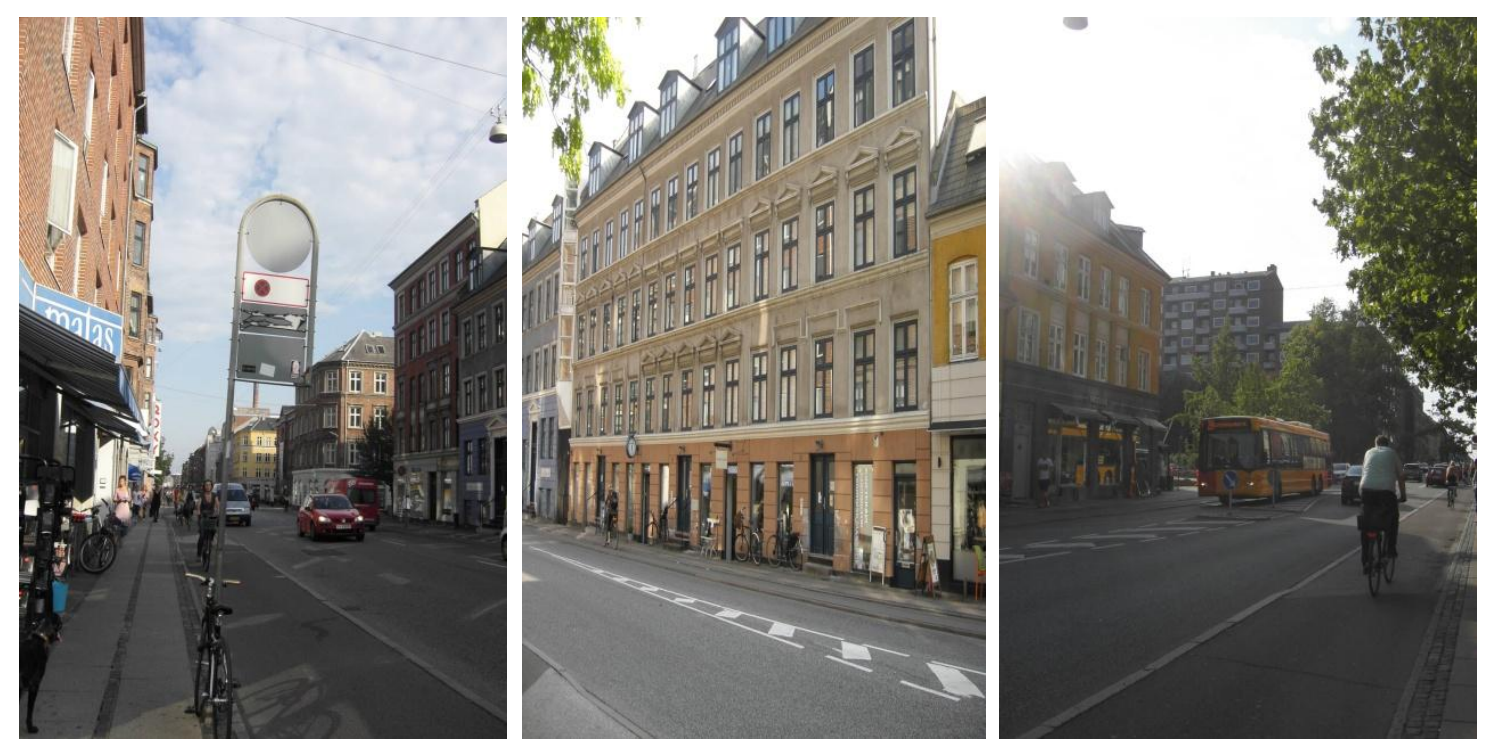

Triptych 9.3: The exterior at Clinic 1

Set among a very 'crowded market' (due to state recognition, there are small therapy centres like this all over the City) this may also be why she has struggled to develop her practice into the thriving business she wants/needs it to be. Certainly, joining forces with other, likeminded practitioners (shared sub-let) has not helped as each has failed to connect in any meaningful way, limiting the potential of the whole group. Indeed, at the time of Josefine's participation she was reducing her commitment to the verge of leaving completely. This was a shame, as I found her a very talented and highly motivated therapist (she gave me some amazing Bowtech!).

Within this 'shared' space, (other independent practitioners use it during other days of the week) I found limited personal investment from Josefine, which reflected both the lack of 
relationship Josefine has with this and her commitment to a future here. She did like to introduce some (soothing) music and candles to calm this whole setting, encourage silence in clients and allow each character to focus on their own role, but these were not permanent fixtures as she removed them prior to handing the space over to any other therapist. This approach reminded me of the 'tricks' the reflexologist in UK employed, to also humanise a space that was not hers so that both she and her clients had better potential for relationships.

I said at the time, this was how I would have to adapt the UK space if I were to work there although I meant to help me connect better to, and relax within the space. Indeed, when I used to sub-let space within a primary care (GP) practice two days a week, this was how I used to apply my modifications. A distraction rather than a connection, this was also at a time before I conceived and converted to holistic philosophy proper (and where I am inclined to situate Josefine and her practice). For Josefine, these additions do better root her in this space but only as a place from where to disconnect as she places herself somewhere only she and her clients exist. Described as 'zoning out', Josefine adopts this in her office treatments too, even though she claimed that developing a 'base' would provide more opportunity to deliver a more integrated care. An opportunity, perhaps, she has yet to fully act upon.
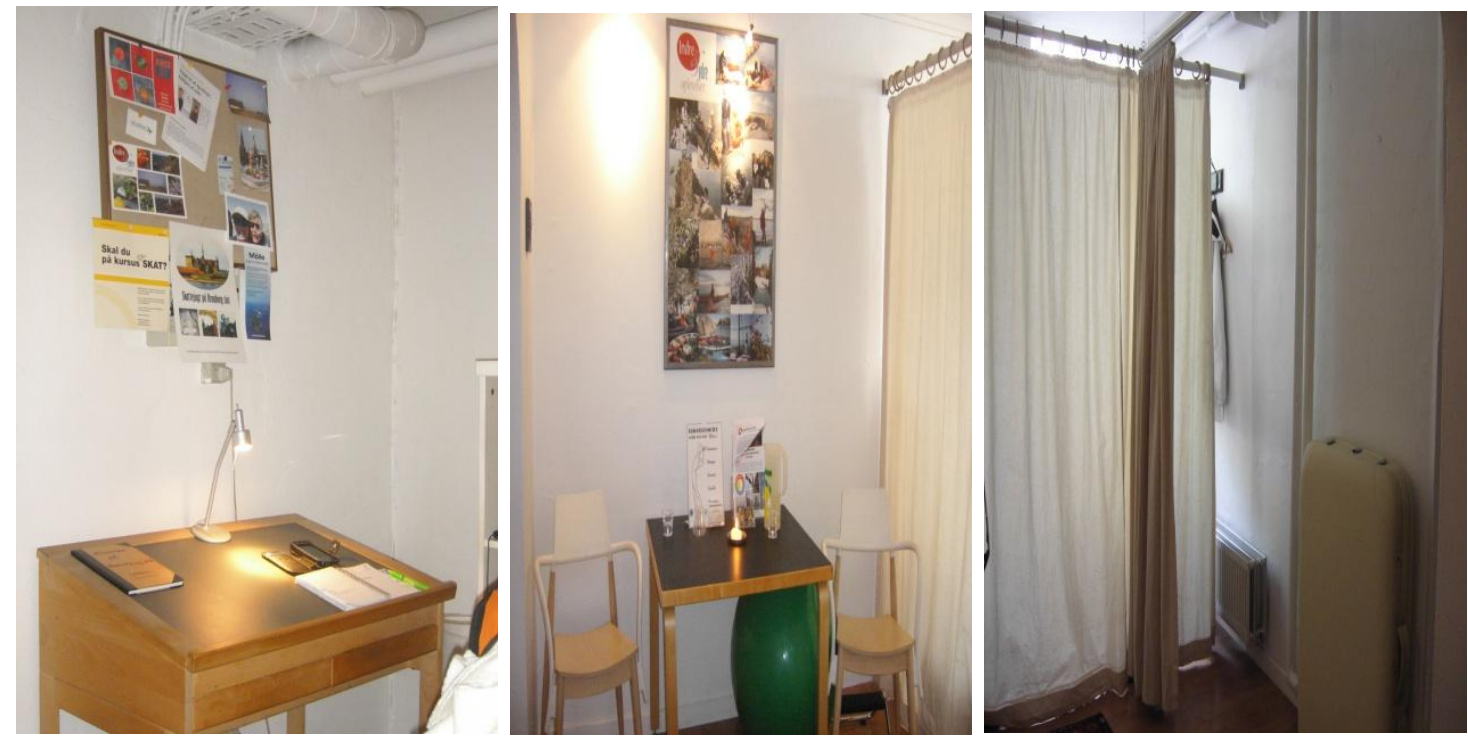

Triptych 9.4: The office and consulting areas at Clinic 1

This is an important distinction between 'spaces' that I had not realised until now. Space has, in every place I have visited so far this study, seemed to create the potential for relationships I found in them, encouraging different layers of engagement with people, place and plot. This could be by forcing the personal relationships to the fore, or the mechanics of therapy. Alternately, it was about awakening different dimensions of the self and 'moving the spirit'. 
However, engaging with Josefine's story and the experiences of clients I observed with her, I find that her space is used to disconnect characters with their everyday lives (the real world) so they can enter a place of therapy and response unimpeded. Josefine explained as much to me when I asked about her use of music, stating that she liked to discourage clients from talking/chatting during treatment, as this helps them to better focus inwardly, on themselves as participants and solutions to their lives/problems.

The qualities of this space may make this an easier task (not much here to hold or engage you) but this psychological tool, supporting an internal holism rather than a universal one, poses some contradictions to the other models so far appraised within this research. More important than this, perhaps, my time here achieved a very candid friendship with Josefine and I felt that participating in this study, and specifically completing this interview, created a 'space' where Josefine could really expose and explore the issues she faces within her practice and that she has long been aware of (and unhappy with) but felt powerless to challenge. Again this provides support for Read's (2005) assertion of space creating the potential for relationships. Here, given the space she needed, Josefine started to develop relationships within herself; exploring her authenticity, what she really wanted and exposing her potential pathways to achieve this.

I suppose it is hard to invest in a space that does not feel like 'home', or where there is such resistance or challenge, that it can feel hard to earn your place there. Again, this bears real resonance with my research journey that, like Josefine's conviction she had found her space, has become eroded over time. When I began my PhD, I was one of seven postgraduate students working on CAM related studies, my department had its own CAM professor and the Head of School was also keen to support a CAM theme within our research strands. Within a couple of years personnel changes saw our CAM professor leave altogether and our Head of School promoted out of the department, such that the PhD students were all that was left and as they, one by one, began completing their studies I began to sense that our time here, like other rare species, was heading for extinction. Moreover, I noticed how these CAM studies were becoming more mainstreamed, in both ambition and execution. The dynamic had changed and I wondered how long this place could continue to work for me.

As I said earlier, like Josefine I had felt external validation would be necessary to support my inner conviction, in the longer term, but as this was slowly withdrawn from within my University department so I began to also withdraw from it. Almost pre-empting the end of any relationship, I began attending (and therefore investing) less, rationalised that it was more 
'cost-effective' to work from home (a conclusion Josefine was also considering) and in doing so created a more 'isolated' space for myself than where I began from. Around the completion of my third year, I recall my supervisors asking how I felt about nearing submission and leaving the University. At the time I hadn't known how to respond, I didn't really want to think about it. I had really enjoyed participating in academic debate inside and outside the University and the prospect of being 'cut loose' and cast back 'into the wilderness' felt almost like bereavement.

Carrying this through my remaining time has been a heavy burden, impeding my ability to act effectively and create potential for success. For Josefine, the scenario is different but the outcome may be the same. She, like I, had tried (to find a home) and felt to have failed. For me this marked the onset of a period of depression (a rare outcome for one generally as upbeat as me). I only hope it did not have the same effect for Josefine.

A further interesting 'side-effect' to these performances, which I did not recognise for a long time, was that my writing of them also highlighted my growing disconnection from self (very different to earlier in the study) and I began compartmentalising rather than connecting the value in the data. Again I rationalised (using my thinking or objective rather than my feeling or subjective self) that maybe something about the values assigned to a space by those who inhabit it can 'infect' the levels of connection any onlooker may have. I saw elements of this too when I got 'bogged down' in the concrete detail in UK and my euphoric responses in Holland and Cyprus and wondered if this could perhaps be more apparent during formal observations, when I was describing what I saw, and that a lack of any obvious relationships with the space, meant I could not describe those. As I reflect now, I wonder if this was also what happened to my colleagues, as both their ambition and execution became more mainstreamed (or realistic?). Again, this dramatises additional value that can be gleaned from 'a participant within' perspective that a foreign afar would miss.

\subsection{Act 4, Scene 3: All by myself}

Presenting a contrast to Clinic 1 in some ways, yet similar in others, is Clinic 2. Located in a different part of the City and a more residential area, popular with families, Veronika (not real name) also works within a small, lock-up shop at the foot of an apartment building. She initially began her practice from home (like Josefine) offering zoneterapi (reflexology), craniosacral therapy and Reiki part-time, alongside her previous job in a bank. She moved into this Centre in order to make the leap into full time therapy as "having a shop in Denmark is seen as a real sign of success, clients think you must be good if you can afford a shop'. Taking this 
premises on was therefore a strategy to bring in more business (rather than replace lost business), and sustaining her practice for the longer term. Unlike Josefine, Veronika has found these selections and motivations to be a 'positive affirmation' of her personal convictions and her confidence has grown as a result.

However, she still suffers the same isolation and loneliness as Josefine reported. Greater, in fact, because she neither has the support of other therapists around her or her family (they think she's weird because she has different ideas/philosophy to them). Seeking wholeness in life and through therapy is a difficult task, made greater by the dominance of established social ideas and intolerance of challenge to these. Like me, Veronika sees engaging in this study as a start, bringing greater clarity and understanding to the future of CAM so we can all be better.

Veronika's narrative:

My life used to be very different. I worked for a long time in the bank, and I enjoyed that, but then mum died from breast cancer when I was twenty-nine, and one year later, my two aunts were also diagnosed. It made me look hard at my life, and I thought I have to make changes. I took a nutrition course first, and became very interested in alternative thinking, so I studied zoneterapi next. I never experienced this before, but it is the most popular therapy in Denmark. As I developed, it became very difficult to continue in the bank. My new thinking, and that world, just don't fit well together. I had to plan it though, and it took me five years to finally leave there. My last year, I was there only in body. It was unbearable. So I took some time off and walked the Camino, in Spain. I was so happy there. In the Camino, I got to connect with myself, with others and the environment. I was free. The bank was a prison. When I came back, I knew I had to quit my job.

I began using just reflexology, and then started my training in cranio-sacral therapy after a year. I had a lot of gaps. I think some courses can be a bit of a money-making machine. I wanted to do treatment on the whole body. I think these therapies complement each other well, and together make a 'whole person' approach. Reflexology is the most acceptable therapy in Denmark, and is viewed by many as 'not so alternative' anymore. This gets people into the shop. But a lot move onto craniosacral, if I think it's better for their problems. I use some reiki too, but not on its own. That wouldn't be acceptable here. I was trained to work mechanically, but I practise personally. My former work, in the bank, helps me identify with many of the people 
who come here, and I apply my spiritual learning in connections and interactions. If I can't make a 'connection' then I feel the clients are not involved, and I am only delivering a treatment. Those clients will not respond so well, and may not come back. The people who respond well to therapy are usually those people who respond well to me.

I set up this clinic 6 years ago. I just looked across the street from my flat, one day, and saw this shop was available to rent. I always thought that if I should work anywhere, it would be here. It was fate and I love it. I think having a shop is important for my image. It gives people faith in what I do, they think I must be good if I can afford a place! Everything inside here is mine, and it is here for a reason. I didn't really think about it too much. I just did it and it feels right. I actually think I work differently here. When I worked at home, I felt like I had to prove myself more. I value myself higher here. I think the clinic is staged well, but I do worry that clients will judge me, by 'reading' materials, artefacts and my tastes. I suppose that 'I am everywhere' here. People may think I'm crazy, but at least I am free to be myself, and I think that gives this place a good energy.

Placing herself in the centre of this narrative and the community she lives, I saw many connections in Veronika's story that play out in her work and the energy she gives to it. Hers' is a passion, just like Josefine's, but with the addition of both a traumatic life event (her mother and aunts) and an epiphany (the Camino) not unlike other practitioners who have an illness or pain and find solace through a CAM therapy, which they are so convinced by they go on and train to deliver so other may benefit also.

This shone through like a beacon to me, first because I had similar experiences of tra umatic life events and, second because I had a similar epiphany, at University. Unless you also go through a genuine transformation, I don't believe anyone can appreciate the magnitude of effect this has on every aspect of your being. Mine did not just change how I see the world, but also how I exist within the world, forever! I had just began my undergraduate studies (BSc Hons Complementary Medicine and Health Sciences) and been introduced to homeoepathic philosophy (which managed to explain all of my previous 'illness issues' where conventional models had nothing to offer) when my beloved cat was diagnosed with bladder cancer and the advice from her Vet was she be 'put to sleep'. Taking my 'new' philosophy into account, I wanted to see if anything could be done to help her re-balance. As the owner, I 'took the case' 
as I had been taught and with my tutor worked out what the indicated remedies would be, if I wished to administer them. At this point we had nothing to lose and everything to gain.

I could not have been more amazed when first her (my cat's) distress eased and then the tumour (which the Vet had shown me how to examine, so I could determine the optimal point of any 'intervention') began to change shape and 'feel' smaller, this accompanied by her passing small clumps of 'vascular cells' in her urine. The philosophy 'proven in action', I was a firm convert, which meant turning everything I had ever learned or understood about health and illness (through dominant discourse and my original therapy training) on its head. This was my epiphany. As I learned more about homoeopathy and vitalistic philosophies, I became more convinced that mainstream discourse was looking at everything 'the wrong way' (surrounded by a full cohort of similar thinkers), also becoming quite the conspiracy theorist, seeing the only 'winners' in conventional medicine as the large pharmaceutical companies and the medics they pay to administer their wares. This conviction has mellowed over time, however remains fundamental to my journey going forwards since then.

Like Veronika's, this has led to an intellectual isolation that in many ways is worse than a physical one. People may or may not be around you, but being unable to connect with them intellectually, spiritually and emotionally, can create a chasm of distance. Veronika shared with me casually one day that neither her family nor friends agree with her 'new' philosophy since the Camino (a region of Spain used as a Christian pilgrimage), even thinking she is a 'bit weird', and this has left her feeling very isolated.
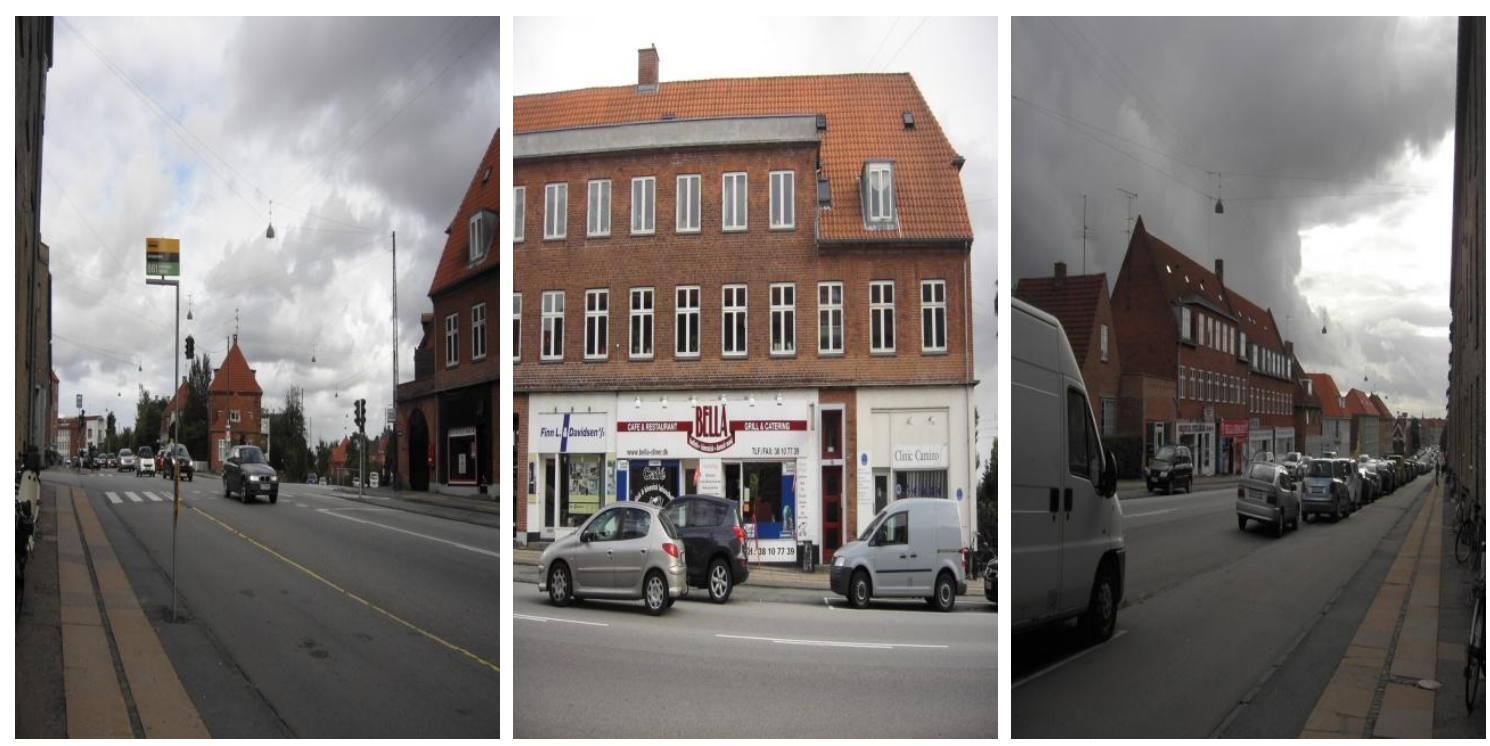

Triptych 9.5: The exterior at Clinic 2 
To combat this, she tries to be pro-active in how she stages her therapy space, creating potential to 'bridge the gap' by letting people see some of the events and their impact, so they may know her and connect with her better. This seems to work too, as clients do read the space as a part not just of their therapy but of the therapist who delivers it. It may not always be easy to fully understand the inclusion of different items or aspects, but this in itself makes them speak a language that invites conversation and connection. Having such artefacts around also helps Veronika to feel grounded and connected to this place, and supported in both her acts and convictions. This is not so much a case of distraction, as it is invitation to be.

I think this is especially important in CAM, as therapists (and researchers?) may operate in a world with alien views to their own and, without forging successful connections, can lead to inverted over-zealousness, disassociation from all around (me) and depression. When this happens, retaining a 'healthy' connection to inner beliefs can be difficult and for me certainly (as I have already said) this has permeated into my research practice, although curiously not my clinical one, contaminating both my thinking and my doing. Veronika (like Theo in Chapter 8) seems to recognise this, through her experience of returning to Denmark after visiting the Camino. She found the conflict between worlds unbearable and chose to quit one in order to live within the other. Her 'home' is now a physically lonely one, but spiritually rich.
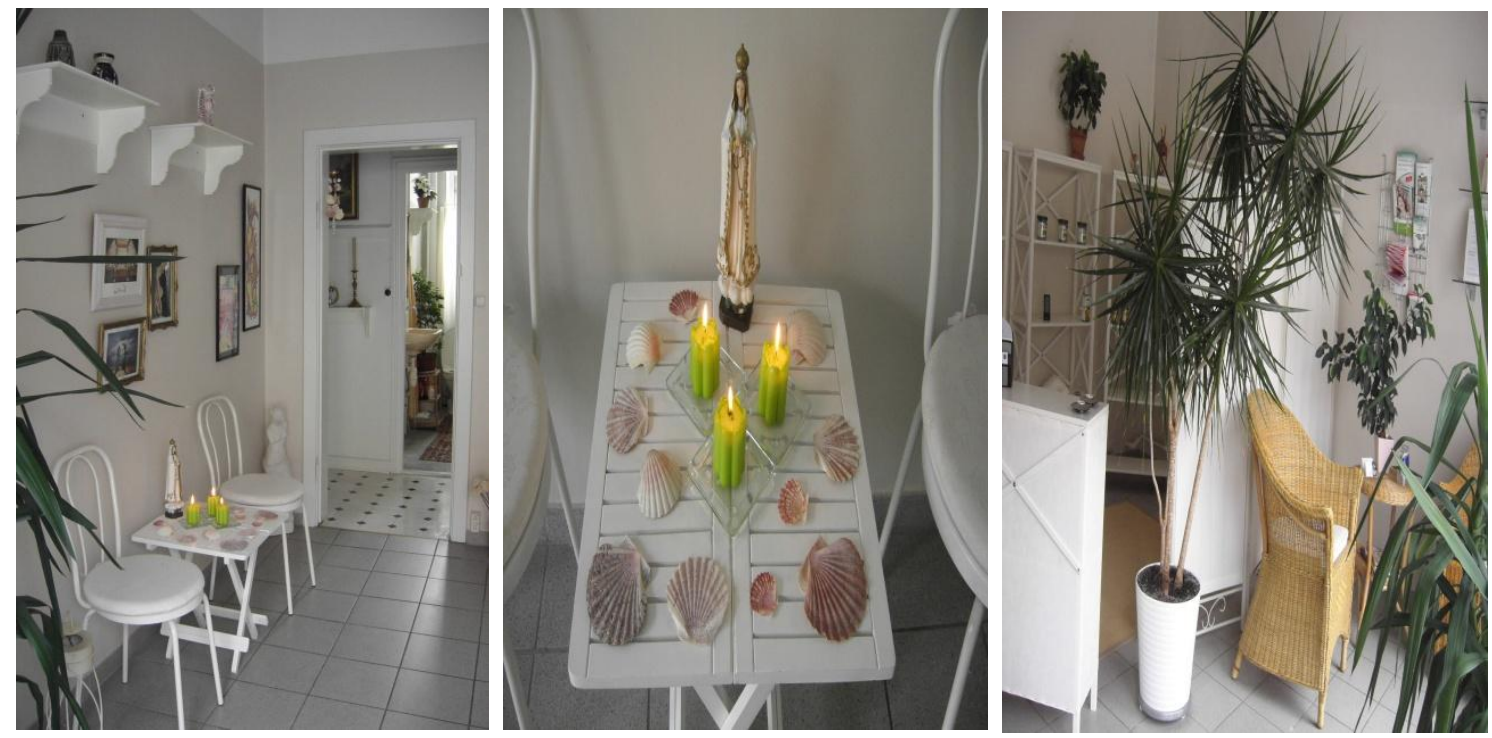

Triptych 9.6: The entrance and waiting spaces at Clinic 2

The 'space' Veronika has created for herself (in her home and clinic) since then is full of reminders about what is important in her life and her work, from the collection of shells she brought home from Spain (Camino) to the statues, altars and artworks she has created to express her connection to self and spirit. In essence, it may not be important whether these 
have any direct impact on clients (except as she mentioned about 'her image' and an invitation to engage in dialogue with her) as they do have a direct impact on Veronika. Without this she would again feel lost and conflicted in an alien world of everyday life, function and fashion.

For Veronika, it made a nice change to have someone like me around, who she could genuinely dialogue with on a shared level (outside her former cranio-sacral tutor). This was a different relationship dynamic than I observed with clients, where dialogue was often more about Veronika sharing the benefits of her experience and 'teaching' a different way of being. I appreciated this too, as it gave me an alternate relationship to my usual ones (in practice and in University) where I am either teaching or defending my perspective. Indeed, as a researcher, I found being around people who share my level of spiritual interconnectedness gave me similar succour to that Veronika gets from having her Camino shells, statues and artworks around her, a supportive 'space'. This may explain why I have, at times, found it so difficult to be back within the real world, after my data travels (like Veronika did after her Camino travels) and why Veronika, absent of much direct human support, feels more settled in her space than does Josefine, with a filter through this in her engagement and outcomes in practice.

\subsection{Act 2, Scene 4: Making better, making friends}

Completing the picture here, I want to illustrate my observations with Veronika from a client perspective. Eva (not real name) is one of Veronika's clients whom I observed receiving zoneterapi (reflexology) and interviewed afterwards. New only since opening this clinic (did not follow Veronika here) Eva exemplifies the positive relationship built with Veronika and the role she assigns this whole presentation in creating and supporting this potential.

Veronika and I were sat in the little kitchen at the rear of her 'shop' when the bell on the front door chimed, signalling that someone had opened the door and entered from the street. I waited and watched from the inner hall as Veronika, casually dressed in brown cropped trousers and matching t-shirt, went to greet Eva and then followed as they passed through the hall into the therapy room at the back of the shop. A clean space painted in natural shades, I found this cooler than the treatment area at Clinic 1, perhaps because of the high ceilings and crystal chandeliers that provide additional light. Feeling 'stark' in comparison to the entrance space, this was clearly staged for treatment, housing both a therapy chair (for reflexology) and traditional therapy couch (for cranio-sacral therapy and Reiki), whereas the entrance was staged for welcome. Eva was quite heavily pregnant, but managed to get onto the therapy chair unaided, eager for her 'much needed' treatment. It had been a 'long' day! 
The sun streamed in through the small window, but there was still a chill in the air, so Eva pulled up the warm blanket Veronika had left out for her up, over her tummy and leaned back to relax into the session. I sat on the large wicker chair just inside the door and watched as Veronika began to first loosen up Eva's feet and ankles with pressure points and gentle mobilisations before applying cream from a tub on the portable trolley between the two therapy stations. An underscore of 'social chatter' accompanied the first half of the treatment (approx. 30 minutes) before tailing off into a peaceful silence, during which Eva closed her eyes. Throughout both early and latter stages, Veronika worked from left foot to right foot, then left foot to right foot in a fluid method that I found different to the model I had previo usly observed in UK, and with Josefine, where each foot was instead fully completed before moving onto the other. Specific moves caused Eva to 'twitch' in a similar fashion to Isa, when she got Bowtech from Josefine; similar but less intense.

Veronika continued to work both feet, switching from left to right, left to right. I noticed that her fingers and thumbs were always spread out, never together as I have seen with others. At times too, Veronika paused 'trans-like', and I wondered if she was also adding Reiki into the mix. For the last 10 minutes of the session it was clear that Eva was sleeping and breathing deeply, undisturbed by the movement above our heads (in the apartment building). She was totally relaxed. At the end of the treatment, Veronika wiped any remaining cream from Eva's feet at which point Eva began to stir. By the time Veronika returned from washing her hands, Eva was sitting and ready to arrange her next appointment.

Eva and I stayed in the therapy room together, after the treatment, as she had agreed to also participate in an interview. Veronika (who's apartment is directly across the street) suggested she go home until we finish, returning to lock up for the evening. Alone, in the silence, I got Eva's perspective on the events I had witnessed and her general experience here.

Eva's narrative:

I have been a client with her [therapist] for six years now. I come for zoneterapi around two to three times a month, and she manages my lower back pain and fluid in my legs. I used to get therapy from a place in the City, and was quite satisfied with that, but then she [therapist] opened up here, and it was close to where I lived, so I decided to try it. I immediately got a much better connection with her [therapist] than where I went before, and although the treatment was similar, the experience was so different that it was an easy choice to move. Here, I get a continuity of care that I never got before, our conversations are meaningful, and she knows me, she knows my life. This 
treatment is all about me. I am active in it, not passive like before. In the previous clinic, I had no relations hip with the therapists, we never connected, and they were just someone who delivered a treatment. Here, because we have a relationship, she [therapist] is very much part of the treatment, and she knows just where to press!

This place has a peace and tranquillity that the other place did not have. I don't think it's about the location, although that was in the City and this is not. It's more that there is just one therapist here instead of lots, which makes the centre less busy, and means I am more memorable to the therapist. I like the private therapy room. It's much better than the other place, where there were curtain cabins, and you could see and hear the other people. I wouldn't go for therapy in a communal room now, and think that place has a role separate from the treatment. It is clear that she [therapist] feels comfortable here, and she mediates this setting into the experience. She makes the things around her work. I live around 20 minutes away and come by car. I am often stressed when I get here, due to the journey, and am often late, or nearly late! I like to get the last appointment of the day. That way I don't worry so much if I get held up. This melts away once the door is closed, and I am inside. She puts me in a totally different place.

Like previous reports Eva places great importance on the therapist, in this case Veronika, as a vital part of the whole treatment, mediating the independent values of this setting and the treatment into a single event and giving her a more effective experience as a result. Bound in this 'whole relationship', mechanical aspects of therapy are enhanced ("she knows just where to press") as are their emotional connections ("she knows me, she knows my life"). The critical aspect here though, more than the 'strength' displayed by Josefine that was noted by Isa, is the 'depth' of relationship these characters share.

Veronika is not regarded by Eva as "just someone who delivered a treatment". They share a connection, even a friendship, where Veronika opens herself and her skills to help Eva. This lends a different quality to these interactions than where Eva previously went for therapy. There, there was both an absence of peace and tranquillity and an absence of continuity and lots of therapists saw lots of people, screened only by curtains. The result was that Eva never developed any positive relationships which limited the potential of her therapy and outcomes. Here, by contrast, Eva finds that she and Veronika immediately had a better connection, whether this was enabled by her individual/personal approach, the conversations they shared, her selections of art and artefacts (something that Veronika has concerns about, but stages 
here anyway) or the desperate desire Veronika has to feel connected herself in a reaction to the model of isolation that otherwise dominates her practice.

This raises an additional issue, raised by Veronika (in Act 4, Scene 3 ) about how those who respond to 'her', respond best to the treatment and those who do not connect with 'her', respond less well. I include this here rather than where Veronika raised it as I did not realise its full significance until I placed it next to Eva's story. The issue of a 'responder versus nonresponder' in this context is something that I observed widely, finding trust and compliance key indicators of good outcomes. This was more than 'bedside manner' or 'empathy in action', but a dynamic level of connection where the client/patient chooses to receive what the therapist gives. With the incorporation of Reiki within all treatments, this is a multi-level engagement that I found as powerful as Josefine's zoning or Theo's (chapter 8) self-belief, and present also in Simon's (chapter 6) reputation and Emil's (chapter 7) vitalism. Lacking any real clarity within current research/clinical findings, comments by Eva seem to suggest that 'connection' is at the heart of this, for the therapist with their application, supported by a personal conviction and enhanced by the surroundings.
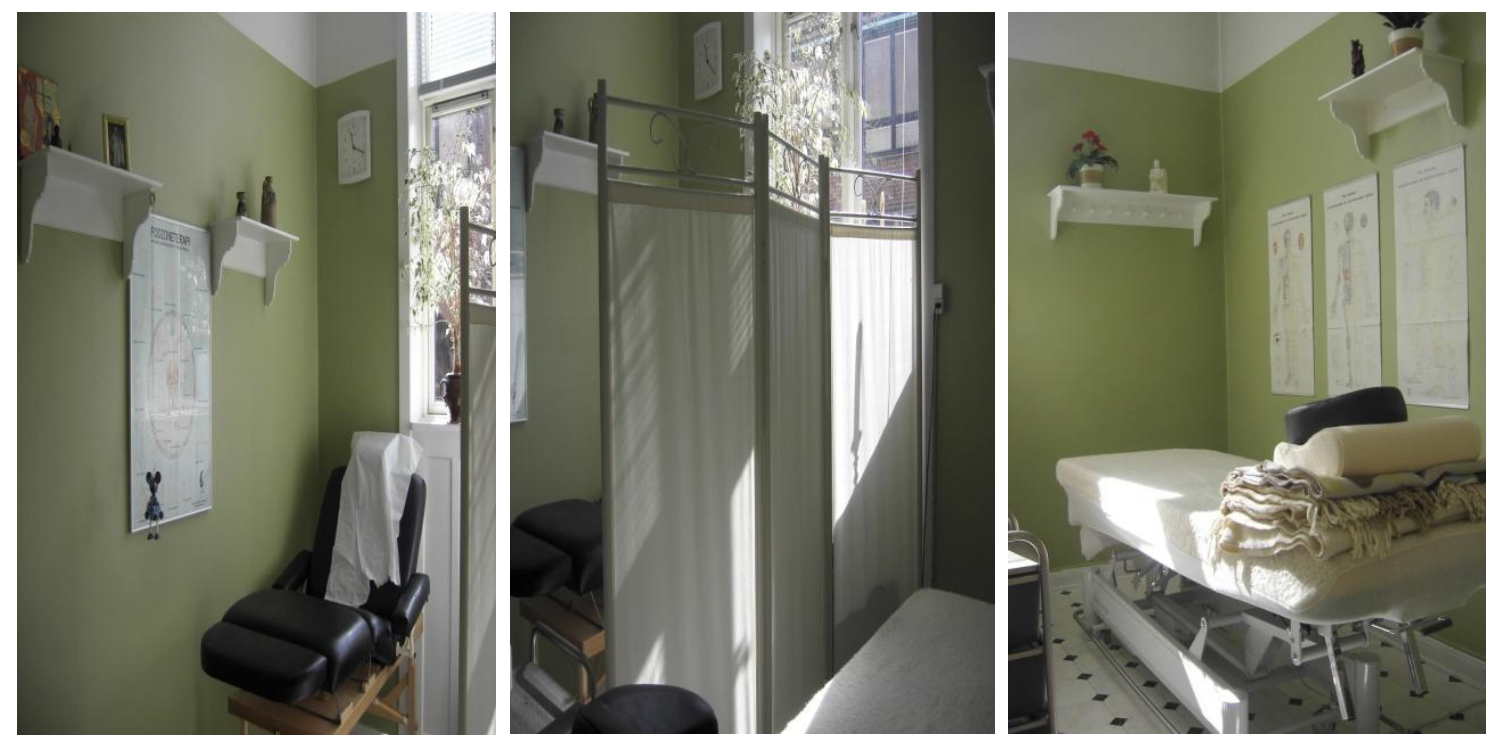

Triptych 9.7: Therapy space at Clinic 2

I did not observe Eva with any of the nameless therapists from her previous clinic, however I did find a closeness between her and Veronika, a more candid relationship where Eva was relaxed and able to be open and honest that may have been the link for her between this 'space' and better therapy. This is something that I have also found across the study and recognise from my own practice (in therapy and research). Good connections, however they 
are made, nearly always result in better outcomes, either because this creates more caring or more openness which is a 'space' itself, where there is potential for more target ed action.

\subsection{Act 4, Scene 5: Critiquing the whole (holistic) therapy performance}

I have referred to this lived production as a 'tale of two clinics' although these were actually just two examples from the many similar clinics that (over) populate this City and its' districts. State sanctioning, recognition and funding here means zoneterapi (reflexology) has become a popular intervention in Denmark, both to access and train in, therefore becoming the first line choice for many CAM therapists and for clients, even if sometimes this is not the most appropriate one to always select. In a spirit of sustainability, the clinics I engaged with also provided additional therapies, delivered by the same therapist, to enhance models of care and improve income opportunities and while similar in structure (lock-up shop staffed by a solo therapist) the motivations for each practice are guided by different ethos's, meaning their practices are quite different to observe and experience.

As much a display of the values and beliefs of the practitioners as they are their freedoms of expression, Clinic 1 brings additional elements to distract clients from their environment during treatment, such as music and candles while at Clinic 2, additional elements have the different emphasis of connecting/supporting the therapist with reminders of her epiphany and thus support her engagement with this work. The result is like two faces of the same coin, one intending to be primarily functional, and delivers this, and one which first meets and then exceeds this aim to deliver an experience that is both functional and spiritual. Their differing 'success' in practice then, is perhaps more a result of the individual skills and experiences of these therapists in mediating their practice and settings, to assist connections with themselves and their clients.

At the time I was there, I noted that each of these therapists 'feels at home in their work' and that this lets them 'be' who they are and clients to 'see' who they are. In 'the moment', I felt strongly that this played an integral role in their ensuing relationships and that a therapist who works in a place where they can be themselves and 'connect' with themselves is better able to develop good, effective therapeutic alliances and outcomes. This suggests that building successful therapeutic relationships is not simply a matter of active choice, but that the conditions also have to facilitate or support this - by creating potential. Having since reflected on this, and other issues raised by the data, I still feel that to be the case. 
Making good relationships (and friendships) here, I found these characters to be a joy to work with and as central to my experience as their clients reported them to theirs. Equally I found them lonely characters, with a fragility that came from their individual contexts and impacted their unique performances. For Josefine, the pressure appeared primarily financial, creating a reality she did not want to (or know how to) face while for Veronika, this was more intellectual, as she tried to bridge the distance between herself and those around her. These tensions seemed unbeknown to clients, however, through the discreet strategies each employs, and great satisfaction was reported regarding these different therapists and therapies, finding their combinations inextricably linked.

\subsection{Act 4, Scene 6: Reflecting on my research performance}

Engaging with this final presentation of therapy, I found no less excitement than the first time around. In some ways, I also found my work more satisfying here as it signified the completion of a task many in my school felt beyond a PhD study.

Perhaps here, more than anywhere, I began to see how knowing who you are and being who you are can aid both success and fulfilment in life, therapy and research. Across all sites the value of individual journeys and healing stories had been evident but here, gaining the last ones, I truly understood this. Maybe this was because, in their solo performances, I had seen the closest resemblance to my own practice or maybe it was that I could imagine how these characters may fair or feel in others' environments or others would feel here? This was only my interpretation, but here again I saw the unique fragility of characters who truly feel unsupported in their work, either because they have no one around to shoulder the responsibility with them or because they feel ill-understood and alone in the world.

This chimed with me deeply although I don't think I really recognised its full impact at the time. My natural reaction whenever I have been challenged like this has always been to build my defences even higher than before so that others may never know how vulnerable I really am. I don't think I did this while still on site, but I certainly did upon my arrival home when I began re-listening to the live data and noting my responses. As with the other sites I had sought to pick out the parts that resonated most with me (in line with my methodology) but, for a long time, failed to acknowledge the personal impact of this method on me (I had been more concerned with exploring my personal impact on the data). The characters at this site, more than any others, brought out sentiments in me that I find hard to bear; the lone responsibility, feeling ill-understood and dissociation from those 'at home' to the point of depression. Seeing these here, in front of me, I began to feel uncomfortable in myself and this made it harder to 
be open and honest, exposing and exploring how I felt about different aspects of this whole experience (personally and professionally). This feeling lasted through into my early writing too, the degree of impact depending upon whether I was in a place of good relationships and good connections or abandoned and lost. In my darkest depths I could not see this, of course.

Yet, this was to prove my greatest lesson and, as I look back now across the entire study, I can see how this has been a journey of discovering not only what a CAM practitioner (me) can reveal about the nature of CAM therapy practice to aid CAM research, but also what research into CAM therapy practice can reveal about the nature of CAM practitioners, to aid (my) healing. This exposure of therapy performance has made me face the truth of my role and reactions. Like Josefine and Veronika, I also hold insecurities due to fragility, isolation and lack of support. Seeking succour beyond connection with patients was equally mine and these characters reasons for participation, but seeing this played out before me had the response of preventing me from connecting with and communicating their stories in any truly meaningful way: until I finally gave myself the 'space' to realise this. This then proved a catalyst to help better target my whole performance, which significantly improved both my potential and my outcomes across the whole study. 


\section{Chapter 10 - Finale Ultimo}

A number of themes emerge from the many complex 'threads' of this performance. Some of these relate to therapy practice and others to the practice of research. Applying the concepts of holism and theatre, the distinct episodes of the UK, Holland, Cyprus and Denmark create a larger, interconnected whole of findings and responses that speak of more than the places and performances I witnessed. They expose and explore the nature of therapy and of researching therapy, which are two concepts that I find inextricably linked.

This is not just a matter of discovering whatever the researchers gaze can capture, but also the framing that directs whatever the researcher observes and the frame which creates the potential for individuals to conceive this.

The question my study asked at the start was, "What can a CAM practitioner's perspective of studying CAM practice teach CAM researchers about researching CAM?" I will now seek to answer this. Before I do, however, I want to explicate my process, as it is the process that has given rise to the product.

\subsection{Reconceptualising CAM research}

Throughout this whole study I have observed many people and listened to many stories. Each has shaped both the process and the product of this work. Set within a prevailing discourse where practice is not readily accepted as a source of experiential knowledge (Mulhall, 2001), I set out to explore and understand the performance and experience of therapy from a holistic perspective, and apply the very philosophy that underpins many of these modalities to their study.

An ambitious project, my earnest attempt to 'see' therapies holistically began by recognising the potential for a vital materiality in their presentation, something that many of the underpinning CAM theories allude to and even base their practices on. To achieve this, I both developed and applied a novel, holistic research approach, to perceive, capture and explore the multi-dimensional nature of complex lived experiences, as they are performed in daily practice.

I decided to engage 'in the moment' and discover how holistic philosophy and CAM research may become better integrated. This involved recognising my own strengths and building a study approach around these. I engaged a holistic frame to guide every aspect and ensure a 
paradigm fit both with my own personal philoso phy (I regard myself a holistic thinker) and that of my topic area. Developing this idea, from the initial selection of a research question through the design, execution, analytical and performance stages into a coherent production has been a significant personal as well as professional challenge. In this I have sought to enact both a method and means of capture and dramatisation, for the reader, so the holistic picture of therapies I witnessed may be critiqued and shared to reveal those aspects of therapy (and research) that standard approaches overlook. Within this, I have also trialled a novel methodology, developed for the purpose, which calls upon the researcher to 'suspend disbelief' and enter an alternate reality and view, where the performance and experiences there can be captured and shared as the characters do. Much as a theatre critic writes their review after the curtain has fallen, critique here also comes later, when disbelief is re-engaged in a 'space' where both the meanings behind the performances observed can be questioned, and also any claims of knowledge arising from these.

Lees (2013) refers to the 'practitioner-research' design as the "flexible design" model described by Robson (2002:5) where the design/approach evolves as the research proceeds mirroring a therapeutic model. I take the metaphor but view 'holistic practitioner-research' as something more active; an intentionality to seek understanding through synthesis and interconnectedness driving evolution in the approach. As a different practitioner to Lees (2013), it is natural that my practitioner-research approach may differ also, to reflect the unique nature of my different training, philosophy and practice. While adapting and evolving through the process, my application has also been naturally different for 2 main reasons: I am an inherently different practitioner; and my patient here was not a human case-study with whom I simultaneously performed a therapeutic role. Rather I have been concerned with the potential to examine CAM practice, as holistic phenomena, as a precursor to better effectiveness/evaluation research.

Acknowledging and utilising my natural position as a holistic thinker, I first recognised how the holistic paradigm influences my every thought and action, guiding my epistemology and ontology (whether this is comfortable or not). Not a practice or method itself, this is rather the mode by which I dramatise how I 'see the world', and in the choices I make with methods. Such is my commitment to paradigm fit, that when no established or 'off-the-peg' methodology or method seemed to offer the degree of holistic perspective I craved, I was compelled to create my own. As part of the overall plot enacted in this production, my experiences as a therapeutic and theatre practitioner are implicit in this enactment and, 
though novel, I felt this would generate greater validity and reliability than studies where paradigm fit is either compromised or unacknowledged.

Already aware that part of the 'magic in therapy' comes from the interconnection between components of practitioner, purpose and place, I proposed Theatricality as an appropriate methodology and method to meet the study aims. The parallel drawn between therapy and theatre (such as casting of practitioners with unique biographies and training paths, the range of modalities offered with the nature of their employ, and the physical and dynamic compositions of space and place) not only creates unique insight into the role and value of performative or contextual variables, but provides an accessible option for perceiving and capturing the complexity of these performances, holistically. Layering each aspect, function and purpose of the production, the parts and the whole are viewed simultaneously providing both the potential for results to be better contextualised and a level of meaning to be created that is capable of resonating universally with practitioners and patients. Underpinned by the support of heuristic research, and side-by-side with ethnography, this novel approach has enabled an exploration of this issue in a way both congruent with the philosophy that directs many CAM modalities, and directs me!

Finding a natural place within this, the associated Theatricality framework method gave the means by which descriptive scenographic data could be holistically gathered, careful to perceive and document each aspect and component of the staging and its role in these performances, which synthesised via performative criticism with ethnographic observations and semi-structured interviews, produced a uniquely holistic research approach, of potential use within any performative arena. Moreover, it has proved capable in generating comparable data from both a volume and range of diverse settings and participants, making the potential of Theatricality as a whole research approach an exciting issue. Within this study, it has certainly allowed me to both uncover the means to answer, as well as the actual answer, to my research question demonstrating that holistic perspectives can be as beneficial to research as they are within therapy, making the case for the further development as a research tool.

Of course, problems arose within the research process but, here again, Theatricality proved a useful tool, mirroring my research performance back to me until I could perceive where any issue or blocks lay. Often, these turned out to be the problems faced by holistic (CAM) practice every day, whereby limiting or constraining forces of reductionist culture and fragmentary perception, both within and outside the actors context, caused unintentional modifications in an otherwise authentic delivery (at least until these are noted and reversed). I 
myself, influenced many times by such perspectives, found attempts to present or write this thesis authentically (a marriage between holistic ideals and academic requirements) a difficult challenge not faced in the performances of design, data capture or synthesis. Perhaps due to the novelty of this approach, or my own inexperience of writing it (the doing is an everyday natural state for me), achieving this final product has proved a long and challenging journey.

Within the interpretive and constructivist phenomenological paradigm, where multiple truths exist for each observer, my method has tried to connect the audience response to the performance that generated it and present this as an interactive experience more closely reflecting the moment of data capture, before the experience is fragmented and distorted beyond all recognition (i.e. traditional or positivist approach). The data I have portrayed and my own synthesis of this is as honest a critique as I can present to illuminate the nature of these complex and nuanced phenomena, as I experienced them to be, revealing many aspects that more popular research methods overlook.

Through a constant reflective engagement I now argue that CAM therapies should be studied without reduction or 'simplification', having also proved this to be achievable and leaving the way open for researchers to ask, and answer, less restrictive research questions. I did not simplify these performances, as academia often would, because I wanted to see whatever was hidden, without compromise. At times this was a hard challenge for me, and I'm sure would be even more challenging for anyone firmly held within reductionist thinking or a fragmentary culture club (i.e. academia or biomedical discourse). However, my study demonstrates that if the conditions (or context) are supportive enough, then anything can be (at least) possible.

Theatricality methodology, enacted through this study, may or may not be the panacea for research I first thought it could be. However, its use here has enabled me to step inside my own performance and those of the clinics and characters who participated, and examine the ways each aspect connects to the whole. For me, it has served a purpose, and it may for others too. Certainly the range of contextual variables present within real-world CAM therapy is diverse, as members from both the Whole Systems Research and CAMbrella groups have shown in recent years. One approach to this is to deny the value of diversity, apply large clinical trials and population studies and eliminate the need to deal with diversity - but then practitioners and patients tell us such studies lack model validity. Another approach has been to study aspects of diversity in isolation from the whole, but often these samples are too small for findings to be generalised and only tell us about 'those aspects' anyway. The third way is one that no-one has yet attempted, although I suspect many would like to see, and that is a 
large scale study of CAM that captures and explores the diversity, micro-enough to gain real depth of meaning but macro-enough to be transferable to the wider practice and research communities. My efforts here, evidencing the possibility of capturing complex and diverse study data (which professors in my school said couldn't be done), represents a small but significant step towards this third way.

In viewing the complexity of CAM therapies as a gift, not a burden, I have enjoyed many years of clinical engagement in these. I have now applied this same perspective to their research and created the potential for a quality of data that has so far been overlooked within CAM research, to be revealed. While personal to me, I believe this experience raises both potential and questions that any researcher should ask themselves in terms of what they are looking for and what they are seeing when they study complex phenomena?

\subsection{Comparing complementary health centres in the UK and Europe}

At the outset, this study asserted that 'space' creates the potential for relationships and that, in order to understand the full performance and impact of CAMs (as a pre-curser to valid clinical trials), it may be necessary to also expose and explore the nature of CAM 'spaces'. It was never my intention to measure the effects of different environments on therapy.

To do this, I chose to enter and observe real-world practice in working clinics, speaking with real practitioners and patients - not in a sanitised setting, removed from their usual place of care - but within those settings, capturing not just a 'tell' but a 'show and tell' of their experiences. I then set those findings against my own experiences, both in each centre and outside; my own back-story providing the rationale for any conclusions.

The principal aims I enacted were to capture and explore the whole, complex nature of therapy delivery and experience across a diverse therapeutic and geographical sample, utilising a holistic frame and perspective. I wanted to see whether this may uncover aspects of the modalities, the practitioners or the delivering context that other approaches overlook yet hold value for therapy and to those whom encounter these. I decided to put myself inside the study, and the data, to expose and explore the power of these connections and embody the experiential knowledge contained within each performance. This, I hoped, may reveal a solution to the theory-practice gap I had observed.

As components in a single research narrative, the ' 4 Acts' each play their own significant part, relative to the others and together build into a larger performance of CAM therapies in different spaces. This has revealed much about the nuances of practice, typically overlooked 
within research study, and yet so implicit to everyday lived experiences for the practitioners who deliver them and the patients who receive them. One of the few common observations, across the sample, was how committed these practitioners are to their 'arts'. It is a true caring vocation (not a career) that drives and sustains them, and this is evident from their performances. A living example of different cultural norms, the staging and delivery of practice in each centre was as holistic as the prevailing position would permit practitioners to be. In this sense, both the aims and actual therapies were contextually dependent variables.

Beginning in the UK, I found a presentation of therapy that seemed to almost mimic the clinical model of the National Health Service. Familiar, friendly and functional, therapists here offer treatments based on a primarily biomedical understanding of health, where holism means acknowledging that people are a whole of mind/bo dy/spirit, but without any real conception of how this can impact health or should impact clinical care. For patients too, who are often used to an 'even more' reductionist model of care, this provision was more than adequate; they seemed to accept that if they wanted 'more' than resolution of physical pain (with a genuine cheery smile) they should go elsewhere.

The practitioners here seemed to me to exhibit a conflict between wanting to be different yet also afraid to look different - or risk rejection by the dominant discourse. This results in therapies sometimes presenting a diluted model (such as exhibited by Simon) which carries a potential impact for patient outcomes. The capacity for awareness in appreciating impact from this is both uniquely dependent and highly variable (I noted clear differences between myself and between the different characters here).

In Holland, I found no such conflict, as the characters (practitioners and patients) all seemed to share a common underpinning of philosophy in anthroposophy. Playing an active and visible role in each performance, this practically compels all practitioners to offer a service that aims not to relieve or resolve only physiological complaints but to heal, by making people whole again, within not just their immediate physiological environment but also their spiritual, social and ecological ones. Therapies are accessed as part of GP care and are applied to stimulate movement, development and progression on a dynamic, whole person level, where the spirit is moved in action, within which the potential for healing can occur. Delivered within a supporting culture, many of the patients here embrace the principles of anthroposophy in their wider social lives too, and so seek this care to supplement their education and lifestyles. 
Holistic here enjoys a truer, fuller meaning related to wholeness and 'being at one' with the world, achieved through interconnections with the self, with each other and with the environment (social and ecological). Directed by this discourse, all modalities, medicaments, staging and human interactions are composed and delivered with intent of such effect and affects. Within these truly therapeutic relationships, patients often spoke of feeling 'carried' by their practitioners, and therapies, until they 'could walk' (or were well) again. In both their complexity and composition, this 'space' was physical, emotional and spiritual. Therapy therefore seemed to go 'beyond function' to deliver enhanced connections with the self and wider environments; essential for this 'holistic' healing.

In Cyprus, I met a unique and single minded 'guru' who appeared to be set in direct opposition to the dominant biomedical discourse, giving him a freedom and power to enact his own unique approach to complete patient care. A product of his own healing journey, he has discovered that using multiple modalities can simultaneously affect the multiple levels of human health and existence (spiritual, psycho-emotional, physical, energetic, environmental, social), and so directs all aspects of this production and performance here.

Similar to the UK (where this practitioner was born and raised, albeit to Cypriot parents), holistic here is taken to mean all levels of the human in a single entity but recognises also the inter-relationships beyond this, in the geographical, ecological and social environment. The impact of this on a patient group who often arrive with serious health complaints and zeroprognosis is feeling 'born again' and 'resurrected'. This level of thought and intention may be beyond the awareness of many patients here, however any impact is clearly observable both for them and in them.

In Denmark, I learned that therapy enjoys a better public image and state-endorsement, so is often accessed equally as a preventative measure and in response to direct events. Therapeutic applications link to these different motivations with delivery at Site 1 providing largely biomedical or physiological outcomes, while at Site 2 the intention is more about spirituality and lifestyle. Similar to the UK, personal connections and unique skills seemed to matter the most, although there was also clear evidence of trying to make the experiences pleasant, with benefits that equally affect the practitioners and the patients.

Holism, where applied, is la rgely limited to the conception of a total, single entity in the human body, mind and spirit. This bears more congruence with the UK interpretation than either Holland or Cyprus. A critical difference here was that, despite providing a combination of 
therapies, the dominant model was of single practitioner clinics, making providing therapy an isolating experience (for the therapist). This gave a greater potency to the quality of therapeutic relationships, being often reciprocal in nature.

From the staging of this therapeutic set to the performances within it, each of these productions brings a message about the intent and potential of therapy within, that impacts the experiences of each performance, where nuances in the presentation and value of each component of practice (character plot and set) are known to local participants but unknown to others. Similarly, local participants do not appear to know how the practice of others may compare with their own, enforcing each production into a unique therapeutic characterisation, where the primary reference frames become the isolated paradigms, philosophies or discourses of these individual characters, plot or setting.

Comparing these 'others', it is possible to perceive each practice-specific performance with 'new eyes', making the familiar unfamiliar, so each can be critiqued with a different honesty and reflect on the treasures within. In each scenario the prevailing culture and paradigm interactions are dramatised, with those of the modalities and their practitioners enabling, encouraging or limiting the mode and potential of therapy application and individual outcomes. Alongside place and space, here selected, constructed or composed by, with the compliance of, or for individual characters these aspects of practice appear more integral to practitioner performance than previously documented and as such warrants further exploration.

There is impact for researchers in this too, as during the process of engaging these different performances my performance became similar infected. I was exposed, as patients are, to the different intent and ethos dramatised by these characters, plot and setting and this affected how I also engaged there, impacting my potential for relationships with these places and the data they revealed. The full implication of this really only became explicit for me during my writing up, as 'back in the moment' I often found my style and quality of writing to reflect the functional; uplifting; esoteric or pragmatic natures of the places, people and data I was again engaged with. This was unexpected for me, as research is meant to be a detached activity, and my experience was anything but detached. In terms of my final performance (this thesis) such interpersonal engagement was not a peripheral aspect, but an integral one, and learning how to step back into my own 'space' to identify this with clarity has been a hard act to deliver. 
One thing I did observe, across this whole sample, is that the heterogeneity within clearly suggests 'one size does not fit all'. With this heterogeneity, and many issues as yet undiscovered, the popular practice of controlling variables within therapeutic performance seems at best premature, particularly as such variables are often regarded as 'placebos' (fake) by those outside these experiences. Indeed, practitioners and patients across all sites found additions that were 'pleasing to the soul' had both profound and efficacious abilities, when evaluated in relation to the whole production and performance.

Across all of the sites, there was also a strong suggestion that when both the practitioner and place are bound with each other, either in presentation or interpretation, these connect to influence therapy experiences and potential outcomes. When practitioners put care into the energies of the site, this is enhanced further with patients becoming sensitive to those; similarly, when practitioners do not take care of the energies of the site patients do not become sensitive. Appearing to 'follow the lead' of practitioners, I found patients will also view the various aspects of their experience as fragmented if their practitioners disconnect these. While specific to certain settings, this is a shared issue across the sample. Unpicking such elements would, therefore, appear both impossible and undesirable if, as it appears, the synergy of the whole between people, plot and place is what really matters.

Just as the design and layout of a therapeutic space can be a facilitating or constraining factor, so can the design and layout of a thera peutic/research frame. If those delivering and receiving these interventions do not view them as a homogenous group then there is invalidity in academics, researchers and policy makers presenting them as such. Appreciating the unique differences between modalities as gifts to be cherished, not a problem to be denied, can only allow more honest and critical appraisal of their benefits and limitations to be delivered.

Set against one of my first experiences as a CAM researcher in Berlin, where I witnessed academics and physicians arguing about the most suitable umbrella term for these therapies, I have found that there is also no 'one size' for engaging the broad field of CAM and that research has to be more open minded to the local perspectives (also a finding from CAMbrella). Within the UK, the term CAM did hold some understanding for participants but without any active meaning or reference to specific philosophies. In other European settings CAM, as a term, seemed to hold little or no meaning at all. Characters in Holland instead preferred to use individual modality names; Cyprus participants liked the terms alternative or natural therapies; and in Denmark alternative was used, but as a means of distinguishing these 
from mainstream healthcare (it did not signify an alternative philosophy). This is a matter for researchers and academics to engage with better.

As my own story testifies, however, simply intending to be or act a certain way does not guarantee this will happen - the conditions or 'space' also have to be right, to support this. Read's (2005) theatrical and architectural conception of 'space' as creating potential for relationships seems to carry into this arena too, offering a way to perceive how the different presentations and staging relate to the performances therein. I find this to be as true for therapy as it is for my research into therapy.

Until this present study, the similarities or differences within practice performance have never been revealed on this scale, let alone critiqued, bringing a deeper level of knowledge and opportunity for reflective practice. Indeed, these findings have real power, particularly given the size of the sample: comprising the views and experiences of 31 practitioners and 47 patients within 5 clinics across 4 countries. Whether contextual variables manifest in either more or less rich therapeutic performances, such dramatisation influences the experiences of therapy for all.

It is clear that research into these approaches needs to be more aware of the complex realities within local practices if their findings are to have either congruence with those populations they aim to serve (assuming this is actually the practitioners and patients, not just other academics). Ritenbaugh et al (2010) have noted how many current researchers in CAM can struggle to identify with the nature of therapies, due to either the selection of methods applied or because the researchers are not adequately equipped to capture them. More research of this type could go some way to remedying this.

\subsection{Conclusion}

This study did not set out to determine the effects/effectiveness of different contextual variables on patient outcomes. Rather it was to see what a 'CAM practitioners view' of CAM practice could reveal, to benefit CAM research. To do this I developed a critical-subjective frame that would both let me into different CAM performances and also provide a template for engaging with the whole staging, composition and 'acts' therein.

A challenging task to enact, I learned that CAM practices and practitioners appear bound by their context and space, creating or limiting the potential for relationships with their therapies, and with their patients. I also learned that CAM research (and specifically myself as a CAM researcher) can become similarly bound, which created the potential for relationships I have 
demonstrated in this study. Throughout my career, I have been first a practitioner trying to fit into the mainstream (I trained at the same school as these UK therapists), then I became more educated (at University) and frustrated with the mainstream model (like Cyprus practitioner). So I sought to find a way to bring these together (as they do at the Holland centres) but succeeded for the most part in zoning-out much of potential value in order to be acceptable (like the Denmark models). I have therefore proven to be as heterogeneous as CAM therapy!

This is part of the 'messy business' that Freshwater and Rolfe (2001) speak of, which I have chosen to include rather than 'cleanse' from my work. Engaging with this milieu of 'confusion' has been as integral to my research and my findings as the stories of any of the participants I met, and I have endeavoured to include and incorporate what this has tried to teach me in the same way I accepted the Centres that volunteered to participate (as a serendipitous or karmic motion). I feel there are lessons for others too, in observing my struggle to devise, test and apply methods appropriate to the needs I perceived, and in creating appropriate 'space' for this.

Recognising the impact that 'trying to be different' has had on me, and my research, I am no longer surprised that the academics I witnessed in Berlin also struggle to be different and choose instead to present themselves as closer to the mainstream. This is certainly the line of least resistance! This brings me back to serendipity (where I started). If I had not attended that first event at the beginning of my journey, the trajectory I followed since may not have been the one detailed here. An otherwise overlooked connection is that in choosing to present as mainstream those academics have contributed to my trials and tribulations. They have become a part of the context that created such frustration in me as a practitioner and that broke me (through an increasing sense of isolation) as a researcher and a person. To what extent theirs is a representation of also being created or broken by their research context, I must consider but cannot answer here. That is a matter for another study.

This process of becoming a practitioner-researcher has raised many questions for me in regards to authenticity and practice. I have, throughout this study, sought to engage the qualities valued in my clinical practice within my research practice also and this has sometimes felt to broaden the chasm between my research practice and the dominant discourse; to almost parallel the chasm I already felt between my therapy practice and the dominant discourse of medicine. This 'space' has effectively underpinned my entire relationship within this research. Observed first between patients and therapists; patients and therapy; and patients and themselves $\mathrm{I}$ also found this an implicit aspect of my relationships with 
participants; with myself; and with my data. This latter finding is vital as it shows that in therapy and research, you can only ever find what you are willing and able to look for!

Through this study of CAM performances across a range of different environments, the highly variable nature and quality of each context is revealed, exposing both strong themes and rare gems. As a whole production, this data gives a novel and holistic perspective on a number of issues both critical, identified and absent from the preceding knowledge base, which have relevance for both the CAM community (practitioners and patients) and the research community, illuminating a side of daily therapeutic practice, normally unreported.

There is so much more to CAM 'than meets the eye'. These unique modalities offer not only strategies for self empowerment and hope where this may otherwise be lacking, but also provide an alternative choice for people seeking holistic healthcare support. The delivery and experience of these, however, can be actively supported or suppressed by the dramatised discourse in the place where therapy occurs, often in insidious fashion. Put simply, where every sign and symbol says 'focus on the whole person', people invariably can and holistic practice becomes easy. Where the opposite occurs, and focus is directed instead on the illness or complaint, people invariably cannot and holism becomes difficult to enact.

Theatricality, as a methodology, has opened up the space and let me 'into' the various performances of practitioners (and myself): physically, emotionally, psychologically and spiritually. From here, therapy presented a different 'face', one that I felt able to really get to know. This is how I found patients engage and researchers could well do to learn from this. This also suggests that, if CAM practice must be understood in terms of its context, so must CAM research.

I came into this process with the belief that holistic therapies can and should be studied in their whole form, and while at the time I had no idea how this would be achieved, knew this to be my quest. I now know that this is possible, when employing the appropriate tools, as through this study I have developed them. Entry into the theatre demands a special skill, whether audience or actor - the suspension of disbelief. This means leaving your bias, your prejudice and your presumptions at the door as you engage in another 'reality'. Without this, it is impossible to connect with the stories and emotions of the characters; instead you can only see what you want to see. Taking this forward, I am clear that shoehorning holistic and complex practises into reductive and restrictive research models does not allow CAMs to reveal their full potential, and that a holistic model can. Therapy is a holistic event and 
researched holistically, studies can evidence not just the effects of therapy but expose and explore their whole complex performance, which may improve research results.

Linking back to the discussion in (2.3.3.1), it can both be seen and said that paradigm within therapy practice and research study provides the plot that characters enact, within context. Perhaps then, the primary finding of this study is that therapeutic practises observed (and this research practice) share a common thread of managing and mediating an active dichotomy between holism and reductionism that is both unique and local. Reflexive awareness of the influence of this dichotomy, however, on the practises of therapy delivered and the impact of this for the experiences of patients appears itself to be highly variable and heterogeneous sometimes even lacking. Certainly the role played by paradigm presented an additional character within this whole production, not unlike Steven Spielberg's revelation that Dartmoor was a 'third character' in his acclaimed movie War Horse (Groscop, 2012).

From my supervisors who gave the freedom to fly without teaching me first; the other academics, post-graduate students and CAM researchers I encountered who seemed either unaware, scared or (maybe even) too broken by the system they were perpetuating to challenge it; the practitioners who always did their best for their patients within the (perceived) limits of their circumstances; the patients who were often simply glad to be offered something more than conventional/mainstream care; and myself for being so convinced I knew my own mind (when often I did not) I have discovered that research is not only a messy business, but it is neither for the faint-hearted and has the ability to 'undo' a person as much as to 'transform' them. In this sense, research can be as salutogenic as CAM healthcare, where the aim is true development.

One thing is certain, this study has given me the best of times and the worst of times in my life. A most amazing experience, this has afforded me the opportunity to travel to places I would not otherwise have been able to go, become friends with people I would not ordinarily have had the chance to meet, and ultimately grow and develop as a researcher and a person. Plus, spending 3 months living in Holland, Cyprus and Denmark on overseas fieldwork has provided me with many fantastic memories that I will cherish forever as a result. Not without its costs, however, perhaps more in the personal sense than financial one, achieving this meant the structure and dynamics of my whole life became redirected for (more than) a while, and there are long term implications of this that I am yet to fully live out. At times I have also been truly broken by this process (as is evident from the fieldwork chapters) but through the production 
of this final report, I have found salutogenesis too. I have come 'home' to a better place of 'being' - wounded but wiser.

I began this process believing that therapy practice may be captured 'more fully' if viewed holistically as an 'integrated act' of character, plot and set. In doing so, I feel I have revealed aspects of therapy (and their values) previously overlooked, for research to 'look again' at. I believe I have also demonstrated that research is an 'integrated act' of character, plot and set!

This is what my CAM practitioner's perspective of studying CAM practice can teach CAM researchers about researching CAM. 


\section{References}

Ackerman, S.J. and Hilsenroth, M.J. (2003). "A review of therapist characteristics and techniques positively impacting the therapeutic alliance", Clinical Psychology Review, Vol. 23:133

Aickin, M., Bell, I.R., Fønnebø, V., Lewith, G.T., Ritenbaugh, C. and Verhoef, M. (2006). "Methodological Challenges in Whole Systems Research", The Journal of Alternative and Complementary Medicine, Vol. 12 (9):843-850

Anderson, A.R. (2005) "Enacted Metaphor: The Theatricality of the Entrepreneurial Process", International Small Business Journal, Vol.23 (6):587-603

Andrews, G.J. and Boon, H. (2005). "CAM in Canada: places, practice, research", Complementary Therapies in Clinical Practice, Vol. 11:21-27

Andrews, G.J. and Moon, G. (2005a). "Space, Place, and the Evidence Base: Part I - An Introduction to Health Geography", Worldviews on Evidence-Based Nursing, Vol. 2 (2): 55-62

Andrews, G.J. and Moon, G. (2005b). "Space, Place, and the Evidence Base: Part II - Rereading Nursing Environment through Geographical Research", Worldviews on Evidence-Based Nursing, Vol. 2 (3): 142-156

Andrews, G.J., Wiles, J. and Miller, K.L. (2004). "The geography of complementary medicine: perspectives and prospects", Complementary Therapies in Nursing and Midwifery, Vol. 10:175185

Armstrong, D., Kane, M., Reid, D., McBurney, M., Aubrey-Lees, R., Lawson, B. and Buchan, J. (2004). Full Report. The role of hospital design in the recruitment, retention and performance of NHS nurses in England, Belfast: PricewaterhouseCoopers LLP

Aston, E. and Savona, G. (1991). Theatre as a sign-system: a semiotics of text and performance, London: Routledge

Avis, $M$ and Freshwater, D. (2006). "Evidence for practice, epistemology, and critical reflection", Nursing Philosophy, Vol. 7 (4):216-224

Beecher, H.K. (1955). "The Powerful Placebo", Journal of the American Medical Association, Vol. 159:1602-1606 
Bell, P.A., Fisher, J.D., Baum, A. and Greene, T.C. (1996). Environmental Psychology: Fourth Edition, Fort Worth, Texas: Harcourt Brace College Publishers

Bell, I. and Koithan, M. (2006). "Models for the Study of Whole Systems", Integrative Cancer Therapies, Vol. 5 (4):293-307

Biley, F.C. and Freshwater, D. (1998). "Spiritual care and the environment: a new paradigm for nursing?", Complementary Therapies in Nursing and Midwifery, Vol. 4:98-99

Boon, H. and Kachan, N (2008). "Integrative medicine: a tale of two clinics", BMC Complementary and Alternative Medicine, Vol. 8:32

Boon, H., MacPherson, H., Fleishman, S., Grimsgaard, S., Koithan, M., Norheim, J. and Walach, H. (2007). "Evaluating Complex Healthcare Systems: A Critique of Four Approaches", eCAM, Vol. 4 (3):279-285

Burns, E (1972). Theatricality: A study of convention in the theatre and in social life, London: Longman

Busato, A. and Kunzi, B. (2010). "Differences in the quality of interpersonal care in complementary and conventional medicine", BMC Complementary and Alternative Medicine, Vol.10 (63):1-14

Campbell, A. (2000). "Acupuncture, touch, and the placebo response", Complementary Therapies in Medicine, Vol. 8:43-46

Carter, B. (2003). "Methodological issues and complementary therapies: researching intangibles?", Complementary Therapies in Nursing and Midwifery, Vol. 9:133-139

Cassidy, C.M. (1994). "Unravelling the ball of string: Reality, Paradigms and Alternative Medicine", Advances, the Journal of Mind-Body Health, Vol. 10 (1):5-31

Clow, A. (2001). Behavioural conditioning of the immune system. In Peters, D. (Ed.), Understanding the Placebo Effect in Complementary Medicine: Theory, Practice and Research, London: Churchill Livingstone

De Craen, A.J.M., Lampe-Schoenmaeckers, A.J.E.M. and Kleijnen, J. (2001). Non-specific factors in randomised clinical trials: some methodological considerations. In Peters, D. (Ed.), Understanding the Placebo Effect in Complementary Medicine: Theory, Practice and Research, London: Churchill Livingstone 
Dellman, T. and Lushington, K. (2012). "How Natural Therapists enhance positive expectations of patients", Complementary Therapies in Clinical Practice, Vol. 18:99-105

Denzin, N.K. (2003). Performance Ethnography: Critical Pedagogy and the Politics of Culture, Thousand Oaks, California: Sage Publications Inc.

Di Blasi, Z. and Kleijnen, J. (2000). "Consultations at Glasgow Homeopathic Hospital", Report for the Fetzer Institute, USA, No. 1496:1-16

Di Blasi, Z., Harkness, E., Ernst, E., Giorgiou, A. and Kleijnen, J. (2001). "Influence of context effects on health outcomes: a systematic review", The Lancet, Vol. 357:757-762

Dobos, G. (2009). "Integrative Medicine - Medicine of the future or 'Old Wine in new Skins'?", Europe an Journal of Integrative Medicine, Vol. 1:109-115

Duggan, M. and Grainger, R. (1997). Imagination, Identification and Catharsis in Theatre and Therapy, London: Jessica Kingsley Publishers Ltd.

Eardley, S., Bishop, F.L., Prescott, P., Cardini, F., Brinkhaus, B., Santos-Rey, K., Vas, J., von Ammon, K., Hegyi, G., Dragan, S., Uehleke, B., Fønnebø, V. and Lewith, G. (2012). “A Systematic Literature Review of Complementary and Alternative Medicine Prevalence in EU", Forschende Komplementärmedizin, Vol. 19 (suppl. 2):18-28

ECIM (2009). "2 ${ }^{\text {nd }}$ European Congress for Integrative Medicine: The Future of Comprehensive Patient Care", Main conference programme: www.ecim-congress.org

Edensor, T. (2001). "Performing tourism, staging tourism: (Re)producing tourist space and practice", Tourist Studies, Vol. 1 (1):59-81

Elder, C., Aickin, M., Bell, I.R., Fønnebø, V., Lewith, G., Ritenbaugh, C. and Verhoef, M. (2006). "Methodological Challenges in Whole Systems Research", The Journal of Alternative and Complementary Medicine, Vol. 12 (9):843-850

Ernst, E. (2010a). “The public's enthusiasm for complementary and alternative medicine amounts to a critique of mainstream medicine", International Journal of Clinical Practice, Vol. 64 (11):1472-1474

Ernst, E. (2010b). "Are we missing the 'evidence-based boat'?", Focus on Alternative and Complementary Therapies, Vol. 15 (1):1-2 
Ernst, E. (2009). "Research Into Complementary/Alternative Medicine: A Personal Comment", Complementary Health Practice Review, Vol. 14 (1):51-54

Ernst, E. (2001). Towards a scientific understanding of placebo effects. In Peters, D. (Ed.), Understanding the Placebo Effect in Complementary Medicine: Theory, Practice and Research, London: Churchill Livingstone

Ernst, E., Schmidt, K and Wider, B. (2005). "CAM research in Britain: the last 10 years", Complementary Therapies in Clinical Practice, Vol. 11:17-20

EU Commission (2006). "Europeans and Their Languages", Special Eurobarometer 243, http://ec.europa.eu/public_opinion/archives/ebs/ebs_243_sum_en.pdf (access 11/04/12)

Falkenberg, T., Lewith, G., di Sarsini, P.R., von Ammon, K., Santos-Rey, K., Hök, J., Frei-Erb, M., Vas, J., Saller, R. and Uehleke, B. (2012). "Towards a Pan-European Denfinition of Complementary and Alternative Medicine - a Realistic Ambition?", Forschende Komplementärmedizin, Vol. 19, (supp. 2):6-8

Féral, J. (2002). "Theatricality: The Specificity of Theatrical Language", SubStance, Vol. 31 $(2 \& 3): 94-108$

Fischer, H.F., Junne, F., Witt, C., von Ammon, K., Cardini, F., Fönnebö, V., Johannessen, H., Lewith, G., Uehleke, B., Weidenhammer, W. and Brinkhaus, B. (2012). "Key Issues in Clinical and Epidemiological Research in Complementary and Alternative Medicine - a Systematic Literature Review, Forschende Komplementärmedizin, Vol. 19, (supp. 2):51-60

Fischer-Lichte, E. (1995a). "Theatricality: A Key Concept in Theatre and Cultural Studies", Theatre Research International, Vol. 20 (2):85-89

Fischer-Lichte, E. (1995b). "From Theatre to Theatricality -How to Construct Reality", Theatre Research International, Vol. 20 (2):97-105

Fisk, R.P. and Grove, S.J. (1996). "Applications of impression management and the drama metaphor in marketing: and introduction", European Journal of Marketing, Vol. 30 (9):6-12

Fønnebø, V., Grimsgaard, S., Walach, H., Ritenbaugh, C., Norheim, A.J., MachPherson, H., Lewith, G., Launsø, L., Koithan, M., Falkenberg, T., Boon, H. and Aickin, M. (2007). "Researching complementary and alternative treatments - the gatekeepers are not at home", BMC Medical Research Methodology, Vol. 7:7 
Freshwater, D. (1997). "Geopathic stress", Complementary Therapies in Nursing and Midwifery, Vol. 3:160-162

Freshwater, D. (2005). "The poetics of space: researching the concept of spatiality through relationality", Psychodynamic Practice, Vol. 11 (2):177-187

Freshwater, D. and Avis, M. (2004). “Analysing interpretation and reinterpreting analysis: exploring the logic of critical reflection", Nursing Philosophy, Vol. 5 (1):4-11

Freshwater, D. and Rolfe, G. (2001). "Critical reflexivity: A politically and ethically engaged research method for nursing", Nursing Times Research, Vol.6 (1):526-537

Gaboury, I., Toupin April, K. and Verhoef, M. (2012). "A qualitative study on the term CAM: is there a need to reinvent the wheel?", BMC Complementary and Alternative Medicine, Vol. $12: 131$

Gallagher, W. (1993). The Power of Place, New York: HarperCollins Publishers

Gardner, W.L. (1992). "Lessons in Organisational Dramaturgy: The Art of Impression Management", Organisational Dynamics, Vol.21 (1):33-47

Gesler, W.M. (2003). Healing Places, Lanham, MD:Rowman \& Littlefield Publishers, Inc.

Glass, P.G. (2012). “Doing Scene: Identity, Space and the Interactional Accomplishment of Youth Culture", Journal of Contemporary Ethnography, Vol. 41 (6):695-716

Goffman, E. (1959). The Presentation of Self in Everyday Life, London: Penguin

Golden, I. (2012). "Beyond Randomised Controlled Trials: Evidence in Complementary Medicine", Journal of Evidence-Based Complementary \& Alternative Medicine, Vol. 17 (1):7275

Goldstone, L.A., (2000). "Massage as an orthodox medical treatment past and future", Complementary Therapies in Nursing and Midwifery, Vol. 6 (4):169-175

Goodall, Jr, H.L. (2011). Writing the New Ethnography, Oxford: Alta Mira Press

Goodwin, C (1996). "Moving the drama into the factory: the contribution of metaphors to services research", European Journal of Marketing, Vol. 30 (9):13-36

Graham-Hill, S. and Grimes, A.J. (2001). "Dramatism as method: the promise of praxis", Journal of Organisational Change Management, Vol. 14 (3):280-294: 
Greenhalgh, T. (2012). "Why do we always end up here? Evidence-based medicine's conceptual cul-de-sacs and some off-road alternatives", Journal of Primary Health Care, Vol. 4 $(2): 92-97$

Gregson, N. And Rose, G. (2000). "Taking Butler elsewhere: performativities, spatialities and subjectivities", Environment and Planning D: Society and Space, Vol. 18:433-452

Groscop, V. (2012). "How Steven Spielberg found War Horse's third character in Dartmoor", The Observer, Sunday $8^{\text {th }}$ January 2012, Special supplement:6

Hare, A.P. and Blumberg, H.H. (1988). Dramaturgical analysis of social interaction, Westport, CT: Praeger Publishers

Harris, P.E., Cooper, C.L., Relton, C. and Thomas, K.J. (2012). “Prevalence of complementary and alternative medicine (CAM) use by the general population: a systematic review and update", International Journal of Clinical Practice, Vol. 66 (10):924-939

Harris, P., Atkins, R.C. and Alwyn, T. (2010). "Evaluating a complementary therapies clinic: Outcomes and relationships", Complementary Therapies in Clinical Practice, Vol. 16:31-35

Harvey, A. (2001). "A dramaturgical analysis of charismatic leader discourse", Journal of Organisational Change Management", Vol.14 (3):253-265

Hawe, P., Shiell, A. and Riley, T. (2004). "Complex interventions: how 'out of control' can a randomised controlled trial be?", British Medical Journal, Vol. 328:1561-1563

Helman, C.G. (2001). Placebos and nocebos: the cultural construction of belief. In Peters, D. (Ed.), Understanding the Placebo Effect in Complementary Medicine: Theory, Practice and Research, London: Churchill Livingstone

Hoover, J.L. (2012). Spiritual Care in Clinical Nursing Practice: Myth or Reality (unpublished thesis), Leeds, UK: The University of Leeds

House of Lords (2000). "Select Committee on Science and Technology: Sixth Report", http://www.publications.pa rliament.uk/pa/ld199900/Idselect/ldsctech/123/12302. htm

Humphrey, G. (1924). "The Psychology of the Gestalt", The Journal of Educational Psychology, Vol. 15 (7):401-412

Jonas, W.B., Lewith, G. and Walach, H. (2002). Balanced research strategies for complementary and alternative medicine. In Lewith, G., Jonas, W.B. And Walach, H. (Eds.), Clinical Research in 
Complementary Therapies: Principles, Problems and Solutions, Edinburgh: Churchill Livingstone

Jonas, W.B., Beckner, W. and Coulter, I. (2006). “Proposal for an Integrated Evaluation Model for the Study of Whole Systems Health care in Cancer", Integrative Cancer Therapies, Vol. 5 (4):315-319

Jones, J.L. (2002). "Performance Ethnography: The Role of Embodiment in Cultural Authenticity", Theatre Topics, Vol. 12 (1):1-15

Joyce, C.R.B. (1994). "Placebo and complementary medicine”, The Lancet, Vol.344:1279-1281

Kaplan, B.J., Giesbrecht, G., Shannon, S. and McLeod, K. (2011). "Evaluating treatments in health care: the instability of a one-legged stool", BMC Medical Research Methodology, Vol. 11 $(65): 1-7$

Kaptchuk, T.J., Edwards, R.A. and Eisenberg, D.M. (1996). Complementary medicine: efficacy beyond the placebo effect, In Ernst, E. (Ed.), Complementary Medicine: an objective appraisal, Oxford: Butterworth Heinemann

Kaptchuk, T.J., Shaw, J., Kerr, K.E., Conboy, L.A., Kelley, J.M., Csordas, T.J., Lembo, A.J. and Jacobson, E.E. (2009). “"Maybe I Made Up the Whole Thing”: Placebos and Patients' Experiences in a Randomized Controlled Trial", Culture, Medicine and Psychiatry, Vol. 33:382411

Kelner, M. and Wellman, B. (2003). Introduction Complementary and Alternative Medicine: Challenge and Change. In Kelner, M., Wellman, B., Pescosolido, B. and Saks, M. (Eds.), Complementary and Alternative Medicine: Challenge and Change, London: Routledge, Taylor \& Francis

Kienle, G.S. and Kiene, H (2001). A critical reanalysis of the concept, magnitude and existence of placebo effects. In Peters, D. (Ed.), Understanding the Placebo Effect in Complementary Medicine: Theory, Practice and Research, London: Churchill Livingstone

Kipling, R. (1895). "If", http://www.kiplingsociety.co.uk/poems_if.htm (accessed 18/08/2015)

Kleinman, A. (1980). Patients and Healers in the Context of Culture: An Exploration of the Borderland between Anthropology, Medicine and Psychiatry, Berkeley: University of California Press 
Koithan, M., Bell, I.R., Niemeyer, K. and Pincus, D. (2012). “A Complex Systems Science Perspective for Whole Systems of Complementary and Alternative Medicine Research", Forschende Komplementärmedizin, Vol. 19 (suppl. 1):7-14

Kyle, G. and Marks-Maran, D. (2008). "Focus group interviews: How aromatherapists feel about changing their practice through undertaking a randomised controlled trial?", Complementary Therapies in Clinical Practice, Vol. 14:204-211

Launs $\varnothing$, L. (1994). How to kiss a Monster! Designs and paradigms in research on alternative therapy. In Johannessen, H., Launs $\varnothing$, L., Gosvig Olesen, S. And Staugård, F. (Eds.), Studies in Alternative Therapy 1: Contributions from the Nordic Countries, Gylling, Denmark: Odense University Press

Leach, M.J. (2005). "Rapport: A key to treatment success”, Complementary Therapies in Clinical Practice, Vol. 11:262-265

Leather, P., Beale, D., Santos, A., Watts, J. and Lee, L. (2003). “Outcomes of Environmental Appraisal of Different Hospital Waiting Areas", Environment and Behaviour, Vol. 35 (6):842-869

Lees, J. (1997). "An approach to counselling in GP surgeries", Psychodynamic Practice, Vol. 3 $(1): 33-48$

Lees, J. (2013). "Psychotherapy, complementary and alternative medicine and social dysfunction", European Journal of Psychotherapy \& Counselling, Vol. 15 (3):201-213

Lewith, G. (2008). "The Cultural Context of CAM", The Journal of Alternative and Complementary Medicine, Vol.14 (10):1179-1180

Long, A.F. (2007). Executive Summary. Final Report. The Effects and Experience of Shiatsu: A Cross-European Study, Leeds: University of Leeds, School of Healthcare

Long, A.F. and Mackay, H.C. (2003). "The Effects of Shiatsu: Findings from a Two-Country Exploratory Study", The Journal of Alternative and Complementary Medicine, Vol. 9 (4):539547

MacPherson, H., Peters, D. And Zollman, C. (2009). "Closing the evidence gap in integrative medicine" , British Medical Journal, Vol. 339:763-764 
McCall, M., Thorne, S., Ward, A. and Heneghan, C. (2015). "Yoga in adult cancer: an exploratory, qualitative analysis of the patient experience", BMC Complementary and Alternative Medicine, Vol. 15:245

Maple, E. (1992). The Great Age of Quackery, In Saks, M. (Ed.), Alternative Medicine in Britain, Oxford: Clarendon Press

Mason, S., Tovey, P. and Long, A.F. (2002). "Evaluating complementary medicine: methodological challenges of randomised controlled trials", British Medical Journal, Vol. 325:832-834

Mercer, S.W. \& Reilly, D. (2004). "A qualitative study of patient's views on the consultation at the Glasgow Homoeopathic Hospital, an NHS integrative complementary and orthodox medical care unit", Patient Education and Counselling, Vol. 53:13-15

Mitchell, A. and Cormack, M. (1998). The Therapeutic Relationship in Complementary Healthcare, Edinburgh: Churchill Livingstone

Moerman, D.E. (2002). Meaning, medicine and the 'placebo effect', Cambridge: Cambridge University Press

Moustakas, C. (1990). Heuristic Research: design, Methodology and Applications, Newbury Park, CA: Sage Publications Inc.

MRC (2008). "Developing and evaluating complex interventions: new guidance", http://www.mrc.ac.uk/complexinterventionsguidance (accessed 17/05/10)

Mulhall, A. (2001). "Bridging the research-practice gap: breaking new ground in health care", International Journal of Palliative Nursing, Vol. 7 (8):389-394

Mull, J.D. and Mull, D.S. (1983). "Cross-cultural Medicine: A visit with a Curandero", Western Journal of Medicine, Vol. 139:730-736

Myers, W.B. (2010). "The Placebo as Performance: Speaking Across Domains of Healing", Qualitative Health Research, Vol. 20 (9):1295-1303

$\mathrm{NCClH}$ (2015). "What is Complementary and Alternative Medicine?", http://www.nccam.nih.gov/health/integrative-health (access 30/10/15) 
Nissen, N., Schunder-Tatzber, S., Weidenhammer, W. and Johannessen, H. (2012). "What Attitudes and Needs do Citizens in Europe Have in Relation to Complementary and Alternative Medicine", Forschende Komplementärmedizin Vol. 19 (suppl. 2):9-17

Ostermann, T., Blaser, G., Bertram, M., Michalsen, A., Matthiessen, P. and Kraft, K. (2008). "Effects of rhythmic embrocation therapy with Solum oil in chronic pain patients: A Prospective Observational Study", Clinical Journal of Pain, Vol. 24:237-243

Oswick, C., Keenoy, T. and Grant, D. (2001). “Editorial: Dramatising and organising: Acting and being", Journal of Organisational Change Management, Vol. 14 (3):218-224

Paterson, C. and Britten, N (2008). "'The patient's experience of holistic care: insights from acupuncture research", Chronic IIlness, Vol.4:264-277

Paterson, C. and Dieppe, P (2005). "Characteristic and incidental (placebo) effects in complex interventions such as acupuncture", British Medical Journal, Vol. 330:1202-1205

Patsalidis, S. (2011). “(Un)critical (Re)positioning”, Critical Stages: The IATC Webjournal, Issue no. 5, http://www.criticalstages.org (access 22/06/12),

Pedersen, D.M. and Shears, L.M. (1973). "A review of personal space research in the framework of General System theory", Psychological Bulletin, Vol. 80, (5):367-388

Pirie, Z.M., Fox, N.J. and Mathers, N.J. (2012). "Delivering shiatsu in a primary care setting: Benefits and challenges", Complementary Therapies in Clinical Practice, Vol.18:37-42

Raven, B.H. and Rubin, J.Z. (1983). Social Psychology $2^{\text {nd }}$ Edition, New York: John Wiley and Sons Ltd.

Read, G. (2005). "Theatre of Public Space: Architectural experimentation in the Théâtre de I'espace, Paris, 1937", Journal of Architectural Education, Vol. 58 (4):53-62

Ridenour, A. (1998). "Creativity and the Arts in Health Care Settings", Journal of the American Medical Association, Vol. 279 (5):399-400

Ritenbaugh, C., Aickin, M., Bradley, R., Caspi, O., Grimsgaard, S. and Musial, F. (2010). "Whole Systems Research Becomes Real: New Results and Next Steps", The Journal of Alternative and Complementary Medicine, Vol. $16(1): 1-6$ 
Ritenbaugh, C., Verhoef, M., Fleishman, S., Boon, H. and Leis, A. (2003). "Whole Systems Research: A Discipline for Studying Complementary and Alternative Medicine", Alternative Therapies, Vol. 9 (4):32-36

Robinson, J. (2010). Encounters in Child Care, In Ackroyd, A. and O'Toole, J. (Ed.), Performing Research: tensions, triumphs and trade-offs of ethnodrama, London: Institute of Education Press

Robson, C. (2002). Real World Research, Oxford: Blackwell

Rogers, A. (2012). "Geographies of the Performing Arts: Landscapes, Places and Cities", Geography Compass, Vol. 6 (2):60-75

Rossiter, K., Kontos, P., Colantonio, A., Gilbert, J., Gray, J. and Keightley, M. (2008). "Staging data: Theatre as a tool for analysis and knowledge transfer in health research", Social Science \& Medicine, Vol. 66:130-146

Rostock, M., Naumann, J., Guethlin, C., Guenther, L., Bartsch, H.H. and Walach, H. (2011). "Classical homeopathy in the treatment of cancer patients - a prospective observational study of two independent cohorts", BMC Cancer, Vol. 11:19 (p.1-8)

Saldaňa, J. (2011). Ethnotheatre: research from page to stage, Walnut Creek, CA: Left Coast Press Inc

Saunders, T. (2003). "Health hazards and electromagnetic fields", Complementary Therapies in Nursing and Midwifery, Vol. 9:191-197

Shapiro, J. and Hunt, L. (2003). "All the world's a stage: the use of theatrical performance in medical education", Medical Education, Vol. 37:922-927

Shur Bilchik, G. (2002). "A Better Place to Heal”, Health Forum Journal, Vol. 45 (4):10-15

Sloan Devlin, A. and Arneill, A.B. (2003). "Health Care Environments and Patient Outcomes: A Review of the Literature", Environment and Behavior, Vol. 35 (5):665-694

Smith, C.A.M. and Gallo, A.M. (2007). "Applications of Performance Ethnography in Nursing", Qualitative Health Research, Vol. 17 (4):521-528

Smith, J.M., Sullivan, S.J. and Baxter, G.D. (2009). "The culture of massage therapy: Valued elements and the role of comfort, contact, connection and caring", Complementary Therapies in Medicine, Vol. 17:181-189 
Spradley, J.P. (1979). The Ethnographic Interview, Orlando, FL: Holt, Rinehart and Winston Inc Spradley, J.P. (1980). Participant Observation, Orlando, FL: Holt, Rinehart and Winston Inc Steiner, R. (1898). "Science and Criticism", Theatre Pages, Vol. 1 (29). In Bridgemont, P. (Ed.) (2011). Liberation of the Theatre: Rudolph Steiner Theatre Critic Berlin 1897-1900, London: Peter Bridgemont

Steiner, R. (2007). Four Mystery Dramas, Great Barrington, MA:SteinerBooks/Anthroposophic Press

Steiner, R. and Wegman, I. (1925). Fundamentals of Therapy: An Extension of the Art of Healing through Spiritual Knowledge, London: Anthro posophical Publishing co.

Stevenson, H. (2010). "Paradox: A Gestalt Theory of Change for Organizations", Gestalt Review, Vol. $14(2): 111-126$

The Cochrane Library, http://www.thecochranelibrary.com (access 09/11/12)

Thorne, S. (2008). Interpretive Description, Walnut Creek, California: Left Coast Press, Inc.

Toporisic, T. (2011). "Critique and criticism? Can They Survive in a Time of Intertextuality, Intermediality and Corporate Capitalism?", Critical Stages: The IATC Webjournal, Issue no. 5, http://www.criticalstages.org (access 22/06/12)

Turner, R.N., Leach, J. and Robinson, D. (2007). "First impressions in complementary practice: The importance of environment, dress and address to the therapeutic relationship", Complementary Therapies in Clinical Practice, Vol. 13:102-109

Ulrich, R.S. (1979). "Visual landscapes and psychological well-being”, Landscape Research, Vol. 4:17-23

Ulrich, R.S. (1984). "View through a window may influence recovery from surgery", Science, Vol. 224:420-421

Ulrich, R.S. (1986). "Human responses to vegetation and landscape", Landscape and Urban Planning, Vol. 13:29-44

Ulrich, R.S., Simmons, R.F., Losito, B.D., Fiorito, E., Miles, M.A. and Zelson, M. (1991). "Stress recovery during exposure to natural and urban environments", Journal of Environmental Psychology, Vol.11:291-300 
Vallance, A.K. (2006). "Something out of nothing: the placebo effect." Advances in Psychiatric Treatment, Vol.12:287-296

Verhoef, M.J., Lewith, G., Ritenbaugh, C., Thomas, K., Boon, H. and Fønnebø, V. (2004). “Whole Systems Research: moving forward", Focus on Alternative and Complementary Therapies, Vol. $9(2): 87-90$

Verhoef, M.J., Lewith, G., Ritenbaugh, C., Boon, H., Fleishman, S. and Leis, A. (2005). "Complementary and alternative medicine whole systems research: Beyond identification of inadequacies of the RCT", Complementary Therapies in Medicine, Vol. 13:206-212

Verhoef, M.J., Vanderheyden, L.C. and Fønnebø, V. (2006). "A Whole Systems Research Approach to Cancer Care: Why Do We Need It and How Do We Get Started?", Integrative Cancer Therapies, Vol. 5 (4):287-292

Verhoef, M., Koithan, M., Bell, I.R., Ives, J. and Jonas, W. (2012). "Whole Complementary and Alternative Medical Systems and Complexity: Creating Collaborative Relationships", Forschende Komplementärmedizin, Vol. 19 (suppl. 1):3-6

Vickers, A. (1996). Research paradigms in mainstream and complementary medicine, in Ernst, E. (Ed.), Complementary Medicine: an objective appraisal, Oxford: Reed Education and Professional Publishing Ltd

von Ammon, K., Frei-Erb, M., Cardini, F., Daig, U., Dragan, S., Hegyi, G., di Sarsina, P.R., Sörensen, J. and Lewith, G. (2012). "Complementary and Alternative Medicine Provision in Europe - First Results Approaching Reality in an Unclear Field of Practices", Forschende Komplementärmedizin, Vol. 19 (suppl. 2):37-43

Wahlberg, A. (2007). "A quackery with a difference - New medical pluralism and the problem of 'dangerous practitioners' in the United Kingdom", Social Science \& Medicine, Vol. 65:23072316

Walach, H. (2012). "CAMbrella - Complementary Medicine Research in Europe", Forschende Komplementärmedizin Vol. 19 (suppl. 2):1-2

Walach, H., Falkenberg, T., Fønnebø, V., Lewith, G. and Jonas, W.B. (2006). “Circular instead of hierarchical: methodological principles for the evaluation of complex interventions", BMC Medical Research Methodology, Vol. 6 (29):1-9 
Walach, H. and Pincus, D. (2012). "Kissing Descartes Good Bye", Forschende Komplementärmedizin, Vol. 19 (suppl. 1):1-2

Walker, R. and Monin, N. (2001). "The purpose of the picnic: using Burke's dramatistic pentad to analyse a company event", Journal of Organisational Change Management, Vol. 14 (3):266279

Wardle, J.L. (2008). "Involve complementary medicine practitioners in research", British Medical Journal, Vol. 337

White, A. (2000). "Integration...Beyond Placebo?”, Complementary Therapies in Medicine, Vol. $8: 225$

WHO (2000). "General Guidelines for Metho dologies on Research and Evaluation of Traditional Medicine", http://whqlibdoc.who.int/hq/2000/WHO_EDM_TRM_2000.1.pdf (access 01/09/13)

Wieland, L.S., Manheimer, E. and Berman, B.M. (2011). “Development and classification of an operational definition of complementary and alternative medicine for the Cochrane collaboration", Alternative Therapies in Health and Medicine, Vol.17 (2):50-59

Wiesener, S., Falkenberg, T., Hegyi, G., Hök, J., di Sarsini, P.R. and Fønnebø, V. (2012). "Legal Status and Regulation of Complementary and Alternative Medicine in Europe", Forschende Komplementärmedizin Vol. 19 (suppl. 2):29-36

Wilkinson, S., Barnes, K and Storey, L. (2008). “Massage for symptom relief in patients with cancer: systematic review", Journal of Advanced Nursing, Vol. 63 (5):430-439

Wood, T. (2002). "Spectacular metaphors: From theatre to cinema", Journal of Organisational Change Management, Vol. 15 (1):11-20

Zollman, C. and MacPherson, H. (2009). "Challenges in interpreting and applying the evidence for CAM and IM", Journal of Holistic Healthcare, Vol. 6 (1):16-21 


\section{Appendix 1: $\quad$ Practitioner Interview Schedule}

1. I'd like to hear at little about your personal journey to becoming a practitioner (including all training you have had) and your post qualifying work history up to joining this clinic?

2. Can you tell me about the work that you currently (personally) do here, both on a therapeutic level and an organisational level?

3. How appropriate or ideal do you regard this setting for your work, and why?

4. Can you compare for me your experiences of working in this environment to other places you may have worked in?

5. Do you feel that the way you work is influenced by any wider social factors e.g. public expectation, legal restrictions/requirements, economics etc?

6. Do particular aspects of this setting influence the way you deliver your treatment - and can you give examples of this?

7. Have you modified this space to better suit your work, and in what way?

8. What do you personally like or dislike about the treatment spaces, communal facilities and general design of the clinic?

9. What, if any, direct effects does the environment you provide here have on you?

10. What, if any, direct effects do you think the environment you provide here has on your patients?

11. Is the issue of environment (clinic and wider social) something that you have ever reflected on before, and for what purpose?

12. Do you regard either yourself or the setting of therapy to be a part of your work or apart from your work, and why? 


\section{Appendix 2: $\quad$ Patient Interview Schedule}

1. Can you tell me about the therapy/treatment you receive here?

2. I'd like to hear your views about this particular clinic; for example things that you like or don't like?

3. How suitable an environment do you feel this clinic is for the kind of therapy that you receive here?

4. Are there any issues for you around accessibility, transport and cost?

5. Can you compare for me your experiences here to other clinics you may have visited?

6. Can you identify any aspects of the treatment room specifically, or the setting generally, that have a direct effect on you, your experience of treatment or your responses to therapy? 


\section{Appendix 3: $\quad$ Interview data extraction sheets}

Interview location:

Name of therapist:

Name of participant:

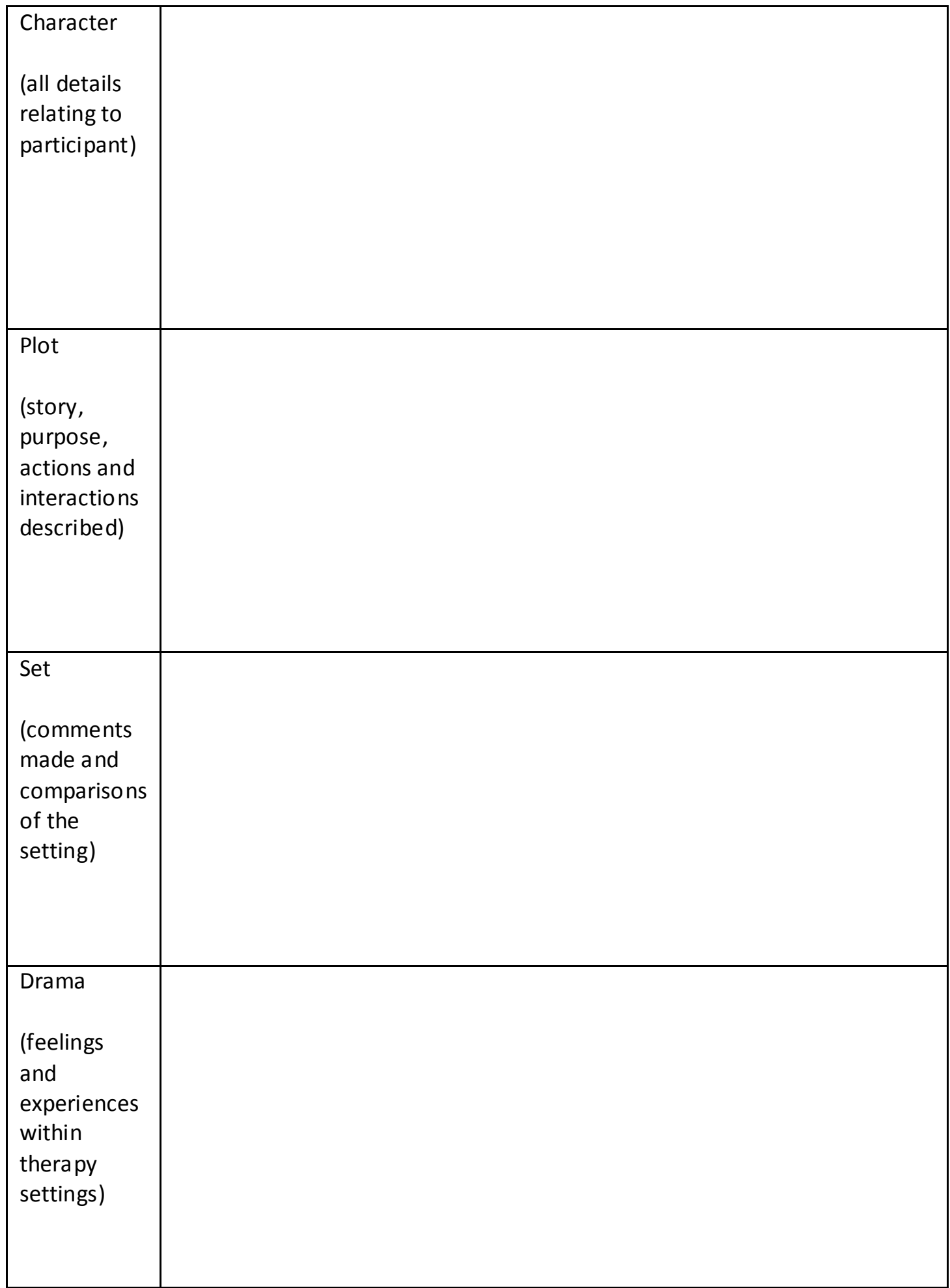




\title{
Appendix 4
}

\section{Comparing complementary health centres in the UK and Europe that use a combination of therapies}

\author{
Sarah Croke*, School of Healthcare, University of Leeds, Leeds, LS2 9UT \\ Supervisors: Dr. John Lees, Prof. Dawn Freshwater *email: hcsc@leeds.ac.uk \\ *tel: 01133433291
}

\begin{abstract}
Study Information Sheet (for Clinics)
(please read in conjunction with participant information sheet(s) and consent form(s))
\end{abstract}

\begin{abstract}
Why is this study being conducted?
Complementary and Alternative Medicine (CAM) treatments do not occur in isolation from practitioners, and practitioners do not practice in isolation from their environments, yet research trial designs often seek to control or eliminate personal variations in practice. In order to clarify the value of contextual factors in complementary therapy, this study aims to explore complementary healthcare settings in the UK and Europe, and the effects of these on practitioners' and patients' experiences of treatment.
\end{abstract}

\section{What does the researcher want to find out?}

This study asks "Is contextual staging influential in the practice and experience of CAM, and how is this manifest?"

\section{How will this be done?}

The Researcher will spend a maximum of 1 month in 4 Complementary Health Centres from different countries within Europe and be present during the working day (frequency and duration to be agreed between the researcher and Centre management) to observe the presentation and use of the clinical environment (both internally and externally), detailing how actions occur within the space. Photographs of the external and internal environments will also be taken (without any persons present) and some actual treatment sessions may be observed. Following this observation period, practitioners and patients will be invited to explore the observational data in one-to-one interviews. Practitioner interviews will be designed to explore the factors that underpin the observed actions and use of therapeutic spaces, and identify whether purposeful manipulation of the space (where this occurs) is intended to affect the patient directly or considered an integral practice/practitioner enhancing intervention. Patient interviews will aim to elicit patient experiences of treatment within their unique context and their views regarding non-specific effects on their own treatment experiences and responses to therapy. These interviews will be audio taped, and all participants will be offered an informal debriefing to allow any problems or issues arising from the exchanges to be raised. For clinic managers this will be at agreed time-points during the fieldwork, and for practitioners/patients this will be immediately after their interviews. Participating practitioners and patients will not be permitted to view each others responses, and under no circumstances will participants' names or identifying characteristics be included in any publications.

\section{What would the clinic have to do?}

Partner with the Researcher in executing the various study activities. This may include agreeing an observation timetable, facilitating access to potential participants 
(practitioners and patients) and where possible providing a suitable space for participant interviews to be conducted.

\section{How will this information help?}

This study will answer many important questions about the value and role of contextual factors in complementary therapy with the aim of informing future research trial design. 


\title{
Appendix 5
}

\section{Comparing complementary health centres in the UK and Europe that use a combination of therapies}

\author{
Sarah Croke*, School of Healthcare, University of Leeds, Leeds, LS2 9JT \\ Supervisors: Dr. John Lees, Prof. Dawn Freshwater *email: hcsc@leeds.ac.uk \\ *tel: 0113-343 3291 \\ Study Information Sheet (for Patients) \\ (a participant information sheet and consent form will be provided upon request)
}

\section{Why is this study being conducted?}

The clinic where you attend for therapy has agreed to participate in a research study conducted by the University of Leeds. The overall aim of the study is to clarify the effects of complementary healthcare settings on practitioners' and patients' experiences of treatment. The study is being conducted in different settings in the UK and Europe. The Researchers believe that Complementary and Alternative Medicine (CAM) treatments do not occur in isolation from practitioners, and practitioners do not practice in isolation from their environments, and wish to explore any personal variations in practice to benefit future research trial designs.

\section{What does the researcher want to find out?}

This study aims to discover if the individual nature of healthcare settings influences treatment delivery and practitioners' / patients' experiences of CAM therapy.

\section{How will this be done?}

The study will run for a maximum of 1 month in 4 Complementary Health Centres from different countries within Europe. This Centre is scheduled to participate between (insert relevant dates). The researcher will be present during the working day (frequency and duration to be agreed between the researcher and Centre management) to observe the presentation and use of the internal (communal and therapeutic) and external clinic environment detailing how the settings are constructed and the impact of these on actions that occur within the spaces. They will not observe your actual treatment sessions unless you consent to this. Your own clinical treatment will be unaffected. Following this observation period, the Researcher would like to interview you about your experience of treatment within this setting.

\section{What would I have to do?}

If you would like to participate in an interview and/or permit an actual treatment observation, then please either contact Sarah Croke (details above*) or complete and return the attached slip to the clinic $^{\star \star}$. You will then be provided with a more detailed participant information sheet, with consent only being taken on the day of your participation. Interviews will last no more than $30 \mathrm{mins}$ and will be audio taped. All participants will be offered an informal debriefing to allow any problems or issues arising 
from the interviews and/or observations to be raised. For patients this will be immediately after your interview/observation.

Participating practitioners and patients will not be permitted to view each others responses, and under no circumstances will participants' names or identifying characteristics be included in any publications.

\section{How will this information help?}

This study will answer many important questions about the value and role of individual healthcare settings in complementary therapy with the aim of informing future research trial design.

${ }^{\star *}$ Please deposit this slip in the return box located in the Clinic Reception area where it will be collected by the Researcher.

I am interested in participating in the above study:

NAME:

You may contact me on TEL: 


\title{
Appendix 6
}

\section{"Comparing complementary health centres in the UK and Europe that use a combination of therapies"}

\author{
Sarah Croke*, School of Healthcare, University of Leeds, Leeds, LS2 9UT \\ Supervisors: Dr. John Lees, Prof. Dawn Freshwater *email: hcsc@leeds.ac.uk \\ *tel: 01133433291

\section{Patient Participant Information Sheet}

We would like you to take part in the above named study but before you decide, please read the following information.

\section{What is the purpose of this study?}

This study aims to discover the influence of complementary healthcare settings on the practice and experience of complementary therapy in the UK and Europe. By examining individual healthcare settings, personal variations in practice may be explored, popular understanding of complementary therapies can be enhanced and future research trial designs improved.

\section{Who is doing the study?}

The study is being conducted Sarah Croke, a practising therapist and PhD Student in the School of Healthcare at the University of Leeds. Her supervisors are Dr. John Lees and Prof. Dawn Freshwater.

\section{Why have I been asked to participate?}

The clinic you attend has agreed to participate in the study. As you are due to attend for therapy during the study period, the Researcher would like permission to observe you in this healthcare setting and/or hear your views regarding the effects of this healthcare setting on your experience of therapy.

\section{What will be involved if I take part in this study?}

You may choose to participate either in the observation phase of the study, the interview phase or both phases.

Observation Phase: The Researcher wants to observe how clinical/therapeutic settings are arranged and used in a real-world situation, including the décor, any furniture, lighting, objects, attire, personal styling and music (if applicable). They will be present during your usual treatment session to note these practicalities, but will not interfere with your usual care. Should you become distressed at any time, you may request to suspend the observation period.

Interview Phase: The Researcher would like to interview you to explore if/how any of the factors observed in the clinical/therapeutic setting you attend influence your experience of complementary therapy and if/whether/how you feel these relate to your treatment experiences and responses to therapy. This may be done in the clinical setting or at an 
appropriate alternative place and will be audio taped. Should you become distressed at any time, you may request to suspend the interview.

The researcher will be on-site for up to 1 month to gather this information. They will not be evaluating your practitioners' competence, or asking controversial or sensitive questions.

\section{What are the advantages and disadvantages of taking part?}

Advantages: Opportunity to participate in an international research project that aims to influence future generation of a relevant and robust evidence-base for CAM therapy; contribute to the enhancement of popular understanding of complementary therapies.

Disadvantages: Intrusion into personal therapeutic care; time investment.

\section{Can I withdraw from the study at any time?}

You can withdraw from the study at any time without needing to give a reason.

If you are withdrawing from the Observation Phase, the Researcher will leave the room immediately.

If you are withdrawing from the Interview Phase, the researcher will stop the interview and switch off the audio equipment.

Any data already collected will be retained in the study.

\section{Will the information I give be kept confidential?}

All information obtained will be treated confidentially. Participating practitioners and patients will not be permitted to view each others responses. Field notes and interview transcripts will only be available to members of the supervisory team, while participants' personal details and the coding system used to identify participants within the observation/interview data will only be available to the Researcher. All 'hard copy' study data and personal details will be stored in locked filing cabinets in separate locations within the School of Healthcare at the University of Leeds. Electronic data will be stored securely on the University server. The data obtained from this study will only be used for the purpose of this study.

All data handling procedures are in accordance with the Data Protection Act 1998.

\section{What will happen to the results of the study?}

It is anticipated that the findings from this study will have relevance for researchers, practitioners, patients, physicians and funding bodies and will therefore be disseminated widely in high impact complementary therapy and other social science and/or health related research journals, by presentation at both national and international research conferences, and other media outlets. Beyond this, and given the innovative nature of the study, it may result in a book or book chapters. While excerpts from observational notes and interview transcripts may appear in both the final research report and other publications, under no circumstances will your name or any identifying characteristics be included.

\section{Who has reviewed this study?}

Ethical approval for this study has been granted the University of Leeds, School of Healthcare Research Ethics Committee. This study has also been approved by the participating Centres' ethics committee (where applicable). 
If you agree to take part, would like more information or have any questions or concerns about the study please contact:

Sarah Croke, lead researcher (contact details at the top of this information sheet).

Thank you for taking the time to read this information sheet. 


\title{
Appendix 7
}

\section{Comparing complementary health centres in the UK and Europe that use a combination of therapies}

\author{
Sarah Croke*, School of Healthcare, University of Leeds, Leeds, LS2 9UT \\ Supervisors: Dr. John Lees, Prof. Dawn Freshwater *email: hcsc@leeds.ac.uk \\ *tel: 01133433291
}

\section{Practitioner Participant Information Sheet}

We would like you to take part in the above named study but before you decide, please read the following information.

\section{What is the purpose of this study?}

This study aims to clarify the role of contextual factors in the practice and experience of complementary therapy in the UK and Europe. By examining individual healthcare settings, personal variations in practice may be explored, popular understanding of complementary therapies can be enhanced and future research trial designs improved.

\section{Who is doing the study?}

The study is being conducted Sarah Croke, a practising therapist and PhD Student in the School of Healthcare at the University of Leeds. Her supervisors are Dr. John Lees and Prof. Dawn Freshwater.

\section{Why have I been asked to participate?}

The clinic where you work has shown interest in participating in the study. Therefore, the Researcher would like permission to observe how you present and use your therapeutic space in this healthcare setting and/or hear your views regarding the effects of this healthcare setting on your experience of delivering therapy.

\section{What will be involved if I take part in this study?}

You may choose to participate in either part of the observation phase of the study, the interview phase or both phases.

Observation Phase: The Researcher wants to observe how your clinical/therapeutic space is arranged and used in real-world situations, including the décor, any furniture, lighting, objects, attire, personal styling and music (if applicable). There are 2 separate parts to this phase.

a) Observe and Photograph the Clinical Space (vacant). This will consist of admitting the Researcher to document the presentation of your clinical/therapeutic setting.

b) A Treatment Observation. The Researcher will be present during a treatment session to note the impact of the clinical presentation on your practice, but will not interfere with your usual care. If you practice multiple therapies, it would be useful to explore any variations in your use of the space/setting. 
Should you become distressed at any time, you may request to suspend the observation period.

Interview Phase: The Researcher would like to interview you to explore if/how any of the factors observed in the setting influence your experience or delivery of complementary therapy and if/whether/how you feel these relate to patient responses to therapy. This may be done in your clinical setting or at an appropriate alternative place and will be audio taped. Should you become distressed at any time, you may request to suspend the interview.

The researcher will be on-site for up to 1 month to gather this information. They will not be evaluating your competence, or asking controversial or sensitive questions. In the unlikely event that any issue of concern is documented (harmful/ethical etc.) this may be raised with you and/or your professional association.

\section{What are the advantages and disadvantages of taking part?}

Advantages: Opportunity to participate in an international research project that aims to influence future generation of a relevant and robust evidence-base for CAM therapy; potentially acquire new knowledge and skills for critiquing, reflecting and improving on your own clinical practice; contribute to the enhancement of popular understanding of complementary therapies; develop your professional reputation (a research active practitioner) and submit details of participation to your professional body for CPD accreditation (where applicable).

Disadvantages: Intrusion into personal therapeutic space; time investment.

\section{Can I withdraw from the study at any time?}

You can withdraw from the study at any time without needing to give a reason.

If you are withdrawing from the Observation Phase, the Researcher will leave the room immediately.

If you are withdrawing from the Interview Phase, the researcher will stop the interview and switch off the audio equipment.

Any data already collected will be retained in the study.

\section{Will the information I give be kept confidential?}

All information obtained will be treated confidentially. Participating practitioners and patients will not be permitted to view each others responses. Field notes and interview transcripts will only be available to members of the supervisory team, while participants' personal details and the coding system used to identify participants within the observation/interview data will only be available to the Researcher. All 'hard copy' study data and personal details will be stored in locked filing cabinets in separate locations within the School of Healthcare at the University of Leeds. Electronic data will be stored securely on the University server. The data obtained from this study will only be used for the purpose of this study.

All data handling procedures are in accordance with the Data Protection Act 1998.

\section{What will happen to the results of the study?}

It is anticipated that the findings from this study will have relevance for researchers, practitioners, patients, physicians and funding bodies and will therefore be disseminated 
widely in high impact complementary therapy and other social science and/or health related research journals, by presentation at both national and international research conferences, and other media outlets. Beyond this, and given the innovative nature of the study, it may result in a book or book chapters. While excerpts from observational notes and interview transcripts may appear in both the final research report and other publications, under no circumstances will your name or any identifying characteristics be included.

\section{Who has reviewed this study?}

Ethical approval for this study has been granted the University of Leeds, School of Healthcare Research Ethics Committee. This study has also been approved by the participating Centres' ethics committee (where applicable).

If you agree to take part, would like more information or have any questions or concerns about the study please contact:

Sarah Croke, lead researcher (contact details at the top of this information sheet) Thank you for taking the time to read this information sheet. 


\section{Appendix 8}

Practitioner Consent Form

\section{"Comparing complementary health centres in the UK and Europe that use a combination of therapies"}

Sarah Croke*, School of Healthcare, University of Leeds, Leeds, LS2 9UT Supervisors: Dr. John Lees, Prof. Dawn Freshwater *email: hcsc@leeds.ac.uk *tel: 01133433291

The participant should complete the whole of this sheet himself/herself

\begin{tabular}{|c|c|}
\hline Practitioner Participant Consent Form & $\begin{array}{l}\text { Please confirm the } \\
\text { statements by } \\
\text { putting your } \\
\text { initials in the box } \\
\text { below }\end{array}$ \\
\hline I have read and understood the participant information sheet & \\
\hline $\begin{array}{l}\text { I have had the opportunity to ask questions and discuss this } \\
\text { study }\end{array}$ & \\
\hline I have received satisfactory answers to all of my questions & \\
\hline I have received enough information about the study & \\
\hline $\begin{array}{l}\text { I understand that I am free to withdraw from the study:- } \\
1 \text { At any time } \\
2 \text { Without having to give a reason for withdrawing } \\
3 \text { That any data already collected will be retained in the } \\
\text { study }\end{array}$ & \\
\hline $\begin{array}{l}\text { I understand that any information I provide, including personal } \\
\text { details, will be confidential, stored securely and only accessed } \\
\text { by those carrying out the study. }\end{array}$ & \\
\hline $\begin{array}{l}\text { I understand that any information I give may be included in } \\
\text { published documents but my identity will be protected by the } \\
\text { use of pseudonyms }\end{array}$ & \\
\hline \multicolumn{2}{|c|}{ I agree to take part in this study (Observe and Photograph Clinical Space) } \\
\hline \multicolumn{2}{|l|}{ I agree to take part in this study (Treatment Observation) } \\
\hline I agree to take part in this study (Audio Taped Interview) & \\
\hline
\end{tabular}




\begin{tabular}{|l|}
\hline Participant Signature $\ldots \ldots \ldots \ldots \ldots \ldots \ldots \ldots \ldots \ldots$ \\
\hline Name of Participant \\
\hline Researcher Signature $\ldots \ldots \ldots \ldots \ldots \ldots \ldots \ldots \ldots \ldots \ldots \ldots$ \\
\hline Name of Researcher \\
\hline
\end{tabular}

Thank you for agreeing to take part in this study. 


\section{Appendix 9}

\section{Patient Consent Form}

\section{"Comparing complementary health centres in the UK and Europe that use a combination of therapies"}

Sarah Croke*, School of Healthcare, University of Leeds, Leeds, LS2 9UT Supervisors: Dr. John Lees, Prof. Dawn Freshwater *email: hcsc@leeds.ac.uk *tel: 01133433291

The participant should complete the whole of this sheet himself/herself

\begin{tabular}{|l|l|}
\hline \multicolumn{1}{|c|}{ Patient Participant Consent Form } & $\begin{array}{l}\text { Please confirm } \\
\text { the statements by } \\
\text { putting your } \\
\text { initials in the box } \\
\text { below }\end{array}$ \\
\hline I have read and understood the participant information sheet & \\
\hline $\begin{array}{l}\text { I have had the opportunity to ask questions and discuss this } \\
\text { study }\end{array}$ & \\
\hline $\begin{array}{l}\text { I have received satisfactory answers to all of my questions } \\
\text { I have received enough information about the study }\end{array}$ & \\
\hline $\begin{array}{l}\text { I understand that I am free to withdraw from the study:- } \\
\text { Without having to give a reason for withdrawing } \\
\text { study any data already collected will be retained in the }\end{array}$ & \\
\hline $\begin{array}{l}\text { I understand that any information I provide, including } \\
\text { personal details, will be confidential, stored securely and } \\
\text { only accessed by those carrying out the study. }\end{array}$ & \\
\hline $\begin{array}{l}\text { I understand that any information I give may be included in } \\
\text { published documents but my identity will be protected by the } \\
\text { use of pseudonyms }\end{array}$ & \\
\hline $\begin{array}{l}\text { I agree to take part in this study (Treatment Observation) } \\
\text { I agreed to take part in this study (Audio Taped Interview) }\end{array}$ & \\
\hline $\begin{array}{l}\text { Name of Participant } \\
\text { Datipant Signature ............................. }\end{array}$ & \\
\hline
\end{tabular}


Researcher Signature

Date

Name of Researcher

Thank you for agreeing to take part in this study 